\title{
Construction of 1,2,3-benzodiazaborole by electrophilic borylation of azobenzene and nucleophilic dialkylative cyclization
}

\author{
Masanori Shigeno, ${ }^{* 1}$ Masaya Imamatsu, ${ }^{1}$ Yusuke Kai, ${ }^{1}$ Moe Kiriyama, ${ }^{1}$ Shintaro Ishida,${ }^{2}$ Kanako Nozawa- \\ Kumada,${ }^{1}$ and Yoshinori Kondo ${ }^{1}$ \\ ${ }^{1}$ Department of Biophysical Chemistry, Graduate School of Pharmaceutical Science, \\ Tohoku University, Aoba, Sendai, 980-8578, Japan \\ ${ }^{2}$ Department of Chemistry, Graduate School of Science, \\ Tohoku University, Sendai 980-8578, Japan \\ *E-mail: masanori.shigeno.e5@tohoku.ac.jp
}

Supporting Information

Table of Contents

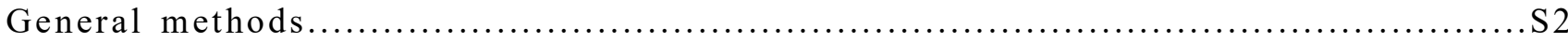

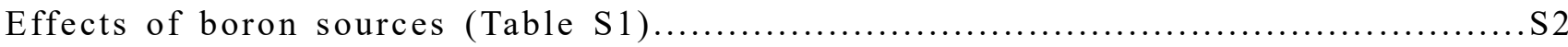

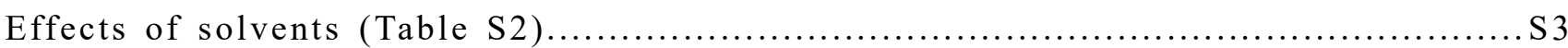

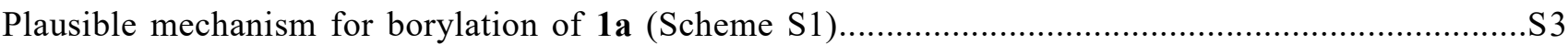

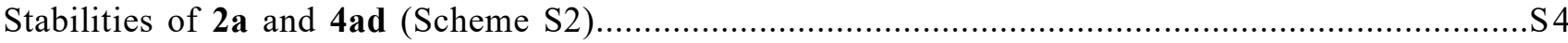

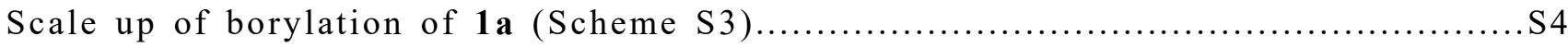

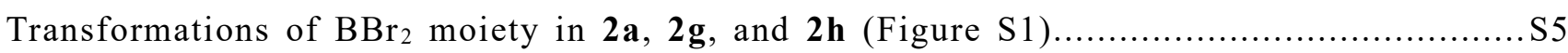

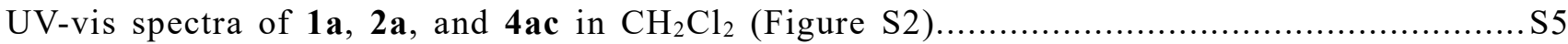

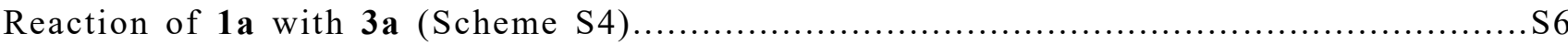

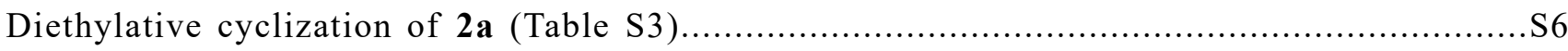

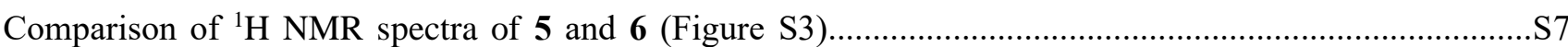

Experimental procedures and spectra data for obtained products............................................S8-S30

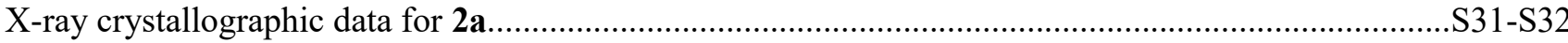

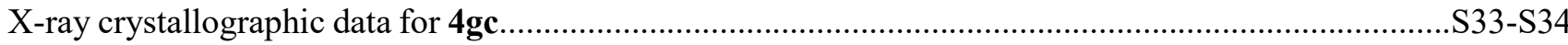

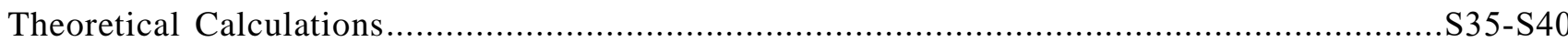

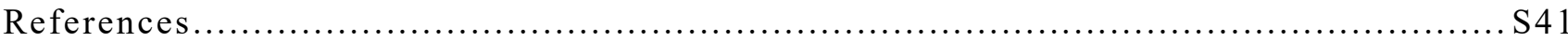

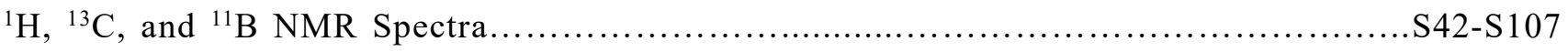


General methods. All reactions were carried out under $\mathrm{N}_{2}$ or Ar atmosphere. Flash column chromatography was performed with Kanto silica gel $60 \mathrm{~N}$ (spherical, neutral, 70-230 $\mu \mathrm{m}$ ). Melting points (Mp) were determined with a Yazawa micro melting point apparatus without correction. Infrared (IR) data were recorded on SensIR ATR (Attenuated Total Reflectance) FT-IR, and absorbance frequencies are reported in reciprocal centimeters $\left(\mathrm{cm}^{-1}\right)$. NMR data were recorded on a JEOL AL400 spectrometer (395.75 MHz for ${ }^{1} \mathrm{H}, 99.50 \mathrm{MHz}$ for ${ }^{13} \mathrm{C}$ ) or a JEOL ECA600 spectrometer (597.17 MHz for ${ }^{1} \mathrm{H}, 150.907 \mathrm{MHz}$ for ${ }^{13} \mathrm{C}, 192.56 \mathrm{MHz}$ for ${ }^{11} \mathrm{~B}$ ). Chemical shifts are expressed in $\delta$ (parts per million, ppm) values, and coupling constants are expressed in herts (Hz). ${ }^{1} \mathrm{H}$ NMR spectra were referenced to tetramethylsilane as an internal standard or to a solvent signal $\left(\mathrm{CDCl}_{3}: 7.26 \mathrm{ppm}\right.$; $\mathrm{CD}_{2} \mathrm{Cl}_{2}$ : $5.32 \mathrm{ppm} ; \mathrm{C}_{6} \mathrm{D}_{6}: 7.16 \mathrm{ppm}$; DMSO- $\left.d_{6}: 2.50 \mathrm{ppm}\right) .{ }^{13} \mathrm{C}$ NMR spectra were referenced to a solvent signal $\left(\mathrm{CDCl}_{3}: 77.0 \mathrm{ppm} ; \mathrm{CD}_{2} \mathrm{Cl}_{2}: 53.8 \mathrm{ppm} ; \mathrm{C}_{6} \mathrm{D}_{6}: 128.0 \mathrm{ppm}\right.$; DMSO-d6: $\left.39.5 \mathrm{ppm}\right) .{ }^{11} \mathrm{~B}$ $\mathrm{NMR}$ spectra were referenced to $\mathrm{BF}_{3} \cdot \mathrm{OEt}_{2}(0.00 \mathrm{ppm})$ as an external standard. Low and high resolution mass spectra (LRMS and HRMS) were obtained from Mass Spectrometry Resource, Graduate School of Pharmaceutical Sciences, Tohoku University, on a JEOL JMS-DX 303 and JMS-700/JMS-T 100 GC spectrometer, respectively. UV-vis spectra were measured on a JASCO J-720 spectropolarimeter. X-ray diffraction data were recorded on a Rigaku RAXIS-RAPID imaging plate diffractometer with graphite-monochromated Mo-Ka radiation.

Table S1. Effects of boron sources ${ }^{a}$

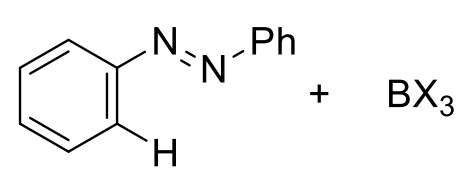

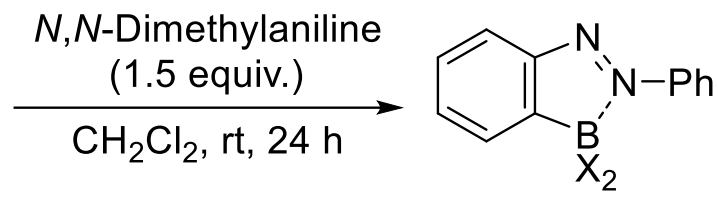

$1 \mathrm{a}$

(3.0 equiv.)

$2 a$

\begin{tabular}{|c|c|c|}
\hline Entry & Boron source & Yield of 2a $(\%)^{b}$ \\
\hline 1 & $\mathrm{BF}_{3} \cdot \mathrm{Et}_{2} \mathrm{O}$ & 0 \\
\hline 2 & $\mathrm{BCl}_{3}$ & 1 \\
\hline 3 & $\mathrm{BBr}_{3}$ & $(98)^{c}$ \\
\hline 4 & $\mathrm{BBr}_{3}(2.0$ equiv. & $(96)^{c}$ \\
\hline
\end{tabular}

${ }^{a} 1 \mathrm{a}(0.20 \mathrm{mmol}), \mathrm{BX}_{3}\left\{\mathrm{BF}_{3} \cdot \mathrm{Et}_{2} \mathrm{O}(0.60 \mathrm{mmol}), \mathrm{CH}_{2} \mathrm{Cl}_{2}(0.60 \mathrm{~mL})\right.$ (entry 1$) ; \mathrm{BCl}_{3}(1.0 \mathrm{M}$ in heptane, $0.60 \mathrm{~mL}, 0.60 \mathrm{mmol}), \mathrm{CH}_{2} \mathrm{Cl}_{2}(0.60 \mathrm{~mL})$ (entry 2); $\mathrm{BBr}_{3}\left(1.0 \mathrm{M}\right.$ in $\left.\mathrm{CH}_{2} \mathrm{Cl}_{2}, 0.60 \mathrm{~mL}, 0.60 \mathrm{mmol}\right)$ (entry 3); $\mathrm{BBr}_{3}\left(1.0 \mathrm{M}\right.$ in $\left.\mathrm{CH}_{2} \mathrm{Cl}_{2}, 0.40 \mathrm{~mL}, 0.40 \mathrm{mmol}\right), \mathrm{CH}_{2} \mathrm{Cl}_{2}(0.20 \mathrm{~mL})$ (entry 4)\}, N,N- 
dimethylaniline $(0.30 \mathrm{mmol})$, rt, $24 \mathrm{~h} .{ }^{b}$ Yields were determined by ${ }^{1} \mathrm{H}$ NMR spectroscopy using 1,1,2trichloroethane as an internal standard. ${ }^{c}$ Yield in parentheses denotes the isolated yield.

Table S2. Effects of solvents ${ }^{a}$

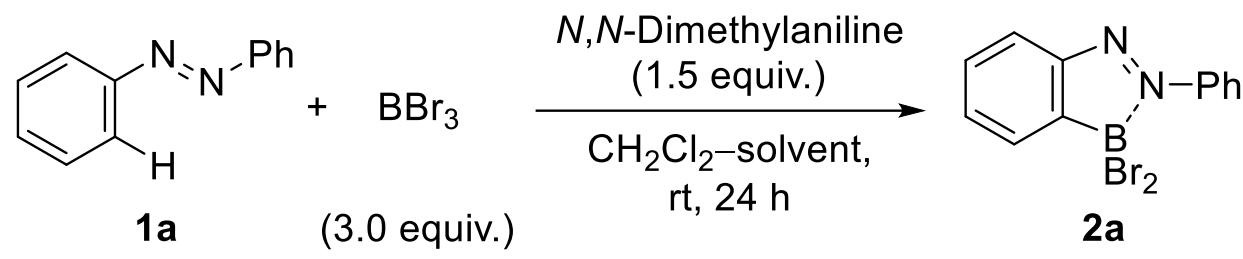

\begin{tabular}{|c|c|c|}
\hline Entry & Additional solvent & ${\text { Yield of } \mathbf{2 a}(\%)^{b}}^{b}$ \\
\hline 1 & - & $(98)^{c}$ \\
\hline 2 & $\mathrm{CH}_{2} \mathrm{Cl}_{2}$ & $(93)^{c}$ \\
\hline 3 & $\mathrm{THF}$ & 0 \\
\hline 4 & $\mathrm{Et}_{2} \mathrm{O}$ & 0 \\
\hline 5 & $\mathrm{Cyclohexane}^{c}$ & $(87)^{c}$ \\
\hline 6 & Benzene & $(91)^{c}$ \\
\hline
\end{tabular}

${ }^{a} 1 \mathrm{a}(0.20 \mathrm{mmol}), \mathrm{BBr}_{3}\left(1.0 \mathrm{M}\right.$ in $\left.\mathrm{CH}_{2} \mathrm{Cl}_{2}, 0.60 \mathrm{~mL}, 0.60 \mathrm{mmol}\right), N, N$-dimethylaniline $(0.30 \mathrm{mmol})$, additional solvent $(0.60 \mathrm{~mL})$, rt, $24 \mathrm{~h} .{ }^{b}$ Yields were determined by ${ }^{1} \mathrm{H}$ NMR spectroscopy using 1,1,2trichloroethane as an internal standard. ${ }^{c}$ Yield in parentheses denotes the isolated yield.

Scheme S1. Plausible mechanism for borylation of 1a

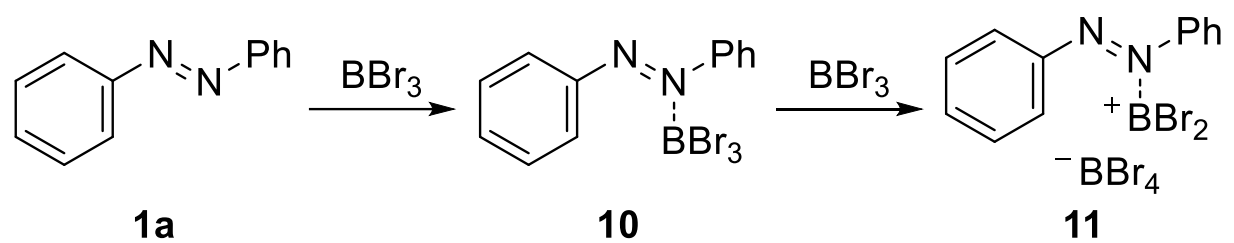

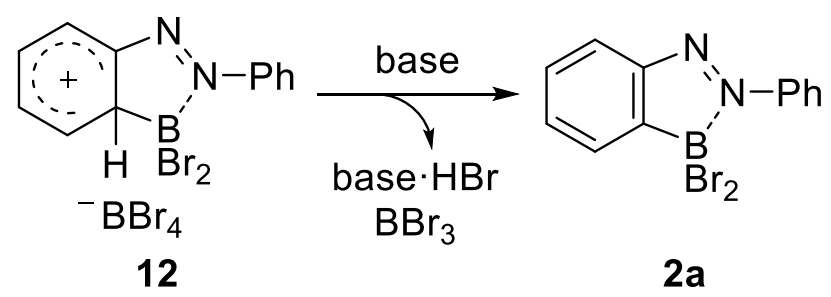


Scheme S2. Stabilities of $2 \mathbf{a}$ and $4 \mathbf{a c}^{a, b}$

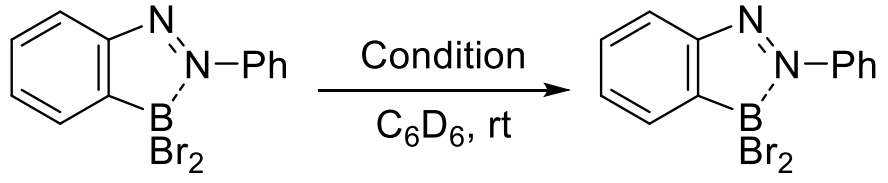

2a 2a
Condition:

$\mathrm{AcOH}$ (1 equiv.), $1 \mathrm{~h}$

$\mathrm{NEt}_{3}$ (1 equiv.), $1 \mathrm{~h}$

$\mathrm{H}_{2} \mathrm{O}(0.5 \mathrm{~mL}), 6 \mathrm{~h}$

Phosphate buffer solution ( $\mathrm{pH} 7.0,0.5 \mathrm{~mL}$ ), $6 \mathrm{~h}$

Room light, $6 \mathrm{~h}$<smiles>PC1=CC=CN(c2ccccc2)c2ccccc21</smiles><smiles>[H][R6](=O)c1ccc2c(c1)N(c1ccccc1)B(c1ccccc1)N2c1ccccc1</smiles>

4ac
Recovery of $\mathbf{2 a}$ :

quant.

$60 \%$

quant.

quant.

quant.

Condition:

$\mathrm{AcOH}$ (1 equiv.), $1 \mathrm{~h}$

Recovery of 4ac:

$\mathrm{NEt}_{3}$ (1 equiv.), $1 \mathrm{~h}$

quant.

quant.

quant.

$\mathrm{H}_{2} \mathrm{O}(0.5 \mathrm{~mL}), 6 \mathrm{~h}$

Phosphate buffer solution ( $\mathrm{pH} 7.0,0.5 \mathrm{~mL}$ ), $6 \mathrm{~h}$ quant.

quant.

${ }^{a} \mathbf{2 a}$ or $4 \mathbf{a c}(0.20 \mathrm{mmol}), \mathrm{C}_{6} \mathrm{D}_{6}(1.0 \mathrm{~mL}), \mathrm{rt}, 1-6 \mathrm{~h} .{ }^{b}$ Yields were determined by ${ }^{1} \mathrm{H}$ NMR spectroscopy using 1,1,2-trichloroethane as an internal standard.

Scheme S3. Scale up of borylation of $1 \mathbf{a}^{a}$<smiles>c1ccc(/N=N/c2ccccc2)cc1</smiles>

$1 \mathrm{a}$

$1.82 \mathrm{~g}, 10 \mathrm{mmol}$

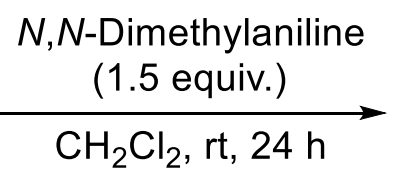

(3.0 equiv.)<smiles></smiles>

$2 \mathrm{a}$

$3.36 \mathrm{~g}, 95 \%$

${ }^{a}$ Isolated yield. 
Figure S1. Transformations of $\mathrm{BBr}_{2}$ moiety in $\mathbf{2 a}, \mathbf{2 g}$, and $\mathbf{2} \mathbf{h}^{a, b}$

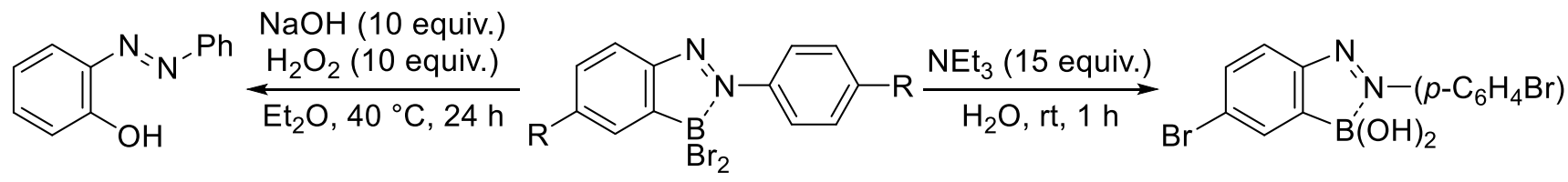

13a, $96 \%$

2a, 2g, $2 \mathbf{h}(\mathrm{R}=\mathrm{H}, \mathrm{Cl}, \mathrm{Br})$

$14 \mathrm{~h}, 95 \%$

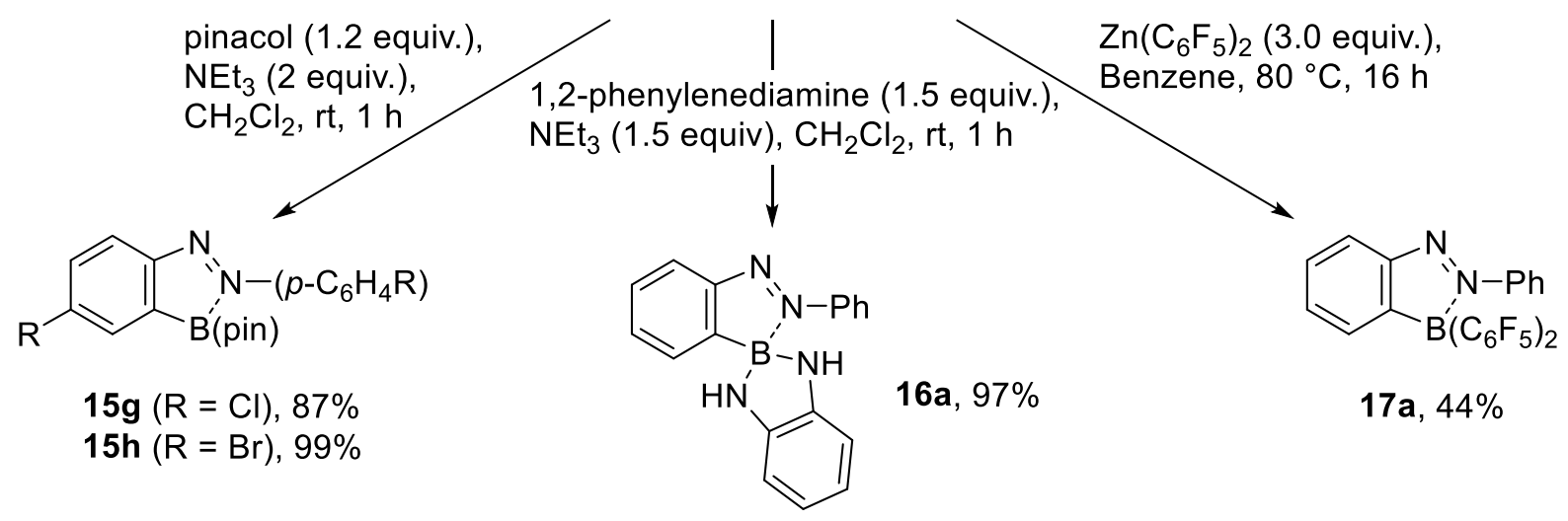

${ }^{a}$ Reactions were conducted on a $0.2 \mathrm{mmol}$ scale. ${ }^{b}$ Isolated yields.

Figure S2. UV-vis spectra of 1a, 2a, and 4ac in $\mathrm{CH}_{2} \mathrm{Cl}_{2}\left(0.5 \mathrm{mM}, 20{ }^{\circ} \mathrm{C}\right)$.

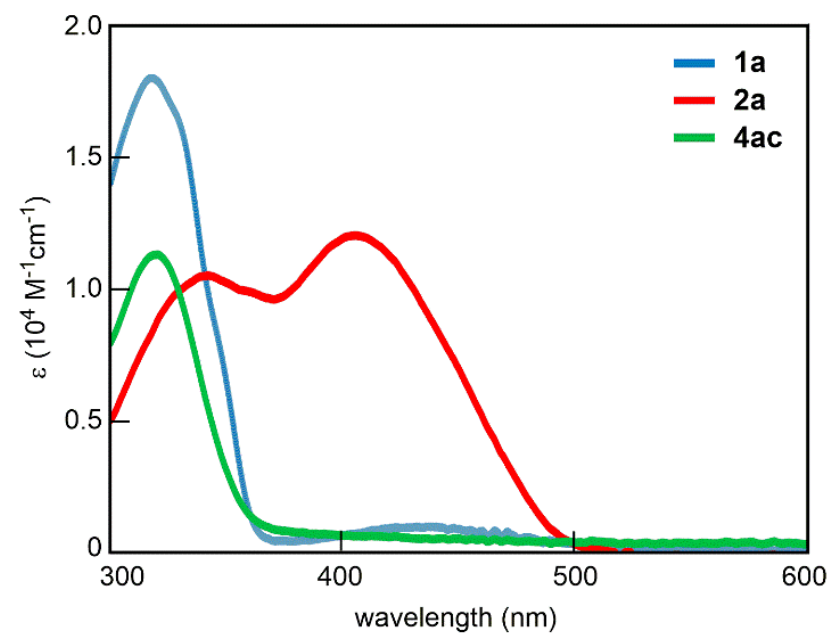


Scheme S4. Reaction of $1 \mathbf{a}$ with $\mathbf{3} \mathbf{a}^{a, b}$

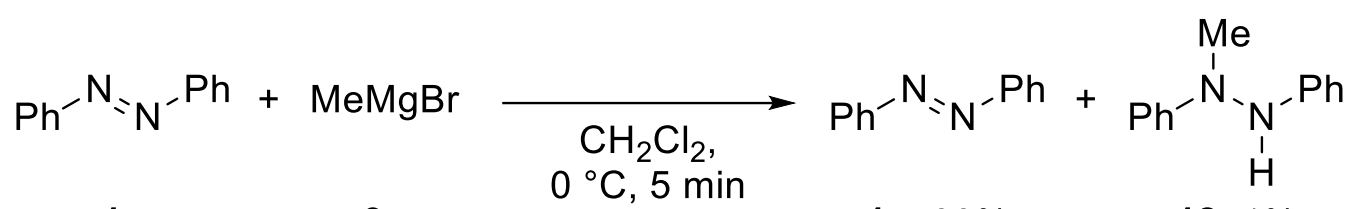

$1 \mathrm{a}$

$3 a$

$1 a, 99 \%$

$18,1 \%$

(2.5 equiv.)

${ }^{a}$ Reaction was conducted on a $0.2 \mathrm{mmol}$ scale. ${ }^{b}$ Yields were determined by ${ }^{1} \mathrm{H}$ NMR spectroscopy using 1,1,2-trichloroethane as an internal standard.

Table S3. Diethylative cyclization of $\mathbf{2 a}^{a}$

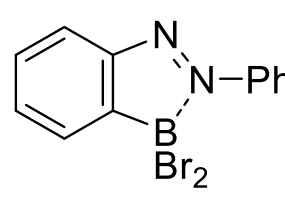

$2 a$

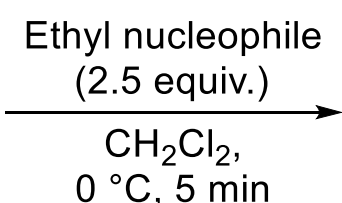

$0{ }^{\circ} \mathrm{C}, 5 \mathrm{~min}$<smiles>CCB1c2ccccc2N(CC)N1c1ccccc1</smiles>

$4 a b$

\begin{tabular}{|c|c|c|}
\hline Entry & Ethyl nucleophile & Yield of 4ab (\%) $^{b}$ \\
\hline 1 & EtMgBr & $(87)^{c}$ \\
\hline 2 & $\mathrm{AlEt}_{3}$ & 10 \\
\hline 3 & $\mathrm{ZnEt}_{2}$ & 7 \\
\hline
\end{tabular}

${ }^{a} \mathbf{a}(0.20 \mathrm{mmol})$, ethyl nucleophile $\left\{\mathrm{EtMgBr}(1.0 \mathrm{M}\right.$ in THF, $0.50 \mathrm{~mL}, 0.50 \mathrm{mmol}) ; \mathrm{AlEt}_{3}(1.0 \mathrm{M}$ in hexane, $0.50 \mathrm{~mL}, 0.50 \mathrm{mmol}) ; \mathrm{ZnEt}_{2}(1.12 \mathrm{M}$ in hexane, $\left.0.45 \mathrm{~mL}, 0.50 \mathrm{mmol})\right\}, \mathrm{CH}_{2} \mathrm{Cl}_{2}(0.50 \mathrm{~mL})$. ${ }^{b}$ Yields were determined by ${ }^{1} \mathrm{H}$ NMR spectroscopy using 1,1,2-trichloroethane as an internal standard. ${ }^{c}$ Yield in parentheses denotes the isolated yield. 
Figure S3. Comparison of ${ }^{1} \mathrm{H}$ NMR spectra ( $400 \mathrm{MHz}, \mathrm{C}_{6} \mathrm{D}_{6}$ ) of 5 and $\mathbf{6}$ obtained in the reactions of Schemes 1-(1) and 1-(2), respectively.

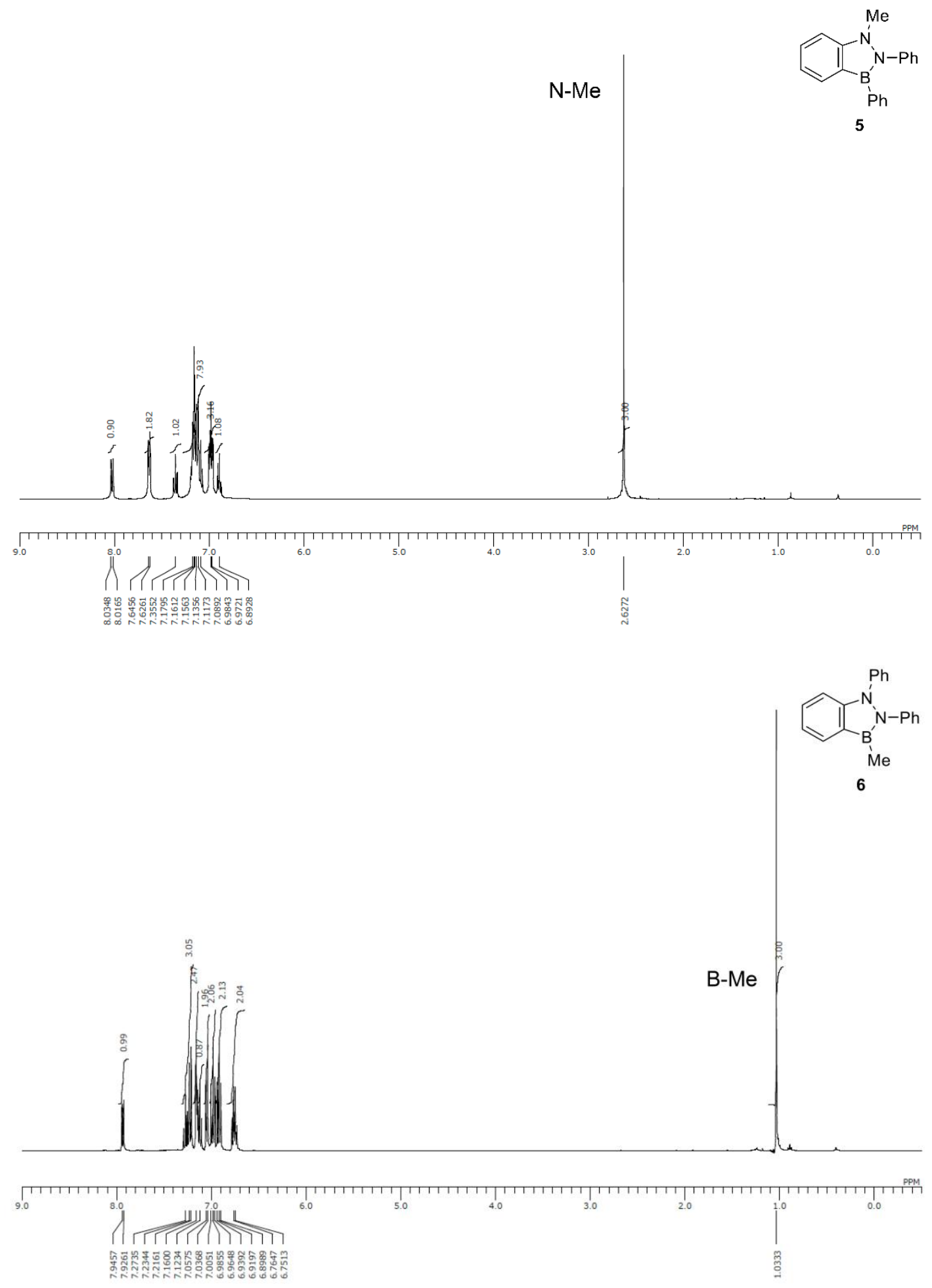


Materials. Unless otherwise noted, materials were purchased from Tokyo Kasei Co., Aldrich Inc. and other commercial suppliers and were used as received. $\mathbf{1} \mathbf{b}^{1}, \mathbf{1 c}^{2}, \mathbf{1 e}^{3}, \mathbf{1 f}^{2}, \mathbf{1 g}^{2}, \mathbf{1 h}^{2}, \mathbf{1 i}^{4}, \mathbf{1} \mathbf{j}^{1}$, and $\mathbf{1} \mathbf{k}^{5}$ were prepared according to the literature procedures. 1d and $\mathbf{1 l}$ were prepared according to the following Schemes S5 and S6, respectively. 3d, 3e, and $\mathbf{3 g}$ were prepared by slow addition of the corresponding aryl bromide in anhydrous THF to a mixture of $\mathrm{Mg}$ turnings and $\mathrm{I}_{2}$ (one crystal) in anhydrous THF, the concentrations of which were titrated by menthol and 1,10-phenanthroline. ${ }^{6}$ Flash column chromatography was performed with Kanto silica gel $60 \mathrm{~N}$ (spherical, neutral, 70-230 mesh).

Scheme S5. Preparation of 1d

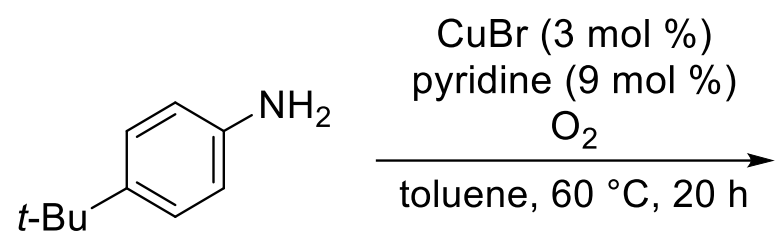

s1<smiles>CC(C)(C)c1ccc(/N=N/c2ccc(C(C)(C)C)cc2)cc1</smiles>

$1 d$

(E)-1,2-Bis(4-(tert-butyl)phenyl)diazene (1d). A mixture of $\mathrm{CuBr}(173.2 \mathrm{mg}, 1.21 \mathrm{mmol})$, pyridine (290 mL, $3.60 \mathrm{mmol}), \mathbf{S 1}(5.89 \mathrm{~g}, 39.5 \mathrm{mmol})$, and toluene $(160 \mathrm{~mL})$ was vigorously stirred under oxygen atmosphere $(1 \mathrm{~atm})$ at $60{ }^{\circ} \mathrm{C}$ in an oil bath for $20 \mathrm{~h}$. Then, the mixture was concentrated in vacuo. The residue was purified by silica gel chromatography (hexane:AcOEt $=10: 1$ ) to afford 1d (4.51 g, $15.3 \mathrm{mmol}, 78 \%$ ) as a red solid: $\mathrm{Mp} 188-192{ }^{\circ} \mathrm{C} .{ }^{1} \mathrm{H} \mathrm{NMR}\left(400 \mathrm{MHz}, \mathrm{CDCl}_{3}\right) \delta 7.84(\mathrm{~d}, J=$ $8.8 \mathrm{~Hz}, 4 \mathrm{H}), 7.52(\mathrm{~d}, J=8.8 \mathrm{~Hz}, 4 \mathrm{H}), 1.37$ (s, 18H). ${ }^{13} \mathrm{C} \mathrm{NMR}\left(100 \mathrm{MHz}, \mathrm{CDCl}_{3}\right) \delta 154.2,150.8$, 126.0, 122.4, 35.0, 31.3. LRMS (EI) $m / z: 294\left(\mathrm{M}^{+}\right)$. HRMS (EI-TOF) $m / z:\left(\mathrm{M}^{+}\right.$) Calcd. for $\mathrm{C}_{20} \mathrm{H}_{26} \mathrm{~N}_{2}$ : 294.2096, found: 294.2123. IR (neat): 845, 1010, 1107, 1161, 1268, 1362, 1458, 1602, 2867, $2959 \mathrm{~cm}^{-}$ 1.

Scheme S6. Preparation of 11<smiles>CC(C)(C)c1ccc(N)cc1</smiles>

S2
$\mathrm{AcOH}, 40^{\circ} \mathrm{C}, 12 \mathrm{~h}$<smiles>CC(C)(C)c1ccc(/N=N/c2ccccc2)cc1</smiles>

11

(E)-1-(4-(tert-Butyl)phenyl)-2-phenyldiazene (11). A mixture of S2 (1.60 g, $10.7 \mathrm{mmol})$ and nitrosobenzene $(1.29 \mathrm{~g}, 12.0 \mathrm{mmol})$ in acetic acid $(100 \mathrm{~mL})$ was stirred at $40{ }^{\circ} \mathrm{C}$ in an oil bath for 12 h. Then, $\mathrm{H}_{2} \mathrm{O}$ was added, and the organic materials were extracted with $\mathrm{CH}_{2} \mathrm{Cl}_{2}$. The combined organic layer was washed with $\mathrm{H}_{2} \mathrm{O}$, brine, dried over $\mathrm{Na}_{2} \mathrm{SO}_{4}$, and concentrated. The residue was purified by 
column chromatography on silica gel (hexane) to afford $\mathbf{1 l}(2.43 \mathrm{~g}, 10.2 \mathrm{mmol}, 95 \%)$ as an orange solid: $\mathrm{Mp} 47-49{ }^{\circ} \mathrm{C} .{ }^{1} \mathrm{H}$ NMR (400 MHz, $\left.\mathrm{CDCl}_{3}\right) \delta 7.92-7.83(\mathrm{~m}, 4 \mathrm{H}), 7.56-7.42(\mathrm{~m}, 5 \mathrm{H}), 1.37$ (s, 9H). ${ }^{13} \mathrm{C} \mathrm{NMR}\left(100 \mathrm{MHz}, \mathrm{CDCl}_{3}\right) \delta 154.6,152.9,150.7,130.7,129.0,126.0,122.7,122.6,35.0,31.3$. LRMS (EI) $m / z: 238\left(\mathrm{M}^{+}\right)$. HRMS (EI-EB) $m / z$ : $\left(\mathrm{M}^{+}\right.$) Calcd. for $\mathrm{C}_{16} \mathrm{H}_{18} \mathrm{~N}_{2}$ : 238.1470, found: 238.1467. IR (neat): 740, 768, 842, 849, 928, 1105, 1156, 1364, 1465, $2962 \mathrm{~cm}^{-1}$.

\section{General procedure of borylations of azobenzenes (Table 1 and Figure 2).}

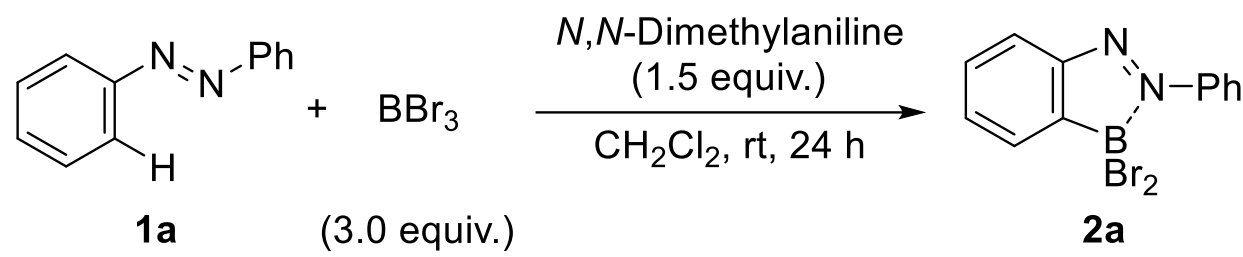

(E)-1-(2-(Dibromoboraneyl)phenyl)-2-phenyldiazene (2a). To a mixture of azobenzene 1a (36.4 mg, $0.200 \mathrm{mmol})$ and $N, N$-dimethylaniline $(40.8 \mathrm{mg}, 0.34 \mathrm{mmol}(38.0 \mu \mathrm{L}, 0.30 \mathrm{mmol}))$ was added $\mathrm{BBr}_{3}$ solution $\left(0.60 \mathrm{~mL}, 1.0 \mathrm{M}\right.$ in $\left.\mathrm{CH}_{2} \mathrm{Cl}_{2}, 0.60 \mathrm{mmol}\right)$ in an oven-dried vial equipped with a stirrer bar. After being stirred at room temperature for 24 hours, the solution was evaporated under reduced pressure. The residue was purified by passing through a short pad of silica gel (benzene) to give 2a (69.1 mg, $0.196 \mathrm{mmol}, 98 \%)$ as a red solid: $\mathrm{Mp} 182-187{ }^{\circ} \mathrm{C} .{ }^{1} \mathrm{H} \mathrm{NMR}\left(600 \mathrm{MHz}, \mathrm{CD}_{2} \mathrm{Cl}_{2}\right) \delta 8.63$ (dd, $J=7.3,1.9 \mathrm{~Hz}, 2 \mathrm{H}), 8.29(\mathrm{~d}, J=7.3 \mathrm{~Hz}, 1 \mathrm{H}), 7.83-7.75(\mathrm{~m}, 2 \mathrm{H}), 7.70-7.57(\mathrm{~m}, 4 \mathrm{H}) .{ }^{13} \mathrm{C}$ NMR $(150$ $\left.\mathrm{MHz}, \mathrm{CD}_{2} \mathrm{Cl}_{2}\right) \delta 153.8,142.1,137.3,134.2,130.2,129.9,129.7,128.8,124.9$ [carbon attached to boron was not observed due to quadrupole broadening caused by the boron nucleus]. ${ }^{11} \mathrm{~B}$ NMR (193 $\left.\mathrm{MHz}, \mathrm{CD}_{2} \mathrm{Cl}_{2} / \mathrm{BF}_{3} \cdot \mathrm{OEt}_{2}\right) \delta$-2.6. LRMS (EI) $m / z: 351\left(\mathrm{M}^{+}\right)$. HRMS (EI-TOF) $m / z:\left(\mathrm{M}^{+}\right)$Calcd. for $\mathrm{C}_{12} \mathrm{H}_{9} \mathrm{BBr}_{2} \mathrm{~N}_{2}: 349.9226$, found: 349.9240. IR (neat): 702, 720, 770, 826, 882, 922, 1145, 1163, 1229, $1507,1560 \mathrm{~cm}^{-1}$.<smiles></smiles>

(E)-1-(2-(Dibromoboraneyl)-6-methylphenyl)-2-(o-tolyl)diazene (2b). According to the general procedure analogous to that described for $\mathbf{2 a}, \mathbf{2 b}(73.6 \mathrm{mg}, 0.194 \mathrm{mmol}, 96 \%)$ was obtained from $\mathbf{1 b}$ (42.4 mg, $0.202 \mathrm{mmol})$ as a red solid: $\mathrm{Mp} 178-181{ }^{\circ} \mathrm{C} .{ }^{1} \mathrm{H} \mathrm{NMR}\left(600 \mathrm{MHz}, \mathrm{CD}_{2} \mathrm{Cl}_{2}\right) \delta 8.62(\mathrm{~d}, J=$ $7.3 \mathrm{~Hz}, 1 \mathrm{H}), 7.65(\mathrm{t}, J=7.3 \mathrm{~Hz}, 1 \mathrm{H}), 7.58(\mathrm{~d}, J=7.3 \mathrm{~Hz}, 1 \mathrm{H}), 7.53-7.43(\mathrm{~m}, 3 \mathrm{H}), 7.36(\mathrm{~d}, J=8.2 \mathrm{~Hz}$, $1 \mathrm{H}), 2.83(\mathrm{~s}, 3 \mathrm{H}), 2.44(\mathrm{~s}, 3 \mathrm{H}) .{ }^{13} \mathrm{C} \mathrm{NMR}\left(150 \mathrm{MHz}, \mathrm{CD}_{2} \mathrm{Cl}_{2}\right) \delta 153.2,142.8,141.2,138.0,134.6$, 133.1, 131.9, 131.4, 127.3, 127.0, 126.9, 20.4, 16.7 [carbon attached to boron was not observed due to quadrupole broadening caused by the boron nucleus]. ${ }^{11} \mathrm{~B} \mathrm{NMR}\left(193 \mathrm{MHz}, \mathrm{CD}_{2} \mathrm{Cl}_{2} / \mathrm{BF}_{3} \cdot \mathrm{OEt}_{2}\right) \delta-1.6$. 
LRMS (EI) $m / z: 379\left(\mathrm{M}^{+}\right)$. HRMS (EI-EB) $m / z:\left(\mathrm{M}^{+}\right)$Calcd. for $\mathrm{C}_{14} \mathrm{H}_{13} \mathrm{BBr}_{2} \mathrm{~N}_{2}$ : 377.9539, found: 377.9541. IR (neat): 702, 713, 746, 768, 1207, 1219, 1379, 1569, 2963, $2978 \mathrm{~cm}^{-1}$.<smiles>Cc1ccc(N2[B]c3cc(C)ccc3N2)cc1</smiles>

(E)-1-(2-(Dibromoboraneyl)-4-methylphenyl)-2-(p-tolyl)diazene (2c). According to the general procedure analogous to that described for $\mathbf{2 a}, \mathbf{2 c}(74.0 \mathrm{mg}, 0.195 \mathrm{mmol}, 98 \%)$ was obtained from 1c (42.0 mg, $0.200 \mathrm{mmol})$ as a red solid: $\mathrm{Mp} \mathrm{200-202}{ }^{\circ} \mathrm{C} .{ }^{1} \mathrm{H}$ NMR $\left(600 \mathrm{MHz}, \mathrm{CD}_{2} \mathrm{Cl}_{2}\right) \delta 8.47(\mathrm{~d}, J=$ $8.3 \mathrm{~Hz}, 2 \mathrm{H}), 8.12(\mathrm{~d}, J=8.3 \mathrm{~Hz}, 1 \mathrm{H}), 7.61(\mathrm{~s}, 1 \mathrm{H}), 7.42(\mathrm{~d}, J=8.2 \mathrm{~Hz}, 2 \mathrm{H}), 7.39(\mathrm{~d}, J=8.3 \mathrm{~Hz}, 1 \mathrm{H})$, $2.55(\mathrm{~s}, 3 \mathrm{H}), 2.51(\mathrm{~s}, 3 \mathrm{H}) .{ }^{13} \mathrm{C} \mathrm{NMR}\left(150 \mathrm{MHz}, \mathrm{CD}_{2} \mathrm{Cl}_{2}\right) \delta 152.3,149.6,145.3,140.0,131.1,130.50$, $130.47,128.3,124.4,22.8,21.8$ [carbon attached to boron was not observed due to quadrupole broadening caused by the boron nucleus]. ${ }^{11} \mathrm{~B} \mathrm{NMR}\left(193 \mathrm{MHz}, \mathrm{CD}_{2} \mathrm{Cl}_{2} / \mathrm{BF}_{3} \cdot \mathrm{OEt}_{2}\right) \delta-2.83$. LRMS (EI) $m / z: 380\left(\mathrm{M}^{+}\right)$. HRMS (EI-TOF) $m / z:\left(\mathrm{M}^{+}\right)$Calcd. for $\mathrm{C}_{14} \mathrm{H}_{13} \mathrm{BBr}_{2} \mathrm{~N}_{2}: 377.9539$, found: 377.9526 . IR (neat): 748, 818, 931, 1102, 1130, 1180, 1212, 1239, 1337, 1597, $2914 \mathrm{~cm}^{-1}$.<smiles></smiles>

(E)-1-(4-(tert-Butyl)-2-(dibromoboraneyl)phenyl)-2-(4-(tert-butyl)phenyl)diazene

(2d).

According to the general procedure analogous to that described for 2a, 2d (91.6 mg, $0.197 \mathrm{mmol}, 98 \%$ ) was obtained from 1d $(59.3 \mathrm{mg}, 0.201 \mathrm{mmol})$ as a red solid: Mp 194-199 ${ }^{\circ} \mathrm{C} .{ }^{1} \mathrm{H}$ NMR $(600 \mathrm{MHz}$, $\left.\mathrm{CD}_{2} \mathrm{Cl}_{2}\right) \delta 8.55-8.51(\mathrm{~m}, 2 \mathrm{H}), 8.16(\mathrm{~d}, J=8.2 \mathrm{~Hz}, 1 \mathrm{H}), 7.84(\mathrm{~d}, J=1.9 \mathrm{~Hz}, 1 \mathrm{H}), 7.66-7.61(\mathrm{~m}, 3 \mathrm{H})$, $1.42(\mathrm{~s}, 9 \mathrm{H}), 1.40(\mathrm{~s}, 9 \mathrm{H}) .{ }^{13} \mathrm{C}$ NMR $\left(150 \mathrm{MHz}, \mathrm{CD}_{2} \mathrm{Cl}_{2}\right) \delta 162.1,158.1,152.2,139.9,128.2,127.7$, $126.9,126.8,124.3,36.5,35.7,31.2,31.1$ [carbon attached to boron was not observed due to quadrupole broadening caused by the boron nucleus]. ${ }^{11} \mathrm{~B} \mathrm{NMR}\left(193 \mathrm{MHz}, \mathrm{CD}_{2} \mathrm{Cl}_{2} / \mathrm{BF}_{3} \cdot \mathrm{OEt}_{2}\right) \delta-2.7$. LRMS (EI) $m / z$ : $464\left(\mathrm{M}^{+}\right)$. HRMS (EI-TOF) $m / z$ : $\left(\mathrm{M}^{+}\right)$Calcd. for $\mathrm{C}_{20} \mathrm{H}_{25} \mathrm{BBr}_{2} \mathrm{~N}_{2}$ : 462.0478, found: 462.0458. IR (neat): 708, 763, 816, 827, 842, 1110, 1162, 1240, 1596, $2963 \mathrm{~cm}^{-1}$.<smiles></smiles> 
(E)-1-(2-(Dibromoboraneyl)-3,5-dimethylphenyl)-2-(3,5-dimethylphenyl)diazene (2e). According to the general procedure analogous to that described for $\mathbf{2 a}, \mathbf{2 e}(80.9 \mathrm{mg}, 0.198 \mathrm{mmol}, 96 \%)$ was obtained from 1e $(49.2 \mathrm{mg}, 0.206 \mathrm{mmol})$ as a red solid: $\mathrm{Mp} 245-246{ }^{\circ} \mathrm{C} .{ }^{1} \mathrm{H} \mathrm{NMR}\left(600 \mathrm{MHz}, \mathrm{CD}_{2} \mathrm{Cl}_{2}\right)$ $\delta 8.13(\mathrm{~s}, 2 \mathrm{H}), 7.83(\mathrm{~s}, 1 \mathrm{H}), 7.29(\mathrm{~s}, 1 \mathrm{H}), 7.20(\mathrm{~s}, 1 \mathrm{H}), 2.47(\mathrm{~s}, 3 \mathrm{H}), 2.40(\mathrm{~s}, 3 \mathrm{H}), 2.37(\mathrm{~s}, 6 \mathrm{H}) .{ }^{13} \mathrm{C}$ NMR (150 MHz, $\left.\mathrm{CD}_{2} \mathrm{Cl}_{2}\right) \delta 154.2,142.3,141.1,140.9,139.9,139.3,135.8,126.5,122.4,21.5,21.2$, 19.0 [carbon attached to boron was not observed due to quadrupole broadening caused by the boron nucleus]. ${ }^{11} \mathrm{~B}$ NMR (193 MHz, $\mathrm{CD}_{2} \mathrm{Cl}_{2} / \mathrm{BF}_{3} \cdot \mathrm{OEt}_{2}$ ) $\delta$-2.7. LRMS (EI) $m / z: 408\left(\mathrm{M}^{+}\right.$). HRMS (EI-TOF) $m / z$ : $\left(\mathrm{M}^{+}\right)$Calcd. for $\mathrm{C}_{16} \mathrm{H}_{17} \mathrm{BBr}_{2} \mathrm{~N}_{2}: 405.9852$, found: 405.9845. IR (neat): 751, 760, 838, 851, 909, $1165,1272,1289,1613,2919 \mathrm{~cm}^{-1}$.<smiles>Fc1ccc(N2[B]c3cc(F)ccc3N=N2)cc1</smiles>

(E)-1-(2-(Dibromoboraneyl)-4-fluorophenyl)-2-(4-fluorophenyl)diazene (2f). According to the general procedure analogous to that described for $\mathbf{2 a}, \mathbf{2 f}(77.8 \mathrm{mg}, 0.201 \mathrm{mmol}, 100 \%)$ was obtained from 1f (43.8 mg, $0.201 \mathrm{mmol})$ as a red solid: Mp 188-190 ${ }^{\circ} \mathrm{C} .{ }^{1} \mathrm{H}$ NMR $\left(600 \mathrm{MHz}, \mathrm{CD}_{2} \mathrm{Cl}_{2}\right) \delta$ 8.65-8.60 (m, 2H), $8.30(\mathrm{dd}, J=8.3,4.6 \mathrm{~Hz}, 1 \mathrm{H}), 7.47(\mathrm{dd}, J=7.4,2.7 \mathrm{~Hz}, 1 \mathrm{H}), 7.35-7.30(\mathrm{~m}, 2 \mathrm{H})$, $7.29-7.25(\mathrm{~m}, 1 \mathrm{H}) .{ }^{13} \mathrm{C} \mathrm{NMR}\left(150 \mathrm{MHz}, \mathrm{CD}_{2} \mathrm{Cl}_{2}\right) \delta 168.8\left(\mathrm{~d}, J_{\mathrm{CF}}=262.8 \mathrm{~Hz}\right), 166.0\left(\mathrm{~d}, J_{\mathrm{CF}}=255.6\right.$ $\mathrm{Hz}), 150.2,138.5,131.5\left(\mathrm{~d}, J_{\mathrm{CF}}=10.1 \mathrm{~Hz}\right), 127.2\left(\mathrm{~d}, J_{\mathrm{CF}}=8.6 \mathrm{~Hz}\right), 118.0\left(\mathrm{~d}, J_{\mathrm{CF}}=25.8 \mathrm{~Hz}\right), 117.21$ $\left(\mathrm{d}, J_{\mathrm{CF}}=23.0 \mathrm{~Hz}\right), 117.18\left(\mathrm{~d}, J_{\mathrm{CF}}=24.4 \mathrm{~Hz}\right)$ [carbon attached to boron was not observed due to quadrupole broadening caused by the boron nucleus]. ${ }^{11} \mathrm{~B} \mathrm{NMR}\left(193 \mathrm{MHz}, \mathrm{CD}_{2} \mathrm{Cl}_{2} / \mathrm{BF}_{3} \cdot \mathrm{OEt}_{2}\right) \delta-3.0$. LRMS (EI) $m / z: 387\left(\mathrm{M}^{+}\right)$. HRMS (EI-TOF) $m / z$ : $\left(\mathrm{M}^{+}\right)$Calcd. for $\mathrm{C}_{12} \mathrm{H}_{7} \mathrm{BBr}_{2} \mathrm{~F}_{2} \mathrm{~N}_{2}: 385.9037$, found: 385.9075. IR (neat): 749, 786, 843, 1086, 1155, 1240, 1273, 1500, 1576, $3113 \mathrm{~cm}^{-1}$.<smiles></smiles>

(E)-1-(4-Chloro-2-(dibromoboraneyl)phenyl)-2-(4-chlorophenyl)diazene (2g). According to the general procedure analogous to that described for $\mathbf{2 a}, \mathbf{2 g}(81.8 \mathrm{mg}, 0.194 \mathrm{mmol}, 97 \%)$ was obtained from $1 \mathrm{~g}$ (50.4 mg, $0.201 \mathrm{mmol})$ as a red solid: Mp 220-225 ${ }^{\circ} \mathrm{C} .{ }^{1} \mathrm{H}$ NMR $\left(600 \mathrm{MHz}, \mathrm{CD}_{2} \mathrm{Cl}_{2}\right) \delta$ 8.59-8.53 (m, 2H), $8.22(\mathrm{~d}, J=8.3 \mathrm{~Hz}, 1 \mathrm{H}), 7.77(\mathrm{~s}, 1 \mathrm{H}), 7.65-7.59(\mathrm{~m}, 2 \mathrm{H}), 7.58(\mathrm{~d}, J=8.3,1.9 \mathrm{~Hz}$, 1H). ${ }^{13} \mathrm{C}$ NMR $\left(150 \mathrm{MHz}, \mathrm{CD}_{2} \mathrm{Cl}_{2}\right) \delta 152.0,145.1,140.8,140.4,130.8,130.3,130.2,129.9,126.1$ [carbon attached to boron was not observed due to quadrupole broadening caused by the boron nucleus]. ${ }^{11} \mathrm{~B} \mathrm{NMR}\left(193 \mathrm{MHz}, \mathrm{CD}_{2} \mathrm{Cl}_{2} / \mathrm{BF}_{3} \cdot \mathrm{OEt}_{2}\right) \delta$-2.9. LRMS (EI) $m / z: 419\left(\mathrm{M}^{+}\right)$. HRMS (EI-TOF) 
$m / z:\left(\mathrm{M}^{+}\right)$Calcd. for $\mathrm{C}_{12} \mathrm{H}_{7} \mathrm{BBr}_{2} \mathrm{Cl}_{2} \mathrm{~N}_{2}$ :417.8446, found: 417.8476. IR (neat): 706, 750, 822, 830, 1060, $1150,1236,1419,1559,3093 \mathrm{~cm}^{-1}$.<smiles></smiles>

(E)-1-(4-Bromo-2-(dibromoboraneyl)phenyl)-2-(4-bromophenyl)diazene (2h). According to the general procedure analogous to that described for $\mathbf{2 a}$, except that $\mathrm{BBr}_{3}$ solution $(0.80 \mathrm{~mL}, 1.0 \mathrm{M}$ in $\left.\mathrm{CH}_{2} \mathrm{Cl}_{2}, 0.80 \mathrm{mmol}\right)$ was used, $2 \mathbf{h}(92.1 \mathrm{mg}, 0.181 \mathrm{mmol}, 92 \%)$ was obtained from $\mathbf{1 h}(67.1 \mathrm{mg}, 0.197$ mmol) as a red solid: $\mathrm{Mp} 253-255{ }^{\circ} \mathrm{C}\left(\mathrm{CH}_{2} \mathrm{Cl}_{2} /\right.$ hexane). ${ }^{1} \mathrm{H} \mathrm{NMR}\left(600 \mathrm{MHz}, \mathrm{CD}_{2} \mathrm{Cl}_{2}\right) \delta 8.50-8.45$ (m, $2 \mathrm{H}), 8.13(\mathrm{~d}, J=8.3 \mathrm{~Hz}, 1 \mathrm{H}), 7.95(\mathrm{~d}, J=1.9 \mathrm{~Hz}, 1 \mathrm{H}), 7.80-7.73(\mathrm{~m}, 3 \mathrm{H}) .{ }^{13} \mathrm{C} \mathrm{NMR}(150 \mathrm{MHz}$, $\left.\mathrm{CD}_{2} \mathrm{Cl}_{2}\right) \delta 152.3,140.8,134.7,133.8,133.4,133.3,129.9,129.6,126.2$ [carbon attached to boron was not observed due to quadrupole broadening caused by the boron nucleus]. ${ }^{11} \mathrm{~B}$ NMR (193 MHz, $\left.\mathrm{CD}_{2} \mathrm{Cl}_{2} / \mathrm{BF}_{3} \cdot \mathrm{OEt}_{2}\right) \delta$-3.0. LRMS (EI) $m / z: 509\left(\mathrm{M}^{+}\right)$. HRMS (EI-EB) $m / z$ : $\left(\mathrm{M}^{+}\right)$Calcd. for $\mathrm{C}_{12} \mathrm{H}_{7} \mathrm{BBr}_{4} \mathrm{~N}_{2}: 505.7436$, found: 505.7430. IR (neat): 737, 819, 827, 895, 1006, 1050, 1071, 1151, 1305, $1555,2360 \mathrm{~cm}^{-1}$.<smiles></smiles>

(E)-1-(2-(Dibromoboraneyl)-4-iodophenyl)-2-(4-iodophenyl)diazene (2i). According to the general procedure analogous to that described for $\mathbf{2 a}, \mathbf{2 i}(112.1 \mathrm{mg}, 0.186 \mathrm{mmol}, 92 \%)$ was obtained from $\mathbf{1 i}$ (88.0 mg, $0.203 \mathrm{mmol})$ as a red solid: $\mathrm{Mp} 274-278{ }^{\circ} \mathrm{C}\left(\mathrm{CH}_{2} \mathrm{Cl}_{2} /\right.$ hexane) ${ }^{1} \mathrm{H}$ NMR $\left(600 \mathrm{MHz}, \mathrm{CDCl}_{3}\right)$ $\delta 8.33-8.30(\mathrm{~m}, 2 \mathrm{H}), 8.20(\mathrm{~s}, 1 \mathrm{H}), 7.98-7.90(\mathrm{~m}, 4 \mathrm{H}) .{ }^{13} \mathrm{C} \mathrm{NMR}\left(150 \mathrm{MHz}, \mathrm{CDCl}_{3}\right) \delta 152.5,141.0$, $139.3,139.1,139.0,129.0,125.6,108.9,102.2$ [carbon attached to boron was not observed due to quadrupole broadening caused by the boron nucleus]. ${ }^{11} \mathrm{~B} \mathrm{NMR}\left(193 \mathrm{MHz}, \mathrm{CDCl}_{3} / \mathrm{BF}_{3} \cdot \mathrm{OEt}_{2}\right) \delta-3.1$. LRMS (EI) $m / z$ : $603\left(\mathrm{M}^{+}\right)$. HRMS (EI-TOF) $m / z$ : $\left(\mathrm{M}^{+}\right.$) Calcd. for $\mathrm{C}_{12} \mathrm{H}_{7} \mathrm{BBr}_{2} \mathrm{I}_{2} \mathrm{~N}_{2}$ : 601.7158 , found: 601.7160. IR (neat): 748, 812, 819, 889, 1002, 1055, 1147, 1232, 1303, $1551 \mathrm{~cm}^{-1}$.<smiles></smiles>

(E)-1-(2-(Dibromoboraneyl)-4-(trifluoromethoxy)phenyl)-2-(4-

(trifluoromethoxy)phenyl)diazene (2j). According to the general procedure analogous to that 
described for $\mathbf{2 a}, \mathbf{2 j}$ (97.5 mg, $0.188 \mathrm{mmol}, 92 \%)$ was obtained from $\mathbf{1 j}$ (71.3 $\mathrm{mg}, 0.204 \mathrm{mmol}$ ) as an orange solid: $\mathrm{Mp} 125-128{ }^{\circ} \mathrm{C} .{ }^{1} \mathrm{H} \mathrm{NMR}\left(600 \mathrm{MHz}, \mathrm{CD}_{2} \mathrm{Cl}_{2}\right) \delta 8.69-8.68(\mathrm{~m}, 2 \mathrm{H}), 8.36(\mathrm{~d}, J=8.2 \mathrm{~Hz}$, $1 \mathrm{H}), 7.61(\mathrm{~s}, 1 \mathrm{H}), 7.48(\mathrm{~d}, J=8.2 \mathrm{~Hz}, 2 \mathrm{H}), 7.42-7.40(\mathrm{~m}, 1 \mathrm{H}) .{ }^{13} \mathrm{C} \mathrm{NMR}\left(100 \mathrm{MHz}, \mathrm{C}_{6} \mathrm{D}_{6}\right) \delta 155.6$, $152.78,152.76,150.8,139.4,130.1,126.6,121.1,120.9,120.7\left(\mathrm{~d}, J_{\mathrm{CF}}=258.5 \mathrm{~Hz}\right), 120.6\left(\mathrm{q}, J_{\mathrm{CF}}=\right.$ $259.1 \mathrm{~Hz}$ ) [carbon attached to boron was not observed due to quadrupole broadening caused by the boron nucleus]. ${ }^{11} \mathrm{~B}$ NMR (193 MHz, $\left.\mathrm{CD}_{2} \mathrm{Cl}_{2} / \mathrm{BF}_{3} \cdot \mathrm{OEt}_{2}\right) \delta-2.8$. LRMS (EI) $m / z: 519\left(\mathrm{M}^{+}\right)$. HRMS (EI-EB) $m / z:\left(\mathrm{M}^{+}\right)$Calcd. for $\mathrm{C}_{14} \mathrm{H}_{7} \mathrm{BBr}_{2} \mathrm{~F}_{6} \mathrm{~N}_{2} \mathrm{O}_{2}: 517.8872$, found: 517.8897. IR (neat): 720, 758, 840, $1098,1164,1261,1316,1578,2360 \mathrm{~cm}^{-1}$.<smiles></smiles>

(E)-1-(2-(Dibromoboraneyl)-4-methylphenyl)-2-phenyldiazene (2k). According to the general procedure analogous to that described for $\mathbf{2 a}, \mathbf{2 k}(88.6 \mathrm{mg}, 0.242 \mathrm{mmol}, 99 \%)$ was obtained from $\mathbf{1 k}$ (48.0 mg, $0.245 \mathrm{mmol}$ ) as a red solid: $\mathrm{Mp} 172-175{ }^{\circ} \mathrm{C}\left(\mathrm{CH}_{2} \mathrm{Cl}_{2} /\right.$ hexane). ${ }^{1} \mathrm{H}$ NMR $\left(400 \mathrm{MHz}, \mathrm{CDCl}_{3}\right)$ $\delta 8.62-8.56(\mathrm{~m}, 2 \mathrm{H}), 8.11(\mathrm{~d}, J=7.8 \mathrm{~Hz}, 1 \mathrm{H}), 7.64(\mathrm{~s}, 1 \mathrm{H}), 7.62-7.57(\mathrm{~m}, 3 \mathrm{H}), 7.36(\mathrm{~d}, J=6.4 \mathrm{~Hz}$, $1 \mathrm{H}), 2.55(\mathrm{~s}, 3 \mathrm{H}) .{ }^{13} \mathrm{C} \mathrm{NMR}\left(150 \mathrm{MHz}, \mathrm{CDCl}_{3}\right) \delta 151.9,149.6,141.6,133.1,130.6,130.3,129.4$, 128.4, 124.2, 22.8 [carbon attached to boron was not observed due to quadrupole broadening caused by the boron nucleus]. ${ }^{11} \mathrm{~B}$ NMR (193 $\left.\mathrm{MHz}, \mathrm{CDCl}_{3} / \mathrm{BF}_{3} \cdot \mathrm{OEt}_{2}\right) \delta-2.9$. LRMS (EI) $m / z: 366\left(\mathrm{M}^{+}\right)$. HRMS (EI-TOF) $m / z:\left(\mathrm{M}^{+}\right)$Calcd. for $\mathrm{C}_{13} \mathrm{H}_{11} \mathrm{BBr}_{2} \mathrm{~N}_{2}: 363.9382$, found: 363.9407. IR (neat): 711, 764, $812,1215,1237,1356,1575,1599,3011,3068 \mathrm{~cm}^{-1}$.<smiles></smiles>

(E)-1-(4-(tert-Butyl)-2-(dibromoboraneyl)phenyl)-2-phenyldiazene (2l). According to the general procedure analogous to that described for $\mathbf{2 a}, \mathbf{2 l}(102.3 \mathrm{mg}, 0.251 \mathrm{mmol}, 98 \%)$ was obtained from $\mathbf{1 l}$ (60.8 mg, $0.255 \mathrm{mmol})$ as a red solid: $\mathrm{Mp} 188-191{ }^{\circ} \mathrm{C}\left(\mathrm{CH}_{2} \mathrm{Cl}_{2} /\right.$ hexane). ${ }^{1} \mathrm{H}$ NMR $\left(600 \mathrm{MHz}, \mathrm{CDCl}_{3}\right)$ $\delta 8.63-8.58(\mathrm{~m}, 2 \mathrm{H}), 8.15(\mathrm{~d}, J=8.3 \mathrm{~Hz}, 1 \mathrm{H}), 7.87(\mathrm{~d}, J=1.8 \mathrm{~Hz}, 1 \mathrm{H}), 7.63-7.57(\mathrm{~m}, 4 \mathrm{H}), 1.42(\mathrm{~s}$, 9H). ${ }^{13} \mathrm{C} \mathrm{NMR}\left(150 \mathrm{MHz}, \mathrm{CDCl}_{3}\right) \delta 162.1,151.8,141.6,133.1,129.3,128.1,127.2,126.5,124.2$, $36.3,31.1$ [carbon attached to boron was not observed due to quadrupole broadening caused by the boron nucleus]. ${ }^{11} \mathrm{~B}$ NMR (193 MHz, $\left.\mathrm{CDCl}_{3} / \mathrm{BF}_{3} \cdot \mathrm{OEt}_{2}\right) \delta$-2.8. LRMS (EI) $m / z: 408\left(\mathrm{M}^{+}\right)$. HRMS (EIEB) $m / z:\left(\mathrm{M}^{+}\right)$Calcd. for $\mathrm{C}_{16} \mathrm{H}_{17} \mathrm{BBr}_{2} \mathrm{~N}_{2}$ :405.9852, found: 405.9857. IR (neat): 702, 769, 825, 1162, 1240, 1360, 1472, 1597, 2864, 2967 $\mathrm{cm}^{-1}$. 


\section{Procedure of oxidation of 2a (Figure S1).}<smiles>BrC1=NB(c2ccccc2Br)c2ccccc21</smiles>

$2 a$

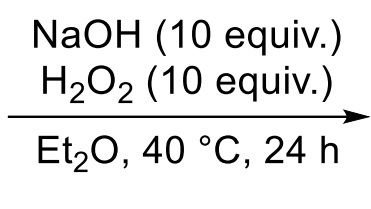

$\underset{\mathrm{Et}_{2} \mathrm{O}, 40^{\circ} \mathrm{C}, 24 \mathrm{~h}}{\mathrm{\textrm {H } _ { 2 }} \mathrm{O}_{2}(10 \text { equiv. })}$<smiles>Oc1ccccc1/N=N/c1ccccc1</smiles>

$13 a$

(E)-2-(Phenyldiazenyl)phenol (13a). A mixture of 2a (70.5 mg, $0.200 \mathrm{mmol}), \mathrm{NaOH}(80.2 \mathrm{mg}, 2.0$ $\mathrm{mmol}), \mathrm{H}_{2} \mathrm{O}_{2}(212.8 \mathrm{mg}, 2.2 \mathrm{mmol})$ in $\mathrm{Et}_{2} \mathrm{O}(1.0 \mathrm{~mL})$ was stirred in an oven-dried vial equipped with a stirrer bar. After being stirred at $40{ }^{\circ} \mathrm{C}$ in a heat block for 24 hours, the solution was neutralized with $1.0 \mathrm{M} \mathrm{HCl}$ and extracted with $\mathrm{CH}_{2} \mathrm{Cl}_{2}(10 \mathrm{~mL} \times 3)$. The combined organic layer was washed with $\mathrm{H}_{2} \mathrm{O}$ $(10 \mathrm{~mL})$ and brine $(10 \mathrm{~mL})$, dried over $\mathrm{Na}_{2} \mathrm{SO}_{4}$, and concentrated. The residue was purified by column chromatography on silica gel (hexane/AcOEt $=40: 1)$ to afford 13a $(38.0 \mathrm{mg}, 0.192 \mathrm{mmol}$, $96 \%$ yield) as an orange solid: $\mathrm{Mp} 77-79{ }^{\circ} \mathrm{C} .{ }^{1} \mathrm{H}$ NMR $\left(400 \mathrm{MHz}, \mathrm{CDCl}_{3}\right) \delta 12.9$ (s, 1H), 7.95 (dd, $J=8.3,1.9$ $\mathrm{Hz}, 1 \mathrm{H}), 7.88$ (dd, $J=8.3,1.5 \mathrm{~Hz}, 2 \mathrm{H}), 7.56-7.46(\mathrm{~m}, 3 \mathrm{H}), 7.38-7.33$ (m, 1H), 7.10-7.02 (m, 2H). ${ }^{13} \mathrm{C}$ NMR $\left(150 \mathrm{MHz}, \mathrm{CDCl}_{3}\right) \delta 152.8,150.5,137.4,133.3,133.2,131.1,129.3,122.2,119.9,118.2$. LRMS (EI) $\mathrm{m} / z$ : $198\left(\mathrm{M}^{+}\right)$. HRMS (EI-TOF) $\mathrm{m} / z$ : $\left(\mathrm{M}^{+}\right)$Calcd. for $\mathrm{C}_{12} \mathrm{H}_{10} \mathrm{~N}_{2} \mathrm{O}: 198.0793$, found: 198.0788. IR (neat): 736, 754, 768, 811, 1274, 1419, 1456, 1594, 2960, $3059 \mathrm{~cm}^{-1}$. The spectra data matched those reported in the literature. ${ }^{7}$

\section{Procedure of hydrolysis of $2 \mathrm{~h}$ (Figure S1).}

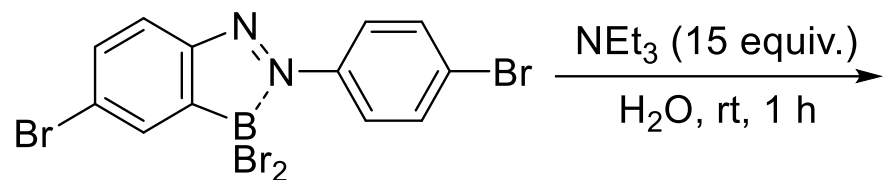

$2 \mathrm{~h}$<smiles>CO[Ga]c1cc(Br)ccc1-n1nnc2ccc(Br)cc21</smiles>

$14 h, 95 \%$

(E)-(5-Bromo-2-((4-bromophenyl)diazenyl)phenyl)boronic acid (14h). A mixture of $\mathbf{2 h}$ (101.4 mg, $0.199 \mathrm{mmol}), \mathrm{NEt}_{3}(303.6 \mathrm{mg})$, and $\mathrm{H}_{2} \mathrm{O}(0.4 \mathrm{~mL})$ was stirred at $0{ }^{\circ} \mathrm{C}$ in an oven-dried vial equipped with a stirrer bar. After being stirred at room temperature for $1 \mathrm{~h}$, the solution was neutralized with 1.0 $\mathrm{M} \mathrm{HCl}$. The resulting solid material was filtered and dried under vacuum to give $\mathbf{1 4 h}$ (72.4 $\mathrm{mg}, 0.189$ mmol, 95\%) as an orange solid: $\mathrm{Mp} 288-292^{\circ} \mathrm{C} .{ }^{1} \mathrm{H}$ NMR $\left(600 \mathrm{MHz}, \mathrm{DMSO}-d_{6}\right) \delta 7.90(\mathrm{~s}, 2 \mathrm{H})$, 7.85-7.77 (m, 5H), $7.73(\mathrm{~d}, J=7.3 \mathrm{~Hz}, 1 \mathrm{H}), 7.63(\mathrm{~d}, J=1.9 \mathrm{~Hz}, 1 \mathrm{H}) .{ }^{13} \mathrm{C}$ NMR (150 MHz, DMSOd6) $\delta 153.2,149.7,135.0,132.6,131.9,125.18,125.17,124.9,124.3$ [carbon attached to boron was not observed due to quadrupole broadening caused by the boron nucleus]. ${ }^{11} \mathrm{~B}$ NMR (DMSO$\left.d_{6} / \mathrm{BF}_{3} \cdot \mathrm{OEt}_{2}\right) \delta 31.1$. LRMS (EI) $m / z: 382\left(\mathrm{M}^{+}\right)$. HRMS (EI-EB) $m / z:\left(\mathrm{M}^{+}\right)$Calcd. for $\mathrm{C}_{12} \mathrm{H}_{9} \mathrm{BBr}_{2} \mathrm{~N}_{2} \mathrm{O}_{2}-$ :381.9124, found: 381.9137. IR (neat): 732, 756, 831, 1073, 1341, 1412, 1471, 1584, 3183, $3338 \mathrm{~cm}^{-}$ 1 . 
Procedure of protection of $2 \mathrm{~g}$ with pinacol (Figure S1).

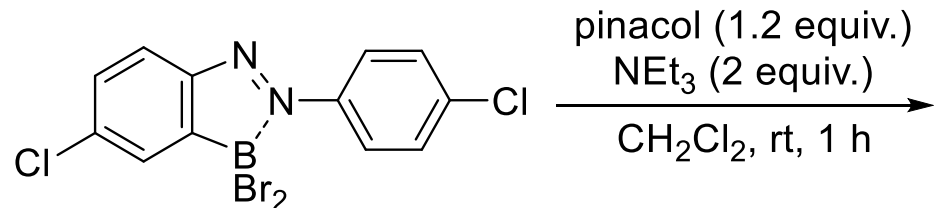

$2 \mathrm{~g}$<smiles></smiles>

$15 g, 87 \%$

(E)-1-(4-Chloro-2-(4,4,5,5-tetramethyl-1,3,2-dioxaborolan-2-yl)phenyl)-2-(4-

chlorophenyl)diazene (15g). To a mixture of $\mathbf{2 g}(83.5 \mathrm{mg}, 0.198 \mathrm{mmol})$ and $\mathrm{CH}_{2} \mathrm{Cl}_{2}(0.4 \mathrm{~mL})$ in an oven-dried vial equipped with a stirrer bar was added a solution of pinacol (28.4 $\mathrm{mg}, 0.24 \mathrm{mmol})$ and $\mathrm{NEt}_{3}(40.5 \mathrm{mg}, 0.4 \mathrm{mmol})$ in $\mathrm{CH}_{2} \mathrm{Cl}_{2}(1.5 \mathrm{~mL})$ at $0{ }^{\circ} \mathrm{C}$. After being stirred at room temperature for 1 $\mathrm{h}$, the solution was extracted with $\mathrm{CH}_{2} \mathrm{Cl}_{2}(10 \mathrm{~mL} \times 3)$. The combined organic layer was washed $\mathrm{H}_{2} \mathrm{O}$ and brine $(10 \mathrm{~mL})$, dried over $\mathrm{Na}_{2} \mathrm{SO}_{4}$, and concentrated in vacuo. The residue was purified by passing through a short pad of silica gel (AcOEt) to give $\mathbf{1 5 g}(64.8 \mathrm{mg}, 0.172 \mathrm{mmol}, 87 \%)$ as an orange solid: Mp 102-104 ${ }^{\circ} \mathrm{C}\left(\mathrm{CH}_{2} \mathrm{Cl}_{2} /\right.$ hexane). ${ }^{1} \mathrm{H}$ NMR (400 MHz, $\left.\mathrm{CDCl}_{3}\right) \delta 7.87-7.82(\mathrm{~m}, 2 \mathrm{H}), 7.74(\mathrm{~d}, J=8.8$ $\mathrm{Hz}), 7.70(\mathrm{~d}, J=2.4 \mathrm{~Hz}, 1 \mathrm{H}), 7.52-7.45(\mathrm{~m}, 3 \mathrm{H}), 1.36(\mathrm{~s}, 12 \mathrm{H}) .{ }^{13} \mathrm{C} \mathrm{NMR}\left(100 \mathrm{MHz}, \mathrm{CDCl}_{3}\right) \delta 155.0$, $151.1,136.9,136.5,134.4,130.9,129.3,124.3,120.7,84.3,25.0$ [carbon attached to boron was not observed due to quadrupole broadening caused by the boron nucleus]. ${ }^{11} \mathrm{~B} \mathrm{NMR}$ (193 $\mathrm{MHz}$, $\left.\mathrm{CDCl}_{3} / \mathrm{BF}_{3} \cdot \mathrm{OEt}_{2}\right) \quad \delta 30.8$. LRMS (EI) $m / z: 376\left(\mathrm{M}^{+}\right)$. HRMS (EI-TOF) $m / z$ : $\left(\mathrm{M}^{+}\right)$Calcd. for $\mathrm{C}_{18} \mathrm{H}_{19} \mathrm{BCl}_{2} \mathrm{~N}_{2} \mathrm{O}_{2}: 376.0917$, found: 376.0895. IR (neat): 715, 848, 1085, 1320, 1395, 1484, 2929, 2979 $\mathrm{cm}^{-1}$.<smiles></smiles>

(E)-1-(4-Bromo-2-(4,4,5,5-tetramethyl-1,3,2-dioxaborolan-2-yl)phenyl)-2-(4-

bromophenyl)diazene (15h). According to the procedure analogous to that described for $\mathbf{1 5 g}, \mathbf{1 5 h}$ (92.0 $\mathrm{mg}, 0.197 \mathrm{mmol}, 99 \%)$ was obtained from $\mathbf{2 h}(101.7 \mathrm{mg}, 0.200 \mathrm{mmol})$ as an orange solid: $\mathrm{Mp}$ $126-128{ }^{\circ} \mathrm{C}\left(\mathrm{CH}_{2} \mathrm{Cl}_{2} /\right.$ hexane $) .{ }^{1} \mathrm{H}$ NMR $\left(400 \mathrm{MHz}, \mathrm{CDCl}_{3}\right) \delta 7.86(\mathrm{~s}, 1 \mathrm{H}), 7.78(\mathrm{~d}, J=8.8 \mathrm{~Hz}, 2 \mathrm{H})$, 7.67-7.62 (m, 4H), $1.36(\mathrm{~s}, 12 \mathrm{H}) .{ }^{13} \mathrm{C} \mathrm{NMR}\left(100 \mathrm{MHz}, \mathrm{CDCl}_{3}\right) \delta 155.4,151.4,137.3,133.8,132.2$, $125.4,125.1,124.6,120.8,84.3,25.0$ [carbon attached to boron was not observed due to quadrupole broadening caused by the boron nucleus]. ${ }^{11} \mathrm{~B} \mathrm{NMR}\left(193 \mathrm{MHz}, \mathrm{CDCl}_{3} / \mathrm{BF}_{3} \cdot \mathrm{OEt}_{2}\right) \delta 30.8$. LRMS (EI) $m / z: 466\left(\mathrm{M}^{+}\right)$. HRMS (EI-TOF) $m / z:\left(\mathrm{M}^{+}\right)$Calcd. for $\mathrm{C}_{18} \mathrm{H}_{19} \mathrm{BBr}_{2} \mathrm{~N}_{2} \mathrm{O}_{2}: 463.9906$, found: 463.9909 . IR (neat): 702, 835, 1062, 1140, 1268, 1388, 1408, 1586, 2921, $2992 \mathrm{~cm}^{-1}$. 
Procedure of protection of 2a with 1,2-phenylenediamine (Figure S1).<smiles></smiles>

$2 \mathbf{a}$

$$
\begin{gathered}
\frac{\text { 1,2-phenylenediamine (1.5 equiv.) }}{\mathrm{NEt}_{3}(1.5 \text { equiv })} \\
\mathrm{CH}_{2} \mathrm{Cl}_{2}, \mathrm{rt}, 1 \mathrm{~h}
\end{gathered}
$$<smiles>c1ccc(-n2nnc3ccccc32)cc1</smiles>

$16 a$

(E)-2-(2-(Phenyldiazenyl)phenyl)-2,3-dihydro-1 $H$-benzo $[d][1,3,2]$ diazaborole (16a). To a mixture of $2 \mathbf{a}(70.6 \mathrm{mg}, 0.201 \mathrm{mmol})$ and $\mathrm{CH}_{2} \mathrm{Cl}_{2}(0.4 \mathrm{~mL})$ in an oven-dried vial equipped with a stirrer bar were added a solution of 1,2-phenylendiamine (34.3 $\mathrm{mg}, 0.32 \mathrm{mmol}$ ) and $\mathrm{NEt}_{3}(30.4 \mathrm{mg}, 0.30 \mathrm{mmol})$ in $\mathrm{CH}_{2} \mathrm{Cl}_{2}(1.5 \mathrm{~mL})$ at $0{ }^{\circ} \mathrm{C}$. After being stirred at room temperature for $1 \mathrm{~h}$, the solution was extracted with $\mathrm{CH}_{2} \mathrm{Cl}_{2}(10 \mathrm{~mL} \times 3)$. The combined organic layer was washed $\mathrm{H}_{2} \mathrm{O}$ and brine $(10 \mathrm{~mL})$, dried over $\mathrm{Na}_{2} \mathrm{SO}_{4}$, and concentrated in vacuo to give the crude product. After purification by flash chromatography on short silica gel, desired product $16 \mathbf{a}(57.8 \mathrm{mg}, 0.194 \mathrm{mmol}, 97 \%)$ was obtained as an orange solid. Mp: $138-140{ }^{\circ} \mathrm{C}\left(\mathrm{CH}_{2} \mathrm{Cl}_{2} /\right.$ hexane). ${ }^{1} \mathrm{H}$ NMR (400 MHz, $\left.\mathrm{CDCl}_{3}\right) \delta 7.93-7.88(\mathrm{~m}, 3 \mathrm{H})$, 7.83-7.77 (m, 1H), 7.61-7.48 (m, 3H), 7.16-7.06 (m, 4H), 7.00-6.94 (m, 2H). ${ }^{13} \mathrm{C}$ NMR (150 MHz, $\left.\mathrm{CDCl}_{3}\right) \delta 156.6,153.1,136.3,134.6,131.0,130.5,130.3,129.4,122.8,119.3,115.7,111.2$ [carbon attached to boron was not observed due to quadrupole broadening caused by the boron nucleus]. ${ }^{11} \mathrm{~B}$ NMR (193 MHz, $\left.\mathrm{CDCl}_{3} / \mathrm{BF}_{3} \cdot \mathrm{OEt}_{2}\right) \delta 28.4$. LRMS (EI) $m / z: 298\left(\mathrm{M}^{+}\right)$. HRMS (EI-TOF) $m / z:\left(\mathrm{M}^{+}\right)$ Calcd. for $\mathrm{C}_{18} \mathrm{H}_{15} \mathrm{BN}_{4}: 298.1390$, found: 298.1370. IR (neat): 733, 773, 1017, 1156, 1272, 1359, 1417 , $1599,2368,3055,3446,3464 \mathrm{~cm}^{-1}$. The spectra data matched those reported in the literature. ${ }^{8}$

\section{Procedure of diarylation of 2 a with $\mathrm{Zn}\left(\mathrm{C}_{6} \mathrm{~F}_{5}\right)_{2}$ (Figure S1).}<smiles></smiles>

$2 \mathbf{a}$

$$
\underset{\text { Benzene, } 80^{\circ} \mathrm{C}, 16 \mathrm{~h}}{\stackrel{\mathrm{Zn}\left(\mathrm{C}_{6} \mathrm{~F}_{5}\right)_{2}(3.0 \text { equiv. })}{\longrightarrow}}
$$<smiles></smiles>

$17 \mathbf{a}$

(E)-1-(2-(bis(perfluorophenyl)boraneyl)phenyl)-2-phenyldiazene (17a). A mixture of 2a (69.7 mg, $0.198 \mathrm{mmol}), \mathrm{Zn}\left(\mathrm{C}_{6} \mathrm{~F}_{5}\right)_{2}(240.9 \mathrm{mg}, 0.60 \mathrm{mmol})$, and benzene $(1.0 \mathrm{~mL})$ in an oven-dried vial equipped with a stirrer bar was stirred at $80{ }^{\circ} \mathrm{C}$ in a heat block for 16 hours. Then, $\mathrm{H}_{2} \mathrm{O}$ was added, and the organic materials were extracted with $\mathrm{CH}_{2} \mathrm{Cl}_{2}(10 \mathrm{~mL}$ x 3$)$. The combined organic layer was washed with $\mathrm{H}_{2} \mathrm{O}(10 \mathrm{~mL})$ and brine $(10 \mathrm{~mL})$, dried over $\mathrm{Na}_{2} \mathrm{SO}_{4}$, and concentrated. The combined organic layer was washed with brine, dried over $\mathrm{Na}_{2} \mathrm{SO}_{4}$, and concentrated. The residue was purified by column chromatography on silica gel (hexane/ $\left.\mathrm{CH}_{2} \mathrm{Cl}_{2}=4: 1\right)$ to give $17 \mathbf{a}(46.3 \mathrm{mg}, 0.088 \mathrm{mmol}, 44 \%)$ as a 
yellow solid: $\mathrm{Mp} 176-178{ }^{\circ} \mathrm{C} .{ }^{1} \mathrm{H}$ NMR $\left(600 \mathrm{MHz}, \mathrm{CD}_{2} \mathrm{Cl}_{2}\right) \delta 8.38(\mathrm{~d}, J=7.3 \mathrm{~Hz}, 1 \mathrm{H}), 8.06(\mathrm{~d}, J=$ $8.3 \mathrm{~Hz}, 2 \mathrm{H}), 7.68(\mathrm{~d}, J=7.3 \mathrm{~Hz}, 1 \mathrm{H}), 7.61(\mathrm{t}, J=7.3 \mathrm{~Hz}, 1 \mathrm{H}), 7.58-7.54(\mathrm{~m}, 2 \mathrm{H}), 7.53-7.48(\mathrm{~m}, 2 \mathrm{H})$. ${ }^{13} \mathrm{C}$ NMR (150 MHz, $\left.\mathrm{CD}_{2} \mathrm{Cl}_{2}\right) \delta 156.9,149.4-147.1(\mathrm{~m}), 144.3,139.9-138.5(\mathrm{~m}), 138.5-136.6(\mathrm{~m})$, $136.4,133.2,130.2,129.2,129.1,128.9,123.0$ [carbon attached to boron was not observed due to quadrupole broadening caused by the boron nucleus]. ${ }^{11} \mathrm{~B} \mathrm{NMR}\left(193 \mathrm{MHz}, \mathrm{CD}_{2} \mathrm{Cl}_{2} / \mathrm{BF}_{3} \cdot \mathrm{OEt}_{2}\right) \delta-0.6$. LRMS (EI) $m / z: 526\left(\mathrm{M}^{+}\right)$. HRMS (EI-TOF) $m / z:\left(\mathrm{M}^{+}\right)$Calcd. for $\mathrm{C}_{24} \mathrm{H}_{9} \mathrm{BF}_{10} \mathrm{~N}_{2}: 526.0699$, found: 526.0664. IR (neat): 745, 775, 978, 1107, 1146, 1375, 1456, 1517, 1645, $3068 \mathrm{~cm}^{-1}$. The spectra data matched those reported in the literature. ${ }^{9}$

\section{General procedure of dialkylative cyclizations of borylated azobenzenes to form BNN indoles} (Table 2 and Figures 2 and 3).<smiles></smiles>

$2 a$
$3 a$ (2.5 equiv.)

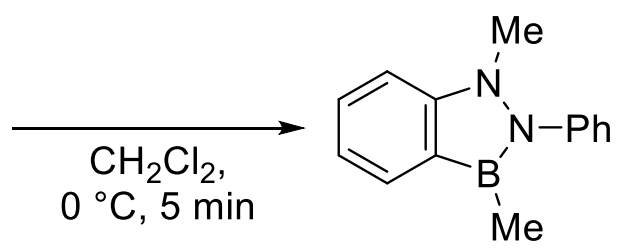

4aa

1,3-Dimethyl-2-phenyl-2,3-dihydro- $1 H$-benzo $[\boldsymbol{d}][\mathbf{1 , 2 , 3}]$ diazaborole (4aa). To a mixture of $2 \mathrm{a}$ (70.9 $\mathrm{mg}, 0.202 \mathrm{mmol})$ and $\mathrm{CH}_{2} \mathrm{Cl}_{2}(0.5 \mathrm{~mL})$ in an oven-dried vial equipped with a stirrer bar was added 3a solution $(0.167 \mathrm{~mL}, 3.0 \mathrm{M}$ in THF, $0.5 \mathrm{mmol})$ at $0{ }^{\circ} \mathrm{C}$. After being stirred at $0{ }^{\circ} \mathrm{C}$ for $5 \mathrm{~min}, \mathrm{H}_{2} \mathrm{O}(1$ $\mathrm{mL})$ was added, and the organic materials were extracted with $\mathrm{CH}_{2} \mathrm{Cl}_{2}(10 \mathrm{~mL} \times 3)$. The combined organic layer was washed with $\mathrm{H}_{2} \mathrm{O}(10 \mathrm{~mL})$ and brine $(10 \mathrm{~mL})$, dried over $\mathrm{Na}_{2} \mathrm{SO}_{4}$, and concentrated. The residue was purified by passing through a short pad of activated alumina (hexane: $\mathrm{CH}_{2} \mathrm{Cl}_{2}=5: 1$ ) to give $4 \mathbf{a a}(42.1 \mathrm{mg}, 0.190 \mathrm{mmol}, 94 \%)$ as a red oil. ${ }^{1} \mathrm{H} \mathrm{NMR}\left(600 \mathrm{MHz}, \mathrm{CD}_{2} \mathrm{Cl}_{2}\right) \delta 7.80(\mathrm{~d}, J=7.4$ $\mathrm{Hz}, 1 \mathrm{H}), 7.49-7.42(\mathrm{~m}, 3 \mathrm{H}), 7.33(\mathrm{~d}, J=7.3 \mathrm{~Hz}, 2 \mathrm{H}), 7.25-7.21(\mathrm{~m}, 2 \mathrm{H}), 7.11(\mathrm{t}, J=7.3 \mathrm{~Hz}, 1 \mathrm{H})$, 3.02 (s, 3H), 0.83 (s, 3H). ${ }^{13} \mathrm{C}$ NMR (150 MHz, $\left.\mathrm{CD}_{2} \mathrm{Cl}_{2}\right) \delta 157.1,141.6,131.3,130.1,129.2,125.2$, $125.0,121.1,112.4,39.5$ [carbon attached to boron was not observed due to quadrupole broadening caused by the boron nucleus]. ${ }^{11} \mathrm{~B} \mathrm{NMR}\left(193 \mathrm{MHz}, \mathrm{CD}_{2} \mathrm{Cl}_{2} / \mathrm{BF}_{3} \cdot \mathrm{OEt}_{2}\right) \delta 36.5$. LRMS (EI) $m / z: 222$ $\left(\mathrm{M}^{+}\right)$. HRMS (EI-TOF) $m / z:\left(\mathrm{M}^{+}\right)$Calcd. for $\mathrm{C}_{14} \mathrm{H}_{15} \mathrm{BN}_{2}: 222.1328$, found: 222.1327 . IR (neat): 735, $753,801,1124,1308,1451,1495,1598,2800,3016 \mathrm{~cm}^{-1}$.<smiles>CCN1c2ccccc2BBN1c1ccccc1</smiles>

Et 
1,3-Diethyl-2-phenyl-2,3-dihydro-1H-benzo $[d][1,2,3]$ diazaborole (4ab). According to the general procedure analogous to that described for $\mathbf{4 a a}$, except that the reaction was conducted with $\mathbf{3 b}$ solution $\left(0.167 \mathrm{~mL}, 3.0 \mathrm{M}\right.$ in $\left.\mathrm{Et}_{2} \mathrm{O}, 0.5 \mathrm{mmol}\right)$ and that the crude material was purified by passing through a short pad of activated alumina (hexane: $\left.\mathrm{CH}_{2} \mathrm{Cl}_{2}=1: 1\right)$, 4ab (43.4 mg, $0.173 \mathrm{mmol}, 87 \%$ ) was obtained from $2 \mathbf{a}(70.4 \mathrm{mg}, 0.200 \mathrm{mmol})$ as a red oil. ${ }^{1} \mathrm{H}$ NMR $\left(600 \mathrm{MHz}, \mathrm{C}_{6} \mathrm{D}_{6}\right) \delta 8.05(\mathrm{~d}, J=8.2 \mathrm{~Hz}, 1 \mathrm{H})$, $7.40(\mathrm{t}, J=6.9 \mathrm{~Hz}, 1 \mathrm{H}), 7.27(\mathrm{~d}, J=8.3 \mathrm{~Hz}, 2 \mathrm{H}), 7.19-7.15(\mathrm{~m}, 2 \mathrm{H}), 7.13(\mathrm{t}, J=7.4 \mathrm{~Hz}, 1 \mathrm{H}), 7.05(\mathrm{~d}$, $J=8.2 \mathrm{~Hz}, 1 \mathrm{H}), 7.00(\mathrm{t}, J=7.4 \mathrm{~Hz}, 1 \mathrm{H}), 3.46(\mathrm{q}, J=7.0 \mathrm{~Hz}, 2 \mathrm{H}), 1.51$ (q, $J=7.9 \mathrm{~Hz}, 2 \mathrm{H}), 1.31$ (t, $J$ $=7.8 \mathrm{~Hz}, 3 \mathrm{H}), 0.42(\mathrm{t}, J=6.9 \mathrm{~Hz}, 3 \mathrm{H}) .{ }^{13} \mathrm{C} \mathrm{NMR}\left(150 \mathrm{MHz}, \mathrm{C}_{6} \mathrm{D}_{6}\right) \delta 154.9,141.5,132.1,129.8,129.1$, $125.4,125.3,120.8,112.4,43.7,10.7,7.9$ [carbon attached to boron was not observed due to quadrupole broadening caused by the boron nucleus]. ${ }^{11} \mathrm{~B} \mathrm{NMR}\left(193 \mathrm{MHz}, \mathrm{C}_{6} \mathrm{D}_{6} / \mathrm{BF}_{3} \cdot \mathrm{OEt}_{2}\right) \delta 36.5$. LRMS (EI) $m / z$ : $250\left(\mathrm{M}^{+}\right)$. HRMS (EI-TOF) $m / z$ : $\left(\mathrm{M}^{+}\right)$Calcd. for $\mathrm{C}_{16} \mathrm{H}_{19} \mathrm{BN}_{2}: 250.1641$, found: 250.1622. IR (neat): 720, 746, 1123, 1307, 1453, 1460, 1495, 1598, 2955, $3069 \mathrm{~cm}^{-1}$.<smiles></smiles>

1,2,3-Triphenyl-2,3-dihydro-1H-benzo[d][1,2,3]diazaborole (4ac). According to the general procedure analogous to that described for $4 \mathbf{a a}$, except that the reaction was conducted with $\mathbf{3 c}$ solution $(0.250 \mathrm{~mL}, 2.0 \mathrm{M}$ in THF, $0.5 \mathrm{mmol})$ and that the crude material was purified by passing through a short pad of activated alumina (hexane: $\left.\mathrm{CH}_{2} \mathrm{Cl}_{2}=1: 1,0: 1\right)$, 4ac (55.9 $\left.\mathrm{mg}, 0.161 \mathrm{mmol}, 80 \%\right)$ was obtained from $2 \mathbf{a}(71.3 \mathrm{mg}, 0.202 \mathrm{mmol})$ as a colorless solid: Mp $159-160{ }^{\circ} \mathrm{C}\left(\mathrm{Et}_{2} \mathrm{O} /\right.$ hexane $) .{ }^{1} \mathrm{H} \mathrm{NMR}$ $\left(400 \mathrm{MHz}, \mathrm{CDCl}_{3}\right) \delta 7.98(\mathrm{~d}, J=7.8 \mathrm{~Hz}, 1 \mathrm{H}), 7.58-7.55(\mathrm{~m}, 2 \mathrm{H}), 7.43-7.38(\mathrm{~m}, 1 \mathrm{H}), 7.37-7.31(\mathrm{~m}$, 3H), 7.31-7.26 (m, 2H), 7.25-7.20 (m, 4H), 7.19-7.12 (m, 5H), $7.05(\mathrm{t}, J=7.3 \mathrm{~Hz}, 1 \mathrm{H}) .{ }^{13} \mathrm{C}$ NMR $\left(100 \mathrm{MHz}, \mathrm{CDCl}_{3}\right) \delta 154.9,142.9,140.4,134.1,131.5,130.0,128.9,128.45,128.42,127.7,127.0$, $126.5,126.3,125.5,121.4,112.0$ [carbon attached to boron was not observed due to quadrupole broadening caused by the boron nucleus]. ${ }^{11} \mathrm{~B} \mathrm{NMR}\left(193 \mathrm{MHz}, \mathrm{CDCl}_{3} / \mathrm{BF}_{3} \cdot \mathrm{OEt}_{2}\right) \delta 34.0$. LRMS (EI) $m / z: 346\left(\mathrm{M}^{+}\right)$. HRMS (EI-TOF) $m / z$ : $\left(\mathrm{M}^{+}\right)$Calcd. for $\mathrm{C}_{24} \mathrm{H}_{19} \mathrm{BN}_{2}$ : 346.1641, found: 346.1653. IR (neat): $753,764,788,1024,1274,1448,1489,1591,3033,3068 \mathrm{~cm}^{-1}$. 
<smiles>Cc1ccc(B2c3ccccc3N(c3ccc(C)cc3)N2c2ccccc2)cc1</smiles>

2-Phenyl-1,3-di-p-tolyl-2,3-dihydro- $1 H$-benzo $[d][1,2,3]$ diazaborole (4ad). According to the general procedure analogous to that described for 4aa, except that the reaction was conducted with $\mathbf{3 d}$ solution $(0.75 \mathrm{~mL}, 0.67 \mathrm{M}$ in THF, $0.50 \mathrm{mmol})$ and $\mathrm{CH}_{2} \mathrm{Cl}_{2}(1.0 \mathrm{~mL})$ at $-20{ }^{\circ} \mathrm{C}$ for $3 \mathrm{~min}$ and that the crude material was purified by passing through a short pad of activated alumina (hexane: $\mathrm{CH}_{2} \mathrm{Cl}_{2}=$ 4:1), 4ad (60.4 mg, $0.161 \mathrm{mmol}, 79 \%)$ was obtained from $\mathbf{2 a}(71.5 \mathrm{mg}, 0.203 \mathrm{mmol})$ as a colorless solid: $\mathrm{Mp} 132-135{ }^{\circ} \mathrm{C}\left(\mathrm{CH}_{2} \mathrm{Cl}_{2} /\right.$ hexane). ${ }^{1} \mathrm{H}$ NMR (400 MHz, $\left.\mathrm{C}_{6} \mathrm{D}_{6}\right) \delta 8.21(\mathrm{~d}, J=7.3 \mathrm{~Hz}, 1 \mathrm{H}), 7.76$ $(\mathrm{d}, J=7.8 \mathrm{~Hz}, 2 \mathrm{H}), 7.37-7.30(\mathrm{~m}, 1 \mathrm{H}), 7.26(\mathrm{~d}, J=8.3 \mathrm{~Hz}, 2 \mathrm{H}), 7.22-7.11(\mathrm{~m}, 4 \mathrm{H}), 6.99(\mathrm{~d}, J=7.8$ $\mathrm{Hz}, 2 \mathrm{H}), 6.91$ (t, $J=7.8 \mathrm{~Hz}, 2 \mathrm{H}), 6.76-6.70(\mathrm{~m}, 3 \mathrm{H}), 2.17(\mathrm{~s}, 3 \mathrm{H}), 1.86(\mathrm{~s}, 3 \mathrm{H}) .{ }^{13} \mathrm{C}$ NMR $(150 \mathrm{MHz}$, $\left.\mathrm{C}_{6} \mathrm{D}_{6}\right) \delta 156.0,141.2,141.0,138.4,136.8,134.7,132.0,130.2,129.8,129.1,128.6,127.0,126.7,125.7$, $121.8,112.6,21.5,20.8$ [carbon attached to boron was not observed due to quadrupole broadening caused by the boron nucleus]. ${ }^{11} \mathrm{~B}$ NMR $\left(193 \mathrm{MHz}, \mathrm{C}_{6} \mathrm{D}_{6} / \mathrm{BF}_{3} \cdot \mathrm{OEt}_{2}\right) \delta 33.5$. LRMS (EI) m/z: $374\left(\mathrm{M}^{+}\right)$. HRMS (EI-EB) $m / z$ : $\left(\mathrm{M}^{+}\right.$) Calcd. for $\mathrm{C}_{26} \mathrm{H}_{23} \mathrm{BN}_{2}: 374.1954$, found: 374.1950 . IR (neat): 714, 757, 1150, $1318,1452,1602,2366,2931,2979,3026 \mathrm{~cm}^{-1}$.<smiles>COc1ccc(B2c3ccccc3N(c3ccc(OC)cc3)N2c2ccccc2)cc1</smiles>

1,3-Bis(4-methoxyphenyl)-2-phenyl-2,3-dihydro-1H-benzo[d][1,2,3]diazaborole (4ae). According to the general procedure analogous to that described for 4aa, except that the reaction was conducted with $3 e$ solution $(0.64 \mathrm{~mL}, 0.79 \mathrm{M}$ in THF, $0.51 \mathrm{mmol})$ and $\mathrm{CH}_{2} \mathrm{Cl}_{2}(1.0 \mathrm{~mL})$ at $-20{ }^{\circ} \mathrm{C}$ for $3 \mathrm{~min}$ and 
that the crude material was purified by passing through a short pad of activated alumina $\left(\mathrm{CH}_{2} \mathrm{Cl}_{2}\right), 4 \mathbf{a e}$ (68.1 mg, $0.168 \mathrm{mmol}, 82 \%)$ was obtained from $\mathbf{2 a}(71.9 \mathrm{mg}, 0.204 \mathrm{mmol})$ as a colorless solid: $\mathrm{Mp}$ $148-150{ }^{\circ} \mathrm{C}\left(\mathrm{CH}_{2} \mathrm{Cl}_{2} /\right.$ hexane $) .{ }^{1} \mathrm{H} \mathrm{NMR}\left(400 \mathrm{MHz}, \mathrm{C}_{6} \mathrm{D}_{6}\right) \delta 8.24(\mathrm{~d}, J=7.3 \mathrm{~Hz}, 1 \mathrm{H}), 7.78(\mathrm{~d}, J=8.8$ $\mathrm{Hz}, 2 \mathrm{H}), 7.35$ (t, $J=7.8 \mathrm{~Hz}, 1 \mathrm{H}), 7.26$ (d, $J=8.3 \mathrm{~Hz}, 2 \mathrm{H}), 7.22-7.13(\mathrm{~m} 2 \mathrm{H}), 7.00-6.92(\mathrm{~m} 4 \mathrm{H}), 6.89$ $(\mathrm{d}, J=8.8 \mathrm{~Hz}, 2 \mathrm{H}), 6.77(\mathrm{t}, J=7.6 \mathrm{~Hz}, 1 \mathrm{H}), 6.50(\mathrm{~d}, J=8.8 \mathrm{~Hz}, 2 \mathrm{H}), 3.33(\mathrm{~s}, 3 \mathrm{H}), 3.06(\mathrm{~s}, 3 \mathrm{H}) .{ }^{13} \mathrm{C}$ $\operatorname{NMR}\left(150 \mathrm{MHz}, \mathrm{C}_{6} \mathrm{D}_{6}\right) \delta 160.9,158.9,156.4,141.3,136.3,136.2,132.0,130.1,128.7,128.4,127.5$, $125.8,121.7,114.4,114.1,112.8,54.6,54.5$ [carbon attached to boron was not observed due to quadrupole broadening caused by the boron nucleus]. ${ }^{11} \mathrm{~B} \mathrm{NMR}\left(193 \mathrm{MHz}, \mathrm{C}_{6} \mathrm{D}_{6} / \mathrm{BF}_{3} \cdot \mathrm{OEt}_{2}\right) \delta 33.1$. LRMS (EI) $m / z$ : $406\left(\mathrm{M}^{+}\right)$. HRMS (EI-EB) $m / z:\left(\mathrm{M}^{+}\right)$Calcd. for $\mathrm{C}_{26} \mathrm{H}_{23} \mathrm{BN}_{2} \mathrm{O}_{2}$ : 406.1853, found: 406.1836. IR (neat): 834, 1024, 1031, 1181, 1452, 1506, 1603, 2838, $3036 \mathrm{~cm}^{-1}$.<smiles>Clc1ccc(B2c3ccccc3N(c3ccccc3)N2c2ccc(Cl)cc2)cc1</smiles>

1,3-Bis(4-chlorophenyl)-2-phenyl-2,3-dihydro-1H-benzo[d][1,2,3]diazaborole (4af). According to the general procedure analogous to that described for 4aa, except that the reaction was conducted with 3f solution $\left(0.50 \mathrm{~mL}, 1.0 \mathrm{M}\right.$ in $\left.\mathrm{Et}_{2} \mathrm{O}, 0.5 \mathrm{mmol}\right)$ and $\mathrm{CH}_{2} \mathrm{Cl}_{2}-\mathrm{THF}(1: 1$, total $1.0 \mathrm{~mL})$ at $-20{ }^{\circ} \mathrm{C}$ and that the crude material was purified by passing through a short pad of activated alumina (hexane: $\left.\mathrm{CH}_{2} \mathrm{Cl}_{2}=10: 1\right)$ and by recrystallization $\left(\mathrm{CH}_{2} \mathrm{Cl}_{2} /\right.$ hexane), 4 af (39.4 $\mathrm{mg}, 0.0949 \mathrm{mmol}, 47 \%$ ) was obtained from $\mathbf{2 a}(70.5 \mathrm{mg}, 0.200 \mathrm{mmol})$ as a colorless solid: $\mathrm{Mp} 94-96{ }^{\circ} \mathrm{C}\left(\mathrm{CH}_{2} \mathrm{Cl}_{2} /\right.$ hexane $) .{ }^{1} \mathrm{H}$ NMR (400 MHz, $\left.\mathrm{C}_{6} \mathrm{D}_{6}\right) \delta 8.02(\mathrm{~d}, J=7.8 \mathrm{~Hz}, 1 \mathrm{H}), 7.50(\mathrm{~d}, J=8.3 \mathrm{~Hz}, 2 \mathrm{H}), 7.37-7.32(\mathrm{~m}, 1 \mathrm{H}), 7.24$ $(\mathrm{d}, J=8.8 \mathrm{~Hz}, 2 \mathrm{H}), 7.18-7.15(\mathrm{~m}, 1 \mathrm{H}), 7.06-7.00(\mathrm{~m}, 3 \mathrm{H}), 6.92-6.85(\mathrm{~m}, 4 \mathrm{H}), 6.76(\mathrm{~d}, J=7.6 \mathrm{~Hz}$, $1 \mathrm{H}), 6.72(\mathrm{~d}, J=8.8 \mathrm{~Hz}, 2 \mathrm{H}) .{ }^{13} \mathrm{C}$ NMR $\left(150 \mathrm{MHz}, \mathrm{C}_{6} \mathrm{D}_{6}\right) \delta 155.3,141.8,140.3,135.8,135.4,132.9$, $131.7,130.6,129.4,128.9,128.5,127.9,126.7,126.2,122.2,112.4$ [carbon attached to boron was not observed due to quadrupole broadening caused by the boron nucleus]. ${ }^{11} \mathrm{~B} \mathrm{NMR}$ (193 $\mathrm{MHz}$, $\left.\mathrm{C}_{6} \mathrm{D}_{6} / \mathrm{BF}_{3} \cdot \mathrm{OEt}_{2}\right) \delta$ 33.1. LRMS (EI) $m / z: 414\left(\mathrm{M}^{+}\right)$. HRMS (EI-TOF) $m / z:\left(\mathrm{M}^{+}\right)$Calcd. for $\mathrm{C}_{24} \mathrm{H}_{17} \mathrm{BCl}_{2} \mathrm{~N}_{2}$ : 414.0862, found: 414.0848. IR (neat): 753, 821, 1015, 1310, 1452, 1487, 1587, 2362, 2866, 2957, $3045 \mathrm{~cm}^{-1}$. 
<smiles>FC(F)(F)c1ccc(B2c3ccccc3N(c3ccccc3)N2c2ccc(C(F)(F)F)cc2)cc1</smiles>

2-Phenyl-1,3-bis(4-(trifluoromethyl)phenyl)-2,3-dihydro-1H-benzo[ $d][1,2,3]$ diazaborole (4ag). According to the general procedure analogous to that described for 4aa, except that the reaction was conducted with $3 \mathrm{~g}$ solution $(0.55 \mathrm{~mL}, 0.80 \mathrm{M}$ in THF, $0.44 \mathrm{mmol})$ and $\mathrm{CH}_{2} \mathrm{Cl}_{2}(1.0 \mathrm{~mL})$ at $-20{ }^{\circ} \mathrm{C}$ and that the crude material was purified by passing through a short pad of activated alumina (hexane: $\left.\mathrm{CH}_{2} \mathrm{Cl}_{2}=1: 1\right), 4 \mathbf{a g}(59.0 \mathrm{mg}, 0.122 \mathrm{mmol}, 61 \%)$ was obtained from 2a (70.7 mg, $\left.0.201 \mathrm{mmol}\right)$ as a colorless solid: $\mathrm{Mp} 147-150{ }^{\circ} \mathrm{C}\left(\mathrm{CH}_{2} \mathrm{Cl}_{2} /\right.$ hexane). ${ }^{1} \mathrm{H}$ NMR (400 MHz, $\left.\mathrm{C}_{6} \mathrm{D}_{6}\right) \delta 7.95(\mathrm{~d}, J=7.8$ $\mathrm{Hz}, 1 \mathrm{H}), 7.55$ (d, $J=8.3 \mathrm{~Hz}, 2 \mathrm{H}), 7.43$ (d, $J=7.8 \mathrm{~Hz}, 2 \mathrm{H}), 7.31$ (t, $J=7.8 \mathrm{~Hz}, 1 \mathrm{H}), 7.15-7.10$ (m, $3 \mathrm{H}), 7.02(\mathrm{~d}, J=9.3 \mathrm{~Hz}, 1 \mathrm{H}), 6.96(\mathrm{~d}, J=8.3 \mathrm{~Hz}, 2 \mathrm{H}), 6.86-6.78(\mathrm{~m}, 4 \mathrm{H}), 6.70(\mathrm{t}, J=7.3 \mathrm{~Hz}, 1 \mathrm{H})$. ${ }^{13} \mathrm{C} \mathrm{NMR}\left(100 \mathrm{MHz}, \mathrm{CDCl}_{3}\right) \delta 154.2,145.9,139.7,134.2,131.4,130.7,130.5\left(\mathrm{q}, J_{\mathrm{CF}}=32.1 \mathrm{~Hz}\right)$, $128.96\left(\mathrm{q}, J_{\mathrm{CF}}=32.9 \mathrm{~Hz}\right), 128.94,126.3\left(\mathrm{q}, J_{\mathrm{CF}}=3.8 \mathrm{~Hz}\right), 126.2,126.13,126.05,124.42\left(\mathrm{q}, J_{\mathrm{CF}}=3.8\right.$ $\mathrm{Hz}), 124.40\left(\mathrm{q}, J_{\mathrm{CF}}=270.9 \mathrm{~Hz}\right), 123.8\left(\mathrm{q}, J_{\mathrm{CF}}=270.9 \mathrm{~Hz}\right), 122.3,111.8$ [carbon attached to boron was not observed due to quadrupole broadening caused by the boron nucleus]. ${ }^{11} \mathrm{~B}$ NMR (193 MHz, $\left.\mathrm{C}_{6} \mathrm{D}_{6} / \mathrm{BF}_{3} \cdot \mathrm{OEt}_{2}\right) \delta$ 33.3. LRMS (EI) $m / z: 482\left(\mathrm{M}^{+}\right)$. HRMS (EI-EB) $m / z:\left(\mathrm{M}^{+}\right)$Calcd. for $\mathrm{C}_{26} \mathrm{H}_{17} \mathrm{BF}_{6} \mathrm{~N}_{2}$ : 482.1389, found: 482.1388. IR (neat): 729, 841, 1018, 1106, 1317, 1451, 1603, 3052, $3075 \mathrm{~cm}^{-1}$.<smiles>CB1c2cccc(C)c2N(C)N1c1ccccc1C</smiles>

1,3,7-Trimethyl-2-(o-tolyl)-2,3-dihydro-1H-benzo[ $d][1,2,3]$ diazaborole (4ba). According to the general procedure analogous to that described for 4aa, except that the reaction was conducted with THF $(0.5 \mathrm{~mL})$ at $-10{ }^{\circ} \mathrm{C}$ and that the crude material was purified by passing through a short pad of activated alumina (hexane: $\left.\mathrm{CH}_{2} \mathrm{Cl}_{2}=10: 1\right)$, 4 ba $(45.2 \mathrm{mg}, 0.181 \mathrm{mmol}, 90 \%$ ) was obtained from $\mathbf{2 b}$ (76.3 mg, $0.201 \mathrm{mmol})$ as a red oil. ${ }^{1} \mathrm{H}$ NMR (400 MHz, $\left.\mathrm{CDCl}_{3}\right) \delta 7.67(\mathrm{~d}, J=7.3 \mathrm{~Hz}, 1 \mathrm{H}), 7.32-7.20$ $(\mathrm{m}, 5 \mathrm{H}), 7.09$ (t, $J=7.3 \mathrm{~Hz}, 1 \mathrm{H}), 2.86(\mathrm{~s}, 3 \mathrm{H}), 2.50(\mathrm{~s}, 3 \mathrm{H}), 2.22(\mathrm{~s}, 3 \mathrm{H}), 0.59(\mathrm{~s}, 3 \mathrm{H}) .{ }^{13} \mathrm{C}$ NMR $(100$ $\left.\mathrm{MHz}, \mathrm{C}_{6} \mathrm{D}_{6}\right) \delta 157.0,140.0,136.2,132.2,131.1,129.2,129.1,127.1,126.5,122.6,122.4,38.8,19.5$, 
18.1 [carbon attached to boron was not observed due to quadrupole broadening caused by the boron nucleus]. ${ }^{11} \mathrm{~B} \mathrm{NMR}\left(193 \mathrm{MHz}, \mathrm{CDCl}_{3} / \mathrm{BF}_{3} \cdot \mathrm{OEt}_{2}\right) \delta$ 36.0. LRMS (EI) $m / z: 250\left(\mathrm{M}^{+}\right)$. HRMS (EI-TOF) $m / z:\left(\mathrm{M}^{+}\right)$Calcd. for $\mathrm{C}_{14} \mathrm{H}_{15} \mathrm{BN}_{2}: 250.1641$, found: 250.1638 . IR (neat): 720, 753, 773, 909, 1117, 1328, $1493,1597,2871,2961,3041 \mathrm{~cm}^{-1}$.<smiles>CB1c2cc(C)ccc2N(C)N1c1ccc(C)cc1</smiles>

1,3,5-Trimethyl-2-(p-tolyl)-2,3-dihydro-1H-benzo[d][1,2,3]diazaborole (4ca). According to the general procedure analogous to that described for 4aa, except that the crude material was purified by passing through a short pad of silica gel $\left(\mathrm{CH}_{2} \mathrm{Cl}_{2}\right), 4 \mathbf{c a}(42.2 \mathrm{mg}, 0.169 \mathrm{mmol}, 84 \%)$ was obtained from 2d $(76.3 \mathrm{mg}, 0.201 \mathrm{mmol})$ as a yellow solid: $\mathrm{Mp} 75-77{ }^{\circ} \mathrm{C}\left(\mathrm{CH}_{2} \mathrm{Cl}_{2} /\right.$ hexane $) .{ }^{1} \mathrm{H}$ NMR (400 $\mathrm{MHz}$, $\left.\mathrm{C}_{6} \mathrm{D}_{6}\right) \delta 7.70(\mathrm{~s}, 1 \mathrm{H}), 7.26-7.18(\mathrm{~m}, 3 \mathrm{H}), 7.02-6.95(\mathrm{~m}, 3 \mathrm{H}), 2.74(\mathrm{~s}, 3 \mathrm{H}), 2.34(\mathrm{~s}, 3 \mathrm{H}), 2.12(\mathrm{~s}, 3 \mathrm{H})$, 0.96 (s, 3H). ${ }^{13} \mathrm{C}$ NMR $\left(100 \mathrm{MHz}, \mathrm{CDCl}_{3}\right) \delta 154.9,138.7,134.4,131.0,130.7,129.9,129.4,124.5$, 111.9, 39.6, 21.0, 20.9 [carbon attached to boron was not observed due to quadrupole broadening caused by the boron nucleus]. ${ }^{11} \mathrm{~B} \mathrm{NMR}\left(193 \mathrm{MHz}, \mathrm{CDCl}_{3} / \mathrm{BF}_{3} \cdot \mathrm{OEt}_{2}\right) \delta 36.3$. LRMS (EI) $m / z: 250$ $\left(\mathrm{M}^{+}\right)$. HRMS (EI-TOF) $\mathrm{m} / z$ : $\left(\mathrm{M}^{+}\right)$Calcd. for $\mathrm{C}_{16} \mathrm{H}_{19} \mathrm{BN}_{2}: 250.1641$, found: 250.1640 . IR (neat): 700, $772,821,866,1111,1294,1329,1511,1611,2918,3004 \mathrm{~cm}^{-1}$.<smiles>Cc1ccc(N2B(c3ccccc3)c3cc(C)ccc3N2c2ccccc2)cc1</smiles>

5-Methyl-1,3-diphenyl-2-(p-tolyl)-2,3-dihydro-1 $H$-benzo $[d][1,2,3]$ diazaborole $(4 \mathrm{cc})$. According to the general procedure analogous to that described for $\mathbf{4 a a}$, except that the reaction was conducted with 3c solution $(0.250 \mathrm{~mL}, 2.0 \mathrm{M}$ in THF, $0.5 \mathrm{mmol})$ and that the crude material was purified by passing through a short pad of silica gel (hexane: $\left.\mathrm{CH}_{2} \mathrm{Cl}_{2}=10: 1\right)$, 4cc $(57.7 \mathrm{mg}, 0.154 \mathrm{mmol}$, 74\%) was obtained from $2 \mathrm{c}(78.8 \mathrm{mg}, 0.207 \mathrm{mmol})$ as a colorless solid: $\mathrm{Mp} 160-162{ }^{\circ} \mathrm{C}\left(\mathrm{Et}_{2} \mathrm{O} /\right.$ hexane $) .{ }^{1} \mathrm{H} \mathrm{NMR}$ $\left(400 \mathrm{MHz}, \mathrm{CDCl}_{3}\right) \delta 7.75(\mathrm{~s}, 1 \mathrm{H}), 7.60-7.53(\mathrm{~m}, 2 \mathrm{H}), 7.38-7.30(\mathrm{~m}, 3 \mathrm{H}), 7.30-7.06(\mathrm{~m}, 8 \mathrm{H}), 7.03(\mathrm{~d}$, $J=8.3 \mathrm{~Hz}, 1 \mathrm{H}), 6.95(\mathrm{~d}, J=8.3 \mathrm{~Hz}, 2 \mathrm{H}), 2.41(\mathrm{~s}, 3 \mathrm{H}), 2.23(\mathrm{~s}, 3 \mathrm{H}) .{ }^{13} \mathrm{C} \mathrm{NMR}\left(100 \mathrm{MHz}, \mathrm{CDCl}_{3}\right) \delta$ $153.5,143.5,138.0,135.0,134.1,131.3,131.02,131.01,130.5,129.1,128.9,128.3,127.6,126.8$, 126.2, 111.9, 21.0, 20.9 [carbon attached to boron was not observed due to quadrupole broadening caused by the boron nucleus]. ${ }^{11} \mathrm{~B}$ NMR $\left(193 \mathrm{MHz}, \mathrm{CDCl}_{3} / \mathrm{BF}_{3} \cdot \mathrm{OEt}_{2}\right) \delta 33.8$. LRMS (EI) $m / z: 374$ 
$\left(\mathrm{M}^{+}\right)$. HRMS (EI-TOF) $m / z$ : $\left(\mathrm{M}^{+}\right)$Calcd. for $\mathrm{C}_{26} \mathrm{H}_{23} \mathrm{BN}_{2}$ : 374.1954, found: 374.1962. IR (neat): 700, $730,803,829,1289,1455,1466,1595,2926,3034 \mathrm{~cm}^{-1}$.<smiles>CB1c2cc(C(C)(C)C)ccc2N(C)N1c1ccc(C(C)(C)C)cc1</smiles>

5-(tert-Butyl)-2-(4-(tert-butyl)phenyl)-1,3-dimethyl-2,3-dihydro-1 $H$-benzo[d] [1,2,3]diazaborole (4da). According to the general procedure analogous to that described for 4aa, except that the crude material was purified by passing through a short pad of silica gel (hexane: $\mathrm{CH}_{2} \mathrm{Cl}_{2}=3: 1$ ), 4 da (61.1 $\mathrm{mg}, 0.183 \mathrm{mmol}, 90 \%)$ was obtained from $2 \mathbf{d}(94.2 \mathrm{mg}, 0.204 \mathrm{mmol})$ as a yellow solid: Mp 125-127 ${ }^{\circ} \mathrm{C}\left(\mathrm{Et}_{2} \mathrm{O} /\right.$ hexane). ${ }^{1} \mathrm{H}$ NMR $\left(400 \mathrm{MHz}, \mathrm{CDCl}_{3}\right) \delta 7.80(\mathrm{~s}, 1 \mathrm{H}), 7.54(\mathrm{~d}, J=8.3 \mathrm{~Hz}, 1 \mathrm{H}), 7.40(\mathrm{~d}, J=$ $8.3 \mathrm{~Hz}, 2 \mathrm{H}), 7.21(\mathrm{~d}, J=7.8 \mathrm{~Hz}, 2 \mathrm{H}), 7.16(\mathrm{~d}, J=8.8 \mathrm{~Hz}, 1 \mathrm{H}), 2.98(\mathrm{~s}, 3 \mathrm{H}), 1.38(\mathrm{~s}, 9 \mathrm{H}), 1.35(\mathrm{~s}, 9 \mathrm{H})$, $0.83(\mathrm{~s}, 3 \mathrm{H}) .{ }^{13} \mathrm{C} \mathrm{NMR}\left(100 \mathrm{MHz}, \mathrm{C}_{6} \mathrm{D}_{6}\right) \delta 155.7,147.6,143.7,139.4,128.0,127.2,126.0,124.6$, $112.3,39.6,34.5,34.4,32.0,31.5$ [carbon attached to boron was not observed due to quadrupole broadening caused by the boron nucleus]. ${ }^{11} \mathrm{~B} \mathrm{NMR}\left(193 \mathrm{MHz}, \mathrm{CDCl}_{3} / \mathrm{BF}_{3} \cdot \mathrm{OEt}_{2}\right) \delta 36.5$. LRMS (EI) $m / z: 334\left(\mathrm{M}^{+}\right)$. HRMS (EI-TOF) $m / z$ : $\left(\mathrm{M}^{+}\right)$Calcd. for $\mathrm{C}_{22} \mathrm{H}_{31} \mathrm{BN}_{2}: 334.2580$, found: 334.2581. IR (neat): $754,818,838,1112,1298,1362,1479,1612,2865,2956 \mathrm{~cm}^{-1}$.<smiles>CC(C)(C)c1ccc(N2B(c3ccccc3)c3cc(C(C)(C)C)ccc3N2c2ccccc2)cc1</smiles>

\section{5-(tert-Butyl)-2-(4-(tert-butyl)phenyl)-1,3-diphenyl-2,3-dihydro-1H-benzo[d] [1,2,3]diazaborole}

(4dc). According to the general procedure analogous to that described for 4aa, except that the reaction was conducted with $3 \mathbf{c}$ solution $(0.250 \mathrm{~mL}, 2.0 \mathrm{M}$ in THF, $0.5 \mathrm{mmol})$ and that the crude material was purified by passing through a short pad of silica gel (hexane: $\left.\mathrm{CH}_{2} \mathrm{Cl}_{2}=10: 1\right)$, 4dc $(73.6 \mathrm{mg}, 0.161$ mmol, $81 \%)$ was obtained from $2 \mathbf{2 d}(92.4 \mathrm{mg}, 0.199 \mathrm{mmol})$ as a colorless solid: $\mathrm{Mp} 167-168{ }^{\circ} \mathrm{C}$ (Et $2 \mathrm{O} /$ hexane). ${ }^{1} \mathrm{H}$ NMR (400 MHz, $\left.\mathrm{CDCl}_{3}\right) \delta 7.93(\mathrm{~s}, 3 \mathrm{H}), 7.58-7.54(\mathrm{~m}, 2 \mathrm{H}), 7.48$ (dd, $J=8.8,1.0$ $\mathrm{Hz}, 2 \mathrm{H}), 7.38-7.33(\mathrm{~m}, 3 \mathrm{H}), 7.30-7.19(\mathrm{~m}, 4 \mathrm{H}), 7.16-7.08(\mathrm{~m}, 6 \mathrm{H}), 1.36(\mathrm{~s}, 9 \mathrm{H}), 1.22(\mathrm{~s}, 9 \mathrm{H}) .{ }^{13} \mathrm{C}$ NMR (100 MHz, $\left.\mathrm{CDCl}_{3}\right) \delta 153.0,148.2,144.0,143.3,137.8,134.1,128.8,128.2,128.0,127.6,126.9$, $126.6,126.0,125.7,125.2,111.5,34.5,34.3,31.8,31.3$ [carbon attached to boron was not observed due to quadrupole broadening caused by the boron nucleus]. ${ }^{11} \mathrm{~B} \mathrm{NMR}\left(193 \mathrm{MHz}, \mathrm{CDCl}_{3} / \mathrm{BF}_{3} \cdot \mathrm{OEt}_{2}\right.$ ) $\delta$ 34.2. LRMS (EI) $m / z$ : 458( $\left.\mathrm{M}^{+}\right)$. HRMS (EI-TOF) $m / z$ : $\left(\mathrm{M}^{+}\right.$) Calcd. for $\mathrm{C}_{32} \mathrm{H}_{35} \mathrm{BN}_{2}: 458.2893$, found: 458.2875. IR (neat): 700, 744, 814, 839, 1204, 1364, 1470, 1512, 1593, $2956 \mathrm{~cm}^{-1}$. 
<smiles>CB1c2c(C)cc(C)cc2N(C)N1c1cc(C)cc(C)c1</smiles>

2-(3,5-Dimethylphenyl)-1,3,4,6-tetramethyl-2,3-dihydro-1 $H$-benzo[d] $[1,2,3]$ diazaborole (4ea). According to the general procedure analogous to that described for 4aa, except that the crude material was purified by passing through a short pad of silica gel (hexane: $\left.\mathrm{CH}_{2} \mathrm{Cl}_{2}=10: 1\right)$, 4ea (51.0 mg, 0.183 mmol, 91\%) was obtained from $2 \mathbf{e}(82.1 \mathrm{mg}, 0.201 \mathrm{mmol})$ as a red oil. ${ }^{1} \mathrm{H}$ NMR $\left(400 \mathrm{MHz}, \mathrm{CDCl}_{3}\right) \delta$ $6.90(\mathrm{~s}, 2 \mathrm{H}), 6.84(\mathrm{~s}, 1 \mathrm{H}), 6.83(\mathrm{~s}, 1 \mathrm{H}), 6.70(\mathrm{~s}, 1 \mathrm{H}), 2.96(\mathrm{~s}, 3 \mathrm{H}), 2.56(\mathrm{~s}, 3 \mathrm{H}), 2.41(\mathrm{~s}, 3 \mathrm{H}), 2.35(\mathrm{~s}$, $6 \mathrm{H}), 0.86(\mathrm{~s}, 3 \mathrm{H}) .{ }^{13} \mathrm{C} \mathrm{NMR}\left(100 \mathrm{MHz}, \mathrm{C}_{6} \mathrm{D}_{6}\right) \delta 158.2,142.6,141.7,139.9,138.5,127.0,123.9,123.5$, $110.2,39.2,22.1,21.4,21.3$ [carbon attached to boron was not observed due to quadrupole broadening caused by the boron nucleus]. ${ }^{11} \mathrm{~B}$ NMR $\left(193 \mathrm{MHz}, \mathrm{CDCl}_{3} / \mathrm{BF}_{3} \cdot \mathrm{OEt}_{2}\right) \delta$ 35.9. LRMS (EI) $m / z: 278$ $\left(\mathrm{M}^{+}\right)$. HRMS (EI-TOF) $m / z:\left(\mathrm{M}^{+}\right)$Calcd. for $\mathrm{C}_{18} \mathrm{H}_{23} \mathrm{BN}_{2}: 278.1954$, found: 278.1975. IR (neat): 742 , $827,841,1111,1322,1441,1595,1607,2916 \mathrm{~cm}^{-1}$.<smiles>CB1c2cc(F)ccc2N(C)N1c1ccc(F)cc1</smiles>

\section{5-Fluoro-2-(4-fluorophenyl)-1,3-dimethyl-2,3-dihydro-1 $H$-benzo $[d][1,2,3]$ diazaborole}

(4fa).

According to the general procedure analogous to that described for 4aa, except that the crude material was purified by passing through a short pad of activated alumina (hexane: $\mathrm{CH}_{2} \mathrm{Cl}_{2}=1: 1$ ), $\mathbf{4 f a}(48.7 \mathrm{mg}$, $0.189 \mathrm{mmol}, 94 \%)$ was obtained from $2 \mathrm{f}(77.9 \mathrm{mg}, 0.201 \mathrm{mmol})$ as a yellow solid: $\mathrm{Mp} 85-87^{\circ} \mathrm{C}$ (Et $2 \mathrm{O} /$ hexane). ${ }^{1} \mathrm{H}$ NMR (400 MHz, $\left.\mathrm{C}_{6} \mathrm{D}_{6}\right) \delta 7.48(\mathrm{dd}, 8.1,2.7 \mathrm{~Hz}, 1 \mathrm{H}), 7.08(\mathrm{dt}, 2.4,8.8 \mathrm{~Hz}, 1 \mathrm{H})$, 6.93-6.86 (m, 2H), 6.80-6.68 (m, 3H), 2.47 (s, 3H), $0.69(\mathrm{~s}, 3 \mathrm{H}) .{ }^{13} \mathrm{C} \mathrm{NMR}\left(150 \mathrm{MHz}, \mathrm{CDCl}_{3}\right) \delta 160.3$ $\left(\mathrm{d}, J_{\mathrm{CF}}=242.7 \mathrm{~Hz}\right), 158.3\left(\mathrm{~d}, J_{\mathrm{CF}}=238.4 \mathrm{~Hz}\right), 152.9,136.9\left(\mathrm{~d}, J_{\mathrm{CF}}=2.9 \mathrm{~Hz}\right), 126.2\left(\mathrm{~d}, J_{\mathrm{CF}}=8.6 \mathrm{~Hz}\right)$, $117.8\left(\mathrm{~d}, J_{\mathrm{CF}}=25.8 \mathrm{~Hz}\right), 115.7\left(\mathrm{~d}, J_{\mathrm{CF}}=23.0 \mathrm{~Hz}\right), 115.4\left(\mathrm{~d}, J_{\mathrm{CF}}=20.1 \mathrm{~Hz}\right), 113.4\left(\mathrm{~d}, J_{\mathrm{CF}}=8.6 \mathrm{~Hz}\right)$, 39.7 [carbon attached to boron was not observed due to quadrupole broadening caused by the boron nucleus]. ${ }^{11} \mathrm{~B}$ NMR (193 MHz, $\left.\mathrm{CDCl}_{3} / \mathrm{BF}_{3} \cdot \mathrm{OEt}_{2}\right) \delta 36.3$. LRMS (EI) $m / z: 258\left(\mathrm{M}^{+}\right)$. HRMS (EI-TOF) $m / z$ : $\left(\mathrm{M}^{+}\right)$Calcd. for $\mathrm{C}_{14} \mathrm{H}_{13} \mathrm{BF}_{2} \mathrm{~N}_{2}$ : 258.1140, found: 258.1157. IR (neat): 775, 813,837, 1188, 1215, $1263,1505,2872,2930 \mathrm{~cm}^{-1}$. 
<smiles>Fc1ccc(N2B(c3ccccc3)c3cc(F)ccc3N2c2ccccc2)cc1</smiles>

\section{5-Fluoro-2-(4-fluorophenyl)-1,3-diphenyl-2,3-dihydro-1 $H$-benzo $[d][1,2,3]$ diazaborole}

(4fe).

According to the general procedure analogous to that described for $\mathbf{4 a a}$, except that the reaction was conducted with $3 \mathbf{c}$ solution $(0.250 \mathrm{~mL}, 2.0 \mathrm{M}$ in THF, $0.5 \mathrm{mmol})$ and that the crude material was purified by passing through a short pad of activated alumina (hexane: $\left.\mathrm{CH}_{2} \mathrm{Cl}_{2}=1: 1\right), \mathbf{4 f c}(62.1 \mathrm{mg}$, $0.162 \mathrm{mmol}, 81 \%)$ was obtained from $2 \mathbf{f}(77.6 \mathrm{mg}, 0.200 \mathrm{mmol})$ as a colorless solid: $\mathrm{Mp} 130-132{ }^{\circ} \mathrm{C}$ $\left(\mathrm{CH}_{2} \mathrm{Cl}_{2} /\right.$ hexane). ${ }^{1} \mathrm{H}$ NMR $\left(400 \mathrm{MHz}, \mathrm{C}_{6} \mathrm{D}_{6}\right) \delta 7.78(\mathrm{dd}, J=8.3,2.4 \mathrm{~Hz}, 1 \mathrm{H}), 7.63-7.57(\mathrm{~m}, 2 \mathrm{H})$, $7.28-7.18(\mathrm{~m}, 3 \mathrm{H}), 7.00(\mathrm{dt}, J=2.6,8.9 \mathrm{~Hz}, 1 \mathrm{H}), 6.92-6.76(\mathrm{~m}, 8 \mathrm{H}), 6.46(\mathrm{t}, J=8.8 \mathrm{~Hz}, 2 \mathrm{H}) .{ }^{13} \mathrm{C}$ NMR $\left(100 \mathrm{MHz}, \mathrm{CDCl}_{3}\right) \delta 160.6\left(\mathrm{~d}, J_{\mathrm{CF}}=250.3 \mathrm{~Hz}\right), 158.7\left(\mathrm{~d}, J_{\mathrm{CF}}=237.1 \mathrm{~Hz}\right), 151.6,142.9,136.4$ $\left(\mathrm{d}, J_{\mathrm{CF}}=4.1 \mathrm{~Hz}\right), 133.9,129.2,128.8,128.1\left(\mathrm{~d}, J_{\mathrm{CF}}=8.2 \mathrm{~Hz}\right), 127.9,127.5,126.4,118.3\left(\mathrm{~d}, J_{\mathrm{CF}}=24.7\right.$ $\mathrm{Hz}), 115.6\left(\mathrm{~d}, J_{\mathrm{CF}}=20.6 \mathrm{~Hz}\right), 115.4\left(\mathrm{~d}, J_{\mathrm{CF}}=23.1 \mathrm{~Hz}\right), 113.6\left(\mathrm{~d}, J_{\mathrm{CF}}=8.2 \mathrm{~Hz}\right)$ [carbon attached to boron was not observed due to quadrupole broadening caused by the boron nucleus]. ${ }^{11} \mathrm{~B}$ NMR (193 $\left.\mathrm{MHz}, \mathrm{C}_{6} \mathrm{D}_{6} / \mathrm{BF}_{3} \cdot \mathrm{OEt}_{2}\right) \delta$ 33.1. LRMS (EI) $\mathrm{m} / z$ : $382\left(\mathrm{M}^{+}\right)$. HRMS (EI-TOF) $\mathrm{m} / z$ : $\left(\mathrm{M}^{+}\right.$) Calcd. for $\mathrm{C}_{24} \mathrm{H}_{17} \mathrm{BF}_{2} \mathrm{~N}_{2}$ : 382.1453, found: 382.1429. IR (neat): 805 844, 1088, 1224, 1466, 1506, 1594, 3060, $3355 \mathrm{~cm}^{-1}$.<smiles>CN1c2ccc(Cl)cc2N(C)N1c1ccc(Cl)cc1</smiles>

\section{5-Chloro-2-(4-chlorophenyl)-1,3-dimethyl-2,3-dihydro-1 $H$-benzo $[d][1,2,3]$ diazaborole}

According to the general procedure analogous to that described for 4aa, except that the crude material was purified by passing through a short pad of silica gel (hexane: $\left.\mathrm{CH}_{2} \mathrm{Cl}_{2}=20: 1\right)$, 4ga (53.1 mg, 0.182 mmol, 91\%) was obtained from $2 \mathrm{~g}(84.4 \mathrm{mg}, 0.201 \mathrm{mmol})$ as a yellow solid: $\mathrm{Mp} 100-102{ }^{\circ} \mathrm{C}$ $\left(\mathrm{CH}_{2} \mathrm{Cl}_{2} /\right.$ hexane). ${ }^{1} \mathrm{H}$ NMR $\left(400 \mathrm{MHz}, \mathrm{C}_{6} \mathrm{D}_{6}\right) \delta 7.81(\mathrm{~d}, J=2.4 \mathrm{~Hz}, 1 \mathrm{H}), 7.33(\mathrm{dd}, J=8.8,2.4 \mathrm{~Hz}, 1 \mathrm{H})$, $7.08-7.02(\mathrm{~m}, 2 \mathrm{H}), 6.83-6.77(\mathrm{~m}, 2 \mathrm{H}), 6.65(\mathrm{~d}, J=8.8 \mathrm{~Hz}, 1 \mathrm{H}), 2.42(\mathrm{~s}, 3 \mathrm{H}), 0.66(\mathrm{~s}, 3 \mathrm{H}) .{ }^{13} \mathrm{C}$ NMR $\left(100 \mathrm{MHz}, \mathrm{CDCl}_{3}\right) \delta 154.7,139.4,130.6,130.3,130.1,129.1,126.5,125.7,113.4,39.3$ [carbon attached to boron was not observed due to quadrupole broadening caused by the boron nucleus]. ${ }^{11} \mathrm{~B}$ NMR (193 MHz, $\left.\mathrm{CDCl}_{3} / \mathrm{BF}_{3} \cdot \mathrm{OEt}_{2}\right) \delta$ 36.41. LRMS (EI) $\mathrm{m} / z: 290\left(\mathrm{M}^{+}\right)$. HRMS (EI-TOF) $m / z:\left(\mathrm{M}^{+}\right)$ Calcd. for $\mathrm{C}_{14} \mathrm{H}_{13} \mathrm{BCl}_{2} \mathrm{~N}_{2}$ : 290.0549, found: 290.0539. IR (neat): 722, 809, 834, 1088, 1292, 1446, $1489,1599,2868,2978 \mathrm{~cm}^{-1}$. 
<smiles>Clc1ccc(N2B(c3ccccc3)c3cc(Cl)ccc3N2c2ccccc2)cc1</smiles>

5-Chloro-2-(4-chlorophenyl)-1,3-diphenyl-2,3-dihydro-1H-benzo $[d][1,2,3]$ diazaborole

(4gc).

According to the general procedure analogous to that described for $\mathbf{4 a a}$, except that the reaction was conducted with $3 \mathbf{c}$ solution $(0.250 \mathrm{~mL}, 2.0 \mathrm{M}$ in THF, $0.5 \mathrm{mmol})$ and that the crude material was purified by passing through a short pad of silica gel (hexane: $\left.\mathrm{CH}_{2} \mathrm{Cl}_{2}=10: 1\right), 4$ gc $(71.6 \mathrm{mg}, 0.172$ mmol, 84\%) was obtained from $2 \mathrm{~g}(86.4 \mathrm{mg}, 0.205 \mathrm{mmol})$ as a colorless solid: $\mathrm{Mp} 152-154{ }^{\circ} \mathrm{C}$ (Et ${ }_{2} \mathrm{O} /$ hexane). ${ }^{1} \mathrm{H}$ NMR (400 MHz, $\left.\mathrm{C}_{6} \mathrm{D}_{6}\right) \delta 8.12(\mathrm{~d}, J=2.4 \mathrm{~Hz}, 1 \mathrm{H}), 7.58-7.53(\mathrm{~m}, 2 \mathrm{H}), 7.28-7.19$ $(\mathrm{m}, 4 \mathrm{H}), 6.90-6.75(\mathrm{~m}, 10 \mathrm{H}) .{ }^{13} \mathrm{C} \mathrm{NMR}\left(150 \mathrm{MHz}, \mathrm{CDCl}_{3}\right) \delta 153.3,142.3,138.7,133.9,131.2,130.6$, $130.3,129.2,128.9,128.7,127.9,127.6,127.4,127.1,126.2,113.5$. [carbon attached to boron was not observed due to quadrupole broadening caused by the boron nucleus]. ${ }^{11} \mathrm{~B} \mathrm{NMR}$ (193 $\mathrm{MHz}$, $\left.\mathrm{CDCl}_{3} / \mathrm{BF}_{3} \cdot \mathrm{OEt}_{2}\right) \delta$ 33.8. LRMS (EI) $m / z: 414\left(\mathrm{M}^{+}\right)$. HRMS (EI-TOF) $\mathrm{m} / z:\left(\mathrm{M}^{+}\right)$Calcd. for $\mathrm{C}_{24} \mathrm{H}_{17} \mathrm{BCl}_{2} \mathrm{~N}_{2}$ : 414.0862, found: 414.0869. IR (neat): 764, 803, 833, 1090, 1280, 1446, 1491, 1598, $3044,3074 \mathrm{~cm}^{-1}$.<smiles>CB1c2cc(Br)ccc2N(C)N1c1ccc(Br)cc1</smiles>

5-Bromo-2-(4-bromophenyl)-1,3-dimethyl-2,3-dihydro-1H-benzo $[d][1,2,3]$ diazaborole

(4ha).

According to the general procedure analogous to that described for 4aa, except that the crude material was purified by passing through a short pad of silica gel (hexane: $\left.\mathrm{CH}_{2} \mathrm{Cl}_{2}=3: 1\right)$, 4ha $(66.1 \mathrm{mg}, 0.174$ mmol, 89\%) was obtained from $2 \mathbf{h}(99.8 \mathrm{mg}, 0.196 \mathrm{mmol})$ as a yellow solid: $\mathrm{Mp} 122-123{ }^{\circ} \mathrm{C}$ $\left(\mathrm{CH}_{2} \mathrm{Cl}_{2} /\right.$ hexane). ${ }^{1} \mathrm{H}$ NMR $\left(400 \mathrm{MHz}, \mathrm{CDCl}_{3}\right) \delta 7.87(\mathrm{~d}, J=1.9 \mathrm{~Hz}, 1 \mathrm{H}), 7.55-7.50(\mathrm{~m}, 3 \mathrm{H})$, 7.18-7.12 (m, 2H), $7.07(\mathrm{~d}, J=8.8 \mathrm{~Hz}, 1 \mathrm{H}), 2.97$ (s, 3H), $0.78(\mathrm{~s}, 3 \mathrm{H}) .{ }^{13} \mathrm{C} \mathrm{NMR}\left(100 \mathrm{MHz}, \mathrm{CDCl}_{3}\right)$ $\delta 155.0,139.8,133.5,132.8,132.0,126.1,118.3,114.2,113.9,39.2$ [carbon attached to boron was not observed due to quadrupole broadening caused by the boron nucleus]. ${ }^{11} \mathrm{~B} \mathrm{NMR}$ (193 $\mathrm{MHz}$, $\left.\mathrm{CDCl}_{3} / \mathrm{BF}_{3} \cdot \mathrm{OEt}_{2}\right) \delta$ 36.4. LRMS (EI) $\mathrm{m} / z: 380\left(\mathrm{M}^{+}\right)$. HRMS (EI-TOF) $\mathrm{m} / z:\left(\mathrm{M}^{+}\right)$Calcd. for $\mathrm{C}_{14} \mathrm{H}_{13} \mathrm{BBr}_{2} \mathrm{~N}_{2}$ : 377.9539, found: 377.9532. IR (neat): 801, 836, 1006, 1100, 1256, 1293, 1485, 1587, $1596,2896 \mathrm{~cm}^{-1}$. 
<smiles>Brc1ccc(N2B(c3ccccc3)c3cc(Br)ccc3N2c2ccccc2)cc1</smiles>

5-Bromo-2-(4-bromophenyl)-1,3-diphenyl-2,3-dihydro-1 $H$-benzo[d][1,2,3]diazaborole

(4hc).

According to the general procedure analogous to that described for $\mathbf{4 a a}$, except that the reaction was conducted with 3c solution $(0.250 \mathrm{~mL}, 2.0 \mathrm{M}$ in THF, $0.500 \mathrm{mmol})$ and $\mathrm{CH}_{2} \mathrm{Cl}_{2}(1.0 \mathrm{~mL})$ at $-20{ }^{\circ} \mathrm{C}$ and that the crude material was purified by passing through a short pad of silica gel (hexane: $\mathrm{CH}_{2} \mathrm{Cl}_{2}=$ 1:1), 4hc (72.7 $\mathrm{mg}, 0.144 \mathrm{mmol}, 72 \%)$ was obtained from $\mathbf{2 h}(101.8 \mathrm{mg}, 0.200 \mathrm{mmol})$ as a colorless solid: Mp 196-197 ${ }^{\circ} \mathrm{C}\left(\mathrm{CH}_{2} \mathrm{Cl}_{2} /\right.$ hexane). ${ }^{1} \mathrm{H} \mathrm{NMR}\left(400 \mathrm{MHz}, \mathrm{CDCl}_{3}\right) \delta 8.04(\mathrm{~d}, J=2.0 \mathrm{~Hz}, 1 \mathrm{H})$, 7.53-7.45 (m, 3H), 7.42-7.33 (m, 3H), 7.33-7.26 (m, 4H), 7.23-7.13 (m, 3H), 7.10-7.05 (m, 2H), $6.97(\mathrm{~d}, J=8.8 \mathrm{~Hz}, 1 \mathrm{H}) .{ }^{13} \mathrm{C} \mathrm{NMR}\left(100 \mathrm{MHz}, \mathrm{CDCl}_{3}\right) \delta 153.6,142.2,139.1,133.9,133.8,133.0$, $131.7,129.3,128.9,128.0,127.7,127.6,126.2,119.2,114.9,114.0$ [carbon attached to boron was not observed due to quadrupole broadening caused by the boron nucleus]. ${ }^{11} \mathrm{~B}$ NMR (193 MHz, $\left.\mathrm{CDCl}_{3} / \mathrm{BF}_{3} \cdot \mathrm{OEt}_{2}\right) \delta$ 33.9. LRMS (EI) $m / z: 503\left(\mathrm{M}^{+}\right)$. HRMS (EI-TOF) $m / z:\left(\mathrm{M}^{+}\right)$Calcd. for $\mathrm{C}_{24} \mathrm{H}_{17} \mathrm{BBr}_{2} \mathrm{~N}_{2}: 501.9852$, found: 501.9848. IR (neat): 712, 759, 830, 897, 1070, 1280, 1444, 1490, $1588,3045,3074 \mathrm{~cm}^{-1}$.<smiles>CB1c2cc(I)ccc2N(c2ccc(I)cc2)N1C</smiles>

5-Iodo-2-(4-iodophenyl)-1,3-dimethyl-2,3-dihydro-1H-benzo $[d][1,2,3]$ diazaborole

(4ia).

According to the general procedure analogous to that described for $4 \mathbf{a a}$, except that the reaction was conducted with $\mathrm{CH}_{2} \mathrm{Cl}_{2}(2.0 \mathrm{~mL})$ and that the crude material was purified by passing through a short pad of activated alumina (hexane: $\left.\mathrm{CH}_{2} \mathrm{Cl}_{2}=1: 1\right), 4 \mathbf{i a}(87.7 \mathrm{mg}, 0.185 \mathrm{mmol}, 93 \%)$ was obtained from $2 \mathbf{i}$ (119.6 mg, $0.198 \mathrm{mmol})$ as a yellow solid: $\mathrm{Mp} 119-121{ }^{\circ} \mathrm{C}\left(\mathrm{CH}_{2} \mathrm{Cl}_{2} / \mathrm{hexane}\right) .{ }^{1} \mathrm{H} \mathrm{NMR}(400 \mathrm{MHz}$, $\left.\mathrm{C}_{6} \mathrm{D}_{6}\right) \delta 8.18(\mathrm{~d}, J=1.5 \mathrm{~Hz}, 1 \mathrm{H}), 7.63(\mathrm{dd}, J=8.5,1.7 \mathrm{~Hz}, 1 \mathrm{H}), 7.40(\mathrm{~d}, J=8.8 \mathrm{~Hz}, 2 \mathrm{H}), 6.61(\mathrm{~d}, J=$ $8.3 \mathrm{~Hz}, 2 \mathrm{H}), 6.50(\mathrm{~d}, J=8.8 \mathrm{~Hz}, 1 \mathrm{H}), 2.40(\mathrm{~s}, 3 \mathrm{H}), 0.63(\mathrm{~s}, 3 \mathrm{H}) .{ }^{13} \mathrm{C} \mathrm{NMR}\left(100 \mathrm{MHz}, \mathrm{CDCl}_{3}\right) \delta 155.4$, $140.4,139.9,138.2,138.0,126.3,114.3,89.2,84.7,39.1$ [carbon attached to boron was not observed due to quadrupole broadening caused by the boron nucleus]. ${ }^{11} \mathrm{~B}$ NMR (193 MHz, $\left.\mathrm{C}_{6} \mathrm{D}_{6} / \mathrm{BF}_{3} \cdot \mathrm{OEt}_{2}\right) \delta$ 35.2. LRMS (EI) $m / z$ : $473\left(\mathrm{M}^{+}\right)$. HRMS (EI-TOF) $m / z$ : $\left(\mathrm{M}^{+}\right)$Calcd. for $\mathrm{C}_{14} \mathrm{H}_{13} \mathrm{BI}_{2} \mathrm{~N}_{2}: 473.9261$, found: 4739236. IR (neat): 736, 808, 819, 827, 1142, 1249, 1289, 1482, 1584, 2921, $2970 \mathrm{~cm}^{-1}$. 
<smiles>Ic1ccc(N2B(c3ccccc3)c3cc(I)ccc3N2c2ccccc2)cc1</smiles>

5-Iodo-2-(4-iodophenyl)-1,3-diphenyl-2,3-dihydro-1 $H$-benzo $[d][1,2,3]$ diazaborole

(4ic).

According to the general procedure analogous to that described for $4 \mathbf{a a}$, except that the reaction was conducted with $3 \mathbf{c}$ solution $(0.250 \mathrm{~mL}, 2.0 \mathrm{M}$ in THF, $0.5 \mathrm{mmol})$ and $\mathrm{CH}_{2} \mathrm{Cl}_{2}(1.0 \mathrm{~mL})$ at $-20{ }^{\circ} \mathrm{C}$ and that the crude material was purified by passing through a short pad of activated alumina (hexane: $\mathrm{CH}_{2} \mathrm{Cl}_{2}=1: 1$ ) and by recrystallization from $\mathrm{CH}_{2} \mathrm{Cl}_{2} /$ hexane, 4 ic $(81.5 \mathrm{mg}, 0.136 \mathrm{mmol}, 68 \%$ ) was obtained from $2 \mathbf{i}(121.7 \mathrm{mg}, 0.202 \mathrm{mmol})$ as a colorless solid: $\mathrm{Mp} 219-220{ }^{\circ} \mathrm{C}\left(\mathrm{CH}_{2} \mathrm{Cl}_{2} / \mathrm{hexane}\right)$. ${ }^{1} \mathrm{H}$ NMR (400 MHz, C6 $\left.\mathrm{D}_{6}\right) \delta 8.49(\mathrm{~d}, J=1.5 \mathrm{~Hz}, 1 \mathrm{H}), 7.60-7.50(\mathrm{~m}, 3 \mathrm{H}), 7.21-7.18(\mathrm{~m}, 3 \mathrm{H})$, $7.13-7.10(\mathrm{~m}, 2 \mathrm{H}), 6.88-6.72(\mathrm{~m}, 5 \mathrm{H}), 6.66(\mathrm{~d}, J=8.8 \mathrm{~Hz}, 1 \mathrm{H}), 6.64-6.59(\mathrm{~m}, 2 \mathrm{H}) .{ }^{13} \mathrm{C}$ NMR $(150$ $\left.\mathrm{MHz}, \mathrm{C}_{6} \mathrm{D}_{6}\right) \delta 154.0,142.1,140.2,139.8,138.5,137.7,133.9,129.3,128.9,128.01,127.99,127.6$, $126.1,114.4,90.3,85.4$ [carbon attached to boron was not observed due to quadrupole broadening caused by the boron nucleus]. ${ }^{11} \mathrm{~B}$ NMR $\left(193 \mathrm{MHz}, \mathrm{CDCl}_{3} / \mathrm{BF}_{3} \cdot \mathrm{OEt}_{2}\right) \delta 33.5$. LRMS (EI) $m / z: 597$ $\left(\mathrm{M}^{+}\right)$. HRMS (EI-TOF) $m / z$ : $\left(\mathrm{M}^{+}\right)$Calcd. for $\mathrm{C}_{24} \mathrm{H}_{17} \mathrm{BI}_{2} \mathrm{~N}_{2}$ : 597.9574, found: 597.9554. IR (neat): 733, $756,827,1111,1278,1442,1484,1582,2850,2918 \mathrm{~cm}^{-1}$.<smiles>CB1c2cc(OC(F)(F)F)ccc2N(C)N1c1ccc(OC(F)(F)F)cc1</smiles>

\section{1,3-Dimethyl-5-(trifluoromethoxy)-2-(4-(trifluoromethoxy)phenyl)-2,3-dihydro-1H-}

benzo $[d][1,2,3]$ diazaborole $(4 \mathbf{j a})$. According to the general procedure analogous to that described for 4aa, except that the reaction was conducted at $-40{ }^{\circ} \mathrm{C}$ and that the crude material was purified by passing through a short pad of activated alumina (hexane: $\left.\mathrm{CH}_{2} \mathrm{Cl}_{2}=1: 1\right), 4 \mathbf{j a}(73.5 \mathrm{mg}, 0.188 \mathrm{mmol}$, 94\%) was obtained from $\mathbf{2} \mathbf{j}$ (103.8 $\mathrm{mg}, 0.200 \mathrm{mmol})$ as a yellow solid: $\mathrm{Mp} 70-74{ }^{\circ} \mathrm{C}\left(\mathrm{CH}_{2} \mathrm{Cl}_{2} / \mathrm{hexane}\right)$. ${ }^{1} \mathrm{H}$ NMR $\left(400 \mathrm{MHz}, \mathrm{CDCl}_{3}\right) \delta 7.61(\mathrm{~s}, 1 \mathrm{H}), 7.35-7.24(\mathrm{~m}, 5 \mathrm{H}), 7.20(\mathrm{~d}, J=8.8 \mathrm{~Hz}, 1 \mathrm{H}), 3.01(\mathrm{~s}, 3 \mathrm{H})$, $0.82(\mathrm{~s}, 3 \mathrm{H}) .{ }^{13} \mathrm{C} \mathrm{NMR}\left(100 \mathrm{MHz}, \mathrm{CDCl}_{3}\right) \delta 154.7,146.4,143.7,139.4,125.6,123.9,123.0,121.7$, $120.8\left(\mathrm{~d}, J_{\mathrm{CF}}=257.3 \mathrm{~Hz}\right), 120.6\left(\mathrm{q}, J_{\mathrm{CF}}=257.9 \mathrm{~Hz}\right), 113.1,39.4$ [carbon attached to boron was not observed due to quadrupole broadening caused by the boron nucleus]. ${ }^{11} \mathrm{~B} \mathrm{NMR}$ (193 $\mathrm{MHz}$, $\left.\mathrm{CDCl}_{3} / \mathrm{BF}_{3} \cdot \mathrm{OEt}_{2}\right) \quad \delta$ 36.8. LRMS (EI) $\mathrm{m} / z$ : 390 $\left(\mathrm{M}^{+}\right)$. HRMS (EI-TOF) $\mathrm{m} / \mathrm{z}:\left(\mathrm{M}^{+}\right.$) Calcd. for $\mathrm{C}_{16} \mathrm{H}_{13} \mathrm{BF}_{6} \mathrm{~N}_{2} \mathrm{O}_{2}: 390.0974$, found: 390.0975. IR (neat): 813, 819, 855, 1157, 1200, 1247, 1506, 2881, $2930 \mathrm{~cm}^{-1}$. 
<smiles>FC(F)(F)Oc1ccc(N2B(c3ccccc3)c3cc(OC(F)(F)F)ccc3N2c2ccccc2)cc1</smiles>

\section{1,3-Diphenyl-5-(trifluoromethoxy)-2-(4-(trifluoromethoxy)phenyl)-2,3-dihydro-1H-}

benzo $[d][1,2,3]$ diazaborole $(4 \mathrm{jc})$. According to the general procedure analogous to that described for 4aa, except that the reaction was conducted with $3 \mathbf{c}$ solution $(0.250 \mathrm{~mL}, 2.0 \mathrm{M}$ in THF, $0.5 \mathrm{mmol})$ and that the crude material was purified by passing through a short pad of activated alumina (hexane: $\mathrm{CH}_{2} \mathrm{Cl}_{2}=1: 1$ ), $4 \mathbf{j c}$ (70.4 mg, $0.137 \mathrm{mmol}, 69 \%$ ) was obtained from $\mathbf{2 j}$ (103.5 mg, $0.199 \mathrm{mmol}$ ) as a colorless solid: $\mathrm{Mp} 89-91{ }^{\circ} \mathrm{C}\left(\mathrm{CH}_{2} \mathrm{Cl}_{2} /\right.$ hexane). ${ }^{1} \mathrm{H}$ NMR $\left(400 \mathrm{MHz}, \mathrm{C}_{6} \mathrm{D}_{6}\right) \delta 7.97(\mathrm{~s}, 1 \mathrm{H})$, $7.58-7.51(\mathrm{~m}, 2 \mathrm{H}), 7.23-7.18(\mathrm{~m}, 3 \mathrm{H}), 7.11(\mathrm{~d}, J=10.2 \mathrm{~Hz}, 1 \mathrm{H}), 6.91-6.73(\mathrm{~m}, 8 \mathrm{H}), 6.62(\mathrm{~d}, J=8.3$ $\mathrm{Hz}, 2 \mathrm{H}) .{ }^{13} \mathrm{C} \mathrm{NMR}\left(100 \mathrm{MHz}, \mathrm{CDCl}_{3}\right) \delta 153.2,146.9,144.2,142.2,138.6,133.9,129.3,129.0,128.1$, 127.7, 127.5, 126.3, 124.2, 123.4, 121.0, $120.8\left(\mathrm{q}, J_{\mathrm{CF}}=254.4 \mathrm{~Hz}\right), 120.4\left(\mathrm{q}, J_{\mathrm{CF}}=256.0 \mathrm{~Hz}\right), 113.3$ [carbon attached to boron was not observed due to quadrupole broadening caused by the boron nucleus]. ${ }^{11} \mathrm{~B}$ NMR (193 MHz, $\left.\mathrm{C}_{6} \mathrm{D}_{6} / \mathrm{BF}_{3} \cdot \mathrm{OEt}_{2}\right) \delta 33.4$. LRMS (EI) $m / z: 514\left(\mathrm{M}^{+}\right)$. HRMS (EI-EB) $m / z$ : $\left(\mathrm{M}^{+}\right)$Calcd. for $\mathrm{C}_{26} \mathrm{H}_{17} \mathrm{BF}_{6} \mathrm{~N}_{2} \mathrm{O}_{2}: 514.1287$, found: 514.1280. IR (neat): 703, 809, 1107, 1154, 1243, $1464,1507,3079 \mathrm{~cm}^{-1}$.

Procedure of stepwise-treatment of $2 \mathrm{a}$ with $3 \mathrm{a}$ and $3 \mathrm{c}$ (Scheme 1, eq. 1).<smiles>Brc1ccccc1-n1nnc2ccccc21</smiles>

$2 \mathbf{a}$
1) $3 \mathrm{a}$ (1.0 equiv.) $\mathrm{CH}_{2} \mathrm{Cl}_{2}, 0^{\circ} \mathrm{C}, 5 \mathrm{~min}$

2) $3 c$ (1.0 equiv.) $\mathrm{CH}_{2} \mathrm{Cl}_{2}, 0{ }^{\circ} \mathrm{C}, 5 \mathrm{~min}$<smiles>CN1c2ccccc2B(c2ccccc2)N1c1ccccc1</smiles>

5

1-Methyl-2,3-diphenyl-2,3-dihydro-1 $H$-benzo $[\boldsymbol{d}][\mathbf{1 , 2 , 3}]$ diazaborole (5). To a mixture of 2 a (106.0 $\mathrm{mg}, 0.301 \mathrm{mmol})$ and $\mathrm{CH}_{2} \mathrm{Cl}_{2}(1.0 \mathrm{~mL})$ in an oven-dried vial equipped with a stirrer bar was added $3 \mathbf{a}$ solution $\left(0.105 \mathrm{~mL}, 3.0 \mathrm{M}\right.$ in $\left.\mathrm{Et}_{2} \mathrm{O}, 0.315 \mathrm{mmol}\right)$ at $0{ }^{\circ} \mathrm{C}$. After being stirred at $0{ }^{\circ} \mathrm{C}$ for $5 \mathrm{~min}, 3 \mathrm{c}$ solution $(0.165 \mathrm{~mL}, 2.0 \mathrm{M}$ in THF, $0.330 \mathrm{mmol}$ ) was added at the same temperature. After being further stirred at $0{ }^{\circ} \mathrm{C}$ for $5 \mathrm{~min}, \mathrm{H}_{2} \mathrm{O}(2 \mathrm{~mL})$ was added, and the organic materials were extracted with $\mathrm{CH}_{2} \mathrm{Cl}_{2}(10 \mathrm{~mL} \times 3)$. The combined organic layer was washed with $\mathrm{H}_{2} \mathrm{O}(10 \mathrm{~mL})$ and brine $(10 \mathrm{~mL})$, dried over $\mathrm{Na}_{2} \mathrm{SO}_{4}$, and concentrated. Yield of 5 was determined to be $94 \%$ by ${ }^{1} \mathrm{H}$ NMR spectroscopy using 1,1,2-trichloroethane as an internal standard. The crude material was purified by passing through a short pad of activated alumina $\left(\mathrm{CH}_{2} \mathrm{Cl}_{2}\right)$ to give the solid material, which was washed with cold hexane to afford $\mathbf{5}(52.8 \mathrm{mg}, 0.186 \mathrm{mmol}, 62 \%)$ as a colorless solid: $\mathrm{Mp} 113-114{ }^{\circ} \mathrm{C}\left(\mathrm{CH}_{2} \mathrm{Cl}_{2} / \mathrm{hexane}\right)$. ${ }^{1} \mathrm{H}$ NMR $\left(400 \mathrm{MHz}, \mathrm{C}_{6} \mathrm{D}_{6}\right) \delta 8.03(\mathrm{~d}, J=7.3 \mathrm{~Hz}, 1 \mathrm{H}), 7.63(\mathrm{dd}, J=7.6,1.7 \mathrm{~Hz}, 2 \mathrm{H}), 7.36(\mathrm{dt}, J=1.2$, 
$7.7 \mathrm{~Hz}, 1 \mathrm{H}), 7.23-7.05(\mathrm{~m}, 6 \mathrm{H}), 7.03-6.94(\mathrm{~m}, 3 \mathrm{H}), 6.89(\mathrm{t}, J=7.3 \mathrm{~Hz}, 1 \mathrm{H}), 2.63(\mathrm{~s}, 3 \mathrm{H}) .{ }^{13} \mathrm{C} \mathrm{NMR}$ (150 MHz, $\left.\mathrm{C}_{6} \mathrm{D}_{6}\right) \delta 157.0,140.7,134.4,132.1,130.0,129.0,128.7,128.1,126.9,126.1,121.6,112.2$, 38.2 [carbon attached to boron was not observed due to quadrupole broadening caused by the boron nucleus]. ${ }^{11} \mathrm{~B}$ NMR (193 MHz, $\mathrm{C}_{6} \mathrm{D}_{6} / \mathrm{BF}_{3} \cdot \mathrm{OEt}_{2}$ ) $\delta$ 33.0. LRMS (EI) $m / z: 284.1\left(\mathrm{M}^{+}\right.$). HRMS (EI-EB) $m / z:\left(\mathrm{M}^{+}\right)$Calcd. for $\mathrm{C}_{19} \mathrm{H}_{17} \mathrm{BN}_{2}: 284.1485$, found: 284.1481. IR (neat): 749, 1311, 1450, 1495, 1595, $1606,3029 \mathrm{~cm}^{-1}$.

\section{Procedure of stepwise-treatment of 2a with 3c and 3a (Scheme 1, eq. 2).}<smiles>b1c2ccccc2nn1-c1ccccc1</smiles>

$2 a$

$$
\begin{aligned}
& \text { 1) } 3 \mathrm{c}(1.0 \text { equiv. }) \\
& \mathrm{CH}_{2} \mathrm{Cl}_{2}, 0^{\circ} \mathrm{C}, 5 \mathrm{~min} \\
& \text { 2) } 3 \mathbf{a}(1.0 \text { equiv. }) \\
& \mathrm{CH}_{2} \mathrm{Cl}_{2}, 0^{\circ} \mathrm{C}, 5 \mathrm{~min}
\end{aligned}
$$<smiles>CB1c2ccccc2N(c2ccccc2)N1c1ccccc1</smiles>

6

3-Methyl-1,2-diphenyl-2,3-dihydro-1 $\boldsymbol{H}$-benzo[d][1,2,3]diazaborole (6). To a mixture of $\mathbf{2 a}$ (105.9 $\mathrm{mg}, 0.301 \mathrm{mmol})$ and $\mathrm{CH}_{2} \mathrm{Cl}_{2}(1.0 \mathrm{~mL})$ in an oven-dried vial equipped with a stirrer bar was added $3 \mathbf{c}$ solution $(0.165 \mathrm{~mL}, 2.0 \mathrm{M}$ in THF, $0.33 \mathrm{mmol})$ at $0{ }^{\circ} \mathrm{C}$. After being stirred at $0{ }^{\circ} \mathrm{C}$ for $5 \mathrm{~min}$, 3a solution $\left(0.115 \mathrm{~mL}, 3.0 \mathrm{M}\right.$ in $\left.\mathrm{Et}_{2} \mathrm{O}, 0.345 \mathrm{mmol}\right)$ was added at the same temperature. After being further stirred at $0{ }^{\circ} \mathrm{C}$ for $5 \mathrm{~min}, \mathrm{H}_{2} \mathrm{O}(2 \mathrm{~mL})$ was added, and the organic materials were extracted with $\mathrm{CH}_{2} \mathrm{Cl}_{2}(10$ $\mathrm{mL} \times 3)$. The combined organic layer was washed with $\mathrm{H}_{2} \mathrm{O}(10 \mathrm{~mL})$ and brine $(10 \mathrm{~mL})$, dried over $\mathrm{Na}_{2} \mathrm{SO}_{4}$, and concentrated. Yield of 6 was determined to be $90 \%$ by ${ }^{1} \mathrm{H}$ NMR spectroscopy using $1,1,2-$ trichloroethane as an internal standard. The crude material was purified by passing through a short pad of activated alumina (hexane: $\mathrm{CH}_{2} \mathrm{Cl}_{2}=4: 1$ ) to give the solid material, which was recrystallized from $\mathrm{CH}_{2} \mathrm{Cl}_{2}$ /hexane to afford $6(44.7 \mathrm{mg}, 0.157 \mathrm{mmol}, 52 \%)$ as a yellow solid: $\mathrm{Mp} 101-103{ }^{\circ} \mathrm{C}$ $\left(\mathrm{CH}_{2} \mathrm{Cl}_{2}\right.$ /hexane). ${ }^{1} \mathrm{H}$ NMR (400 MHz, $\left.\mathrm{C}_{6} \mathrm{D}_{6}\right) \delta 7.94(\mathrm{t}, J=7.8 \mathrm{~Hz}, 1 \mathrm{H}), 7.30-7.20(\mathrm{~m}, 3 \mathrm{H}), 7.18-7.08$ (m, 2H), 7.08-7.02 (m, 2H), $6.99(\mathrm{t}, J=8.1 \mathrm{~Hz}, 2 \mathrm{H}), 6.92(\mathrm{t}, J=8.1 \mathrm{~Hz}, 2 \mathrm{H}), 6.79-6.72(\mathrm{~m}, 2 \mathrm{H}), 1.03$ (s, 3H). ${ }^{13} \mathrm{C}$ NMR (150 MHz, $\left.\mathrm{C}_{6} \mathrm{D}_{6}\right) \delta 155.5,144.4,141.9,131.4,130.5,129.1,128.8,126.5,125.7$, $124.8,124.7,121.6,112.3$ [carbon attached to boron was not observed due to quadrupole broadening caused by the boron nucleus]. ${ }^{11} \mathrm{~B}$ NMR $\left(193 \mathrm{MHz}, \mathrm{C}_{6} \mathrm{D}_{6} / \mathrm{BF}_{3} \cdot \mathrm{OEt}_{2}\right) \delta$ 37.4. LRMS (EI) $\mathrm{m} / z: 284.2$ $\left(\mathrm{M}^{+}\right)$. HRMS (EI-TOF) $m / z$ : $\left(\mathrm{M}^{+}\right)$Calcd. for $\mathrm{C}_{19} \mathrm{H}_{17} \mathrm{BN}_{2}$ : 284.1485, found: 284.14840. IR (neat): 745, $762,1309,1448,1456,1486,1594,3039 \mathrm{~cm}^{-1}$. 


\section{X-Ray Crystallographic Data for 2a}

The structure of $\mathbf{2 a}$ was also elucidated by X-ray analysis. The single crystal for the analysis was grown by vapor diffusion of hexane to a $\mathrm{CH}_{2} \mathrm{Cl}_{2}-\mathrm{Et}_{2} \mathrm{O}$ solution of 2a. X Ray Crystallography: CCDC2094404 contains the supplementary crystallographic data for this paper. The data can be obtained free of charge from the Cambridge Crystallographic Data Centre via www.ccdc.cam.ac.uk/data_request/cif.

\section{Crystal data and structure refinement}

Compound: 2a

Formula: C12 H9 B Br2 N2

Formula weight: 351.84

Wave length: $=0.71075$

Crystal system: triclinic

Space group: $\mathrm{P}-1$

Color of crystal: Brown

Unit cell parameters: $\quad \mathrm{a}=7.6667(9) \AA \quad \alpha=101.134(4)^{\circ}$

$$
\begin{array}{ll}
\mathrm{b}=8.6489(10) \AA & \beta=100.534(3)^{\circ} \\
\mathrm{c}=9.7974(11) \AA & \gamma=94.669(4)^{\circ}
\end{array}
$$

Temperature of data collection: $173(\mathrm{~K})$

Values of $\mathrm{Z}, \mathrm{R}, \mathrm{GOF}: \mathrm{Z}=2$

$$
\mathrm{R}(\text { reflections })=0.0714(2119), \mathrm{wR} 2(\text { reflections })=0.2128(2827)
$$

$$
\mathrm{GOF}=1.096
$$

Radiation type: Mo K/a

Radiation source: sealed X-ray tube

Radiation monochromator: graphite

Measurement device type: Rigaku R-AXIS RAPID

Computing structure solution: SHELXS

Computing structure refinement: SHELXL-97 (Sheldrick, 1997) 
ORTEP views of $\mathbf{2 a}$

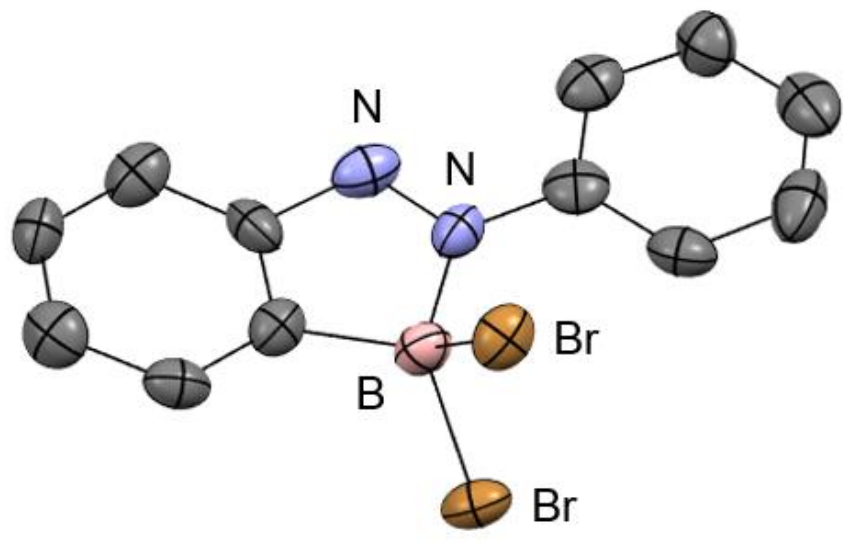

Thermal ellipsoid plot of $\mathbf{2 a}$ at $50 \%$ probability. 


\section{X-Ray Crystallographic Data for 4gc}

The structure of $4 \mathrm{gc}$ was also elucidated by X-ray analysis. The single crystal for the analysis was grown by vapor diffusion of hexane to a $\mathrm{CH}_{2} \mathrm{Cl}_{2}-\mathrm{Et}_{2} \mathrm{O}$ solution of 4gc. X Ray Crystallography: CCDC2094407 contains the supplementary crystallographic data for this paper. The data can be obtained free of charge from the Cambridge Crystallographic Data Centre via www.ccdc.cam.ac.uk/data_request/cif.

\section{Crystal data and structure refinement}

Compound: 4gc

Formula: C24 H17 B Cl2 N2

Formula weight: 415.11

Wave length: $=0.71075$

Crystal system: monoclinic

Space group: P 21/n

Color of crystal: colorless

Unit cell parameters: $\quad \mathrm{a}=9.6103(10) \AA \quad \alpha=90^{\circ}$

$$
\begin{array}{ll}
\mathrm{b}=16.2456(14) \AA & \beta=92.912(4)^{\circ} \\
\mathrm{c}=12.8843(14) \AA & \gamma=90^{\circ}
\end{array}
$$

Temperature of data collection: $173(\mathrm{~K})$

Values of $Z, R, G O F: ~ Z=4$

$$
\mathrm{R}(\text { reflections })=0.0669(4013), \mathrm{wR} 2(\text { reflections })=0.1520(4568)
$$

$$
\mathrm{GOF}=1.266
$$

Radiation type: Mo K/a

Radiation source: sealed X-ray tube

Radiation monochromator: graphite

Measurement device type: Rigaku R-AXIS RAPID

Computing structure solution: SHELXD

Computing structure refinement: SHELXL-97 (Sheldrick, 1997) 


\section{ORTEP views of $\mathbf{4 g c}$}

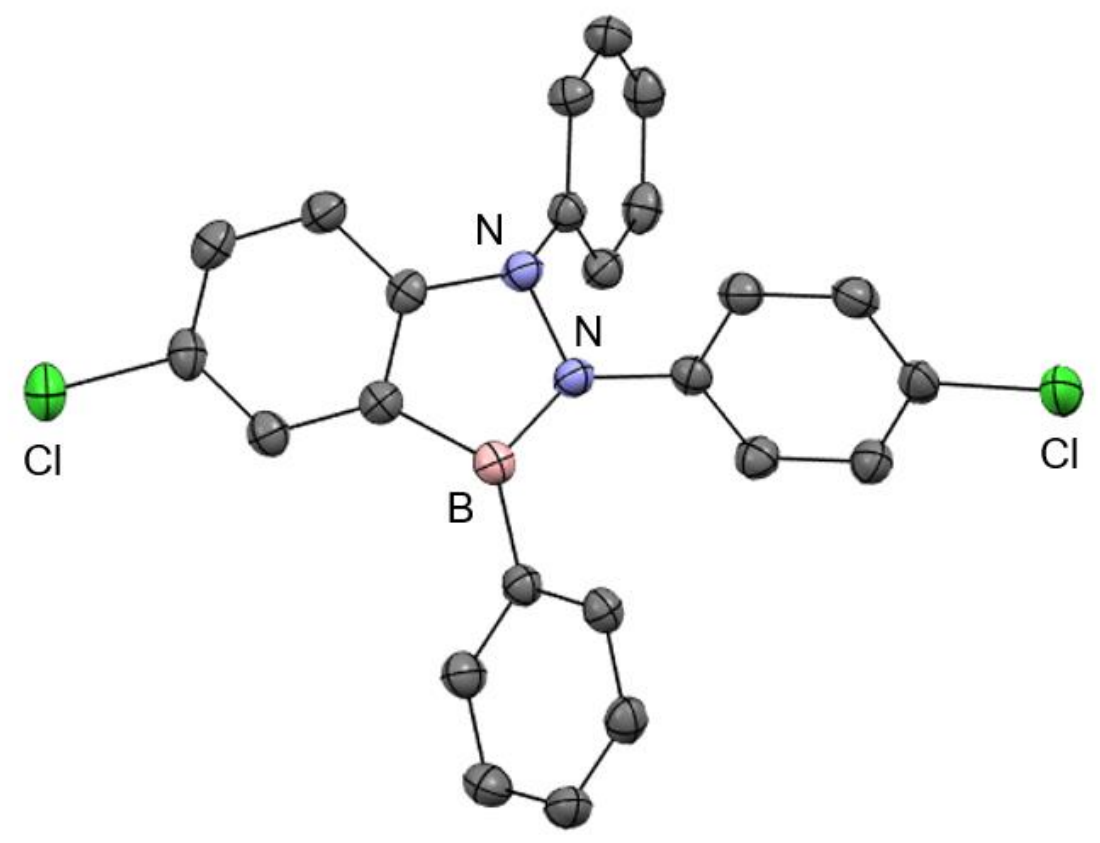

Thermal ellipsoid plot of $\mathbf{4 g c}$ at 50\% probability. 


\section{Theoretical Calculations}

All theoretical calculations were performed using Gaussian 09 and 16 program packages. ${ }^{10,11}$ Geometry optimizations and frequency analyses for 1a, 2a, and 4ac were carried out at B3LYP/6$311 \mathrm{G}(\mathrm{d}, \mathrm{p})$ level of theory, wherein the geometries obtained from X-ray single crystal analysis of 2a and $\mathbf{4 g c}$ were used for the geometry optimizations of $\mathbf{2 a}$ and $\mathbf{4 a c}$, respectively, and their energies were calculated at the same level of theory. Atomic coordinates of the optimized structures are listed in Tables S4-S6. Frontier molecular orbitals of the optimized structures of 1a, 2a, and 4ac and their energy levels were shown in Figures 3, S4, and S5. Nucleus independent chemical shifts (NICS) were calculated using the gauge invariant atomic orbital (GIAO) approach at the GIAO-B3LYP/6$311+\mathrm{G}(2 \mathrm{~d}, \mathrm{p})$ level of theory and performed with the Aroma package (1.0) of Stanger and coworkers using $\mathrm{cphf}=$ grid $=$ fine and integral $=$ grid $=$ ultrafine keywords. ${ }^{12}$ NICS $(1)$ zz values were averaged by two positions (above and below the plane) of all the equivalent rings (Figure S6).

Table S4. Atomic coordinates of $\mathbf{1 a}$<smiles>c1ccc(/N=N/c2ccccc2)cc1</smiles>

$1 \mathrm{a}$

\begin{tabular}{ccccc}
\hline Tag & Atom & $\mathrm{X}$ & $\mathrm{Y}$ & $\mathrm{Z}$ \\
\hline 1 & $\mathrm{C}$ & 4.530577 & 0.20026 & 0.00004754 \\
2 & $\mathrm{C}$ & 4.010688 & -1.09205 & -0.00073392 \\
3 & $\mathrm{C}$ & 2.632549 & -1.28398 & -0.00072745 \\
4 & $\mathrm{C}$ & 1.768272 & -0.18469 & -0.00007197 \\
5 & $\mathrm{C}$ & 2.293454 & 1.116289 & 0.00075462 \\
6 & $\mathrm{C}$ & 3.668159 & 1.301031 & 0.00082296 \\
7 & $\mathrm{H}$ & 5.603851 & 0.353826 & 0.00009237 \\
8 & $\mathrm{H}$ & 4.676542 & -1.94733 & -0.00130227 \\
9 & $\mathrm{H}$ & 2.198665 & -2.27674 & -0.00126628 \\
10 & $\mathrm{H}$ & 1.609303 & 1.954149 & 0.00135017 \\
11 & $\mathrm{H}$ & 4.076174 & 2.3057 & 0.00149422 \\
12 & $\mathrm{~N}$ & 0.38431 & -0.49511 & -0.00008696 \\
13 & $\mathrm{~N}$ & -0.38427 & 0.494946 & -0.00015254 \\
14 & $\mathrm{C}$ & -1.76826 & 0.184583 & -0.0000754 \\
15 & $\mathrm{C}$ & -2.63242 & 1.283954 & -0.00074601 \\
\hline
\end{tabular}




\begin{tabular}{lllll}
\hline 16 & $\mathrm{C}$ & -2.29358 & -1.11635 & 0.000735 \\
17 & $\mathrm{C}$ & -4.01058 & 1.092149 & -0.00071904 \\
18 & $\mathrm{H}$ & -2.19844 & 2.276671 & -0.00130684 \\
19 & $\mathrm{C}$ & -3.6683 & -1.30096 & 0.00081096 \\
20 & $\mathrm{H}$ & -1.60951 & -1.95428 & 0.00128538 \\
21 & $\mathrm{C}$ & -4.5306 & -0.2001 & 0.00006872 \\
22 & $\mathrm{H}$ & -4.67635 & 1.947499 & -0.00129211 \\
23 & $\mathrm{H}$ & -4.07642 & -2.30559 & 0.00147543 \\
24 & $\mathrm{H}$ & -5.60389 & -0.35355 & 0.00015041 \\
\hline
\end{tabular}

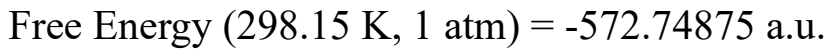

Table S5. Atomic coordinates of $\mathbf{2 a}$<smiles></smiles>

2a

\begin{tabular}{ccccc}
\hline Tag & Atom & $\mathrm{X}$ & $\mathrm{Y}$ & $\mathrm{Z}$ \\
\hline 1 & $\mathrm{Br}$ & 0.211508 & -1.55024 & -1.70682 \\
2 & $\mathrm{Br}$ & 0.210689 & -1.54909 & 1.707571 \\
3 & $\mathrm{~N}$ & 0.223549 & 1.904009 & -0.0006 \\
4 & $\mathrm{C}$ & 2.549058 & 2.658772 & -0.00058 \\
5 & $\mathrm{H}$ & 2.251816 & 3.700365 & -0.00093 \\
6 & $\mathrm{C}$ & 3.238166 & -0.09159 & 0.00032 \\
7 & $\mathrm{H}$ & 3.526661 & -1.13636 & 0.000633 \\
8 & $\mathrm{~N}$ & -0.44075 & 0.816713 & -0.0004 \\
9 & $\mathrm{C}$ & -1.86185 & 0.896563 & -0.00038 \\
10 & $\mathrm{C}$ & -3.8836 & 2.207448 & $5.32 \mathrm{E}-05$ \\
11 & $\mathrm{H}$ & -4.37456 & 3.173456 & 0.000431 \\
12 & $\mathrm{C}$ & 1.585857 & 1.644001 & -0.00038 \\
13 & $\mathrm{C}$ & -2.49907 & 2.146218 & $5.12 \mathrm{E}-05$ \\
14 & $\mathrm{H}$ & -1.90115 & 3.04619 & 0.000388 \\
15 & $\mathrm{C}$ & 1.899355 & 0.27278 & 0.0000827 \\
\hline
\end{tabular}




\begin{tabular}{lllll}
\hline 16 & $\mathrm{C}$ & 4.217054 & 0.906666 & 0.000145 \\
17 & $\mathrm{~B}$ & 0.519102 & -0.49785 & 0.000102 \\
18 & $\mathrm{C}$ & -2.61695 & -0.28121 & -0.00077 \\
19 & $\mathrm{H}$ & -2.1295 & -1.24373 & -0.00104 \\
20 & $\mathrm{C}$ & 3.880409 & 2.268582 & -0.00029 \\
21 & $\mathrm{H}$ & 4.665183 & 3.015375 & -0.00038 \\
22 & $\mathrm{C}$ & -4.64307 & 1.035051 & -0.00039 \\
23 & $\mathrm{C}$ & -4.00564 & -0.20204 & -0.00079 \\
24 & $\mathrm{H}$ & -4.58739 & -1.11586 & -0.00116 \\
25 & $\mathrm{H}$ & -5.72549 & 1.089487 & -0.00035 \\
26 & $\mathrm{H}$ & 5.264125 & 0.624222 & 0.000336 \\
\hline
\end{tabular}

Free Energy $(298.15 \mathrm{~K}, 1 \mathrm{~atm})=-5745.41696$ a.u.

Table S6. Atomic coordinates of $\mathbf{4 a c}$<smiles>PC1=CC=CN(c2ccccc2)c2ccccc21</smiles>

$4 a c$

\begin{tabular}{ccccc}
\hline Tag & Atom & $\mathrm{X}$ & $\mathrm{Y}$ & $\mathrm{Z}$ \\
\hline 1 & $\mathrm{~N}$ & 0.026049 & -0.16976 & -0.14379 \\
2 & $\mathrm{C}$ & -2.39371 & 0.109912 & 0.123969 \\
3 & $\mathrm{C}$ & 1.04999 & 3.418664 & -0.23207 \\
4 & $\mathrm{H}$ & 2.111633 & 3.588428 & -0.08633 \\
5 & $\mathrm{C}$ & -1.71313 & 2.989302 & -0.62385 \\
6 & $\mathrm{H}$ & -2.77433 & 2.831316 & -0.76867 \\
7 & $\mathrm{~N}$ & -1.16421 & 0.553051 & -0.47692 \\
8 & $\mathrm{C}$ & -4.7687 & -0.26422 & -0.08983 \\
9 & $\mathrm{H}$ & -5.66798 & -0.24865 & -0.69504 \\
10 & $\mathrm{C}$ & -2.44662 & -0.31201 & 1.454097 \\
11 & $\mathrm{H}$ & -1.5415 & -0.32207 & 2.048311 \\
12 & $\mathrm{C}$ & -0.02571 & -1.58234 & -0.30893 \\
13 & $\mathrm{C}$ & 0.193478 & 4.492447 & -0.42513 \\
\hline
\end{tabular}




\begin{tabular}{|c|c|c|c|c|}
\hline 14 & $\mathrm{C}$ & 0.579153 & -2.40516 & 0.644472 \\
\hline 15 & $\mathrm{H}$ & 1.057585 & -1.95644 & 1.505824 \\
\hline 16 & $\mathrm{C}$ & 3.25885 & -0.72625 & -0.61598 \\
\hline 17 & $\mathrm{H}$ & 2.671014 & -1.28398 & -1.33661 \\
\hline 18 & $\mathrm{C}$ & 2.650102 & 0.290299 & 0.141049 \\
\hline 19 & $\mathrm{C}$ & -3.55342 & 0.1226 & -0.65079 \\
\hline 20 & $\mathrm{H}$ & -3.49237 & 0.427088 & -1.68884 \\
\hline 21 & $\mathrm{C}$ & -3.65978 & -0.7172 & 2.001668 \\
\hline 22 & $\mathrm{H}$ & -3.69696 & -1.04871 & 3.033243 \\
\hline 23 & $\mathrm{C}$ & -0.05795 & -4.36017 & -0.62567 \\
\hline 24 & $\mathrm{C}$ & 5.387868 & -0.33401 & 0.454633 \\
\hline 25 & $\mathrm{H}$ & 6.438564 & -0.57493 & 0.574291 \\
\hline 26 & $\mathrm{C}$ & -0.83185 & 1.916604 & -0.44902 \\
\hline 27 & $\mathrm{~B}$ & 1.154003 & 0.707936 & -0.03472 \\
\hline 28 & $\mathrm{C}$ & 4.609165 & -1.031 & -0.46784 \\
\hline 29 & $\mathrm{H}$ & 5.054592 & -1.81475 & -1.07118 \\
\hline 30 & $\mathrm{C}$ & 0.545092 & 2.109673 & -0.21745 \\
\hline 31 & $\mathrm{C}$ & -1.18088 & 4.270958 & -0.60895 \\
\hline 32 & $\mathrm{H}$ & -1.84368 & 5.118142 & -0.74829 \\
\hline 33 & $\mathrm{C}$ & 0.572006 & -3.7868 & 0.476748 \\
\hline 34 & $\mathrm{H}$ & 1.050712 & -4.41607 & 1.21847 \\
\hline 35 & $\mathrm{C}$ & 4.808708 & 0.676437 & 1.21882 \\
\hline 36 & $\mathrm{H}$ & 5.407321 & 1.224971 & 1.938214 \\
\hline 37 & $\mathrm{C}$ & -4.82415 & -0.68925 & 1.235194 \\
\hline 38 & $\mathrm{H}$ & -5.76737 & -1.00294 & 1.667371 \\
\hline 39 & $\mathrm{C}$ & 3.460901 & 0.988276 & 1.054475 \\
\hline 40 & $\mathrm{H}$ & 3.026471 & 1.782554 & 1.653155 \\
\hline 41 & $\mathrm{C}$ & -0.67594 & -3.5388 & -1.56701 \\
\hline 42 & $\mathrm{H}$ & -1.16872 & -3.97508 & -2.42867 \\
\hline 43 & $\mathrm{C}$ & -0.65843 & -2.15574 & -1.41662 \\
\hline 44 & $\mathrm{H}$ & -1.12547 & -1.51368 & -2.15244 \\
\hline 45 & $\mathrm{H}$ & 0.578506 & 5.505711 & -0.43181 \\
\hline 46 & $\mathrm{H}$ & -0.07115 & -5.43696 & -0.74833 \\
\hline
\end{tabular}

Free Energy $(298.15 \mathrm{~K}, 1 \mathrm{~atm})=-1060.32038$ a.u. 
Figure S4. Frontier molecular orbitals of 1a and their energies (eV) calculated at the B3LYP/6$311 \mathrm{G}(\mathrm{d}, \mathrm{p})$ level.

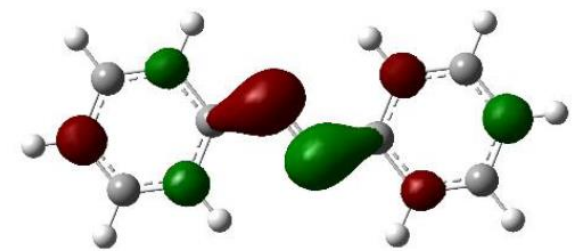

LUMO $(-2.43 \mathrm{eV})$

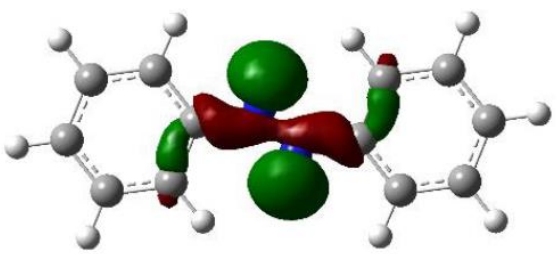

HOMO (-6.35 eV)

Figure S5. Frontier molecular orbitals of 4ac and their energies (eV) calculated at the B3LYP/6311G(d,p) level.

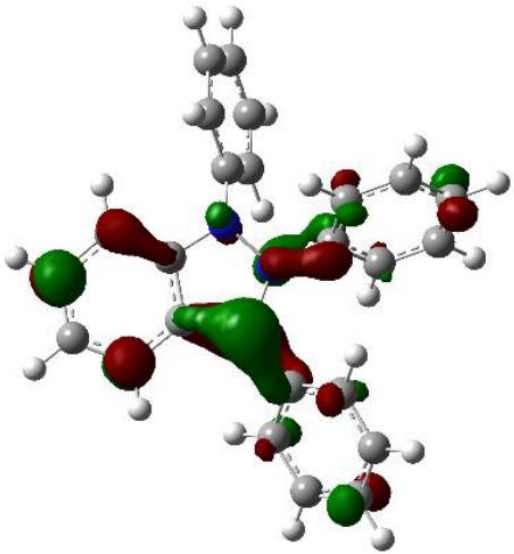

LUMO $(-1.14 \mathrm{eV})$

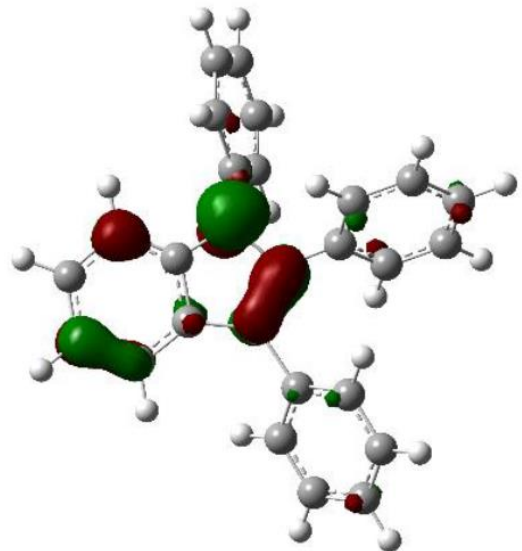

HOMO (-5.62 eV) 


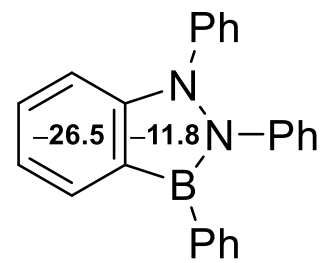

Figure S6. NICS(1)zz value (in ppm) of 4ac. 


\section{References}

1. C. Zhang, N. Jiao, Angew. Chem. Int. Ed. 2010, 49, 6174.

2. Y. Takeda, S. Okumura, S. Minakata, Angew. Chem. Int. Ed. 2012, 51, 7804.

3. H. Song, D. Chen, C. Pi, X. Cui, Y. Wu, J. Org. Chem. 2014, 79, 2955.

4. A. A. John, Q. Lin, J. Org. Chem. 2017, 82, 9873.

5. G. Yu, C. Han, Z. Zhang, J. Chen, X. Yan, B. Zheng, S. Liu, F. Huang, J. Am. Chem. Soc. 2012, 134, 8711.

6. H.-S. Lin, L. A. Paquette, Synth. Commun. 1994, 24, 2503.

7. C. Chen, Y. Pan, H. Zhao, Xin. Xu, Z. Luo, L. Cao, S. Xi, H. Li, L. Xu, Org. Lett. 2018, $20,6799$.

8. J. Yoshino, N. Kano, T. Kawashima, Tetrahedron, 2008, 64, 7774.

9. J. Yoshino, N. Kano, T. Kawashima, Chem. Commun., 2007, 559.

10. Gaussian 09, Revision D.01, M. J. Frisch, G. W. Trucks, H. B. Schlegel, G. E. Scuseria, M. A. Robb, J. R. Cheeseman, G. Scalmani, V. Barone, B. Mennucci, G. A. Petersson, H. Nakatsuji, M. Caricato, X. Li, H. P. Hratchian, A. F. Izmaylov, J. Bloino, G. Zheng, J. L. Sonnenberg, M. Hada, M. Ehara, K. Toyota, R. Fukuda, J. Hasegawa, M. Ishida, T. Nakajima, Y. Honda, O. Kitao, H. Nakai, T. Vreven, J. A. Montgomery, Jr., J. E. Peralta, F. Ogliaro, M. Bearpark, J. J. Heyd, E. Brothers, K. N. Kudin, V. N. Staroverov, R. Kobayashi, J. Normand, K. Raghavachari, A. Rendell, J. C. Burant, S. S. Iyengar, J. Tomasi, M. Cossi, N. Rega, J. M. Millam, M. Klene, J. E. Knox, J. B. Cross, V. Bakken, C. Adamo, J. Jaramillo, R. Gomperts, R. E. Stratmann, O. Yazyev, A. J. Austin, R. Cammi, C. Pomelli, J. W. Ochterski, R. L. Martin, K. Morokuma, V. G. Zakrzewski, G. A. Voth, P. Salvador, J. J. Dannenberg, S. Dapprich, A. D. Daniels, Ö. Farkas, J. B. Foresman, J. V. Ortiz, J. Cioslowski, and D. J. Fox, Gaussian, Inc., Wallingford CT, 2009.

11. Gaussian 16, Revision C.01, M. J. Frisch, G. W. Trucks, H. B. Schlegel, G. E. Scuseria, M. A. Robb, J. R. Cheeseman, G. Scalmani, V. Barone, G. A. Petersson, H. Nakatsuji, X. Li, M. Caricato, A. V. Marenich, J. Bloino, B. G. Janesko, R. Gomperts, B. Mennucci, H. P. Hratchian, J. V. Ortiz, A. F. Izmaylov, J. L. Sonnenberg, D. Williams-Young, F. Ding, F. Lipparini, F. Egidi, J. Goings, B. Peng, A. Petrone, T. Henderson, D. Ranasinghe, V. G. Zakrzewski, J. Gao, N. Rega, G. Zheng, W. Liang, M. Hada,; M. Ehara, K. Toyota, R. Fukuda, J. Hasegawa, M. Ishida, T. Nakajima, Y. Honda, O. Kitao, H. Nakai, T. Vreven, K. Throssell, J. A., Jr. Montgomery, J. E. Peralta, F. Ogliaro, M. J. Bearpark, J. J. Heyd, E. N. Brothers, K. N. Kudin, V. N. Staroverov, T. A. Keith, R. Kobayashi, J. Normand, K. Raghavachari, A. P. Rendell, J. C. Burant, S. S. Iyengar, J. Tomasi, M. Cossi, J. M. Millam, M. Klene, C. Adamo, R. Cammi, J. W. Ochterski, R. L. Martin, K. Morokuma, O. Farkas, J. B. Foresman, D. J. Fox, Gaussian, Inc., Wallingford CT, 2016.

12. (a) A. Rahalkar, A. Stanger, Aroma. http://chemistry.technion.ac.il/ members/amnon-stanger/. (b) A. Stanger, J. Org. Chem. 2006, 71, 883. (c) A. Stanger, J. Org. Chem. 2010, 75, 2281. (d) R. GershoniPoranne, A. Stanger, Chem. Eur. J. 2014, 20, 5673. 
${ }^{1} \mathrm{H}$ NMR spectra of $\mathbf{1 d}\left(\mathrm{CDCl}_{3}, 400 \mathrm{MHz}\right)$

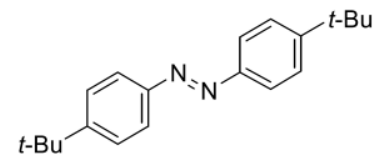

1d

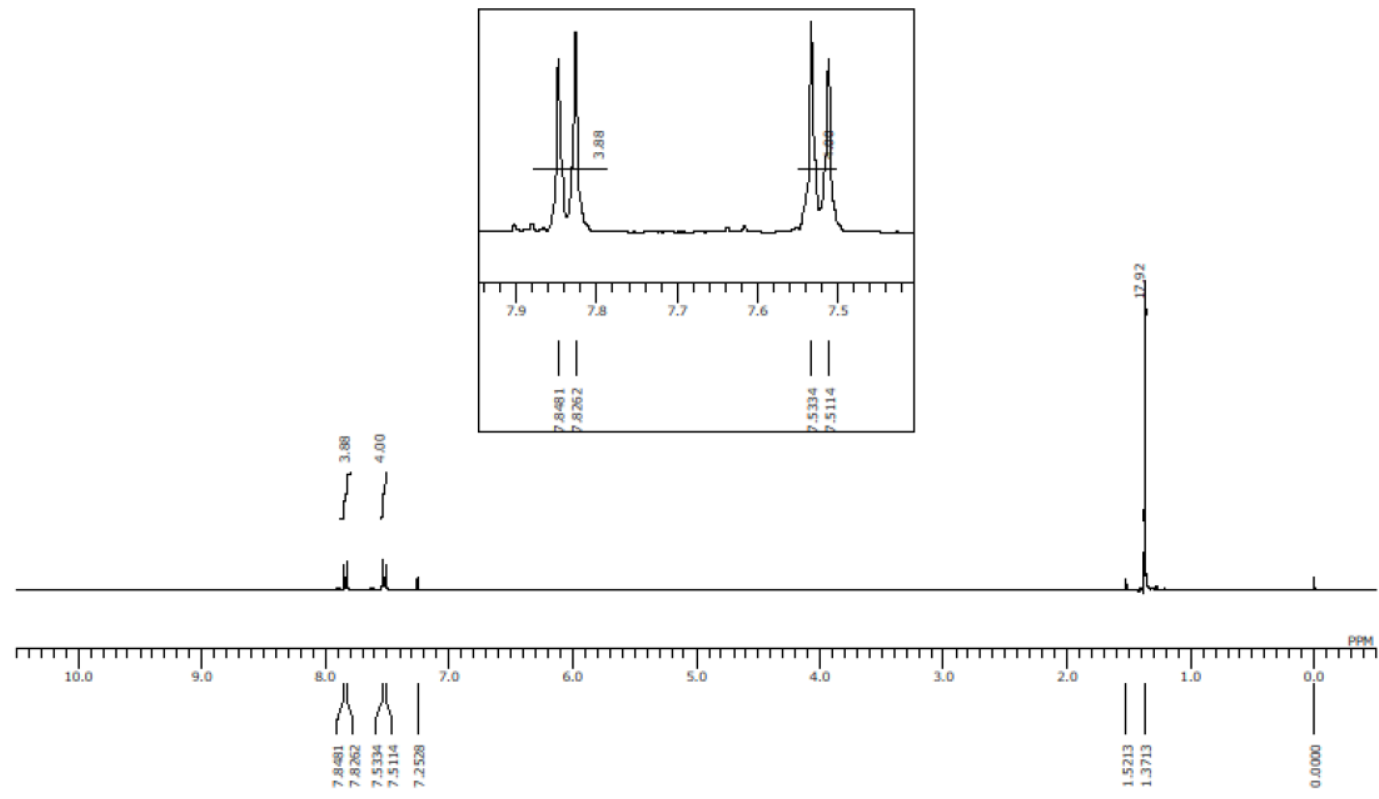

${ }^{13} \mathrm{C}$ NMR spectra of $\mathbf{1 d}\left(\mathrm{CDCl}_{3}, 100 \mathrm{MHz}\right)$

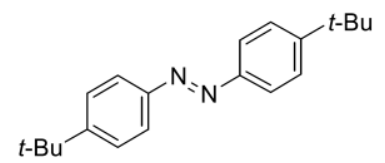

1d

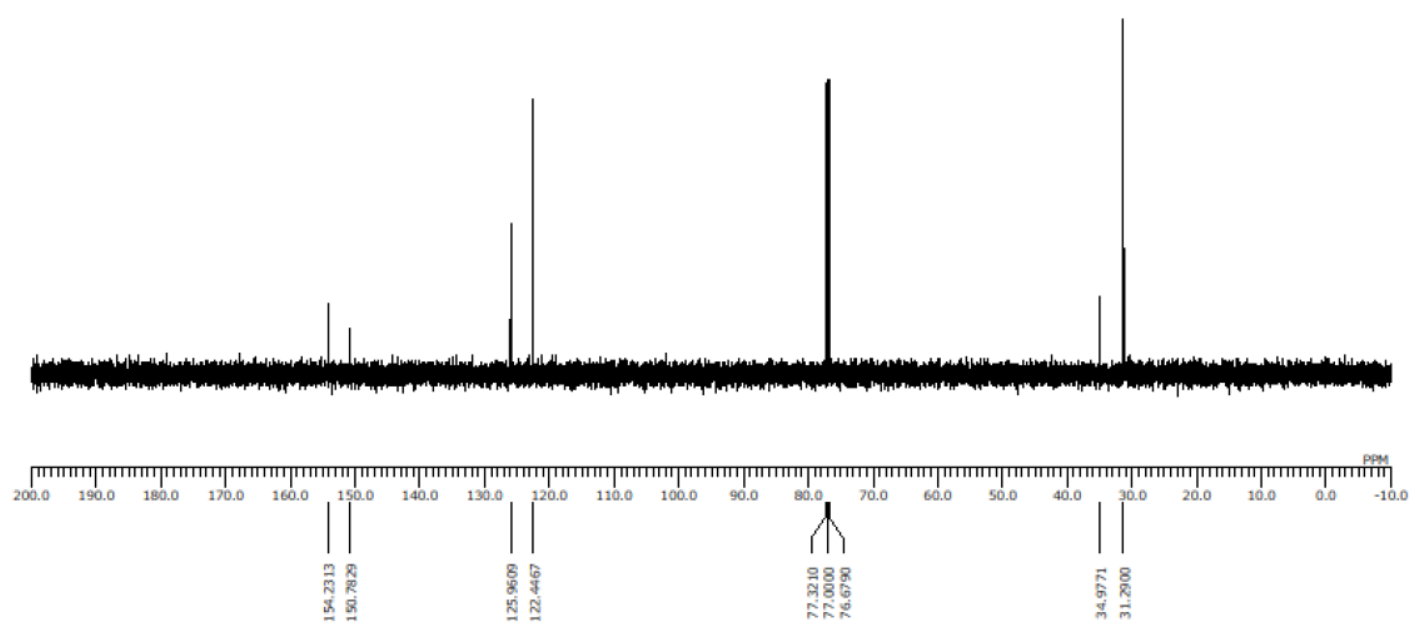


${ }^{1} \mathrm{H}$ NMR spectra of $\mathbf{1 l}\left(\mathrm{CDCl}_{3}, 400 \mathrm{MHz}\right)$
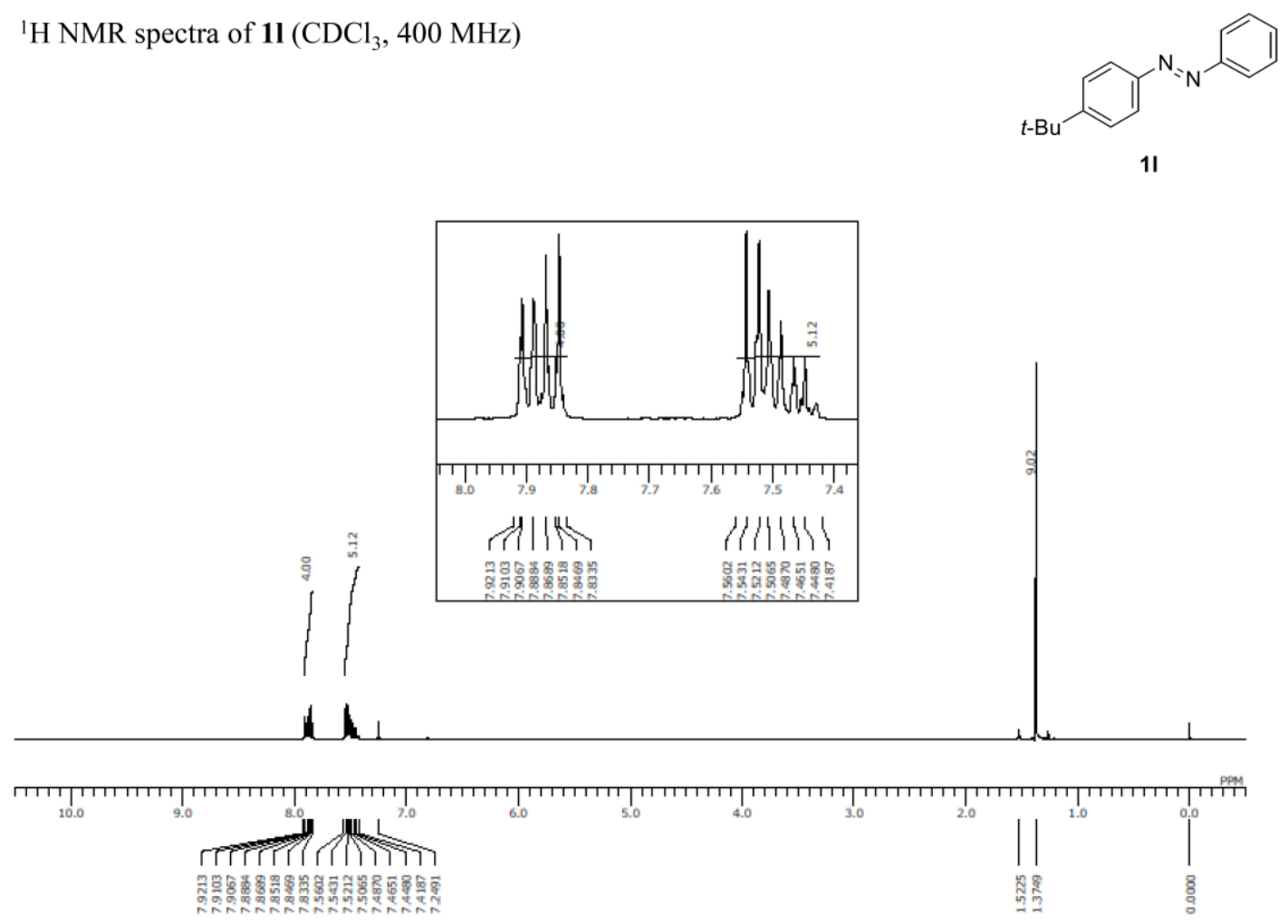

${ }^{13} \mathrm{C}$ NMR spectra of $\mathbf{1 l}\left(\mathrm{CDCl}_{3}, 100 \mathrm{MHz}\right)$

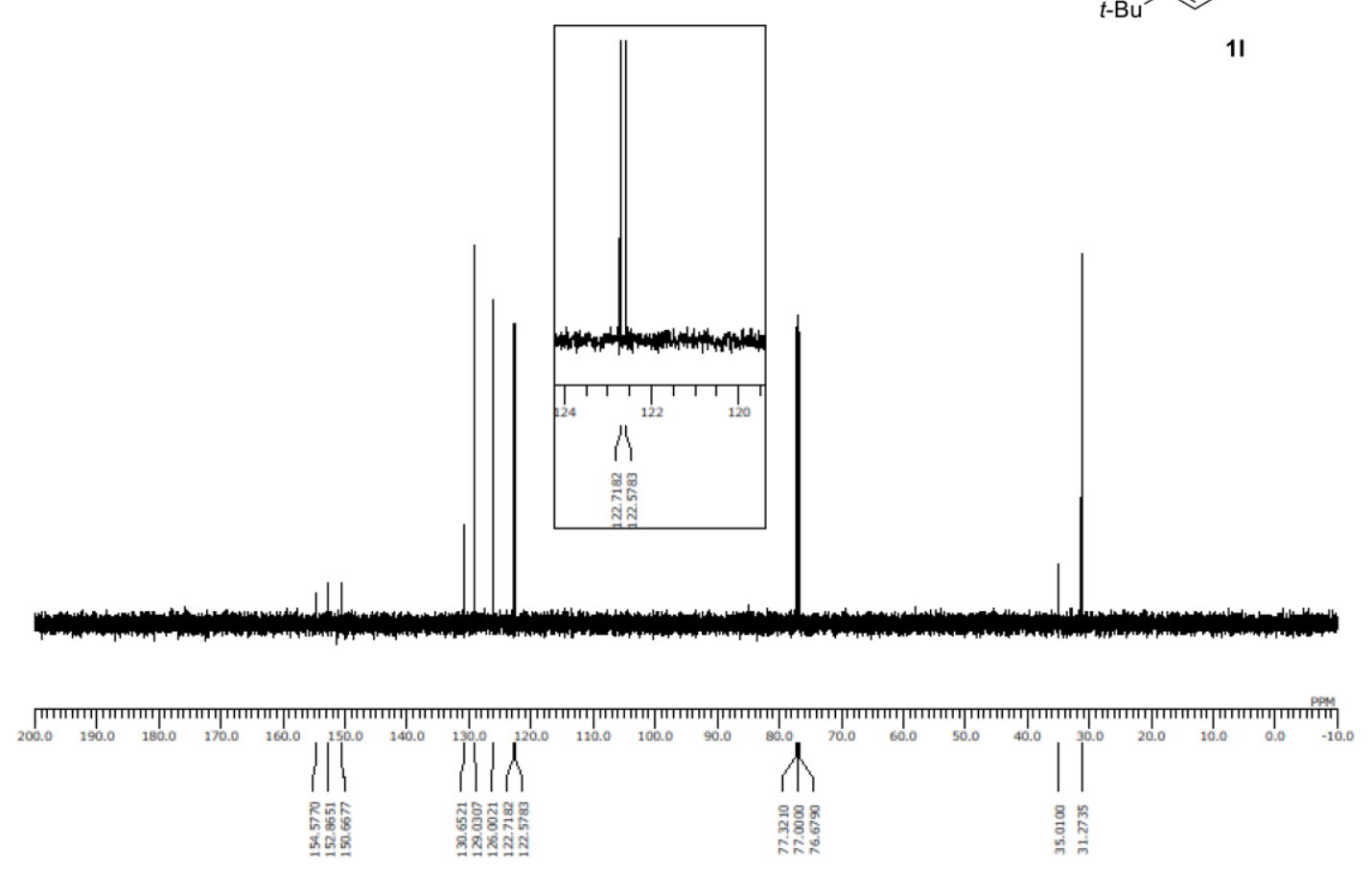


${ }^{1} \mathrm{H}$ NMR spectra of $\mathbf{2 a}\left(\mathrm{CD}_{2} \mathrm{Cl}_{2}, 600 \mathrm{MHz}\right)$

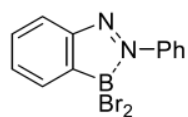

2a
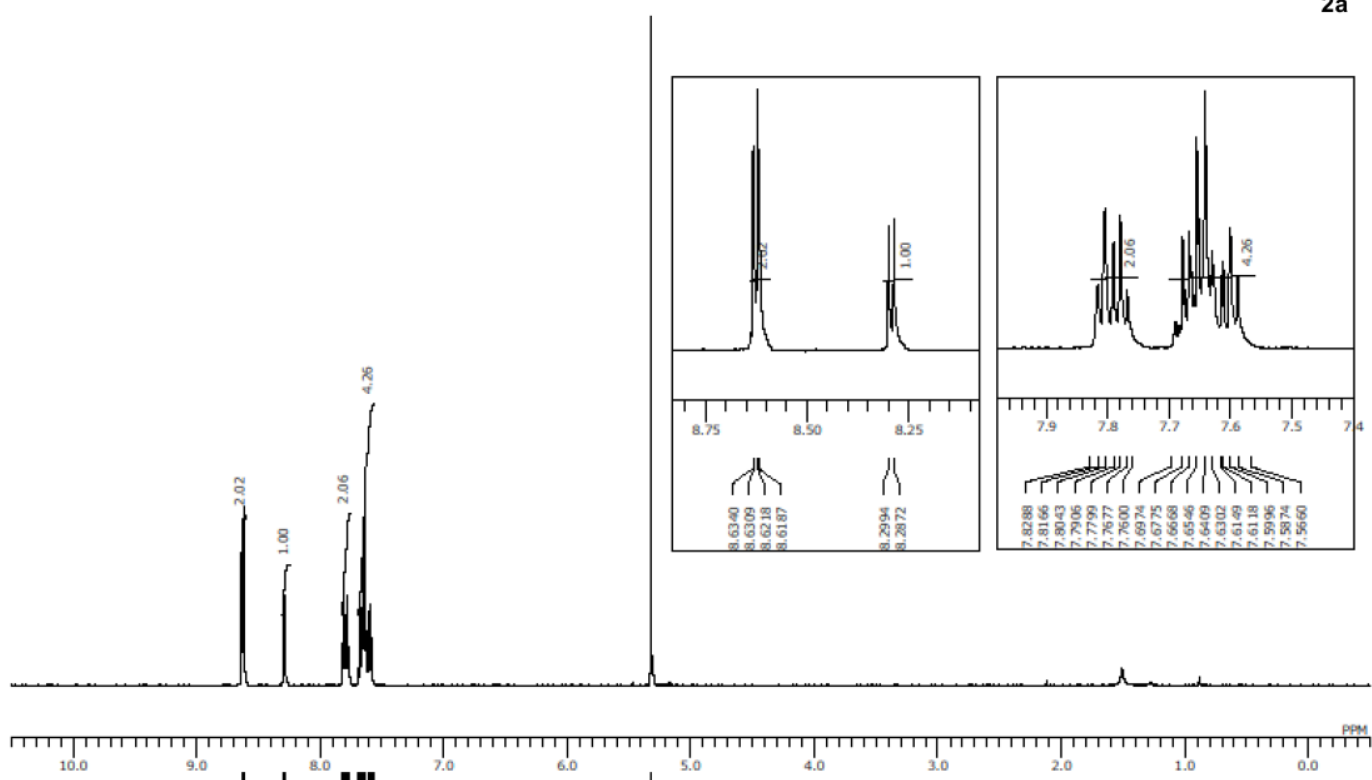

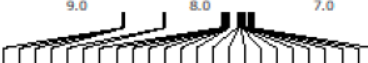

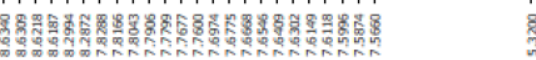

${ }^{13} \mathrm{C}$ NMR spectra of $\mathbf{2 a}\left(\mathrm{CD}_{2} \mathrm{Cl}_{2}, 150 \mathrm{MHz}\right)$

"N-Ph

2a

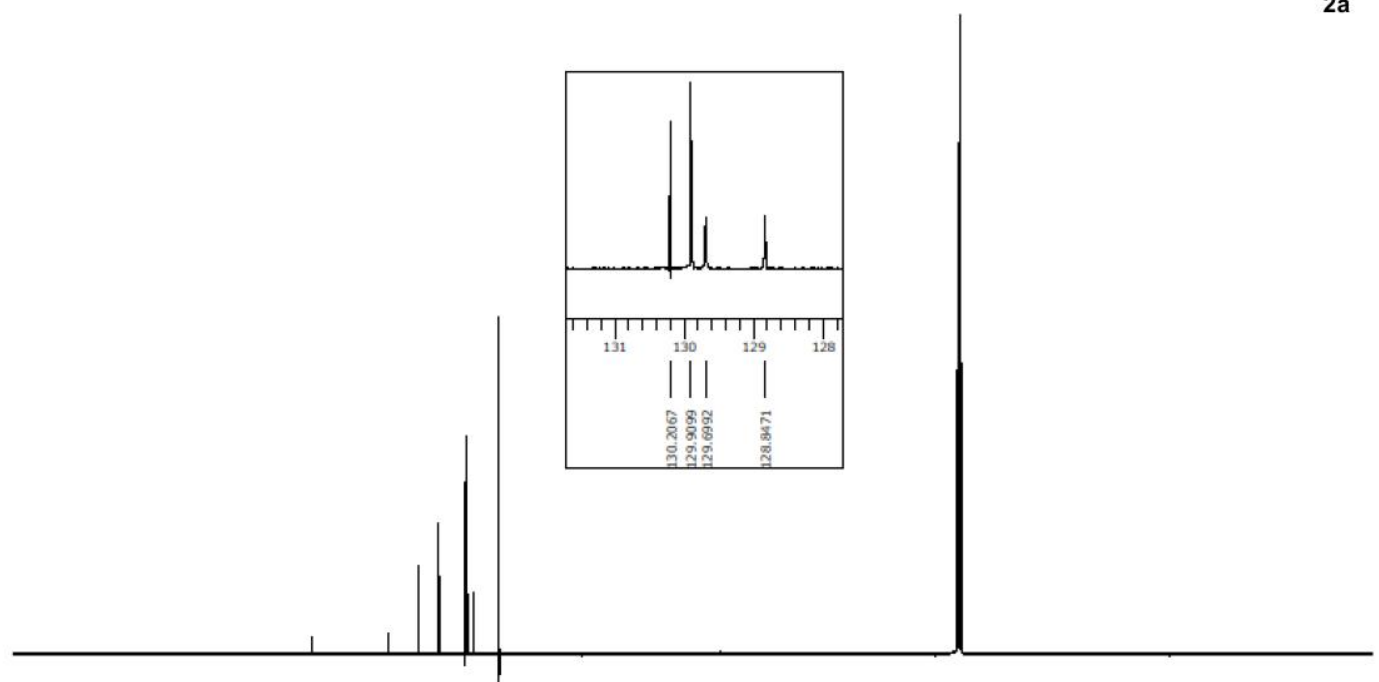

[

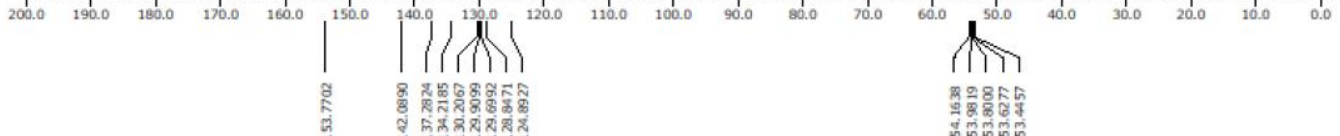


${ }^{11} \mathrm{~B}$ NMR spectra of $\mathbf{2 a}\left(\mathrm{CD}_{2} \mathrm{Cl}_{2}, 193 \mathrm{MHz}\right)$

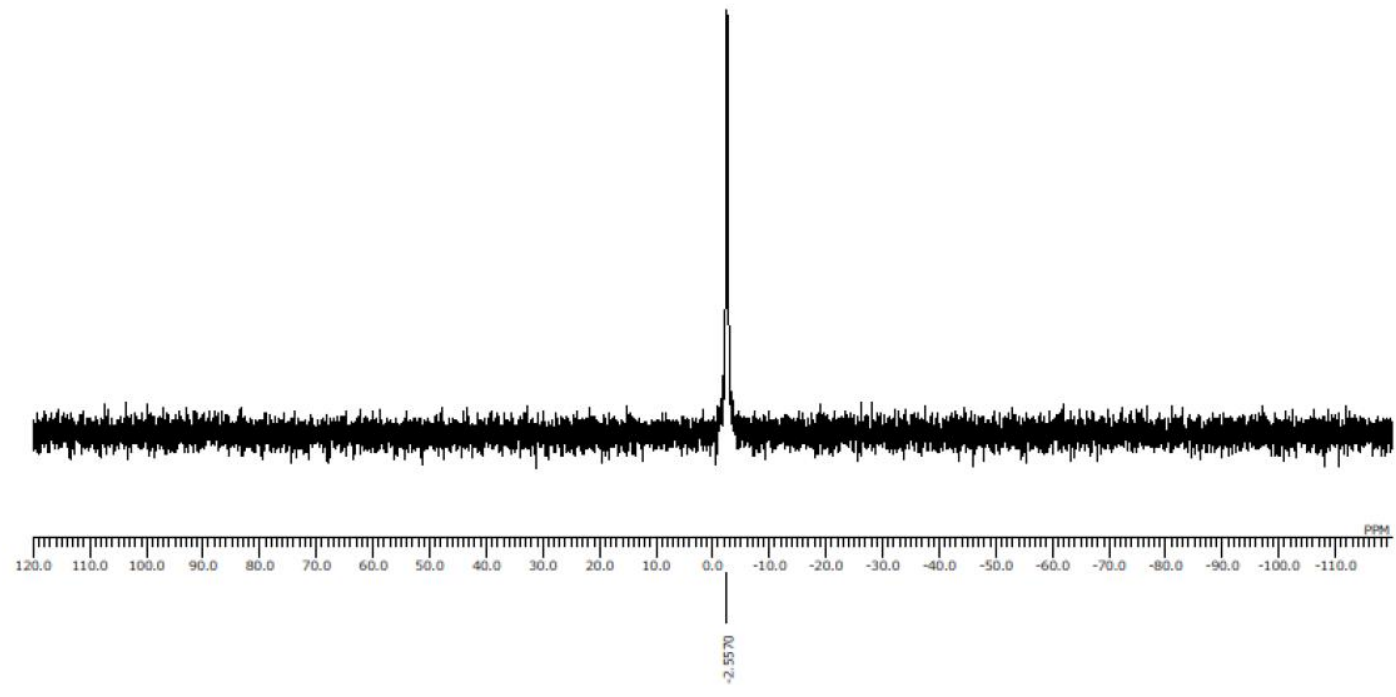

${ }^{1} \mathrm{H}$ NMR spectra of $\mathbf{2} \mathbf{b}\left(\mathrm{CD}_{2} \mathrm{Cl}_{2}, 600 \mathrm{MHz}\right)$
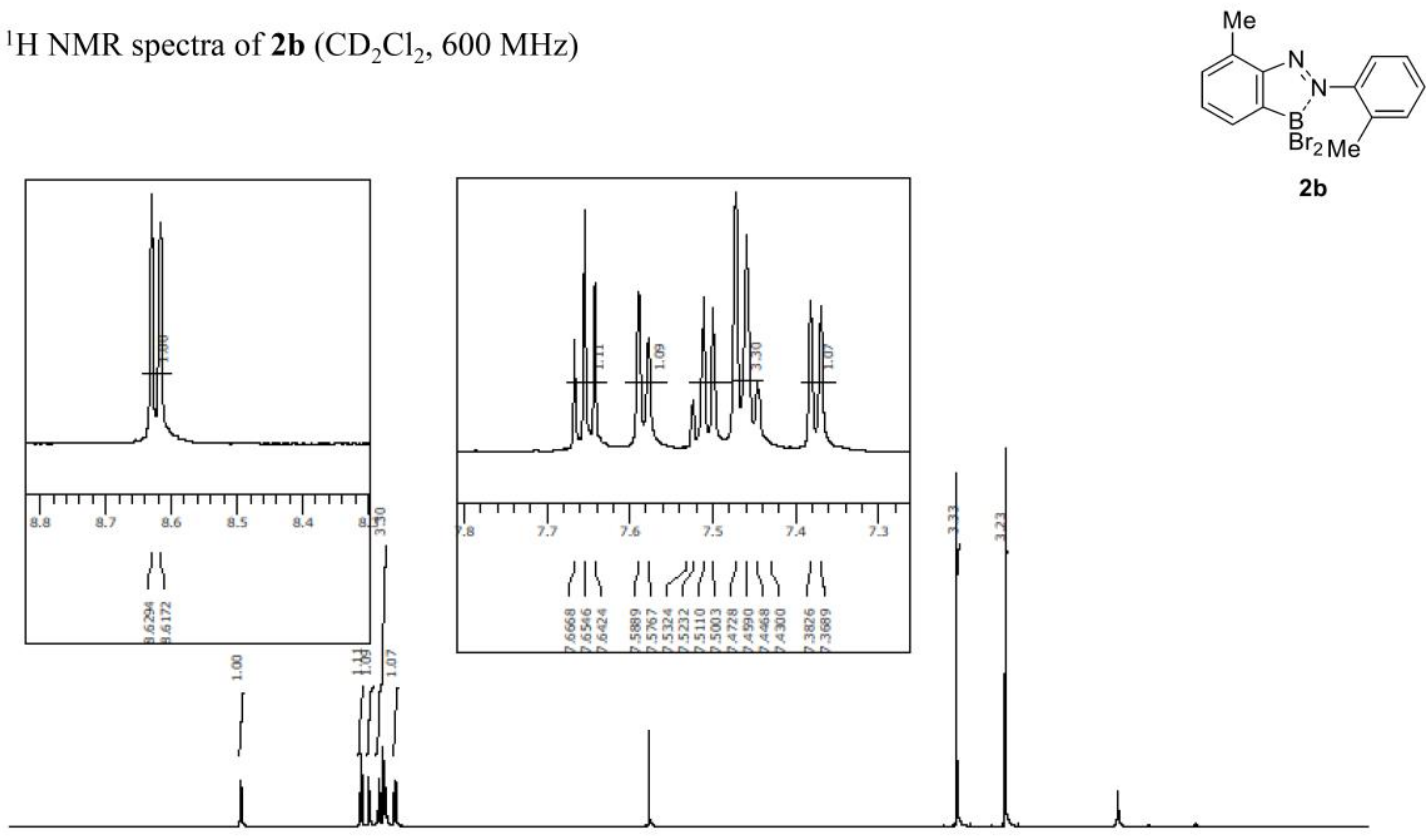

2b

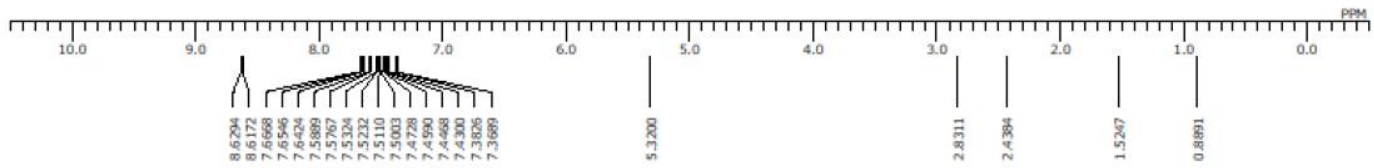


${ }^{13} \mathrm{C}$ NMR spectra of $\mathbf{2 b}\left(\mathrm{CD}_{2} \mathrm{Cl}_{2}, 150 \mathrm{MHz}\right)$

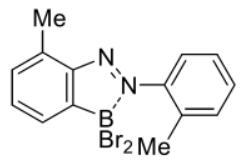

$2 b$

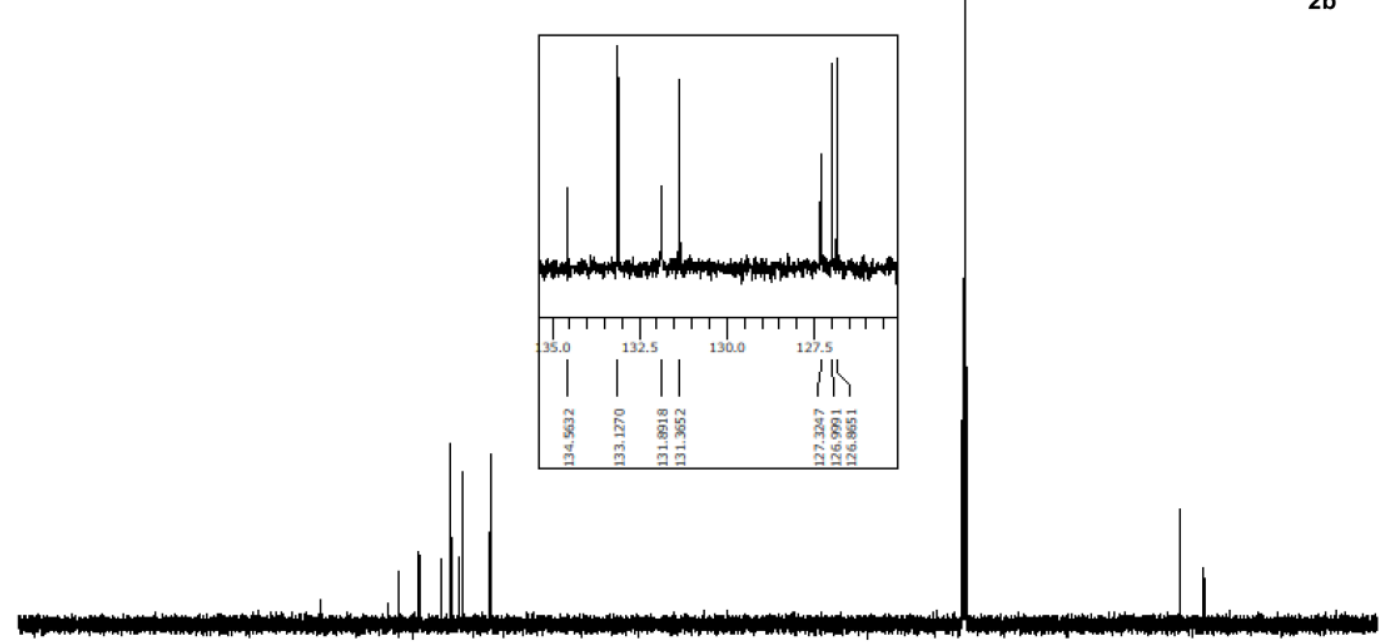

[

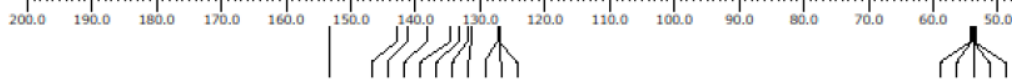

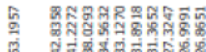

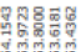

${ }^{11} \mathrm{~B}$ NMR spectra of $\mathbf{2 b}\left(\mathrm{CD}_{2} \mathrm{Cl}_{2}, 193 \mathrm{MHz}\right)$

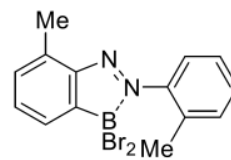

2b

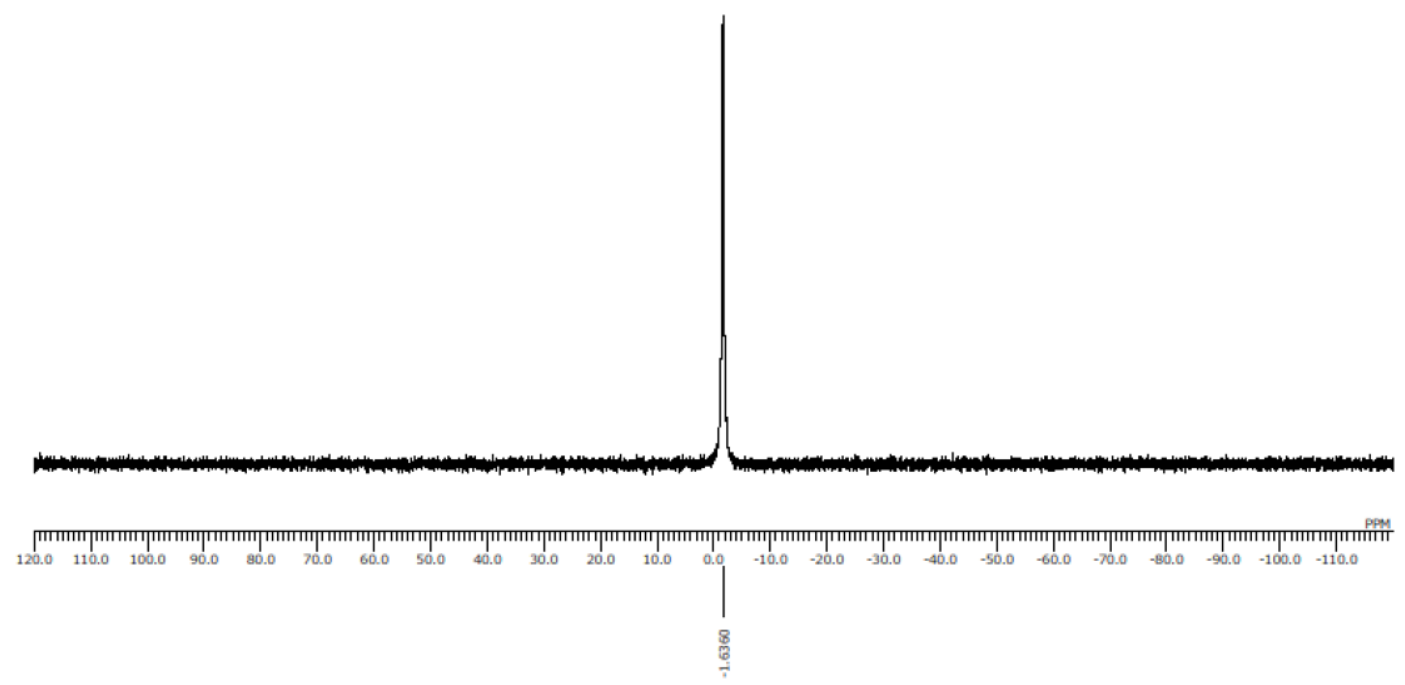


${ }^{1} \mathrm{H}$ NMR spectra of $\mathbf{2 c}\left(\mathrm{CD}_{2} \mathrm{Cl}_{2}, 600 \mathrm{MHz}\right)$

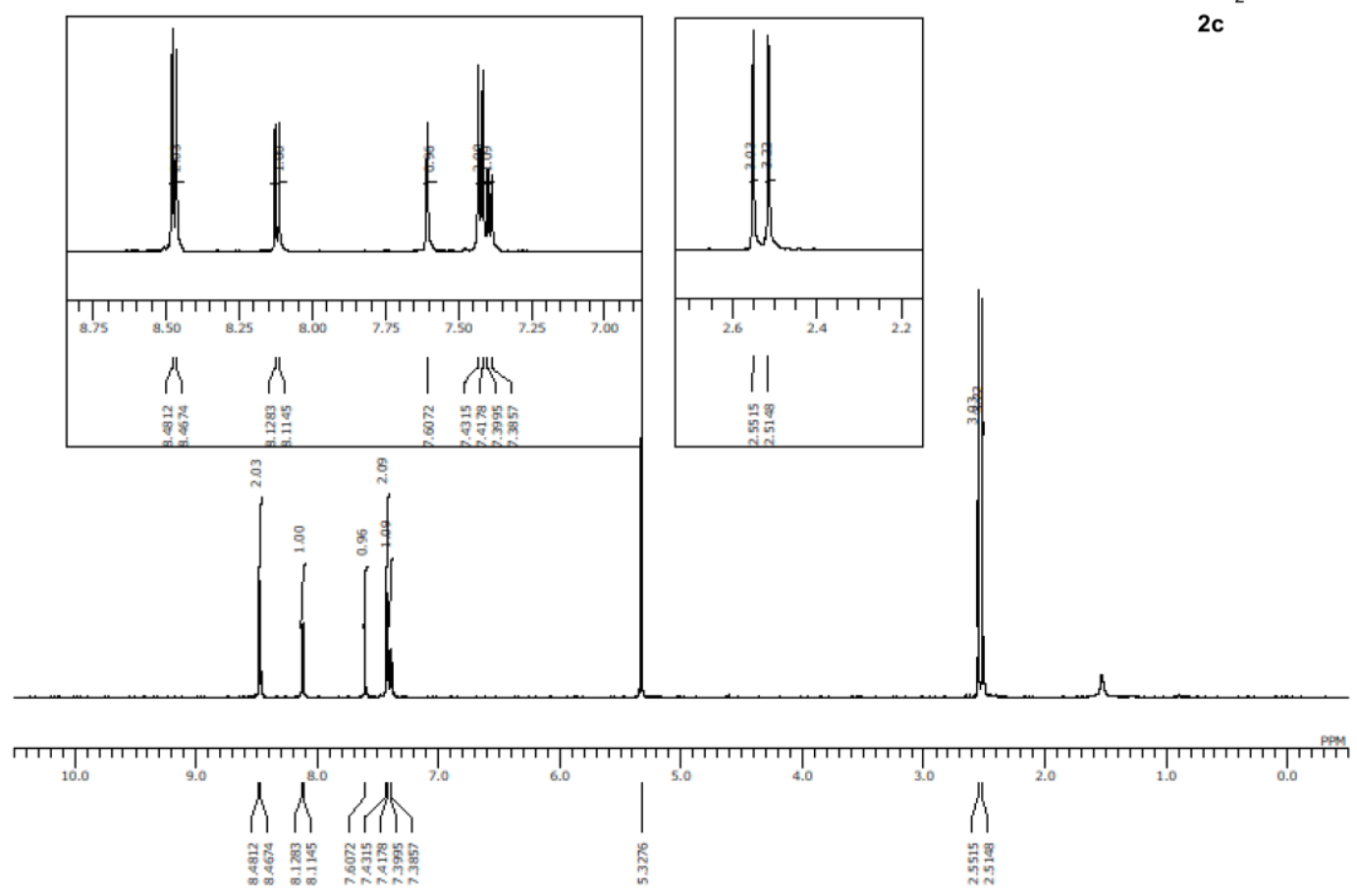

${ }^{13} \mathrm{C}$ NMR spectra of $\mathbf{2 c}\left(\mathrm{CD}_{2} \mathrm{Cl}_{2}, 150 \mathrm{MHz}\right)$
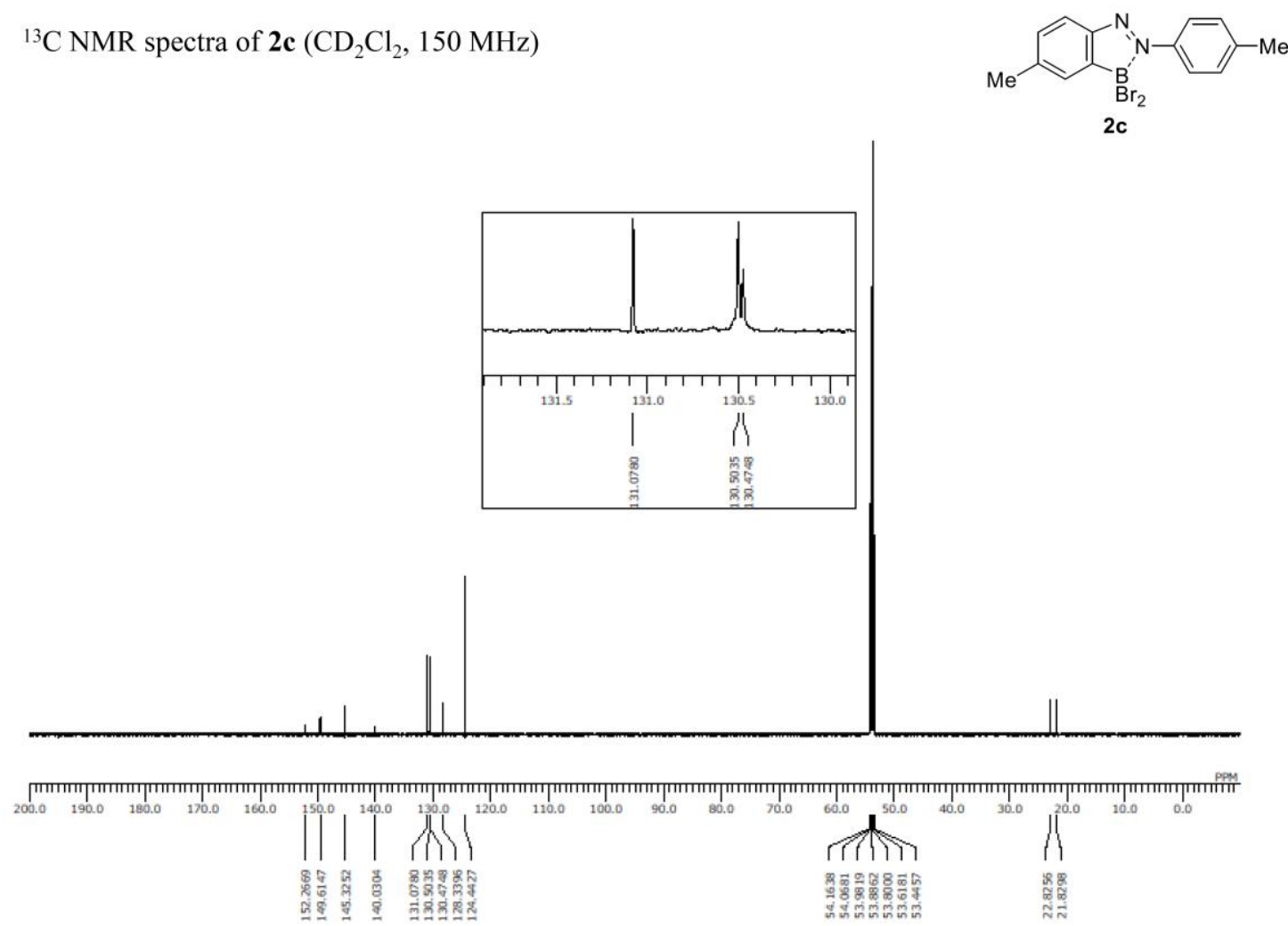

2c 
${ }^{11} \mathrm{~B}$ NMR spectra of $\mathbf{2 c}\left(\mathrm{CD}_{2} \mathrm{Cl}_{2}, 193 \mathrm{MHz}\right)$
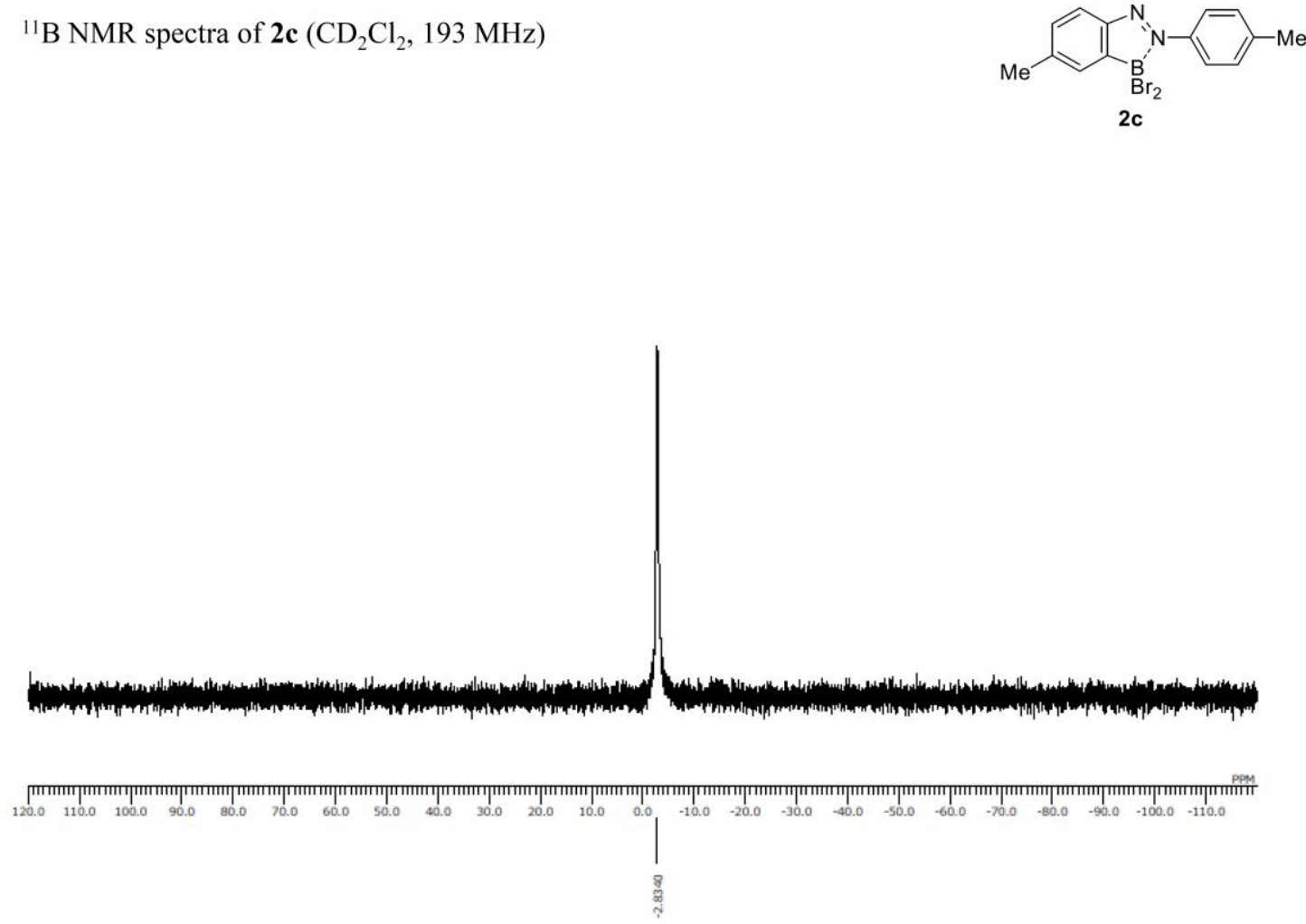

${ }^{1} \mathrm{H}$ NMR spectra of $\mathbf{2 d}\left(\mathrm{CD}_{2} \mathrm{Cl}_{2}, 600 \mathrm{MHz}\right)$
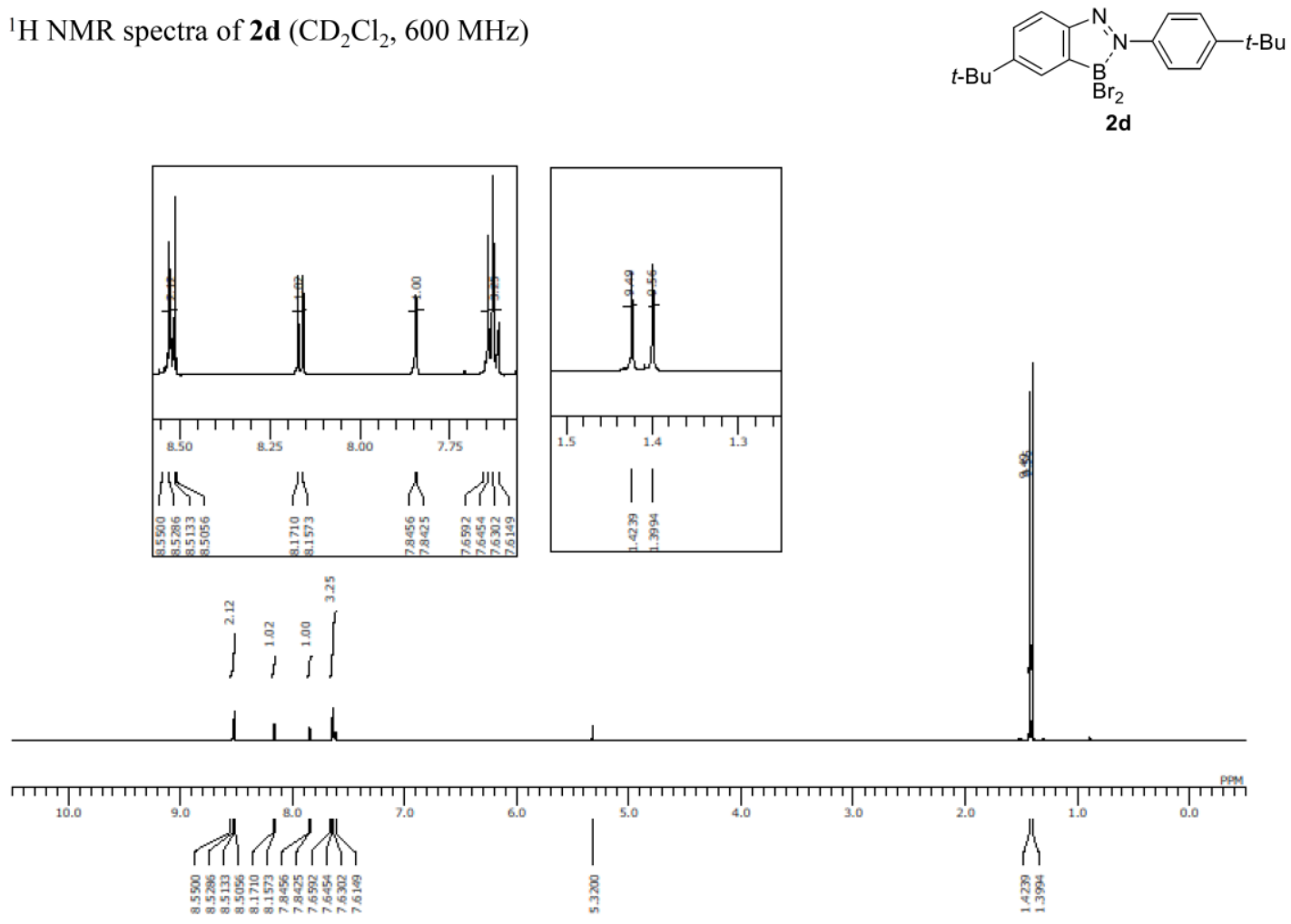
${ }^{13} \mathrm{C}$ NMR spectra of $\mathbf{2 d}\left(\mathrm{CD}_{2} \mathrm{Cl}_{2}, 150 \mathrm{MHz}\right)$
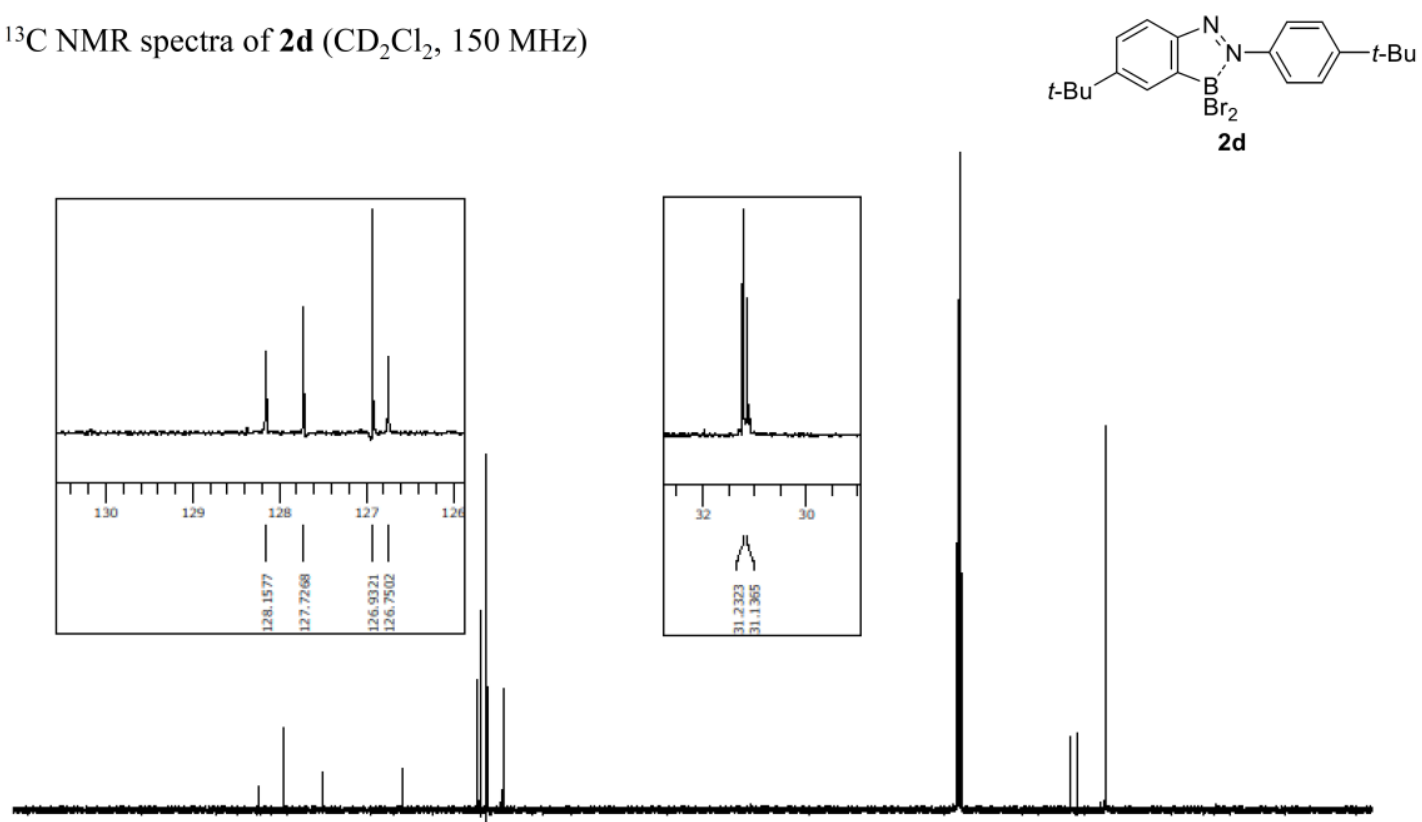

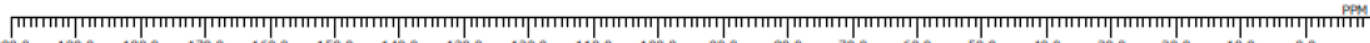

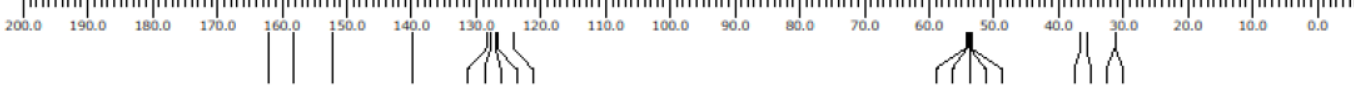

${ }^{11} \mathrm{~B}$ NMR spectra of $\mathbf{2 d}\left(\mathrm{CD}_{2} \mathrm{Cl}_{2}, 193 \mathrm{MHz}\right)$<smiles>CC(C)(C)c1ccc(N2[B]c3cc(C(C)(C)C)ccc3N=N2)cc1</smiles>

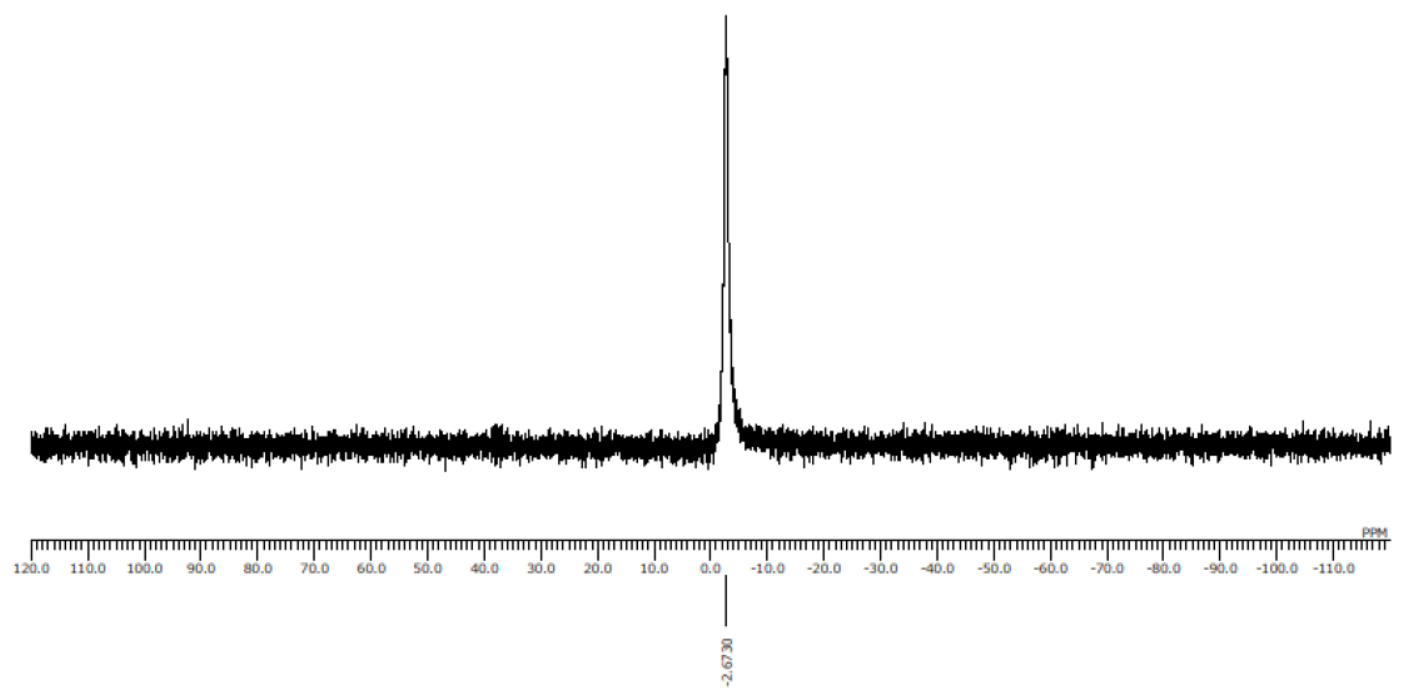


${ }^{1} \mathrm{H}$ NMR spectra of $\mathbf{2 e}\left(\mathrm{CD}_{2} \mathrm{Cl}_{2}, 600 \mathrm{MHz}\right)$
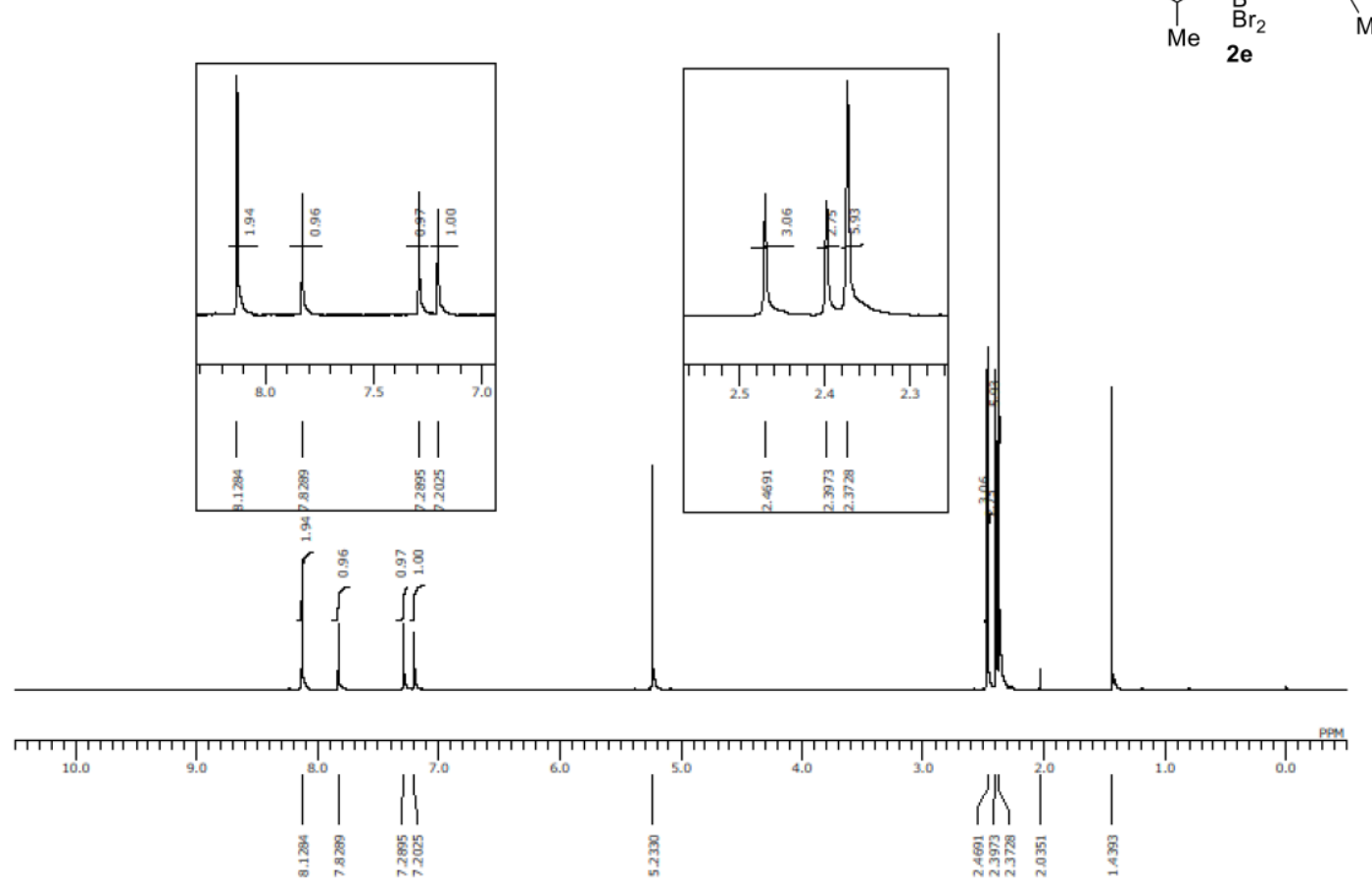

${ }^{13} \mathrm{C}$ NMR spectra of $2 \mathbf{e}\left(\mathrm{CD}_{2} \mathrm{Cl}_{2}, 150 \mathrm{MHz}\right)$
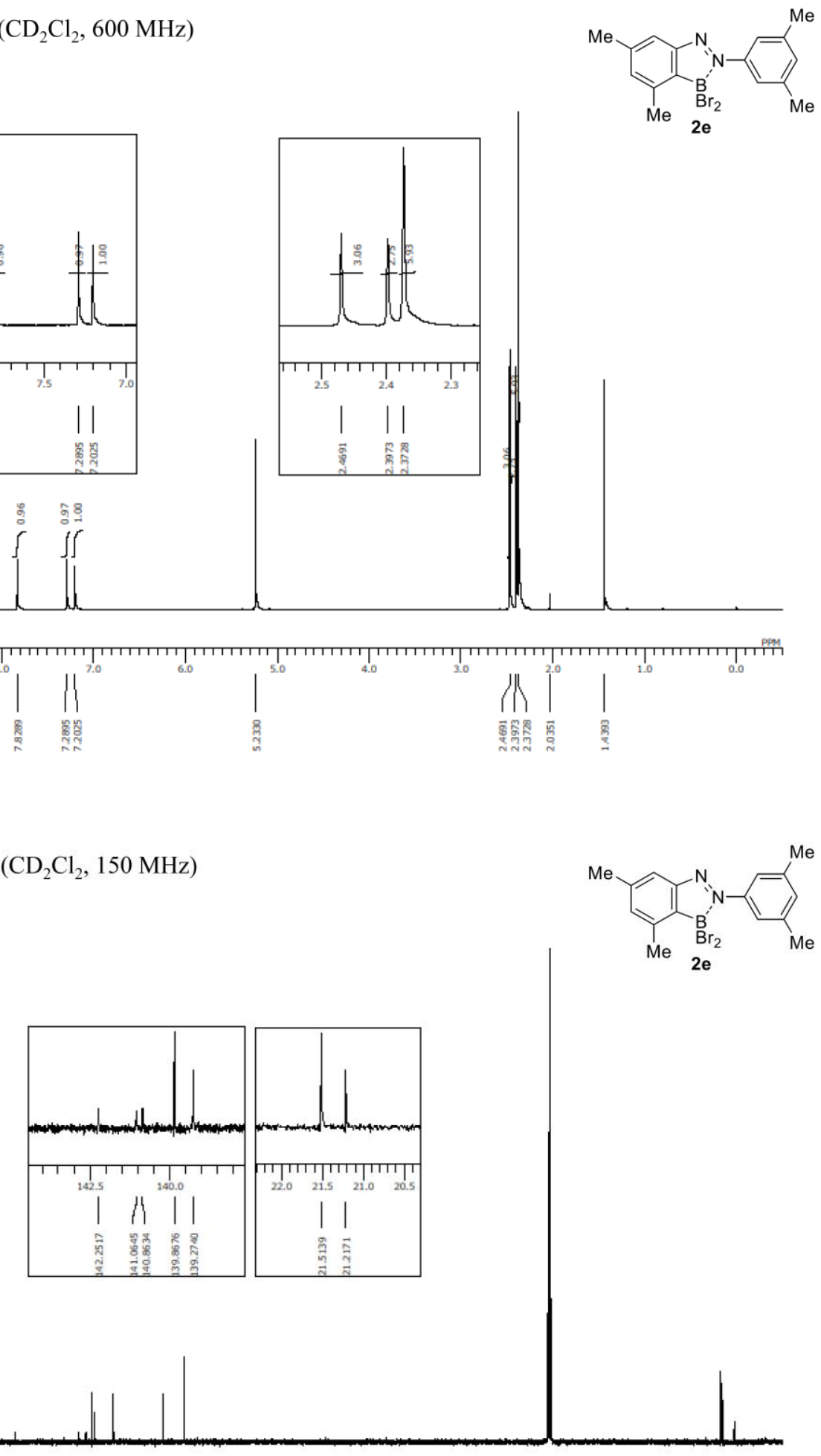

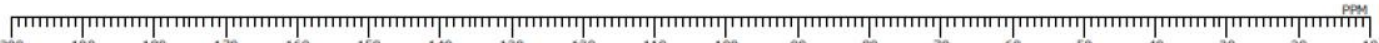

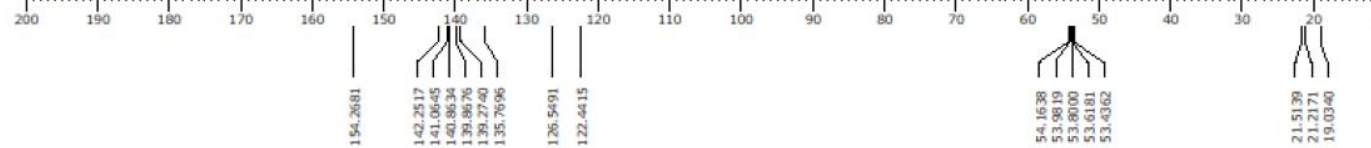


${ }^{11} \mathrm{~B}$ NMR spectra of $2 \mathrm{e}\left(\mathrm{CD}_{2} \mathrm{Cl}_{2}, 193 \mathrm{MHz}\right)$
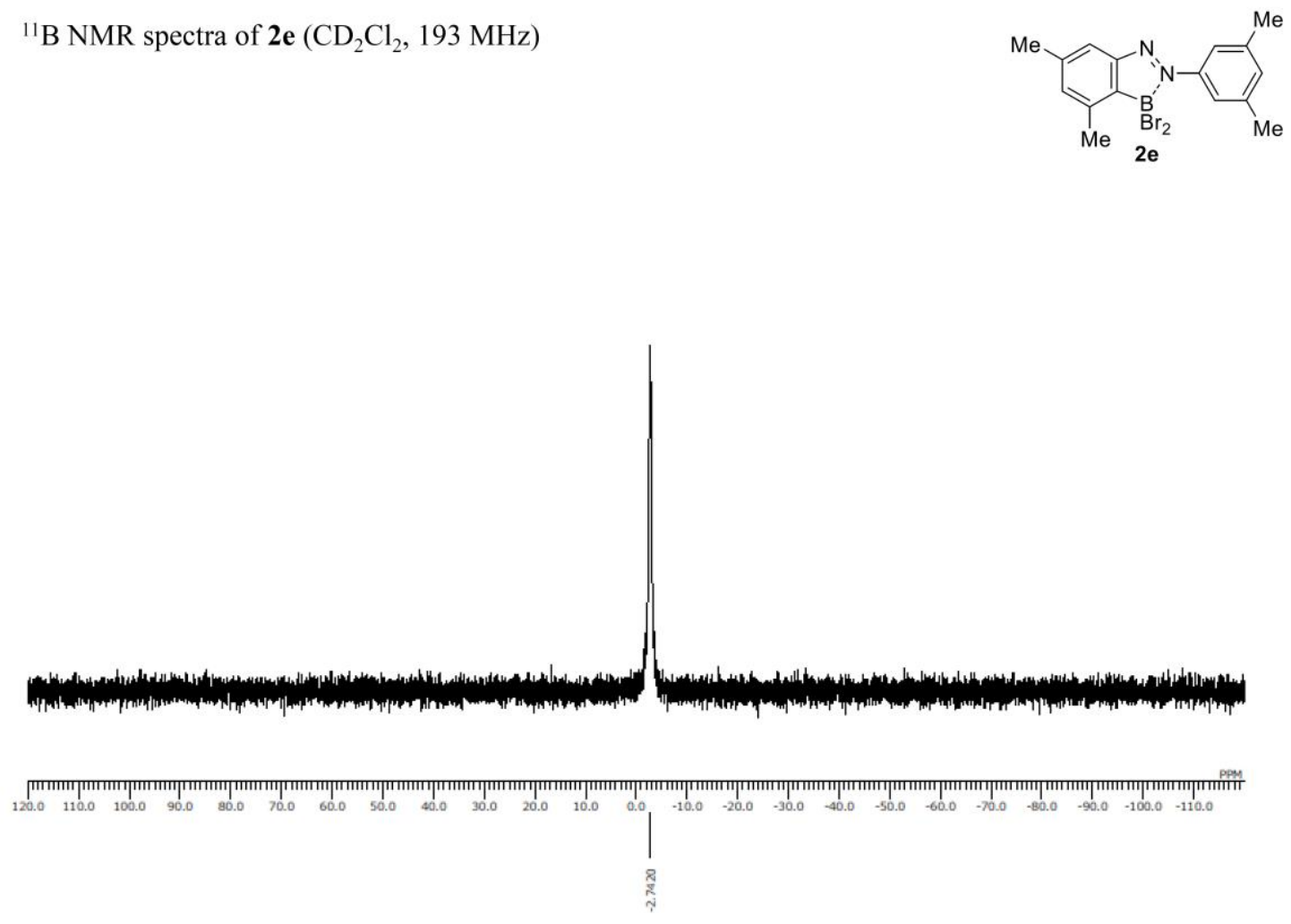

${ }^{1} \mathrm{H}$ NMR spectra of $\mathbf{2 f}\left(\mathrm{CD}_{2} \mathrm{Cl}_{2}, 600 \mathrm{MHz}\right)$
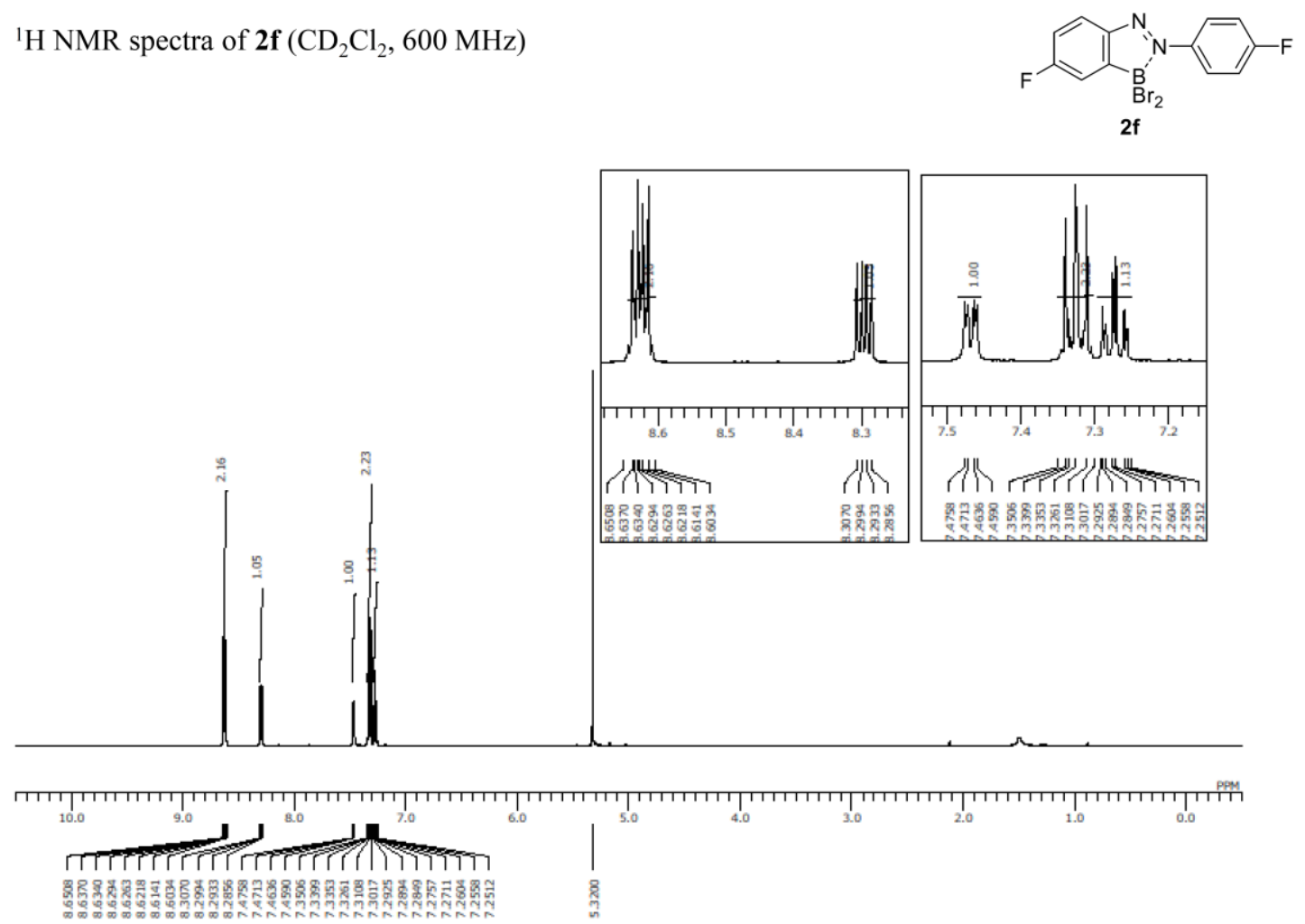
${ }^{13} \mathrm{C}$ NMR spectra of $\mathbf{2} \mathbf{f}\left(\mathrm{CD}_{2} \mathrm{Cl}_{2}, 150 \mathrm{MHz}\right)$

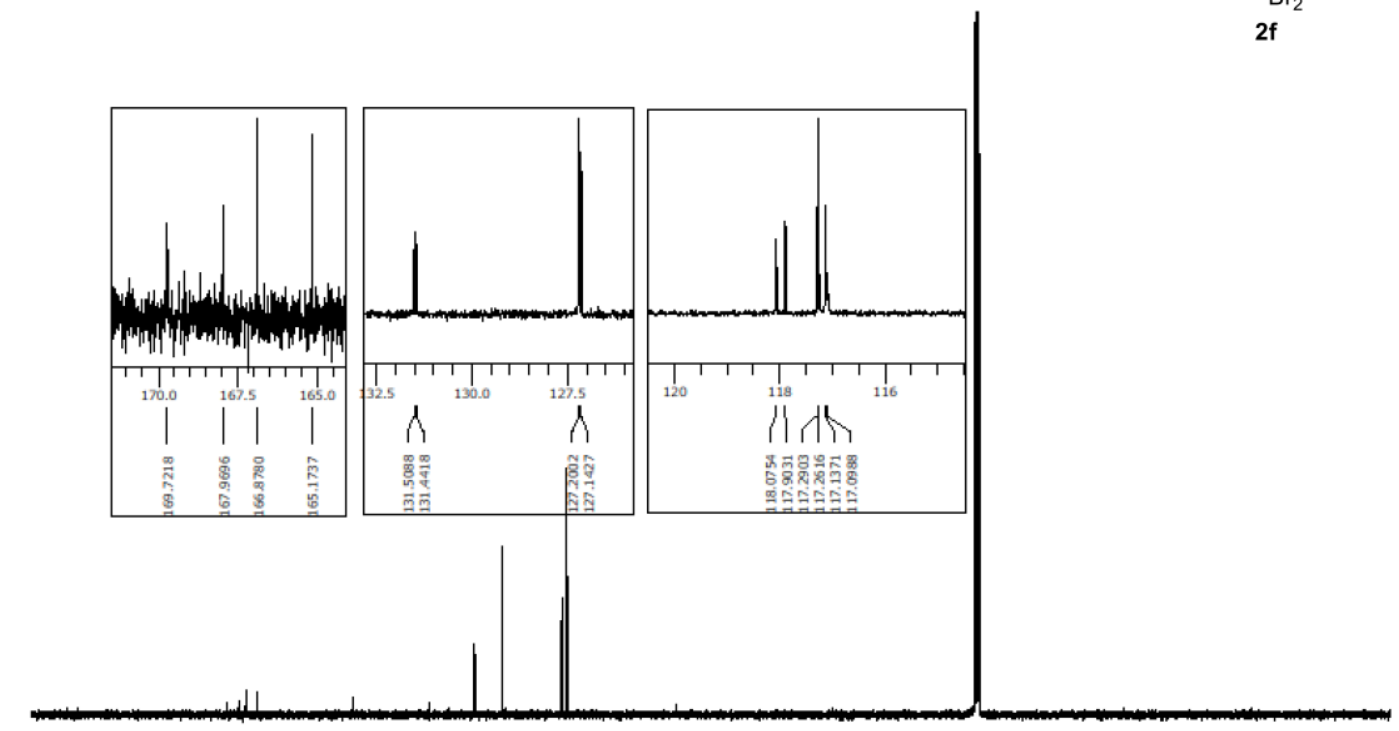

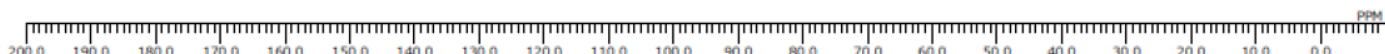

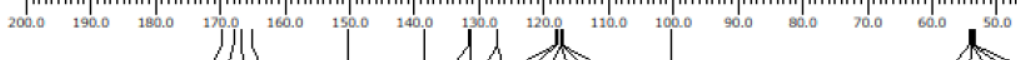

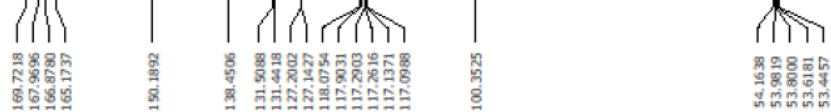

${ }^{11} \mathrm{~B}$ NMR spectra of $\mathbf{2} \mathbf{f}\left(\mathrm{CD}_{2} \mathrm{Cl}_{2}, 193 \mathrm{MHz}\right)$

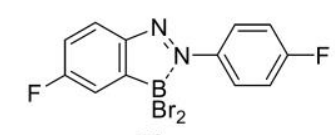

$2 f$

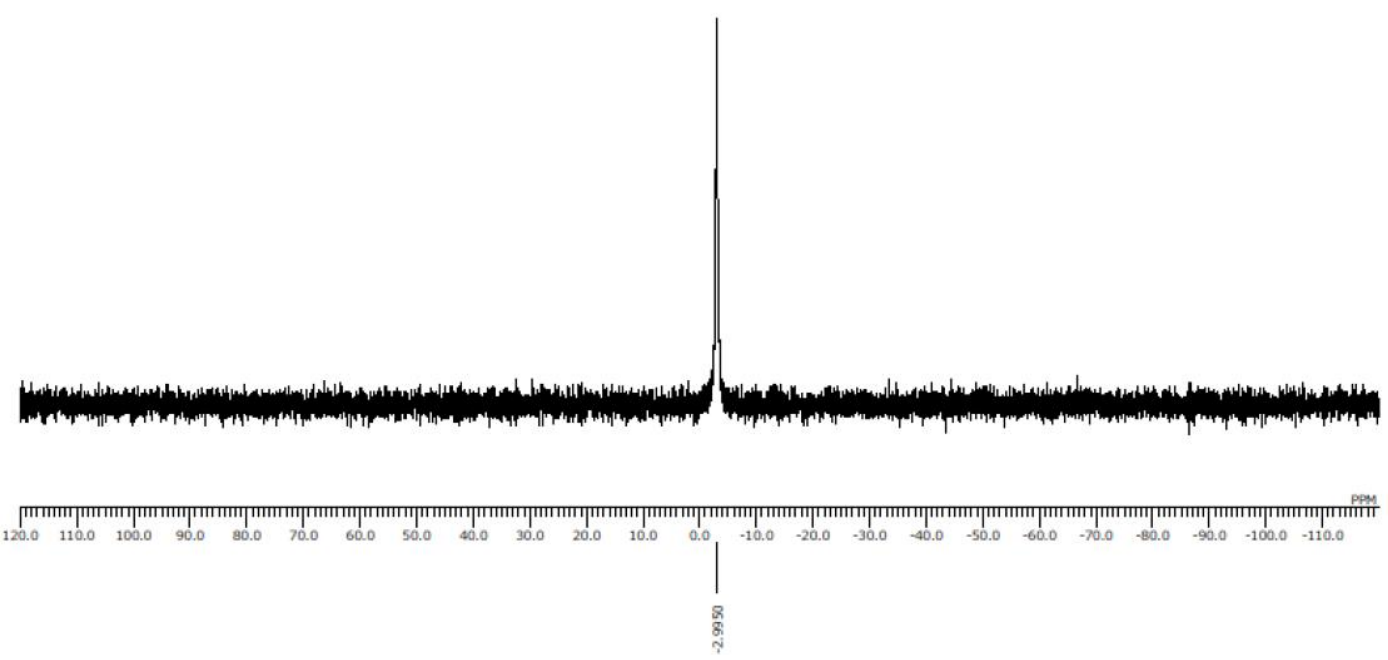


${ }^{1} \mathrm{H}$ NMR spectra of $\mathbf{2 g}\left(\mathrm{CD}_{2} \mathrm{Cl}_{2}, 600 \mathrm{MHz}\right)$
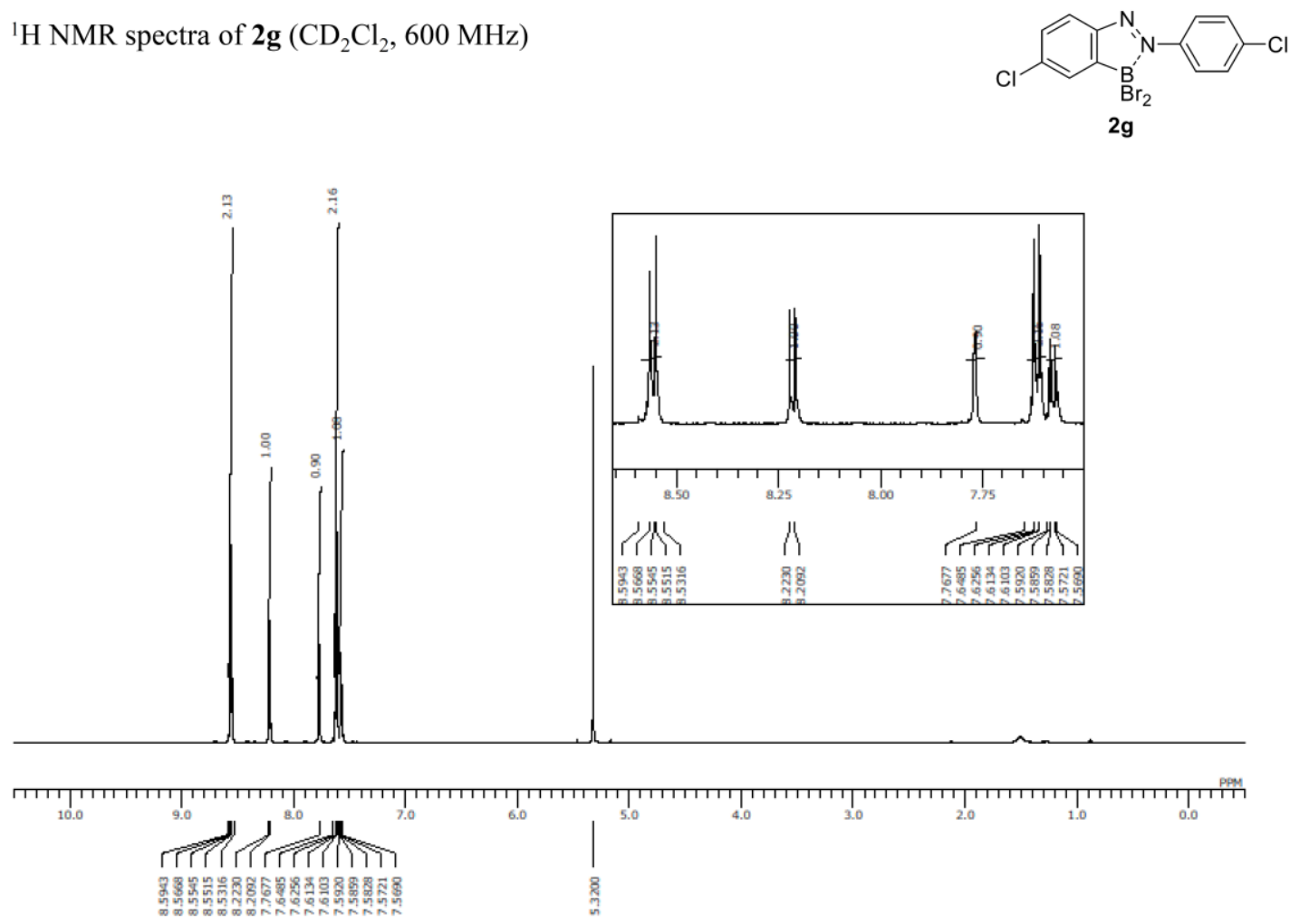

${ }^{13} \mathrm{C}$ NMR spectra of $\mathbf{2 g}\left(\mathrm{CD}_{2} \mathrm{Cl}_{2}, 150 \mathrm{MHz}\right)$

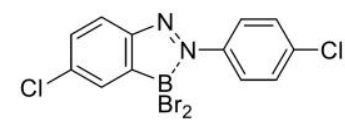

2g

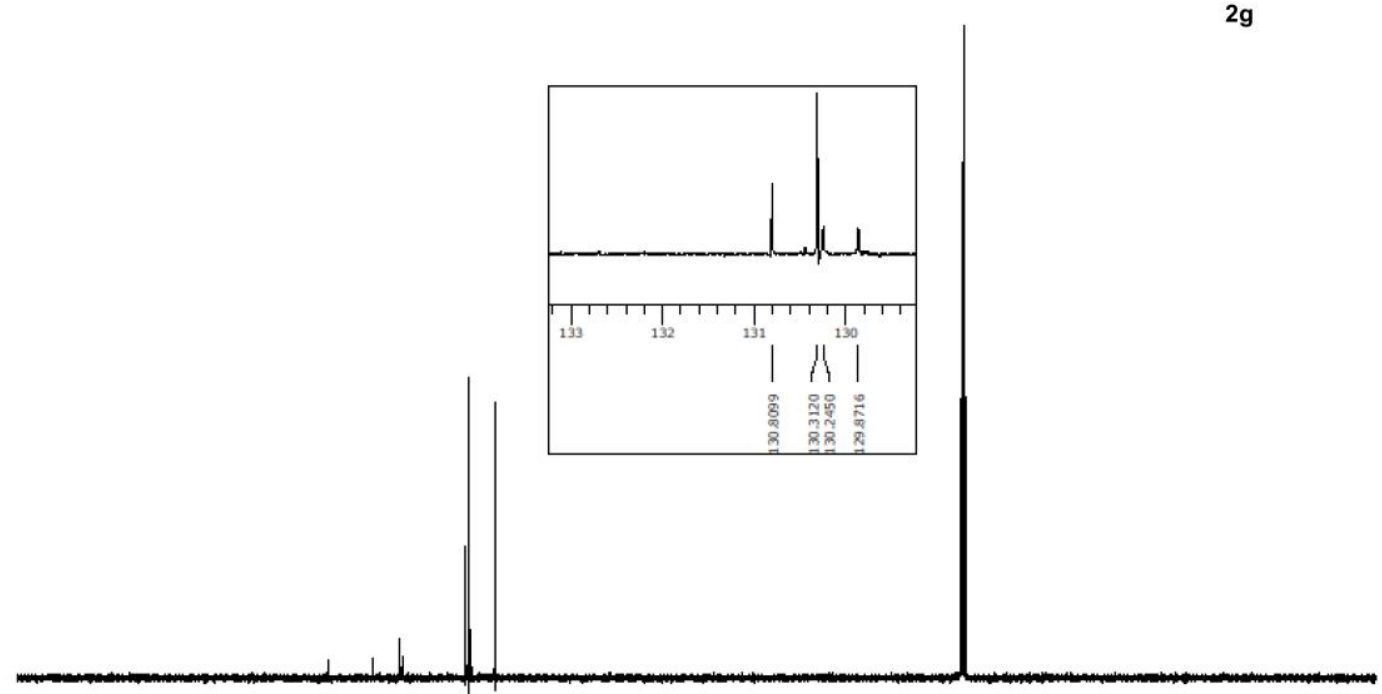

[

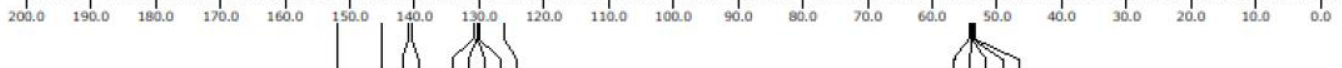

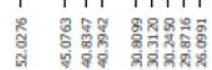

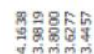


${ }^{11} \mathrm{~B}$ NMR spectra of $\mathbf{2} \mathbf{g}\left(\mathrm{CD}_{2} \mathrm{Cl}_{2}, 193 \mathrm{MHz}\right)$

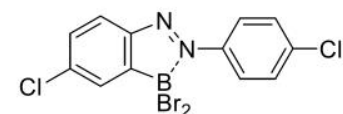

2g

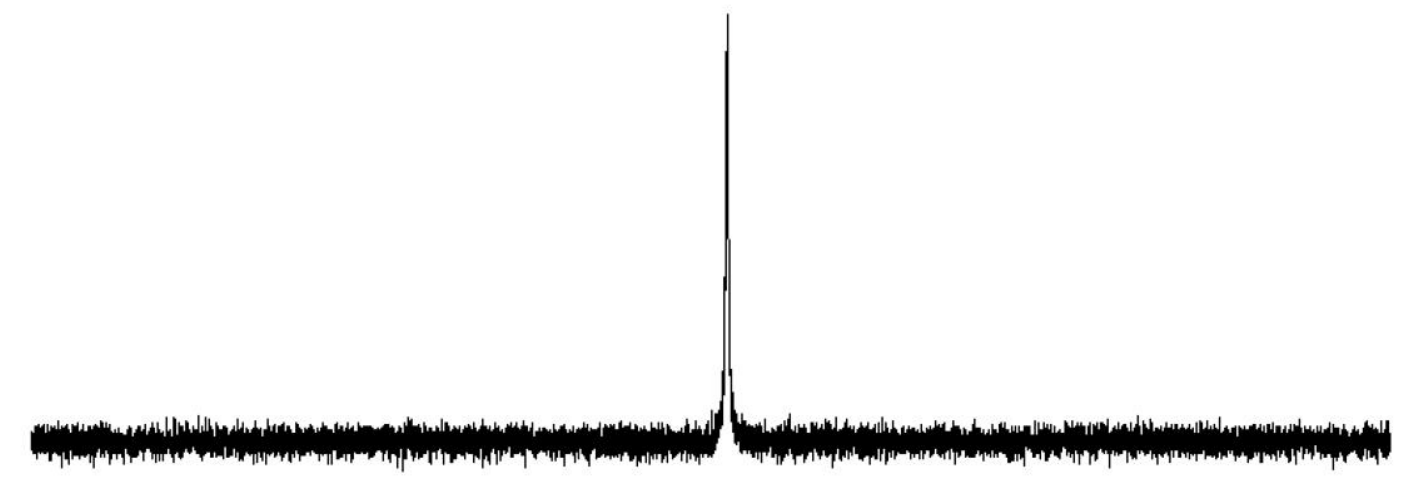

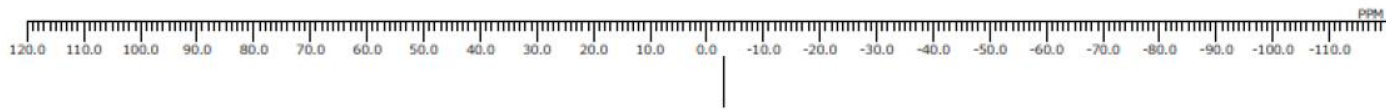

${ }^{1} \mathrm{H}$ NMR spectra of $\mathbf{2 h}\left(\mathrm{CD}_{2} \mathrm{Cl}_{2}, 600 \mathrm{MHz}\right)$

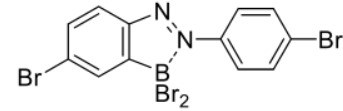

$2 \mathrm{~h}$

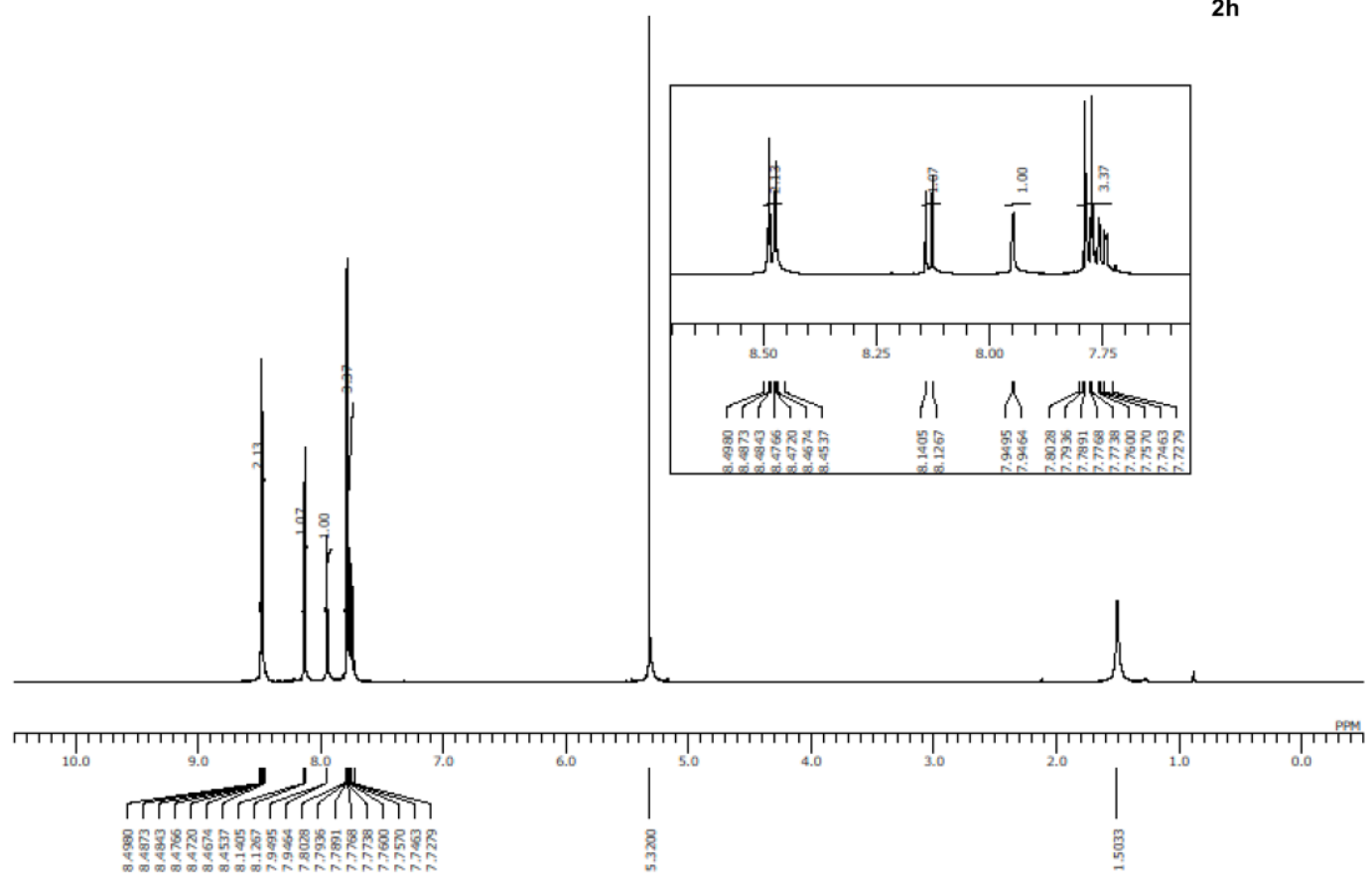


${ }^{13} \mathrm{C}$ NMR spectra of $\mathbf{2 h}\left(\mathrm{CD}_{2} \mathrm{Cl}_{2}, 150 \mathrm{MHz}\right)$

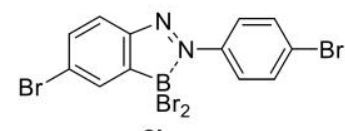

2h
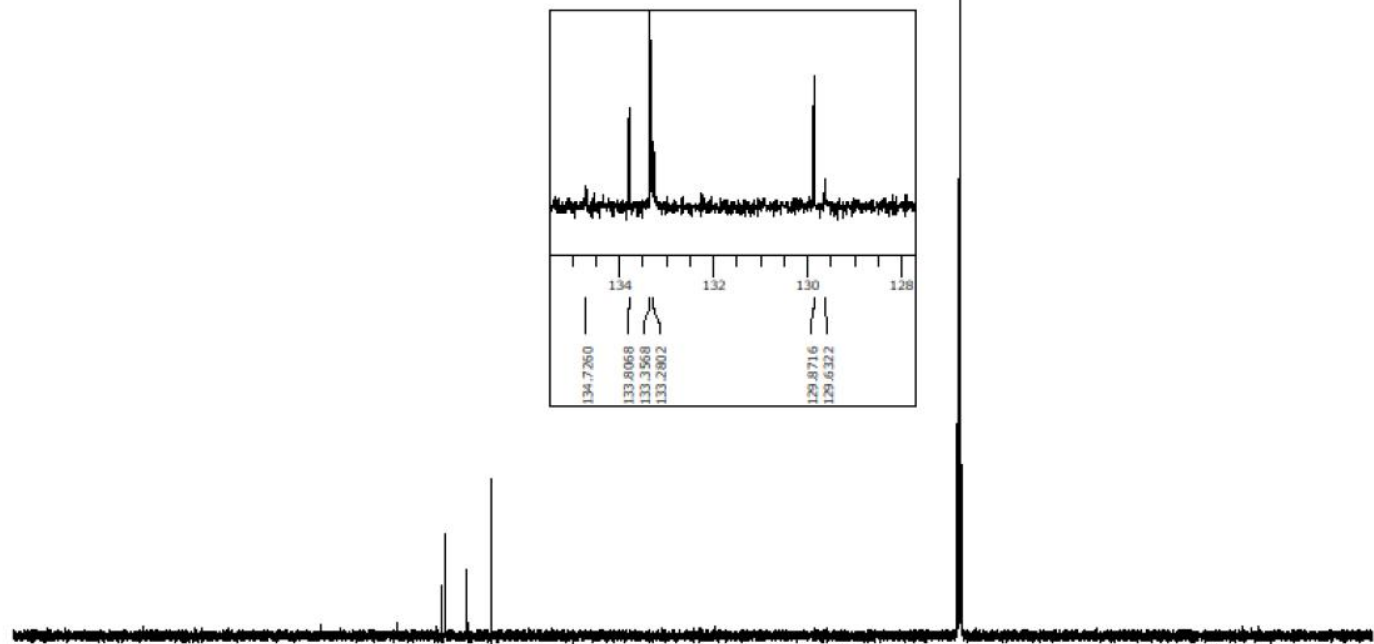

[

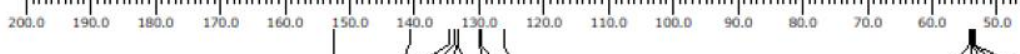

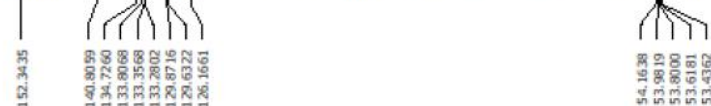

${ }^{11} \mathrm{~B}$ NMR spectra of $\mathbf{2 h}\left(\mathrm{CD}_{2} \mathrm{Cl}_{2}, 193 \mathrm{MHz}\right)$

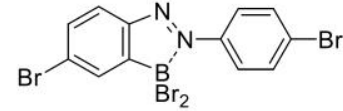

2h

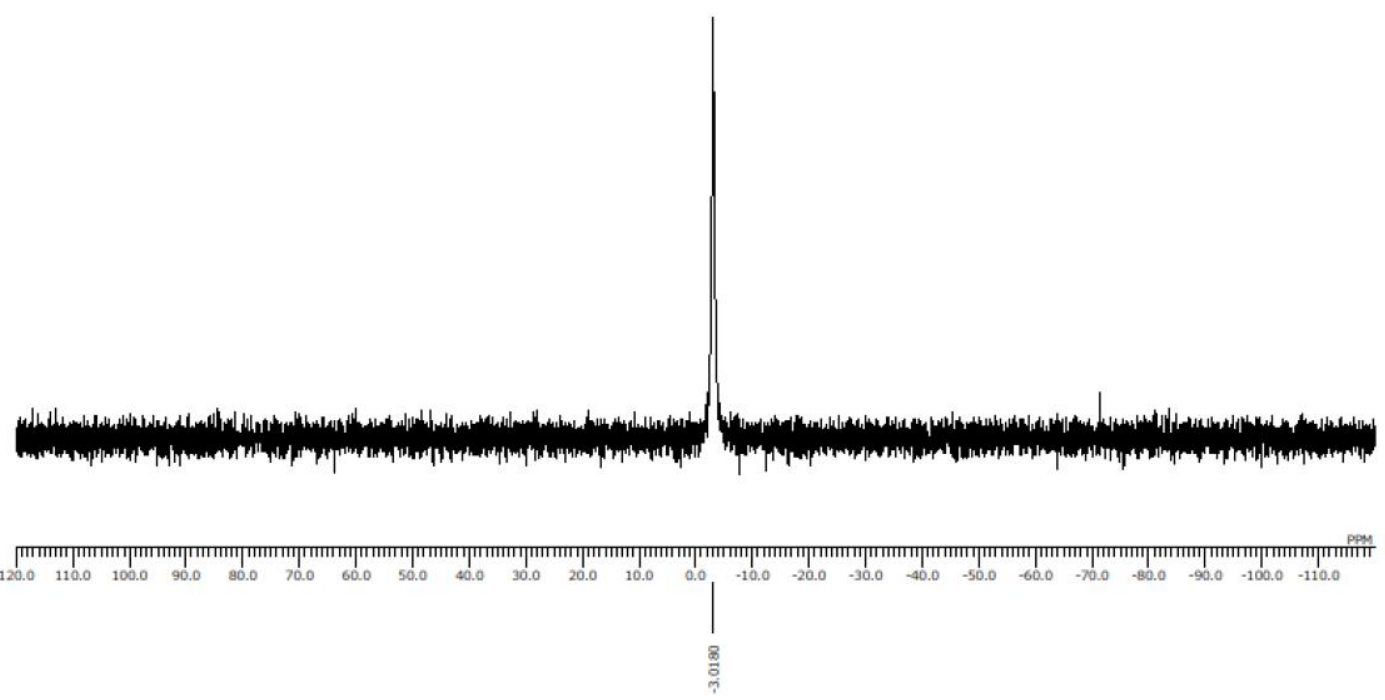


${ }^{1} \mathrm{H}$ NMR spectra of $\mathbf{2} \mathbf{i}\left(\mathrm{CDCl}_{3}, 600 \mathrm{MHz}\right)$

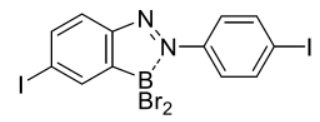

2i

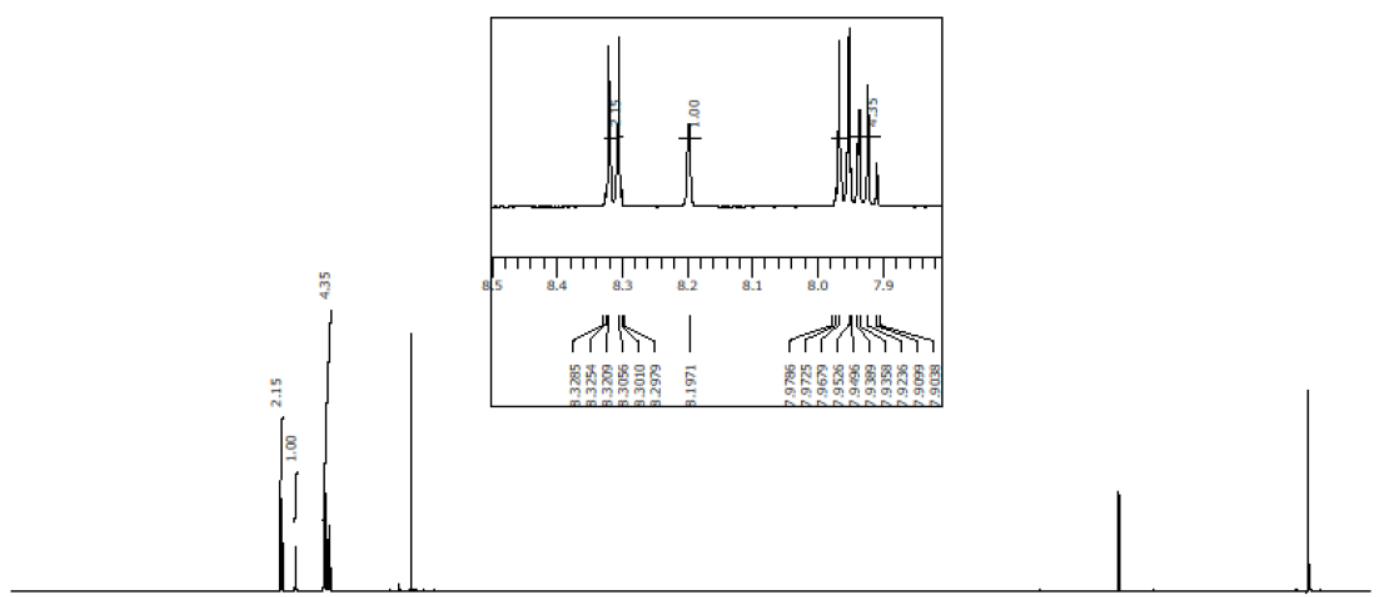

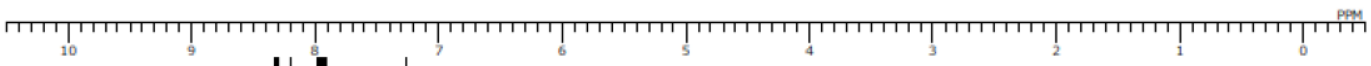
17\%?

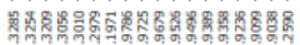

${ }^{13} \mathrm{C}$ NMR spectra of $\mathbf{2} \mathbf{i}\left(\mathrm{CDCl}_{3}, 150 \mathrm{MHz}\right)$
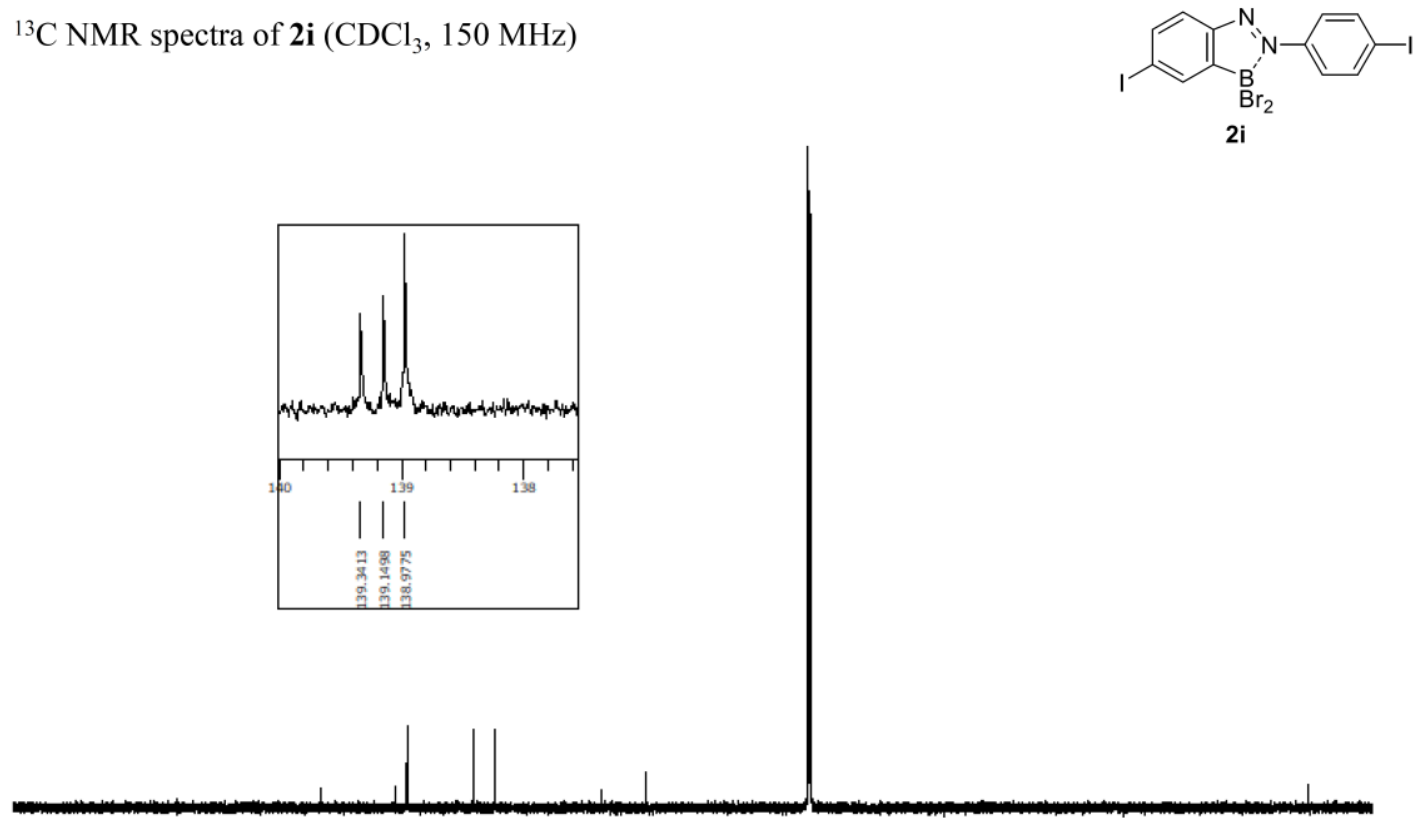

[

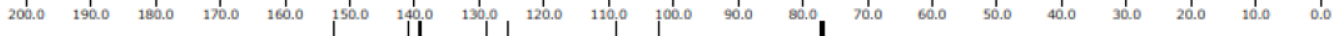

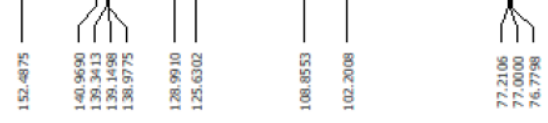


${ }^{11} \mathrm{~B}$ NMR spectra of $\mathbf{2 i}\left(\mathrm{CDCl}_{3}, 193 \mathrm{MHz}\right)$

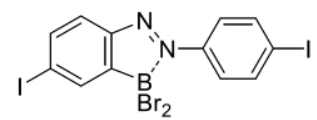

$2 \mathbf{i}$

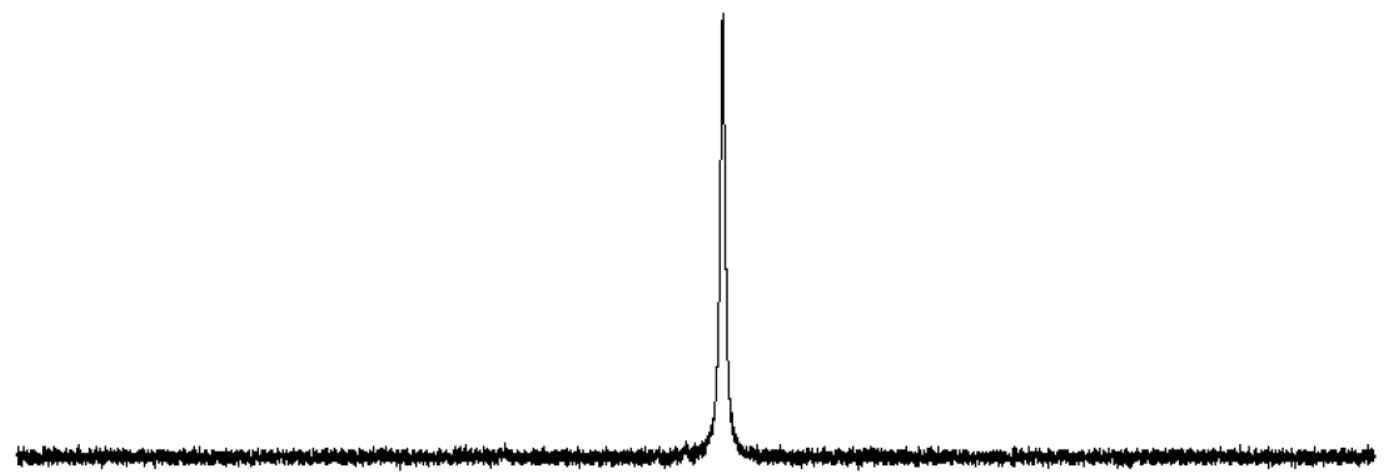

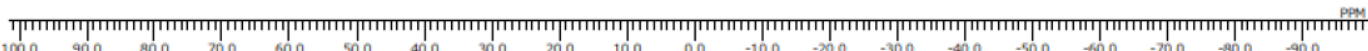

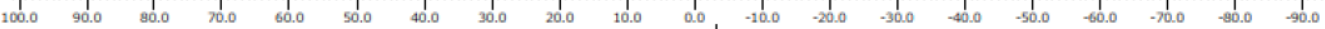

${ }^{1} \mathrm{H}$ NMR spectra of $\mathbf{2} \mathbf{j}\left(\mathrm{CD}_{2} \mathrm{Cl}_{2}, 600 \mathrm{MHz}\right)$

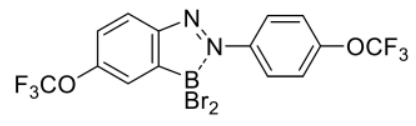

$2 \mathrm{j}$

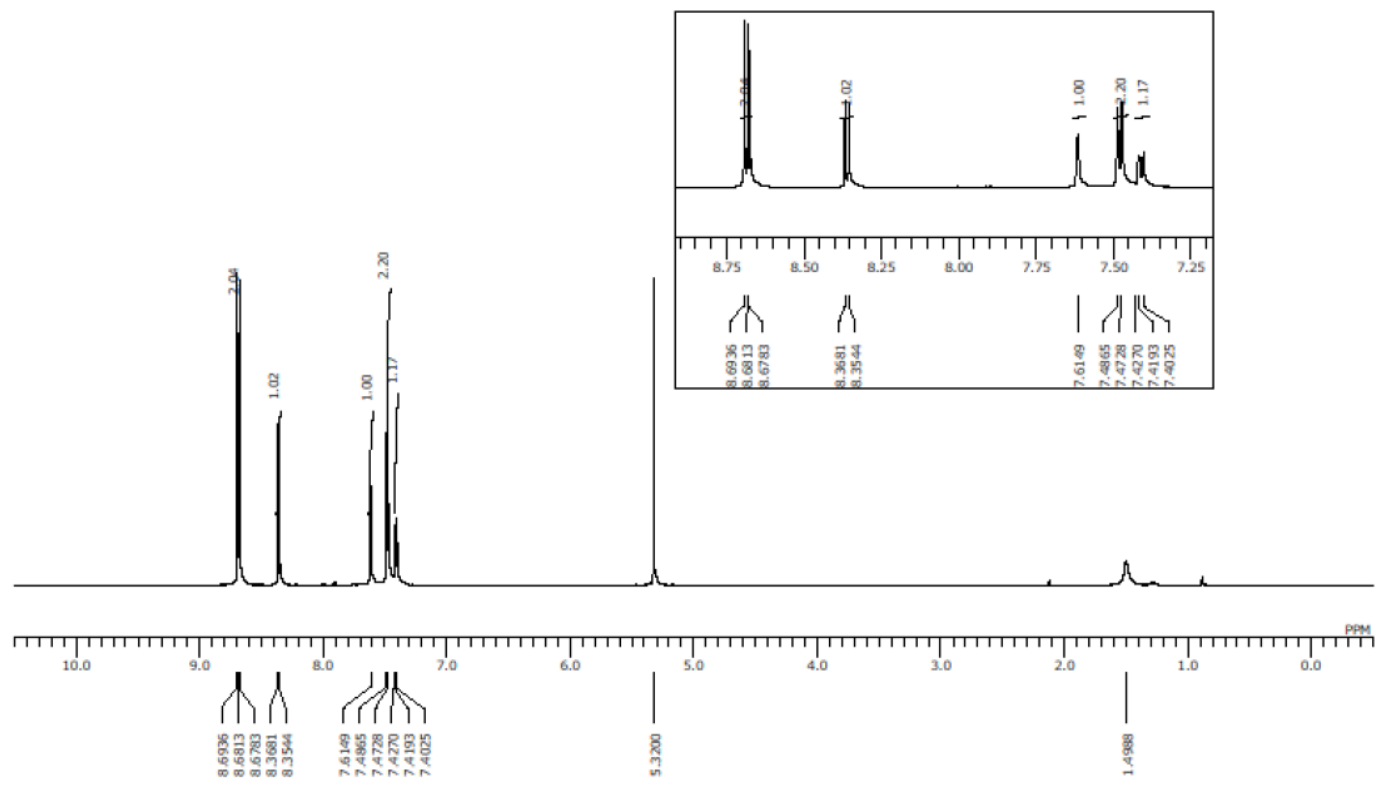


${ }^{13} \mathrm{C}$ NMR spectra of $\mathbf{2} \mathbf{j}\left(\mathrm{C}_{6} \mathrm{D}_{6}, 100 \mathrm{MHz}\right)$

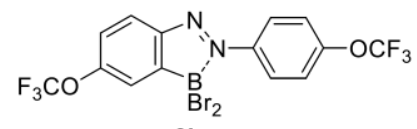

2j

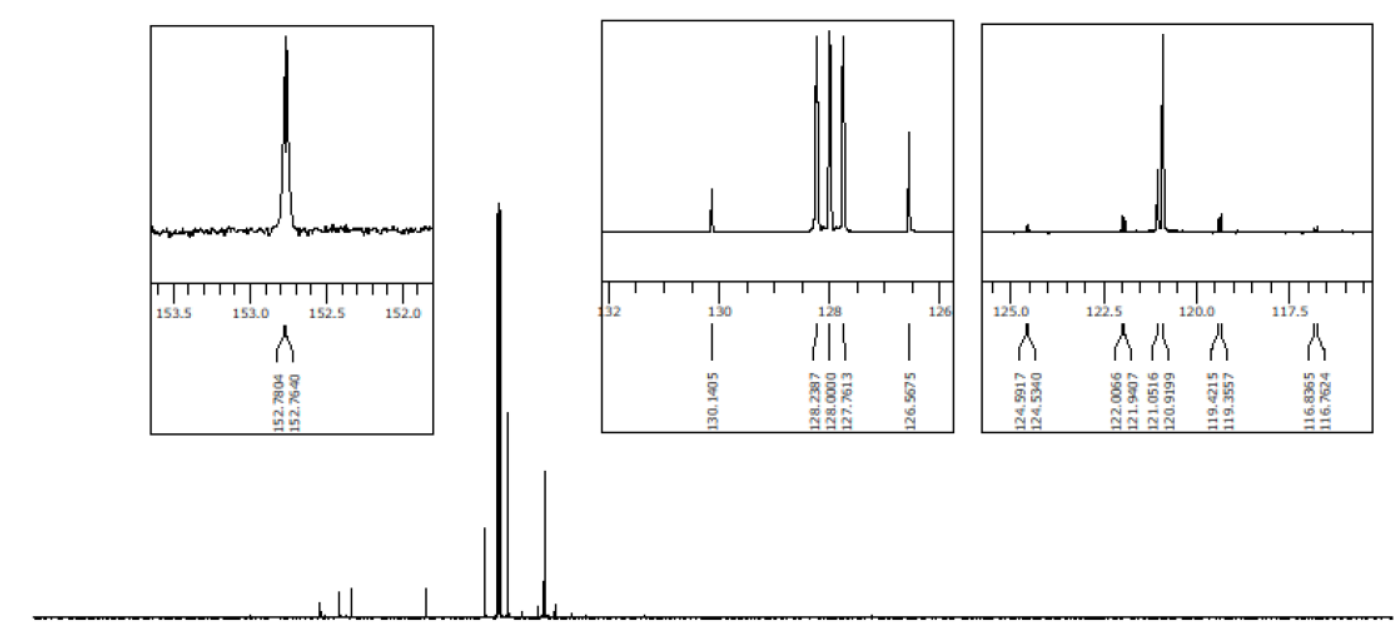

[

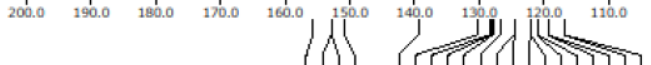

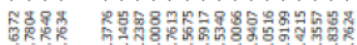

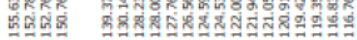

${ }^{11} \mathrm{~B}$ NMR spectra of $\mathbf{2} \mathbf{j}\left(\mathrm{CD}_{2} \mathrm{Cl}_{2}, 193 \mathrm{MHz}\right)$

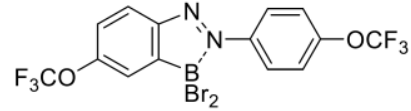

2j

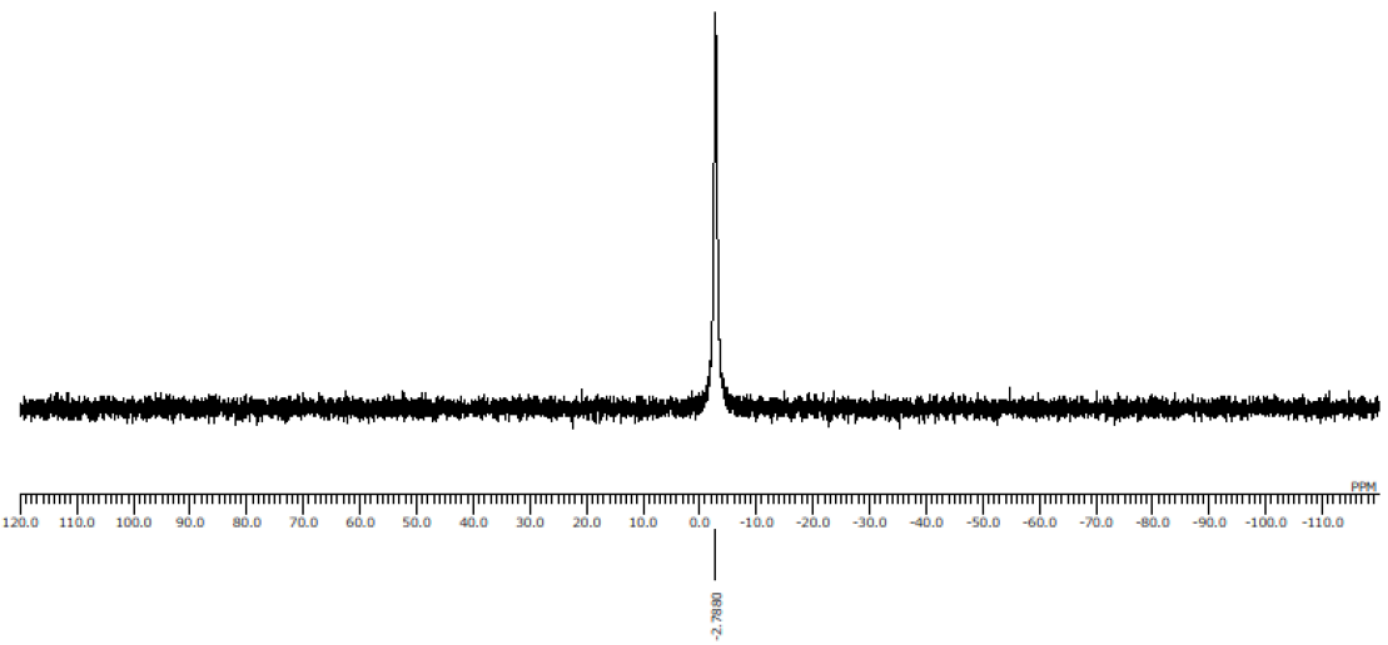


${ }^{1} \mathrm{H}$ NMR spectra of $\mathbf{2 k}\left(\mathrm{CDCl}_{3}, 400 \mathrm{MHz}\right)$
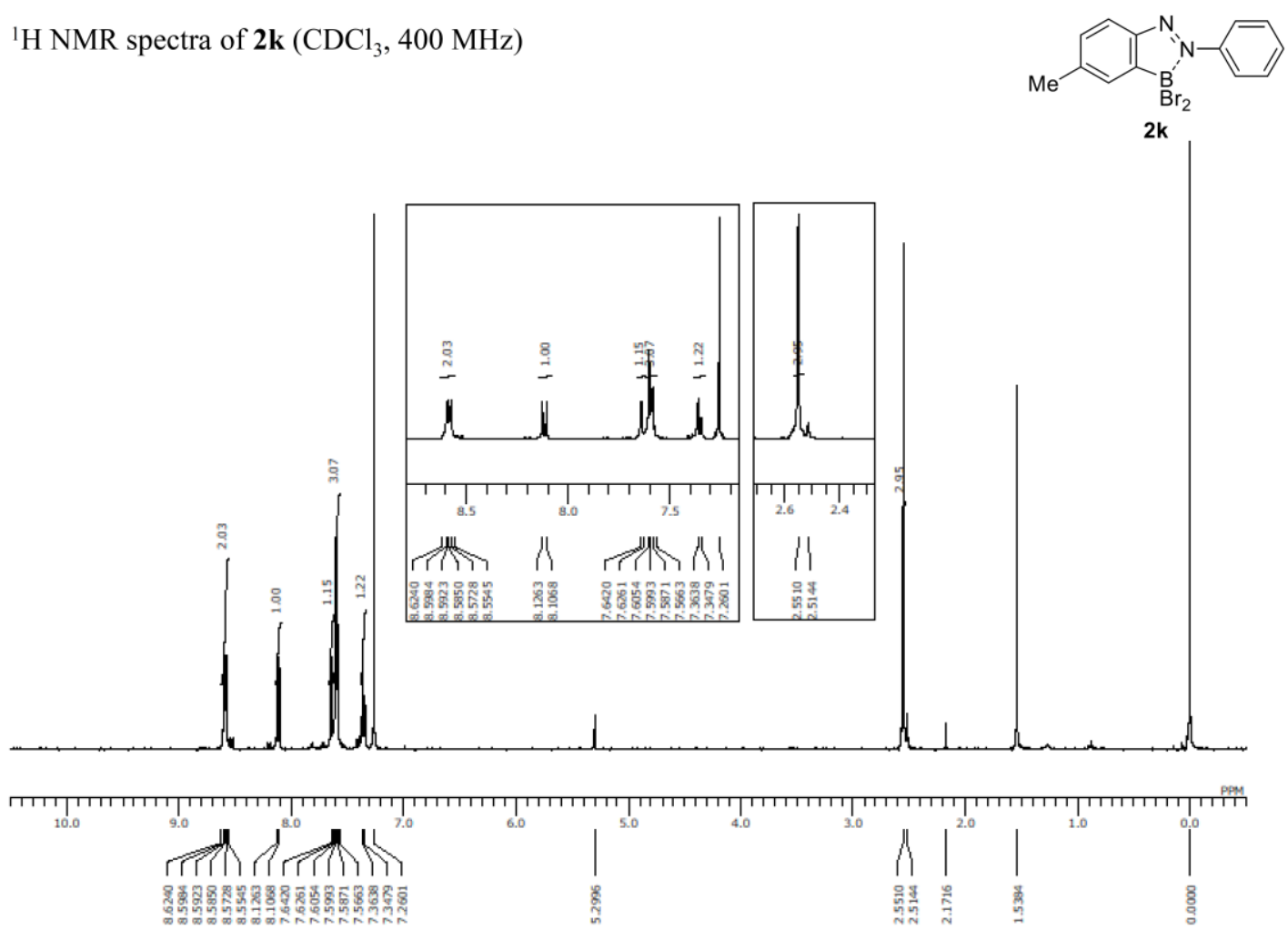

${ }^{13} \mathrm{C}$ NMR spectra of $\mathbf{2 k}\left(\mathrm{CDCl}_{3}, 150 \mathrm{MHz}\right)$
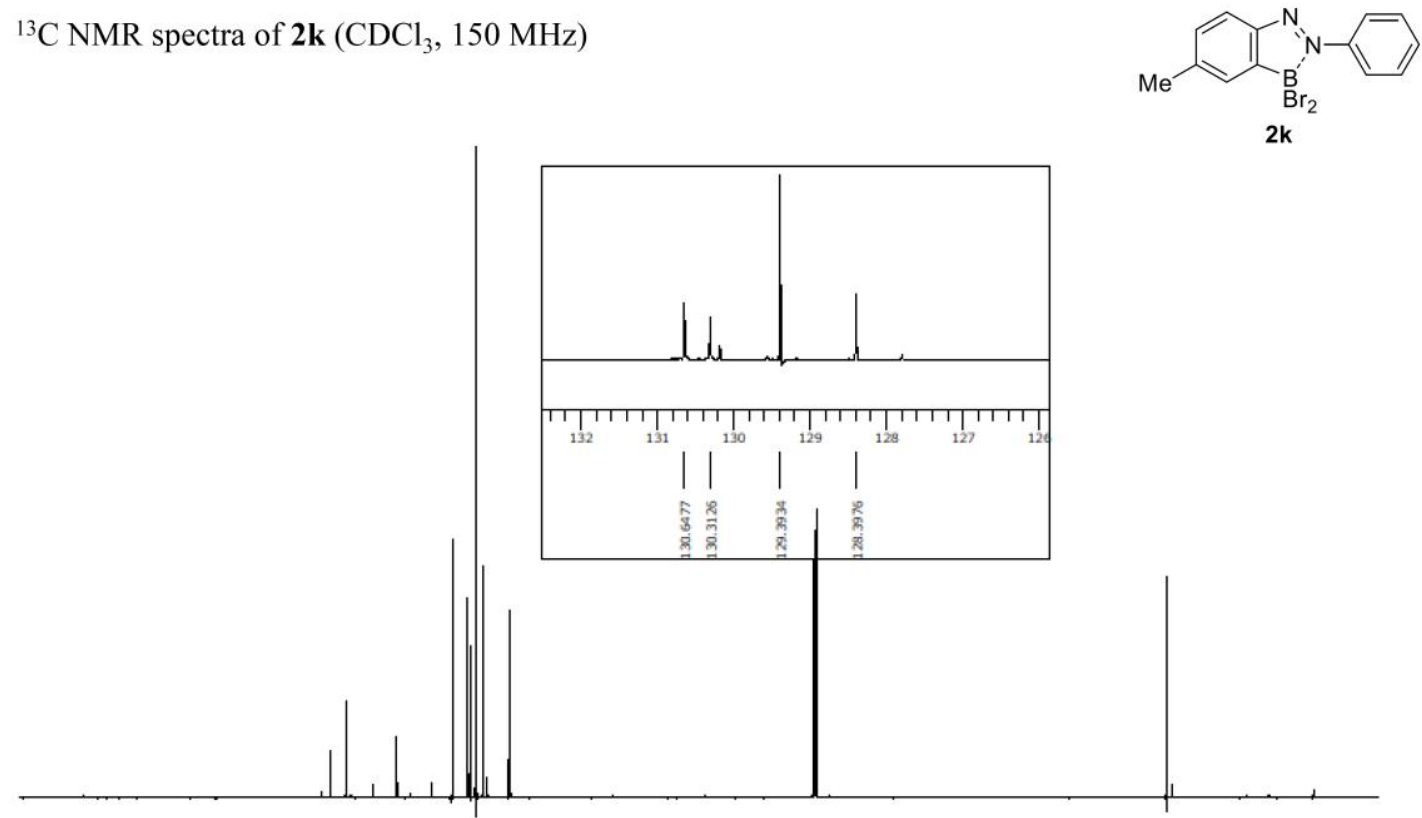

(

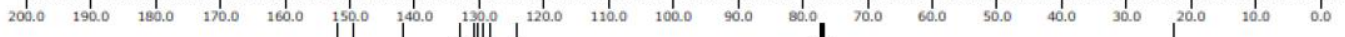
$\left.\left.200.0 \quad 190.0 \quad 180.0 \quad 170.0160 .0 \quad\right|^{150.0}\right|^{140.0} 130.0$

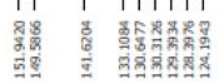

1)

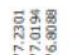


${ }^{11} \mathrm{~B}$ NMR spectra of $\mathbf{2 k}\left(\mathrm{CDCl}_{3}, 193 \mathrm{MHz}\right)$

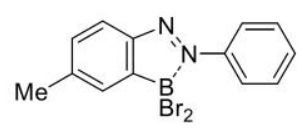

$2 k$

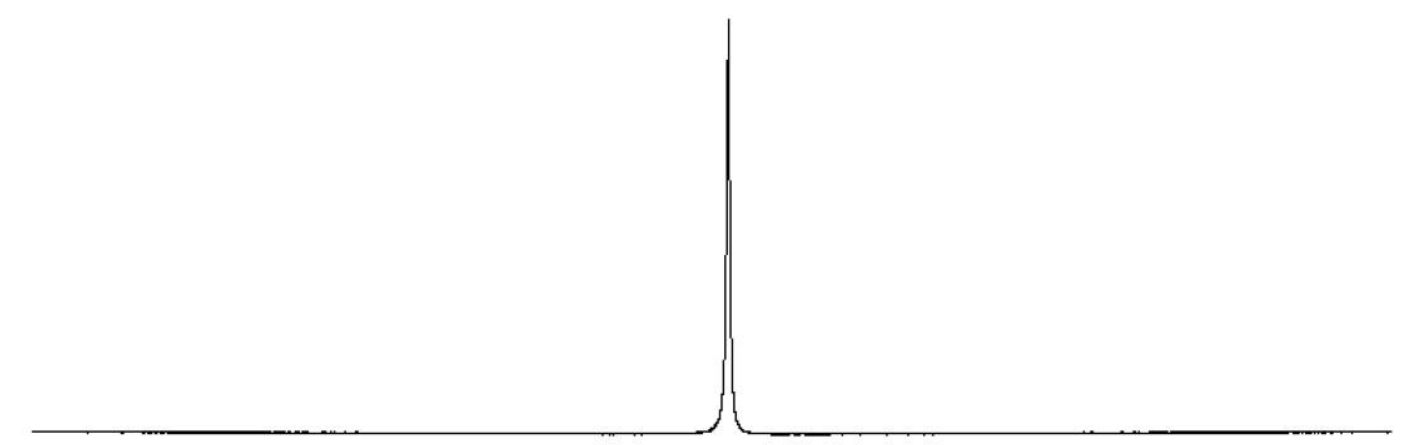

[ $\begin{array}{llllllllllllllllllllllll}120.0 & 110.0 & 100.0 & 90.0 & 90.0 & 70.0 & 60.0 & 50.0 & 40.0 & 30.0 & 20.0 & 10.0 & 0.0 & -10.0 & -20.0 & -30.0 & -40.0 & -50.0 & -60.0 & -70.0 & -80.0 & -90.0 & -100.0 & -110.0\end{array}$

${ }^{1} \mathrm{H}$ NMR spectra of $\mathbf{2 l}\left(\mathrm{CDCl}_{3}, 600 \mathrm{MHz}\right)$

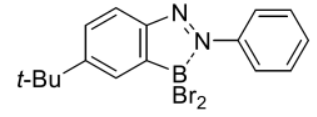

2I

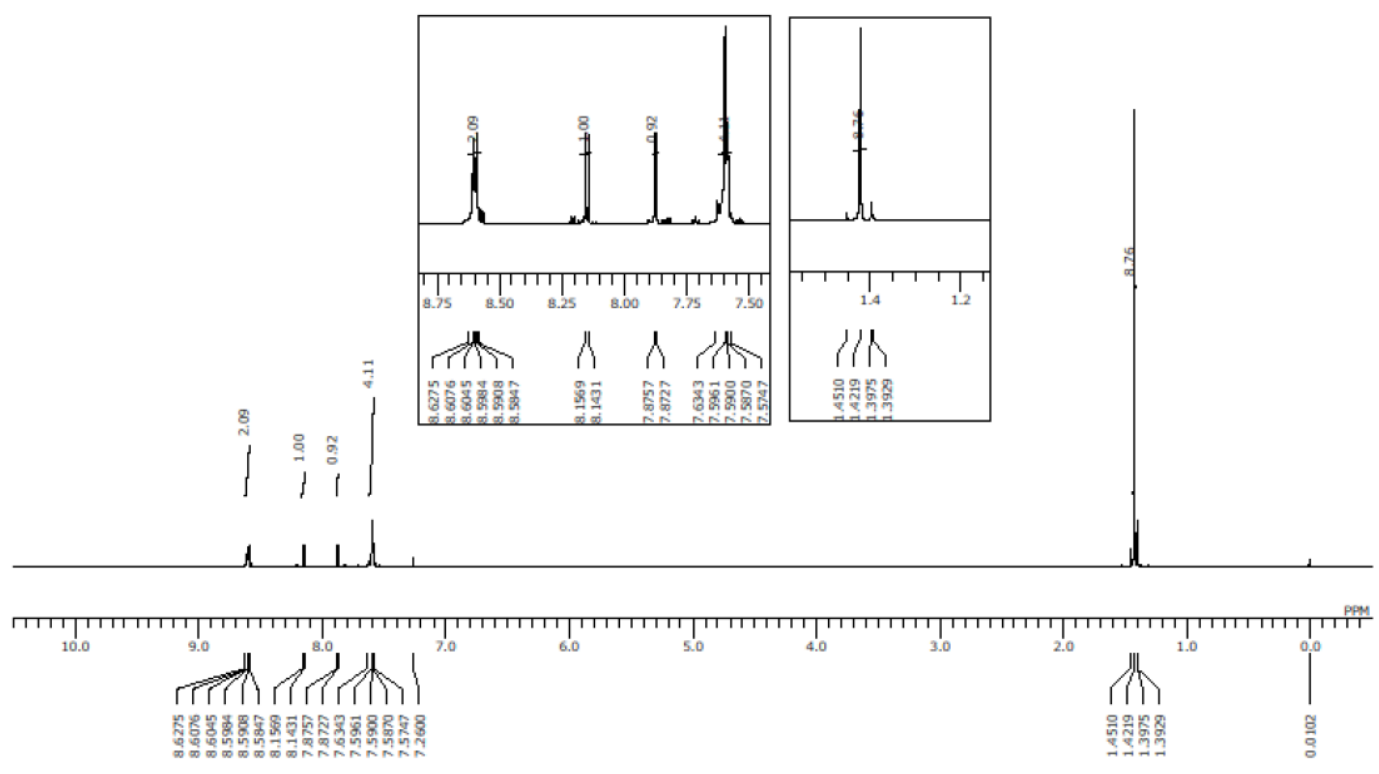


${ }^{13} \mathrm{C}$ NMR spectra of $2 \mathbf{l}\left(\mathrm{CDCl}_{3}, 150 \mathrm{MHz}\right)$
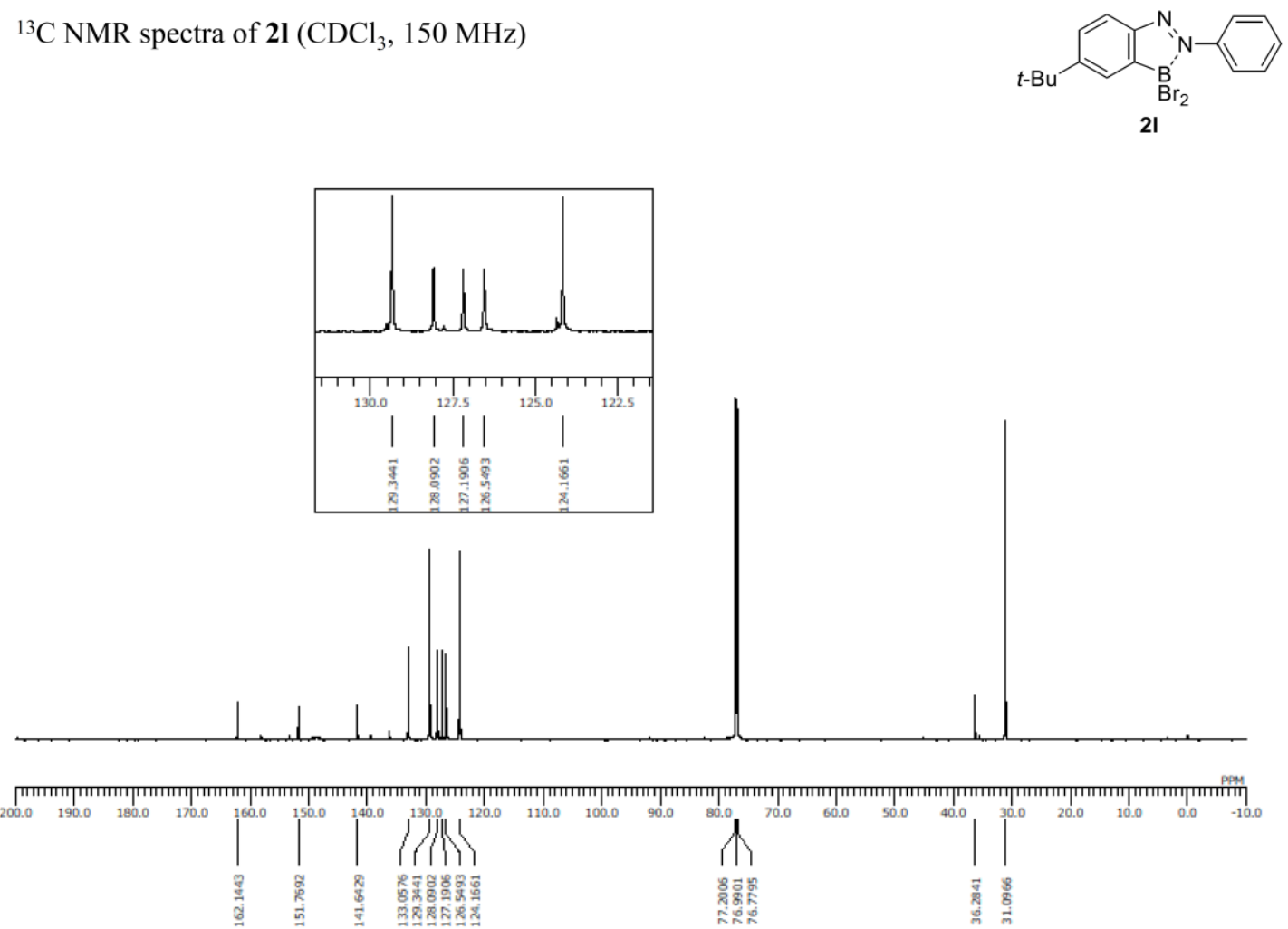

${ }^{11} \mathrm{~B}$ NMR spectra of $2 \mathbf{2}\left(\mathrm{CDCl}_{3}, 193 \mathrm{MHz}\right)$

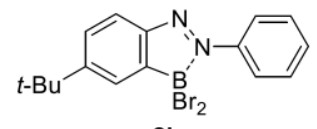

21

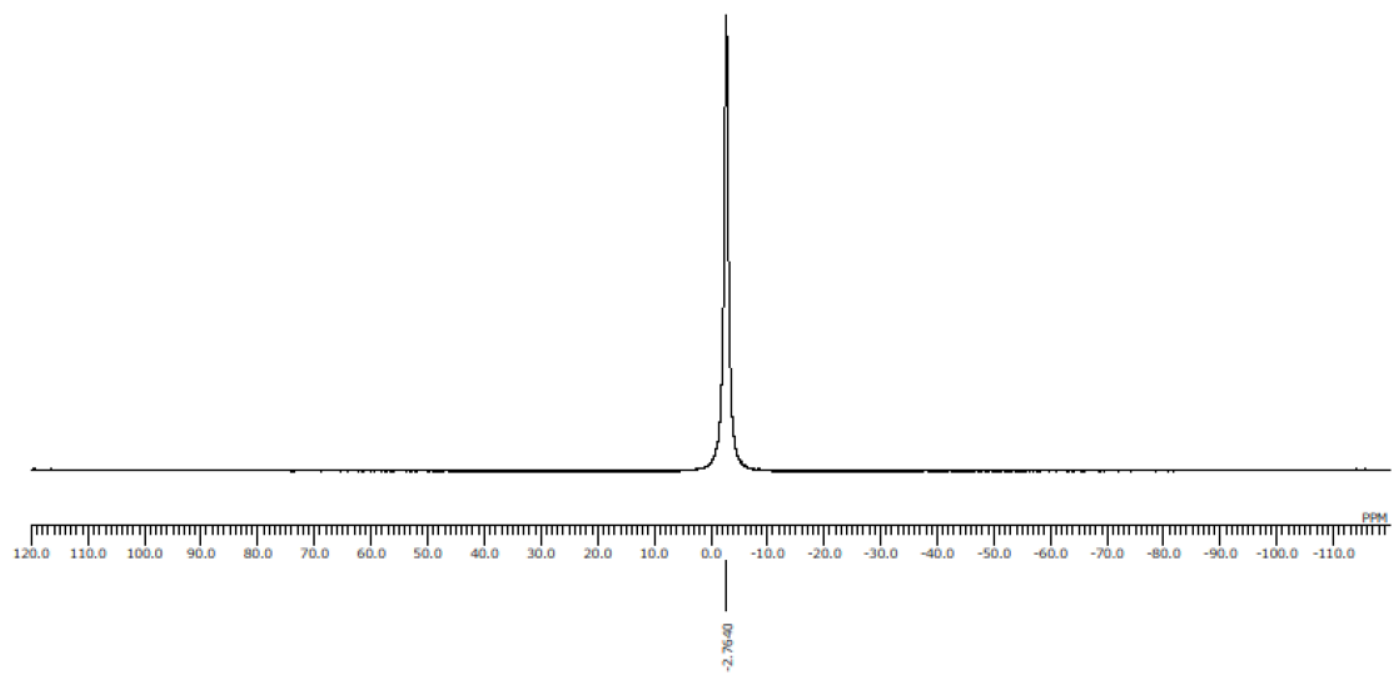


${ }^{1} \mathrm{H}$ NMR spectra of $\mathbf{1 3 a}\left(\mathrm{CDCl}_{3}, 400 \mathrm{MHz}\right)$

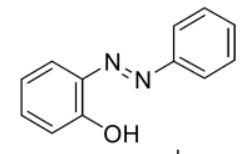

13

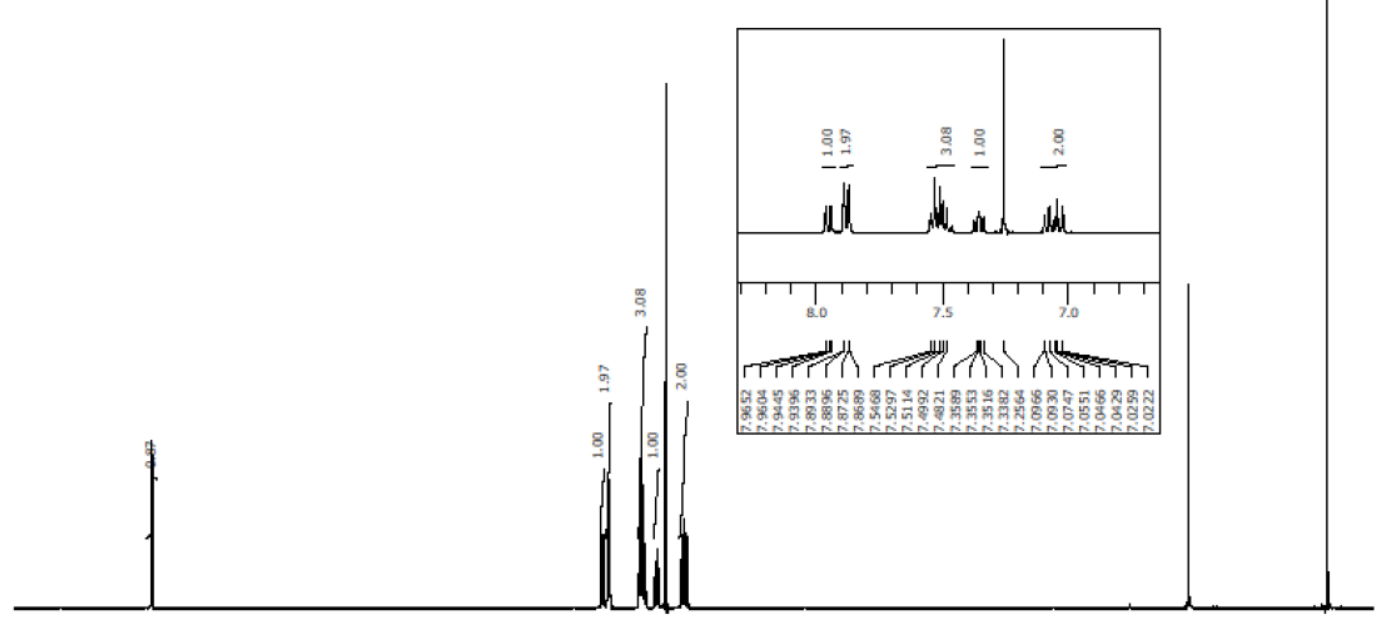

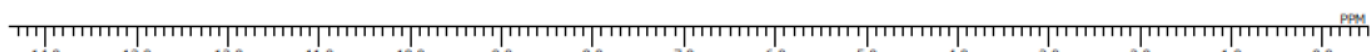

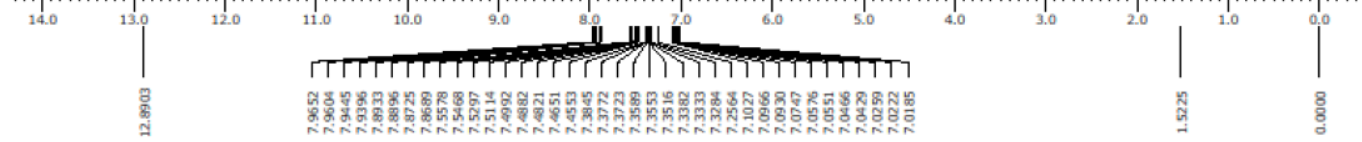

${ }^{13} \mathrm{C}$ NMR spectra of $13 \mathbf{a}\left(\mathrm{CDCl}_{3}, 150 \mathrm{MHz}\right)$
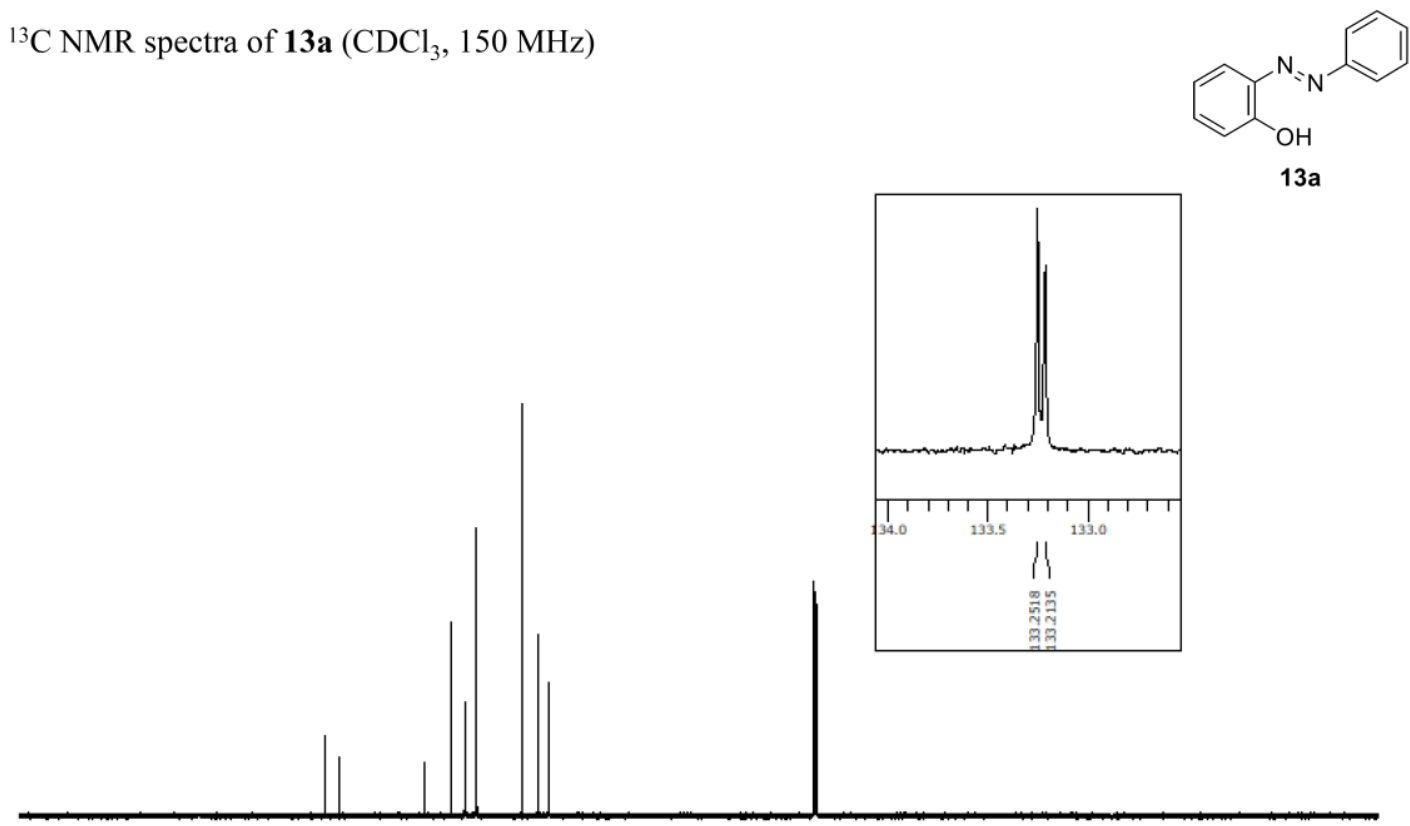

( $\begin{array}{llllllllllllll}200.0 & 190.0 & 100.0 & 170.0 & 160.0 & 150.0 & 140.0\end{array}$

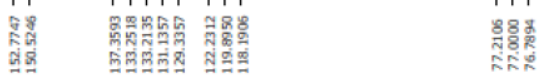


${ }^{1} \mathrm{H}$ NMR spectra of $14 \mathrm{~h}\left(\mathrm{DMSO}-d_{6}, 600 \mathrm{MHz}\right)$

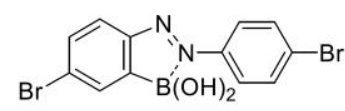

$14 \mathrm{~h}$
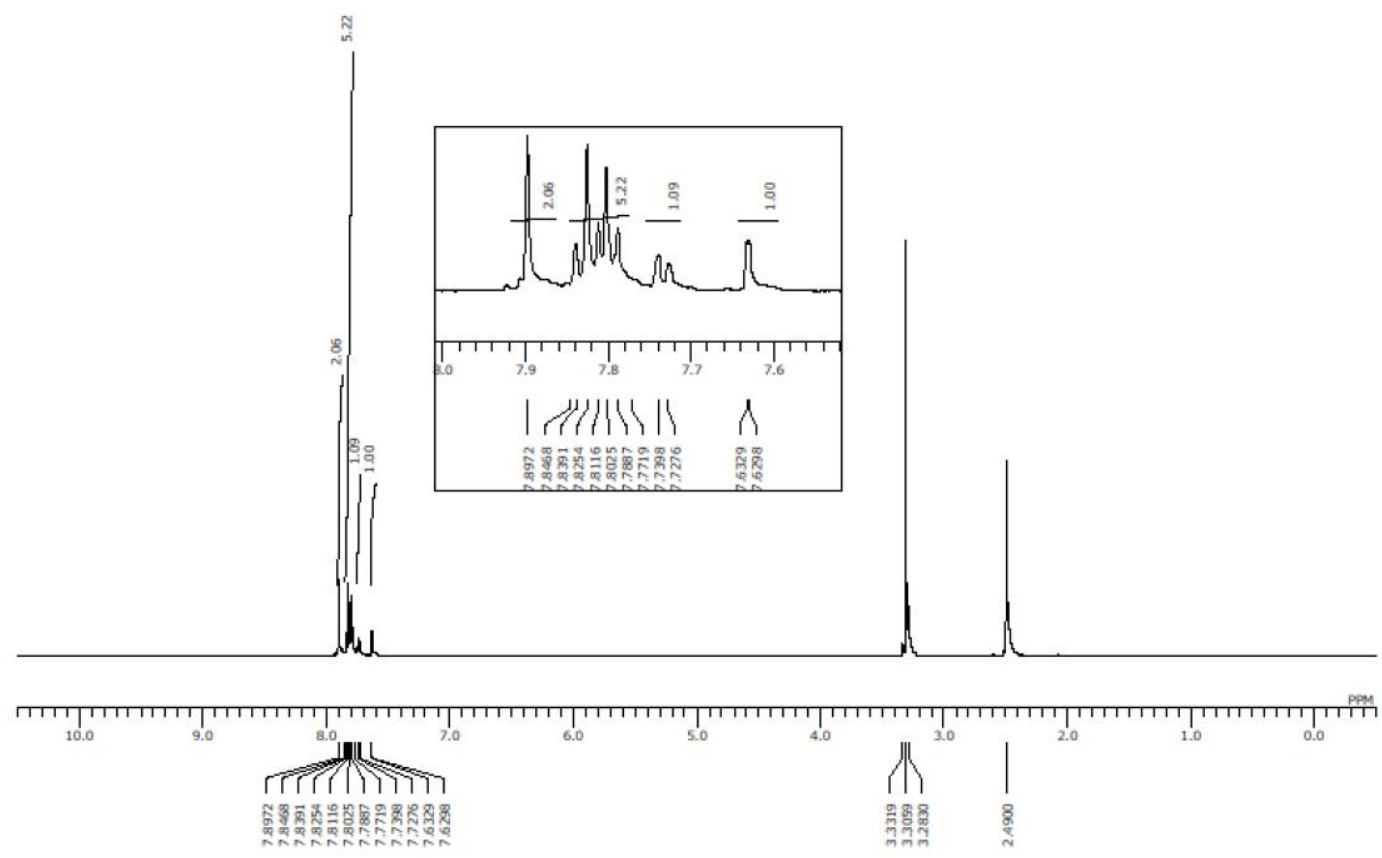

${ }^{13} \mathrm{C}$ NMR spectra of $\mathbf{1 4 h}\left(\mathrm{DMSO}-d_{6}, 150 \mathrm{MHz}\right)$

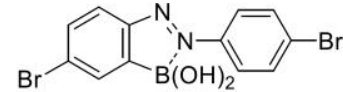

$14 \mathrm{~h}$
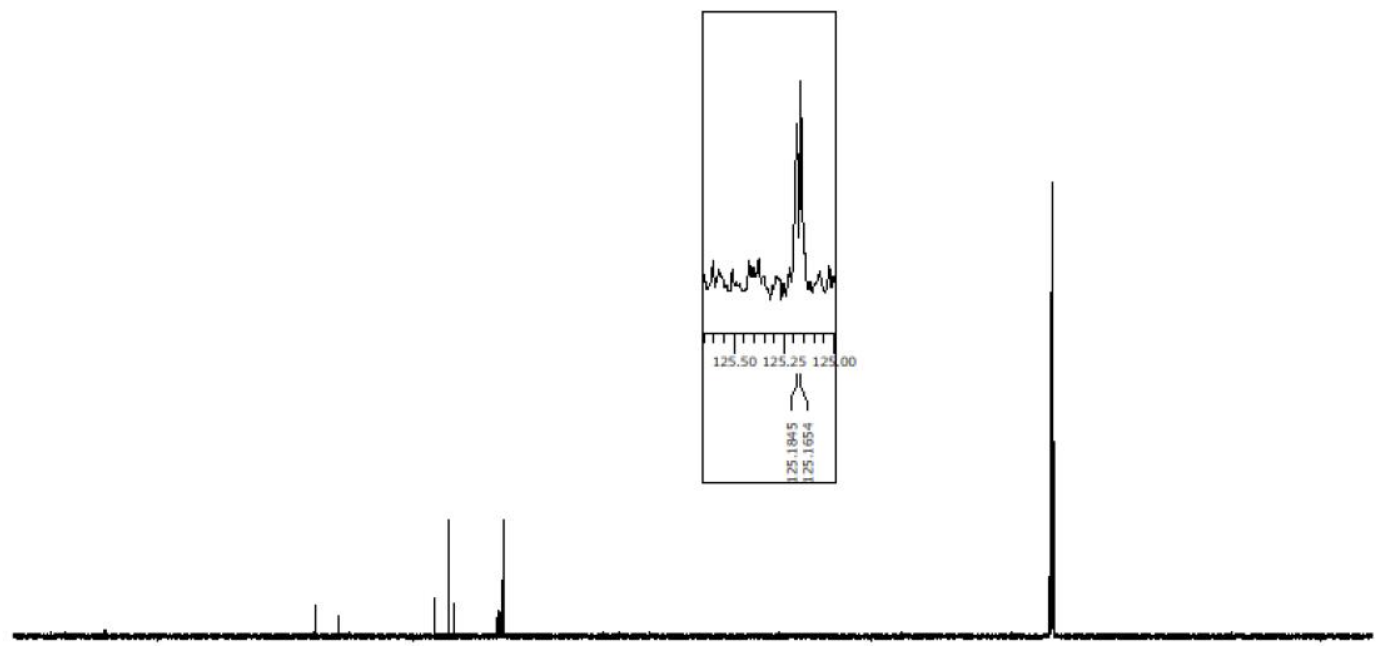

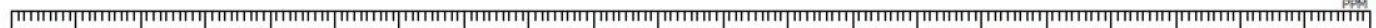

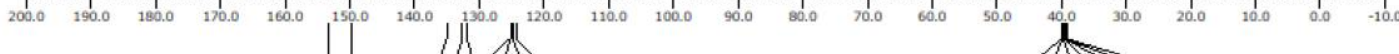
1 川

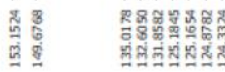

ओกा

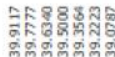


${ }^{11} \mathrm{~B}$ NMR spectra of $\mathbf{1 4 h}\left(\mathrm{DMSO}-d_{6}, 193 \mathrm{MHz}\right)$

Br

14h

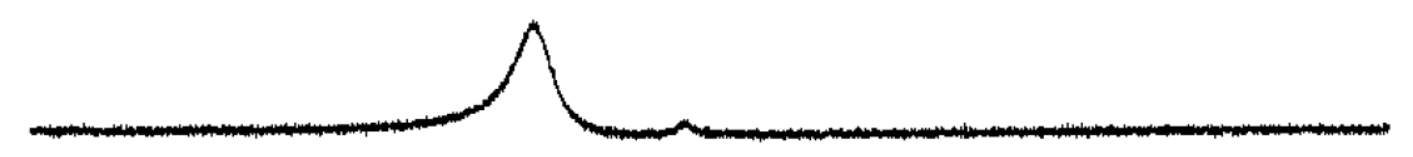

Г

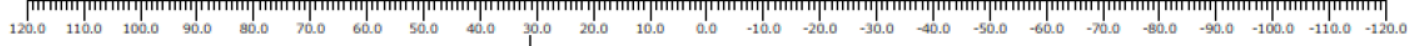
류

${ }^{1} \mathrm{H}$ NMR spectra of $\mathbf{1 5 g}\left(\mathrm{CDCl}_{3}, 400 \mathrm{MHz}\right)$

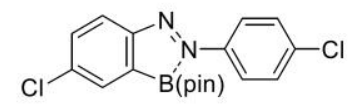

$15 \mathrm{~g}$

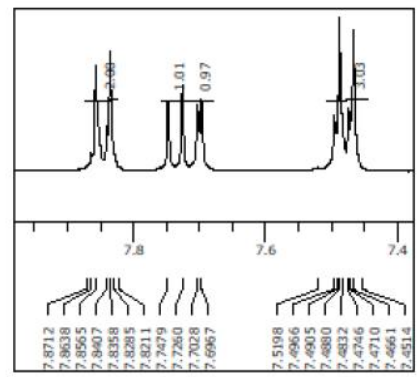

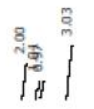

Lلl

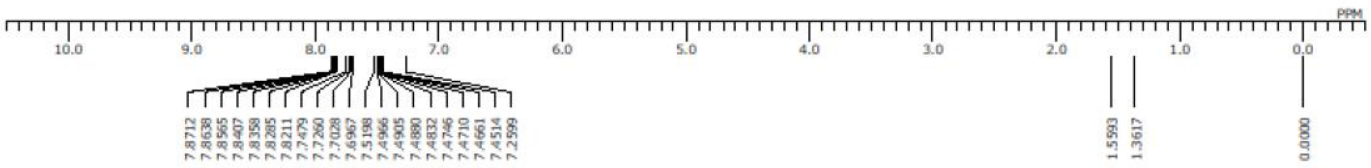


${ }^{13} \mathrm{C}$ NMR spectra of $\mathbf{1 5} \mathbf{g}\left(\mathrm{CDCl}_{3}, 100 \mathrm{MHz}\right)$<smiles></smiles>

$15 \mathrm{~g}$

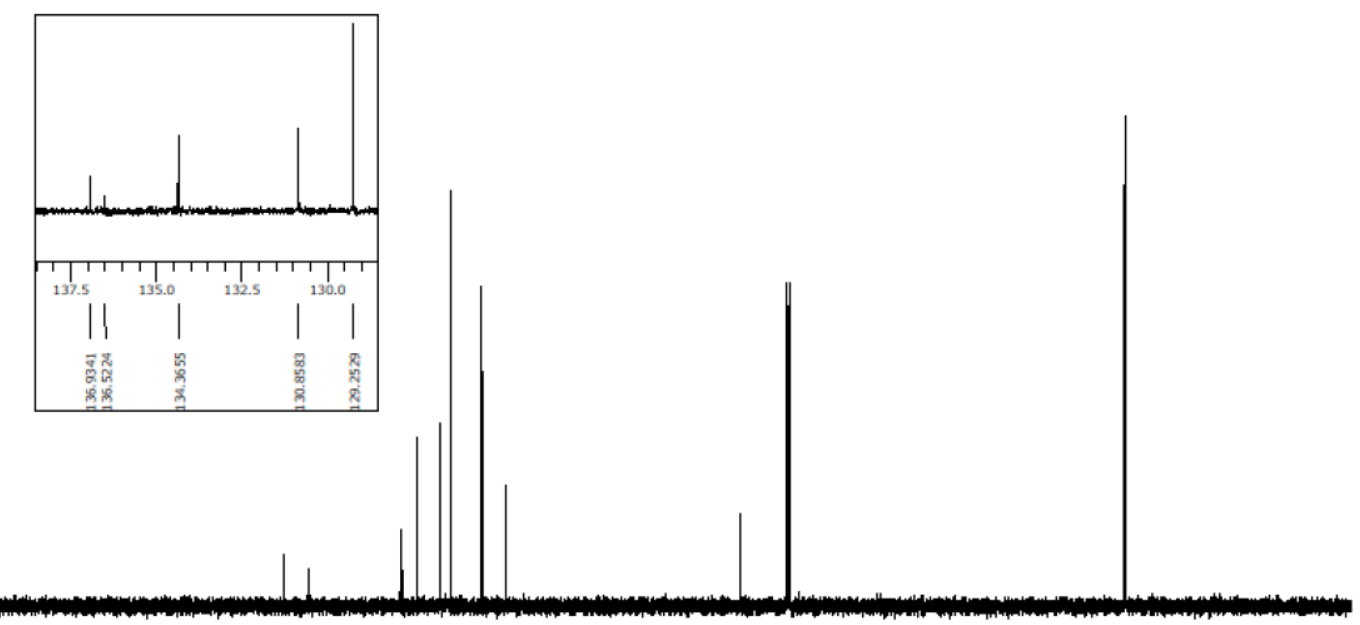

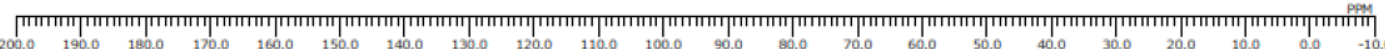

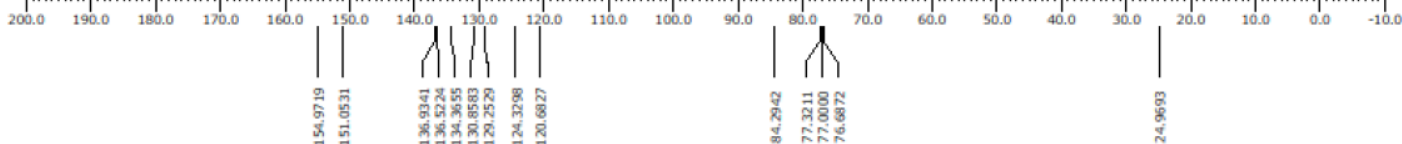

${ }^{11} \mathrm{~B}$ NMR spectra of $\mathbf{1 5} \mathbf{g}\left(\mathrm{CDCl}_{3}, 193 \mathrm{MHz}\right)$<smiles></smiles>

$15 \mathrm{~g}$

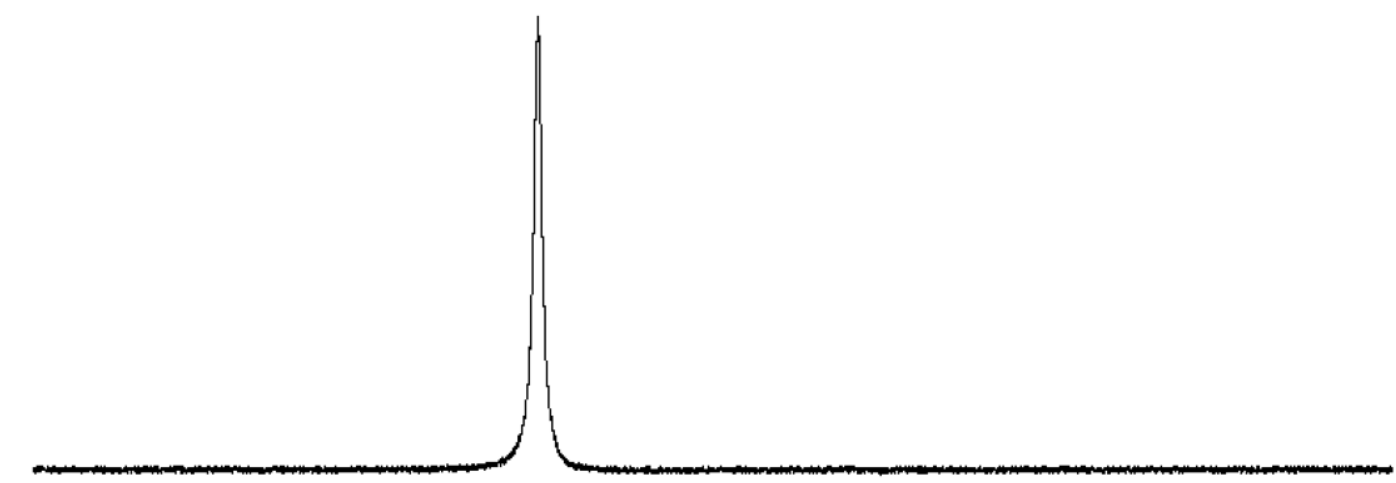

( $\begin{array}{lllllllllllllllllllllllll}{ }_{120.0} & 110.0 & 100.0 & 90.0 & 90.0 & 70.0 & 60.0 & 50.0 & 40.0 & 30.0 & 20.0 & 10.0 & 0.0 & -10.0 & -20.0 & -30.0 & -40.0 & -50.0 & -60.0 & -70.0 & -80.0 & -90.0 & -100.0 & -110.0 & -120.0\end{array}$ 勇 
${ }^{1} \mathrm{H}$ NMR spectra of $\mathbf{1 5 h}\left(\mathrm{CDCl}_{3}, 400 \mathrm{MHz}\right)$

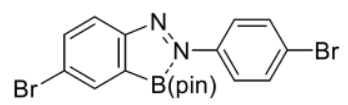

$15 \mathrm{~h}$

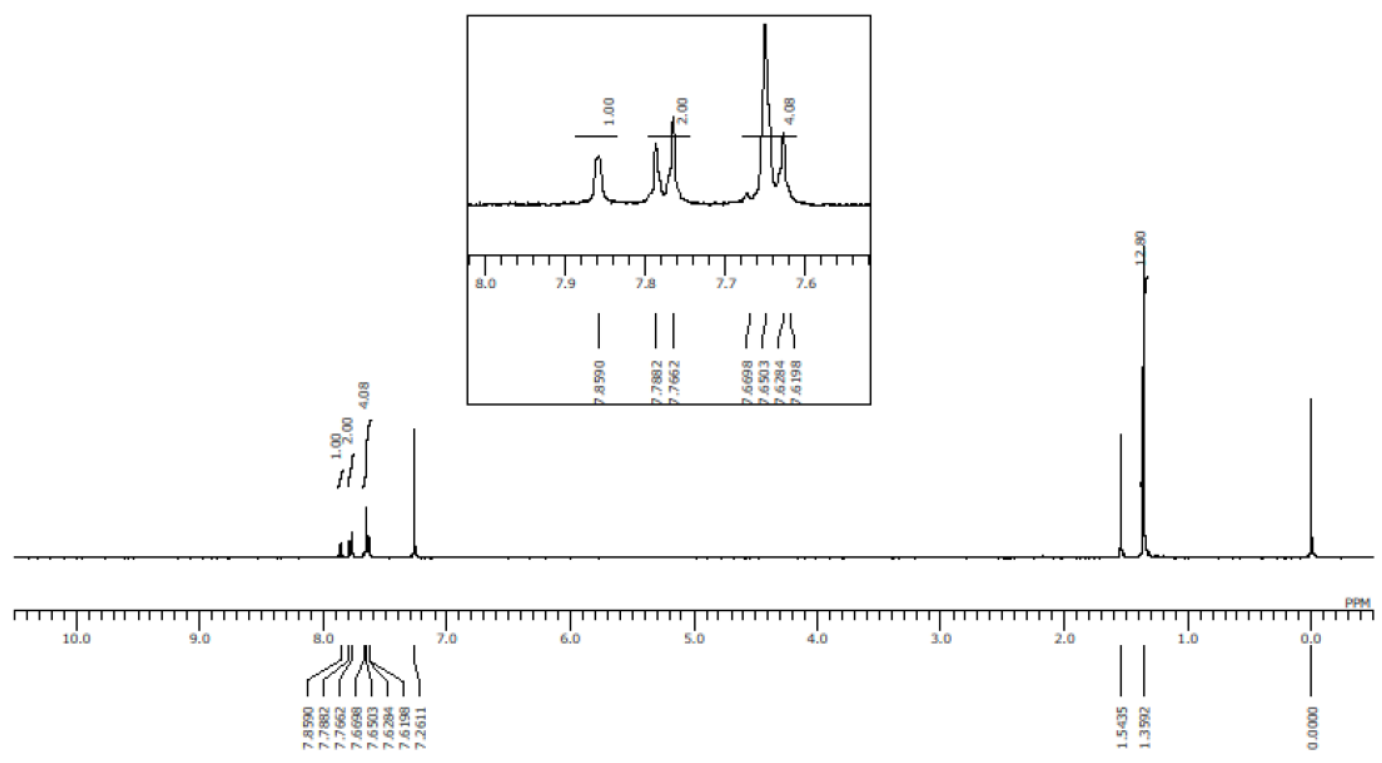

${ }^{13} \mathrm{C}$ NMR spectra of $\mathbf{1 5 h}\left(\mathrm{CDCl}_{3}, 100 \mathrm{MHz}\right)$

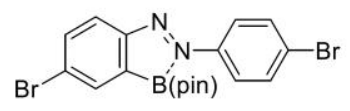

15h

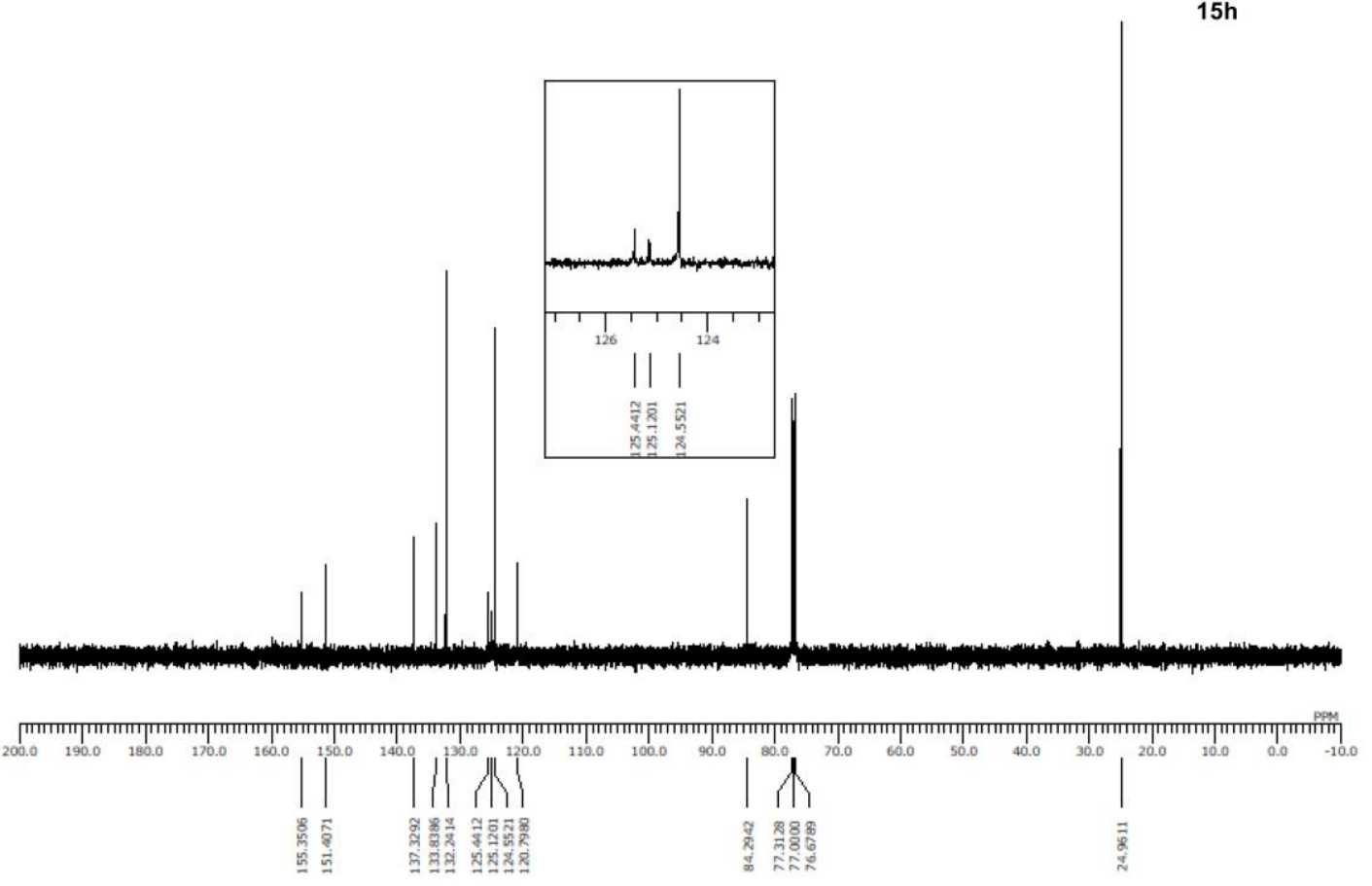


${ }^{11} \mathrm{~B}$ NMR spectra of $\mathbf{1 5 h}\left(\mathrm{CDCl}_{3}, 193 \mathrm{MHz}\right)$

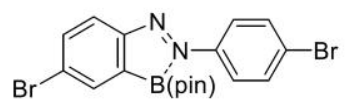

$15 \mathrm{~h}$

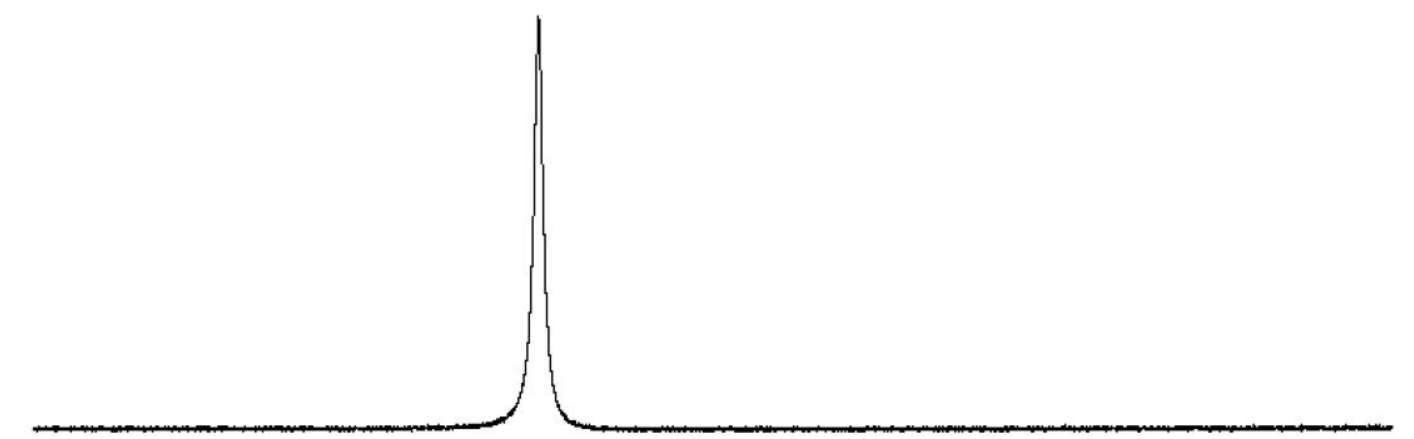

[ $\begin{array}{llllllllllllllllllllllllll}{ }_{120.0} & 110.0 & 100.0 & 90.0 & 90.0 & 70.0 & 60.0 & 50.0 & 40.0 & 30.0 & 20.0 & 10.0 & 0.0 & -10.0 & -20.0 & -30.0 & -40.0 & -50.0 & -60.0 & -70.0 & -80.0 & -90.0 & -100.0 & -110.0 & -120.0\end{array}$

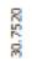

${ }^{1} \mathrm{H}$ NMR spectra of $\mathbf{1 6 a}\left(\mathrm{CDCl}_{3}, 400 \mathrm{MHz}\right)$
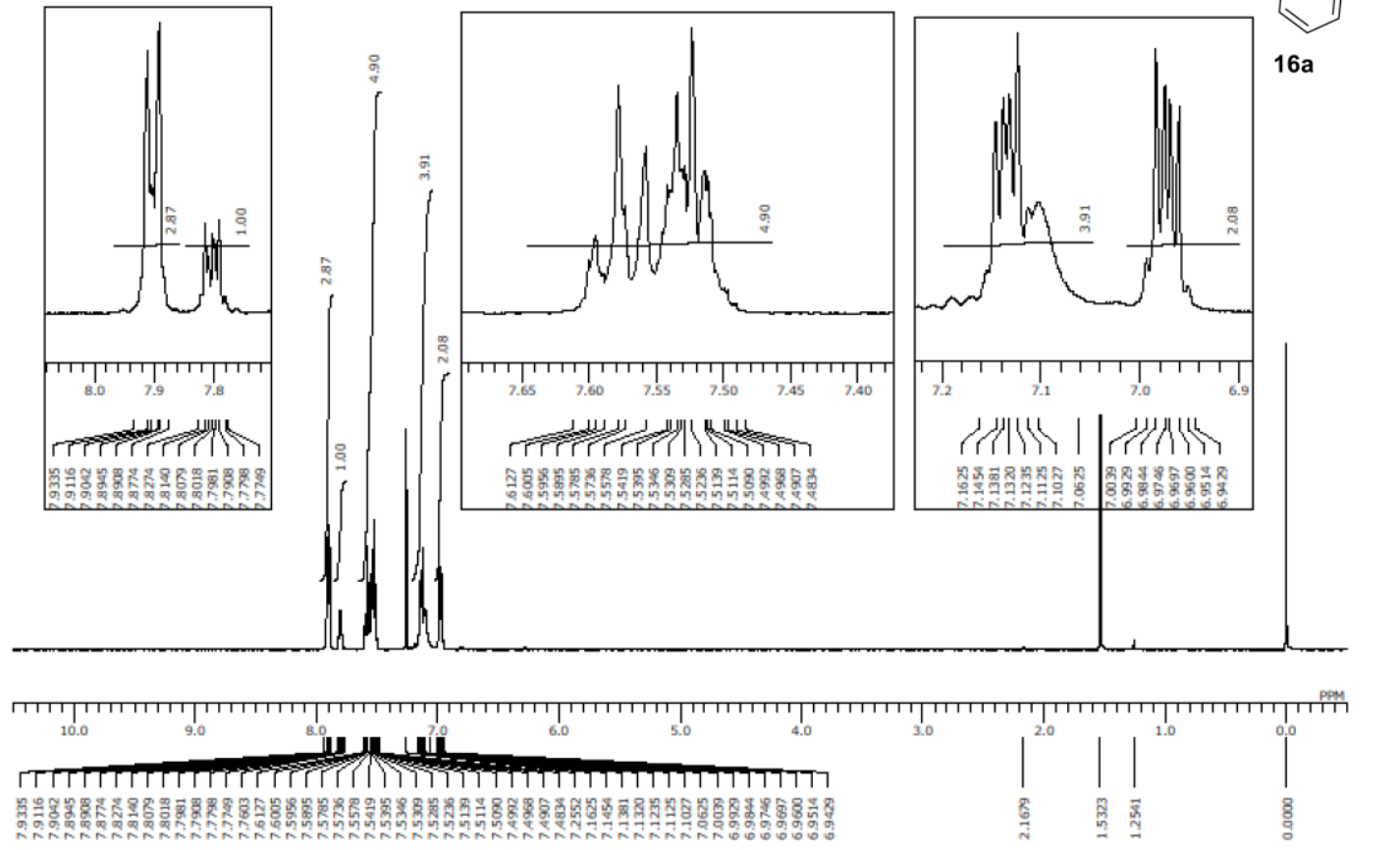
${ }^{13} \mathrm{C}$ NMR spectra of $\mathbf{1 6 a}\left(\mathrm{CDCl}_{3}, 150 \mathrm{MHz}\right)$
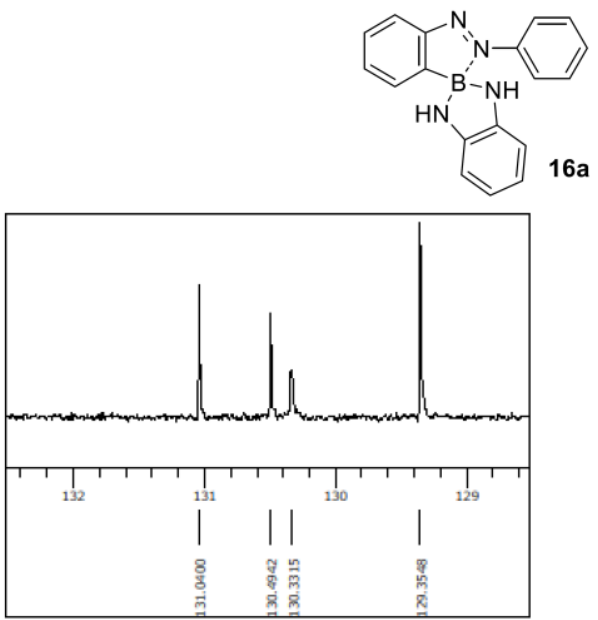

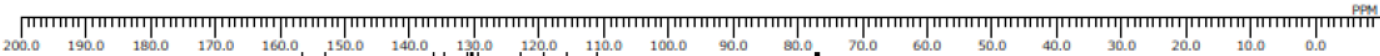
$\left.\right|_{0} ^{150.0}$

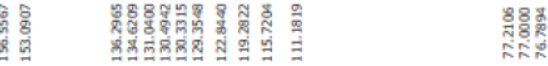

${ }^{11} \mathrm{~B}$ NMR spectra of $\mathbf{1 6 a}\left(\mathrm{CDCl}_{3}, 193 \mathrm{MHz}\right)$

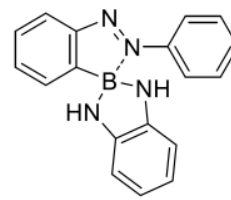

$16 a$

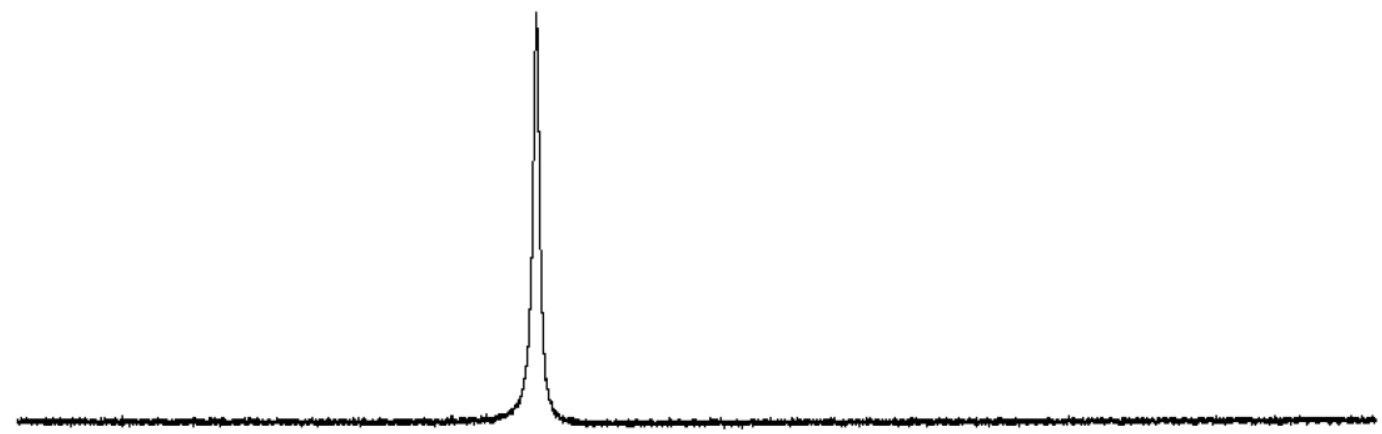

हा"

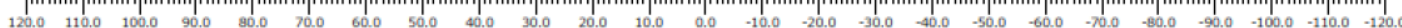
离 
${ }^{1} \mathrm{H}$ NMR spectra of $\mathbf{1 7} \mathbf{a}\left(\mathrm{CD}_{2} \mathrm{Cl}_{2}, 600 \mathrm{MHz}\right)$

${ }_{B}^{\prime \prime}\left(\mathrm{C}_{6} \mathrm{~F}_{5}\right)_{2}$

$17 \mathrm{a}$

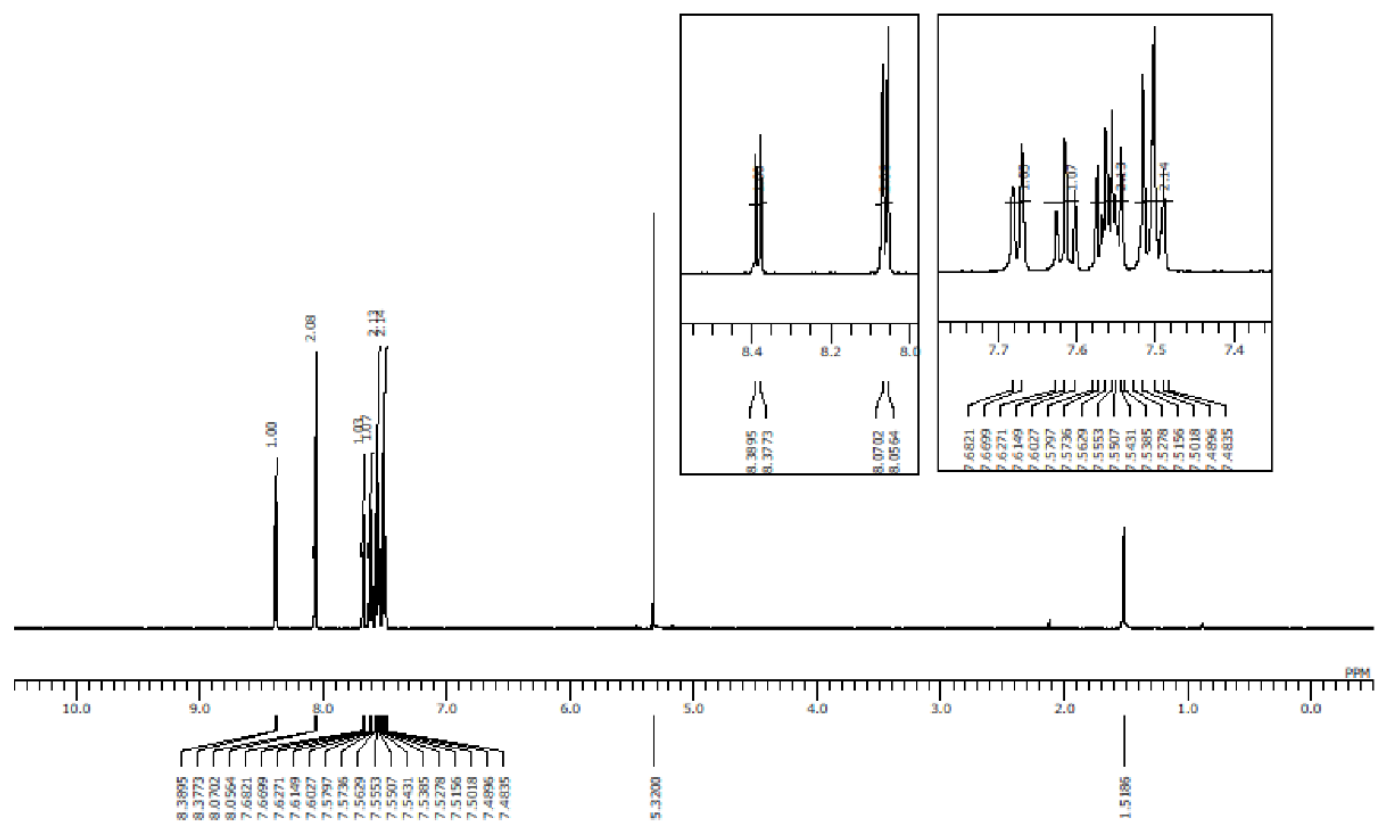

${ }^{13} \mathrm{C}$ NMR spectra of $\mathbf{1 7 a}\left(\mathrm{CD}_{2} \mathrm{Cl}_{2}, 150 \mathrm{MHz}\right)$

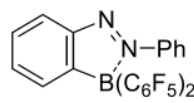

$17 \mathrm{a}$
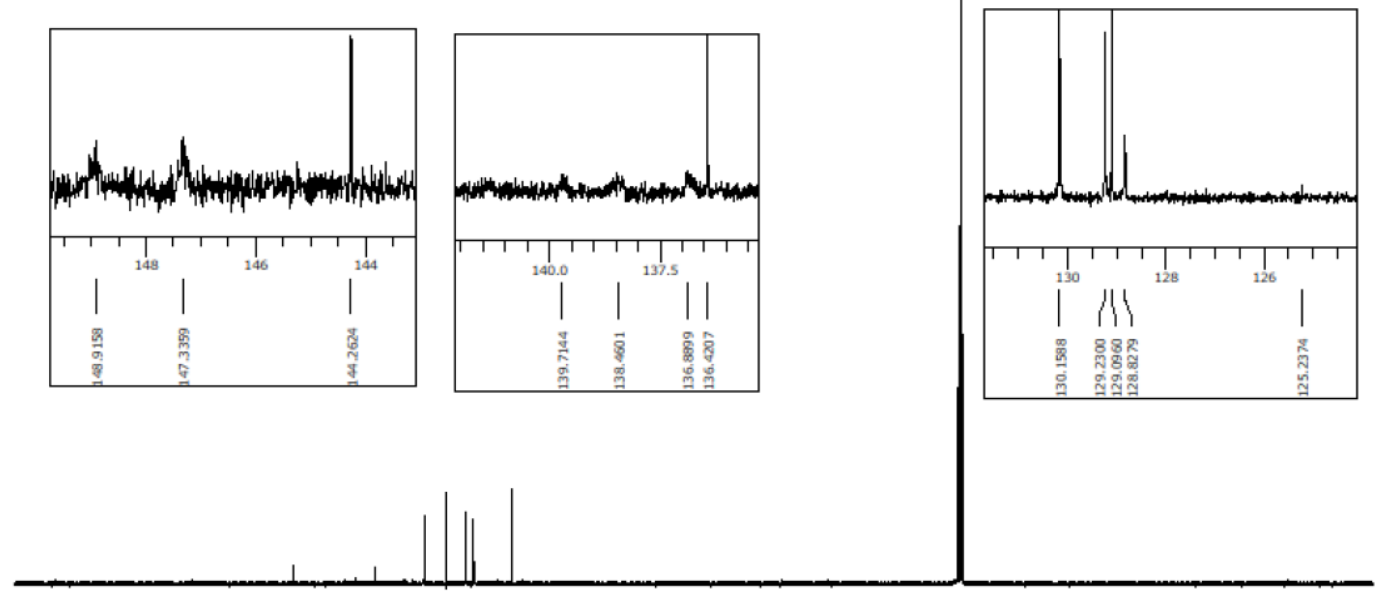

[

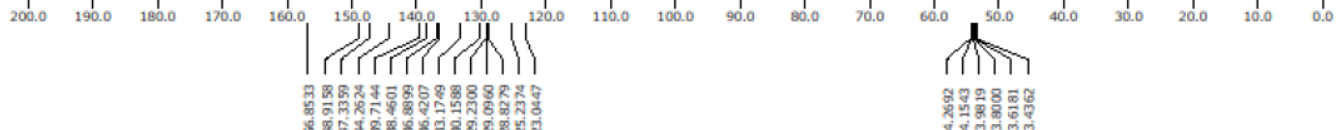


${ }^{11} \mathrm{~B}$ NMR spectra of $\mathbf{1 7} \mathbf{a}\left(\mathrm{CD}_{2} \mathrm{Cl}_{2}, 193 \mathrm{MHz}\right)$

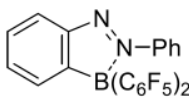

$17 a$

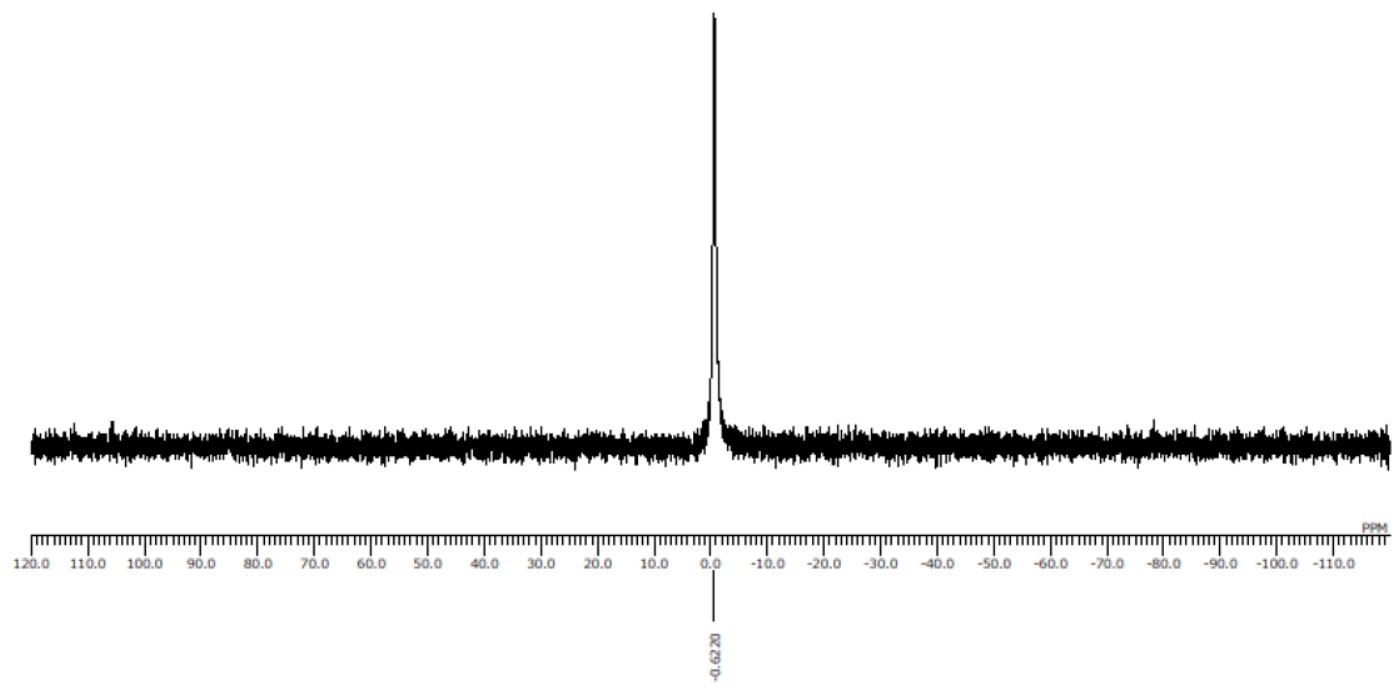

${ }^{1} \mathrm{H}$ NMR spectra of $4 \mathbf{a a}\left(\mathrm{CD}_{2} \mathrm{Cl}_{2}, 600 \mathrm{MHz}\right)$
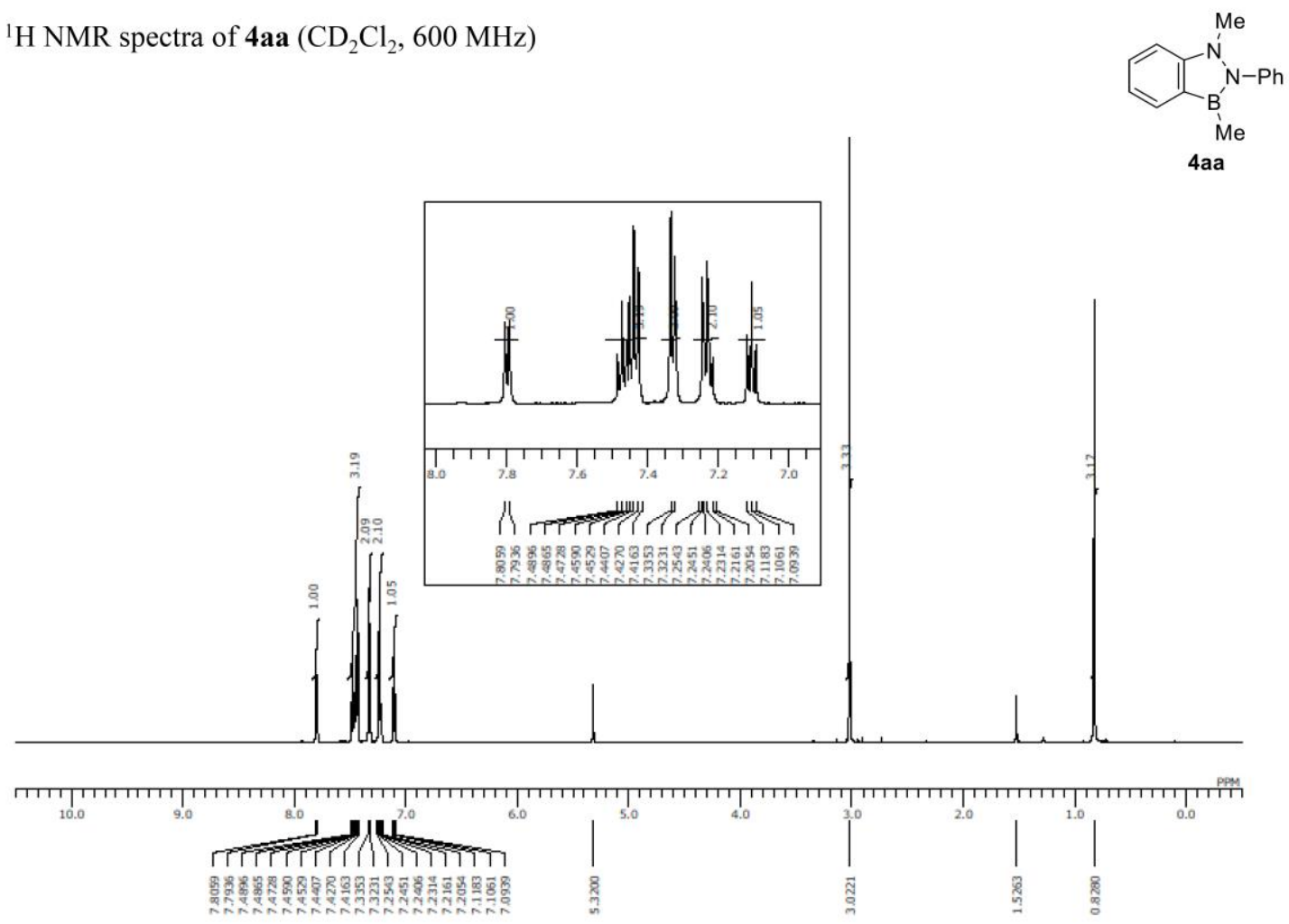
${ }^{13} \mathrm{C}$ NMR spectra of $4 \mathbf{a a}\left(\mathrm{CD}_{2} \mathrm{Cl}_{2}, 150 \mathrm{MHz}\right)$
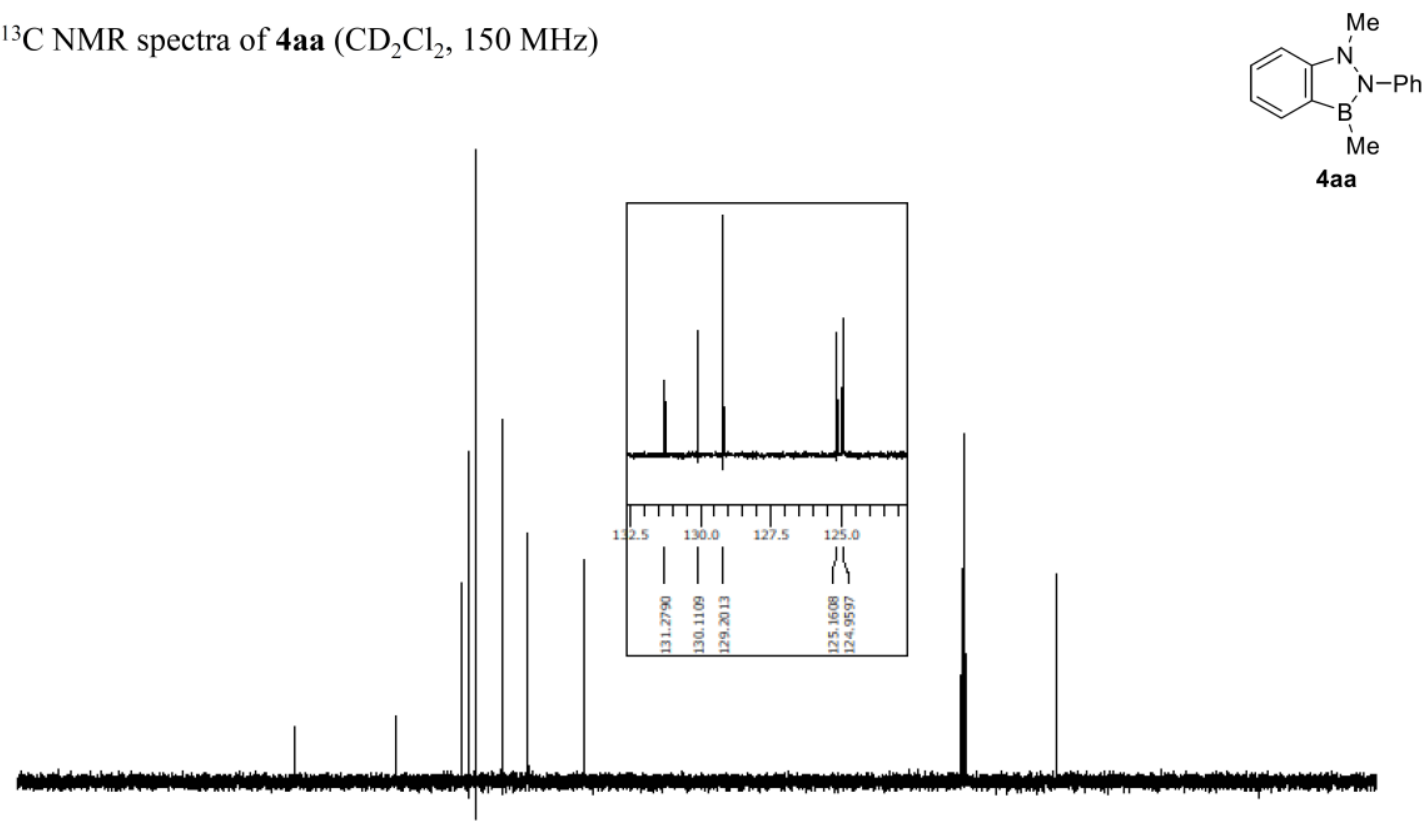

[

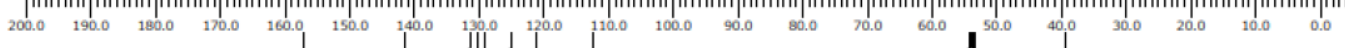

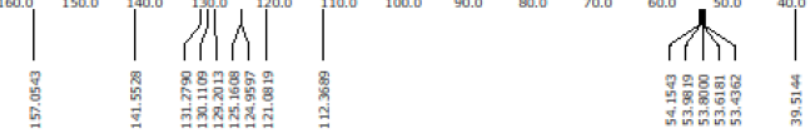

${ }^{11} \mathrm{~B}$ NMR spectra of $\mathbf{4 a a}\left(\mathrm{CD}_{2} \mathrm{Cl}_{2}, 193 \mathrm{MHz}\right)$

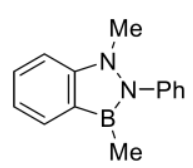

4aa

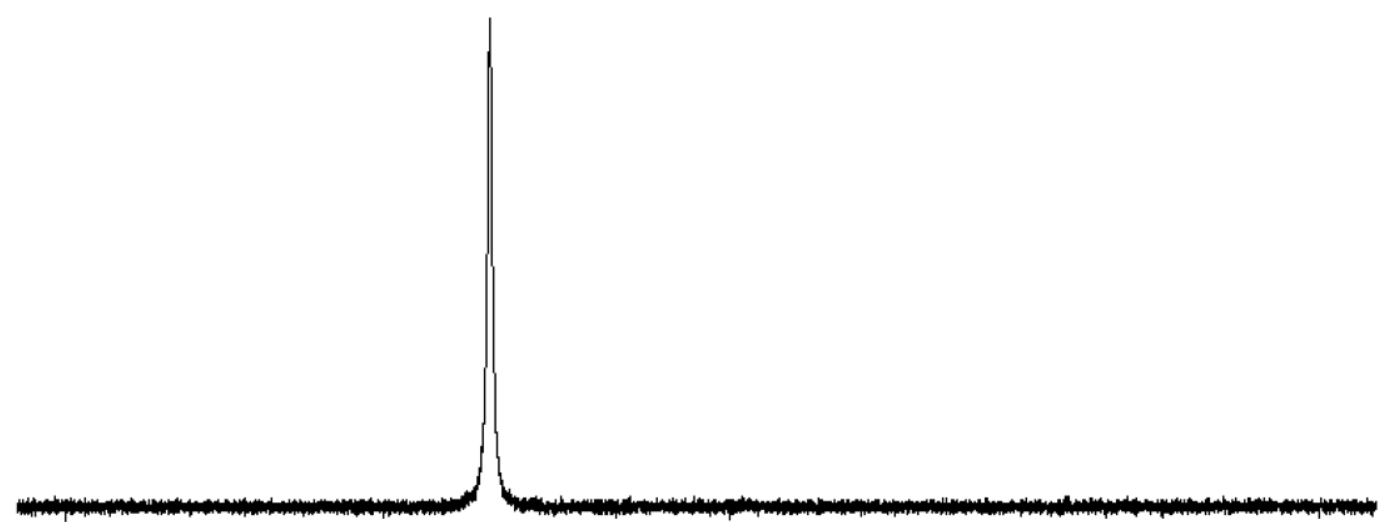

[

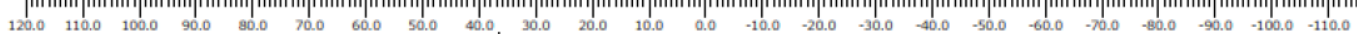


${ }^{1} \mathrm{H}$ NMR spectra of $\mathbf{4 a b}\left(\mathrm{C}_{6} \mathrm{D}_{6}, 600 \mathrm{MHz}\right)$
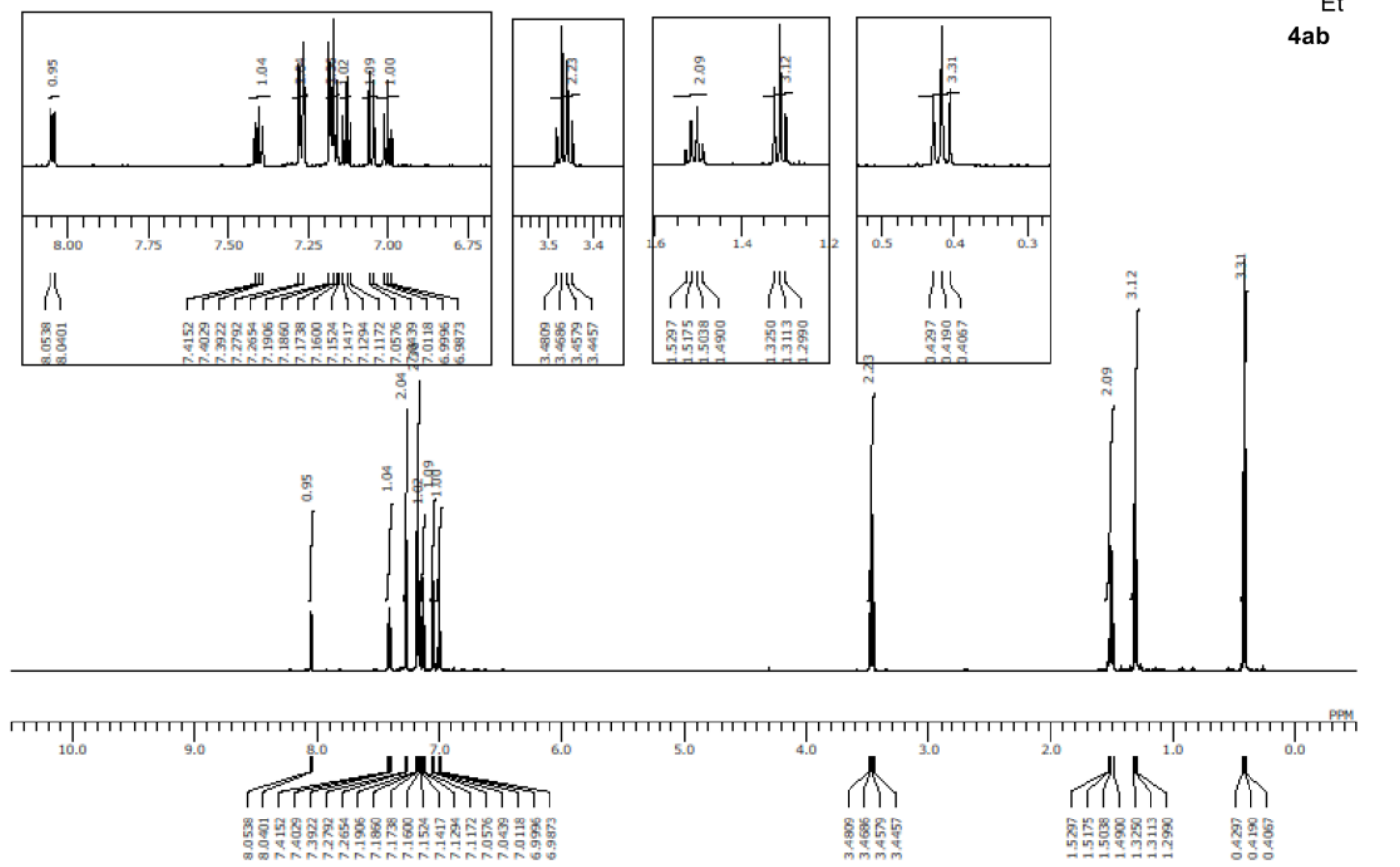

${ }^{13} \mathrm{C}$ NMR spectra of $\mathbf{4 a b}\left(\mathrm{C}_{6} \mathrm{D}_{6}, 150 \mathrm{MHz}\right)$

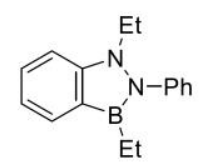

$4 \mathrm{ab}$

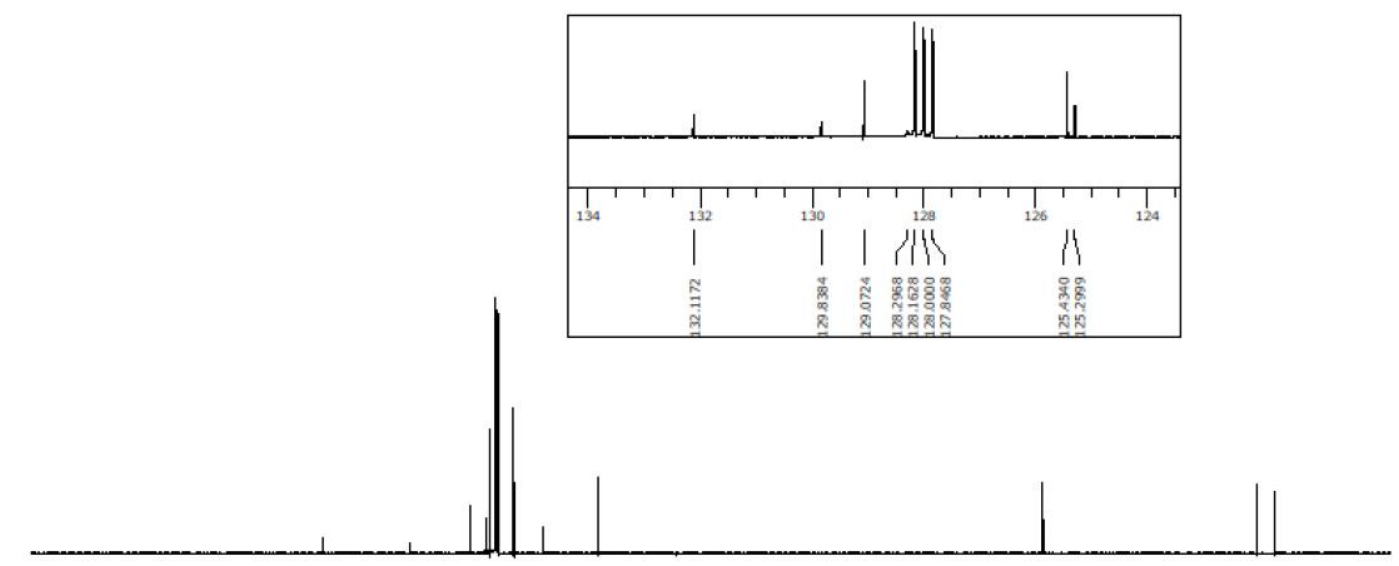

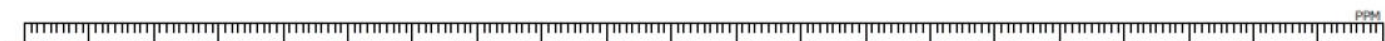

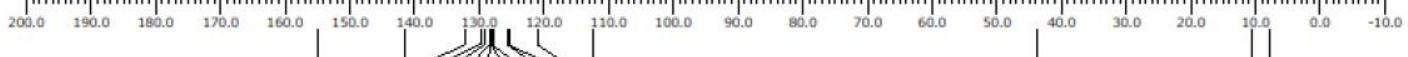

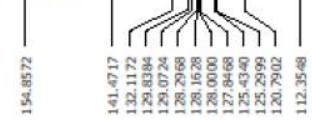


${ }^{11} \mathrm{~B}$ NMR spectra of $\mathbf{4 a b}\left(\mathrm{C}_{6} \mathrm{D}_{6}, 193 \mathrm{MHz}\right)$
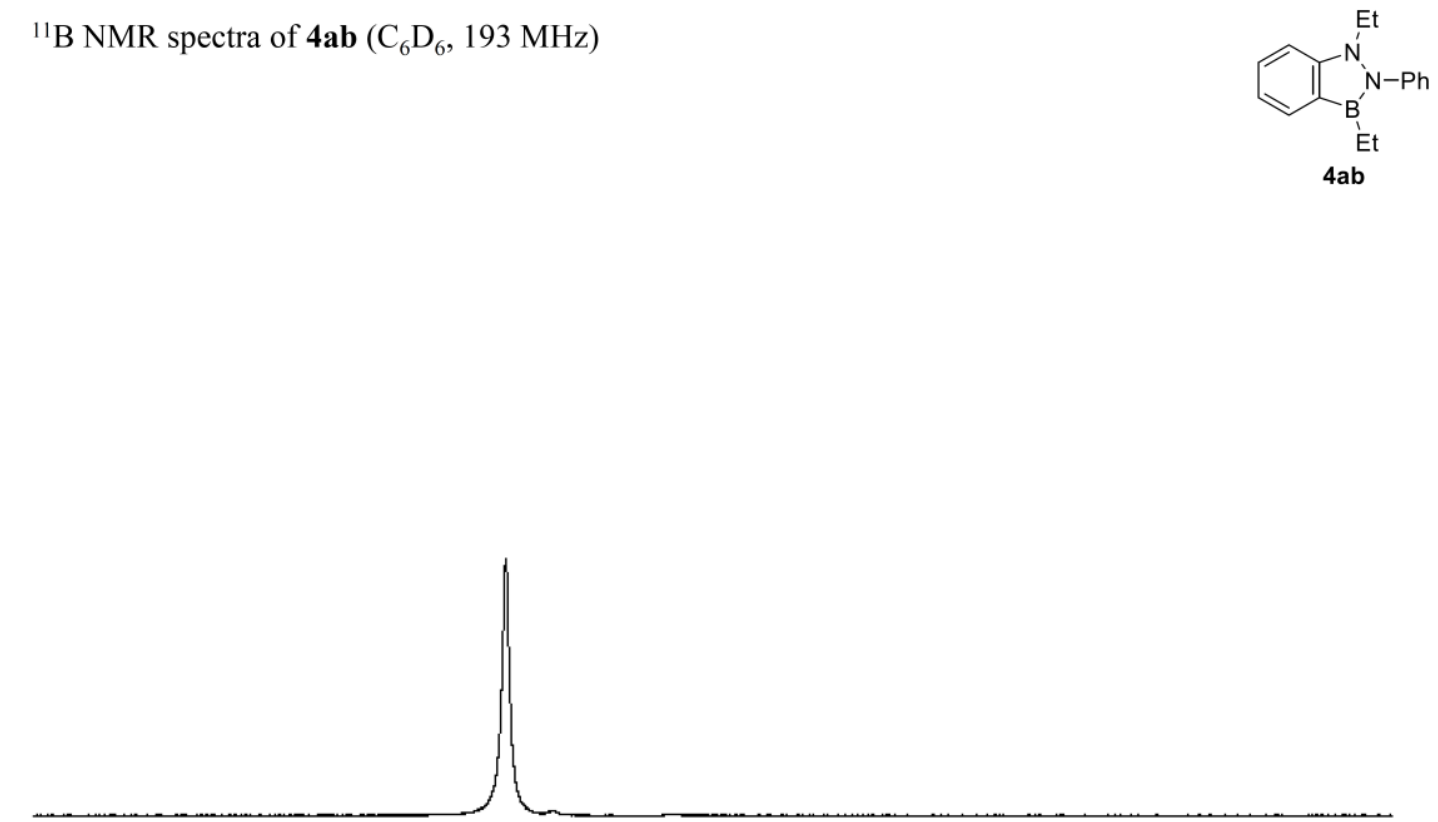

Г $\begin{array}{lllllllllllllllllllllllllll}120.0 & 110.0 & 100.0 & 90.0 & 80.0 & 70.0 & 60.0 & 50.0 & 40.0 & 30.0 & 20.0 & 10.0 & 0.0 & -10.0 & -20.0 & -30.0 & -40.0 & -50.0 & -60.0 & -70.0 & -80.0 & -90.0 & -100.0 & -110.0 & \end{array}$

${ }^{1} \mathrm{H}$ NMR spectra of $\mathbf{4 a c}\left(\mathrm{CDCl}_{3}, 400 \mathrm{MHz}\right)$
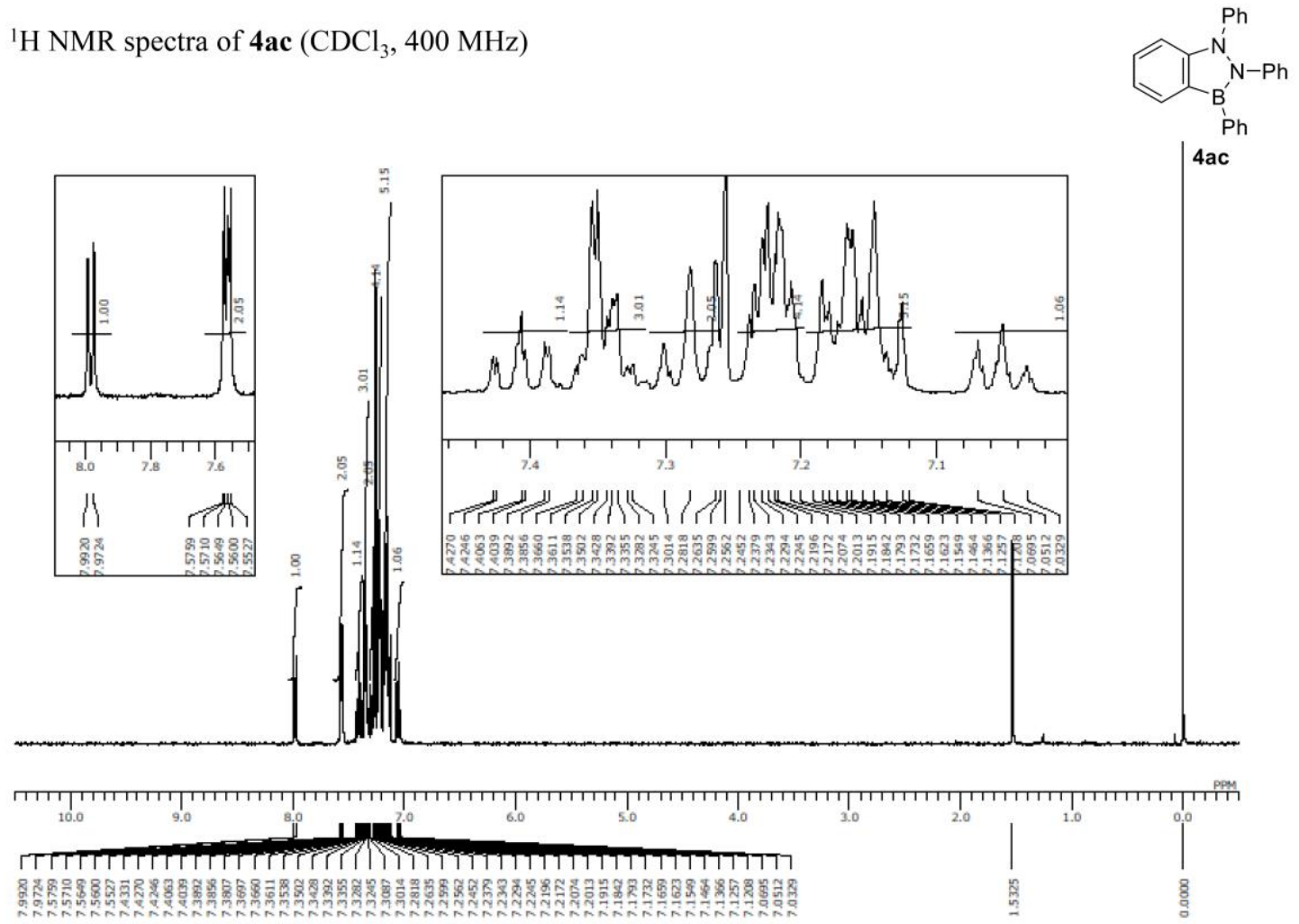
${ }^{13} \mathrm{C}$ NMR spectra of $\mathbf{4 a c}\left(\mathrm{CDCl}_{3}, 100 \mathrm{MHz}\right)$
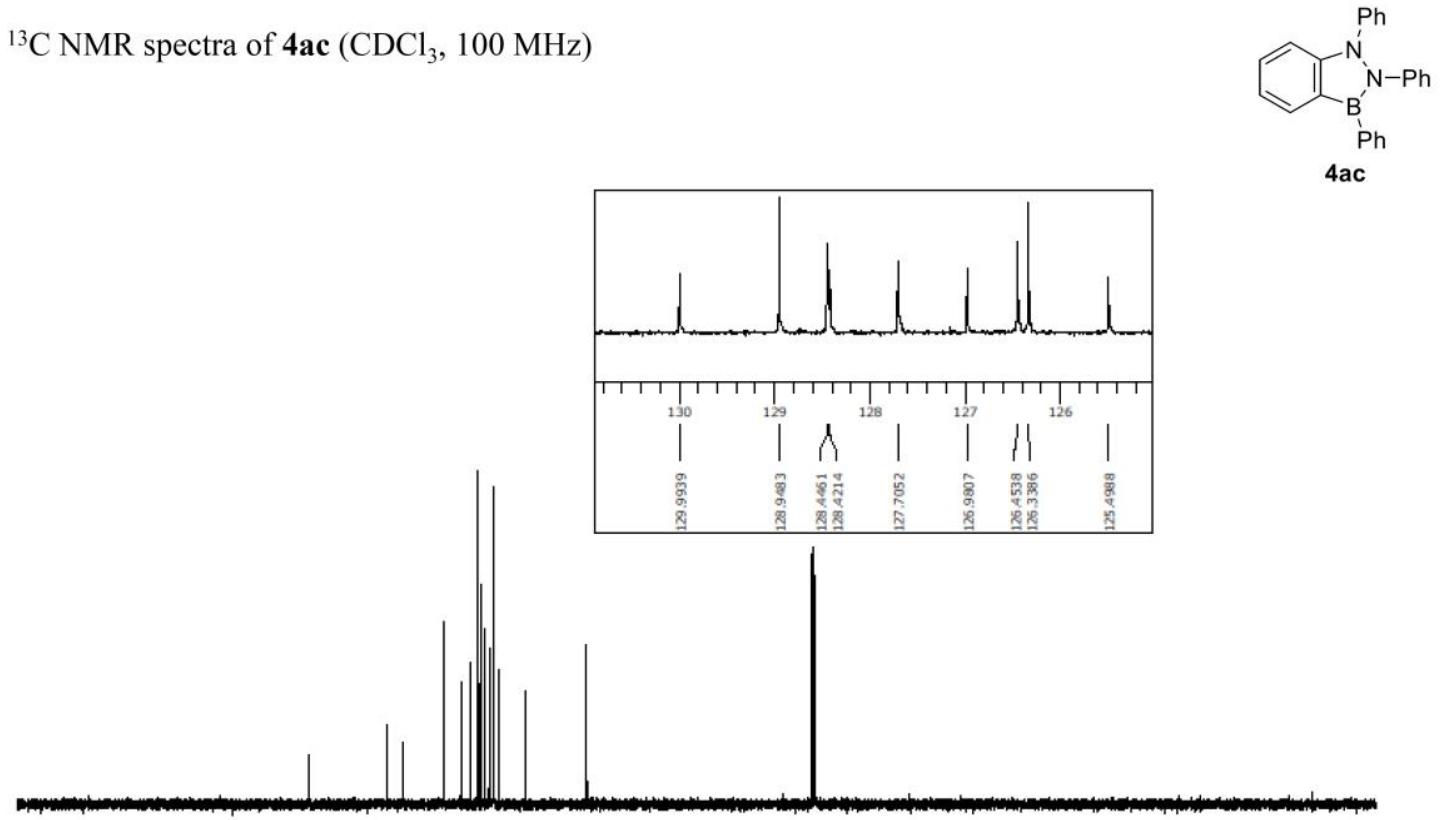

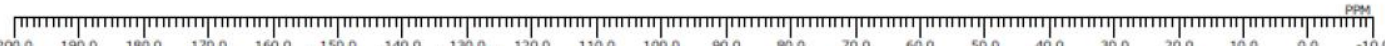

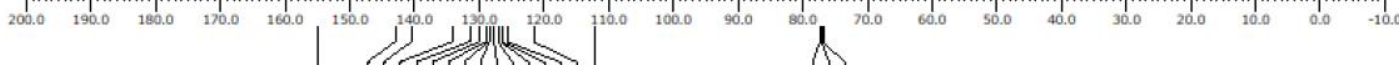
तिराफा

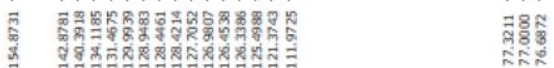

${ }^{11} \mathrm{~B}$ NMR spectra of $\mathbf{4 a c}\left(\mathrm{CDCl}_{3}, 193 \mathrm{MHz}\right)$
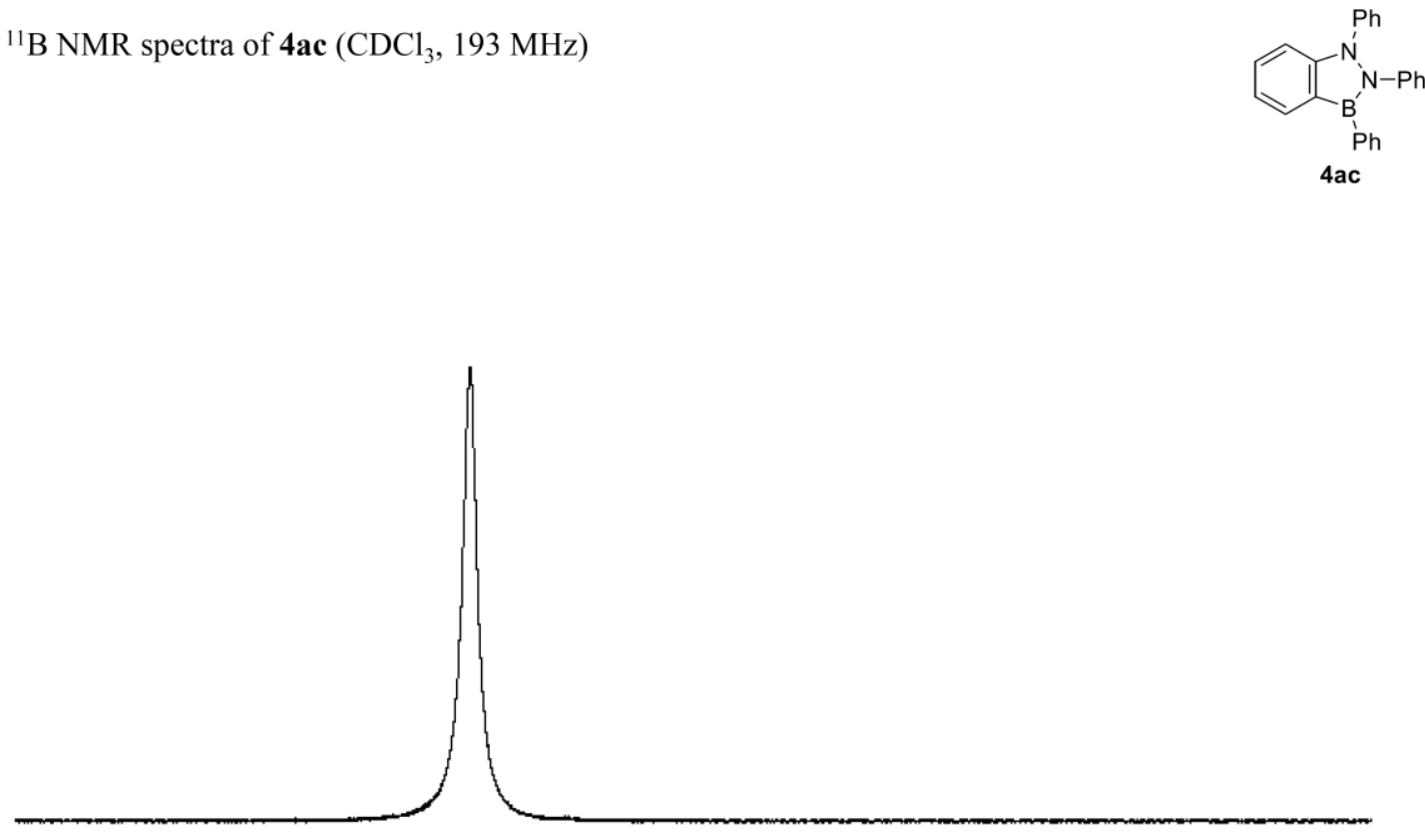

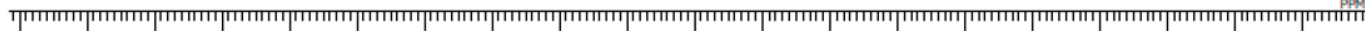

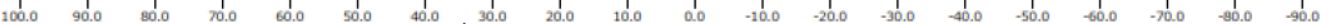


${ }^{1} \mathrm{H}$ NMR spectra of $\mathbf{4 a d}\left(\mathrm{C}_{6} \mathrm{D}_{6}, 400 \mathrm{MHz}\right)$
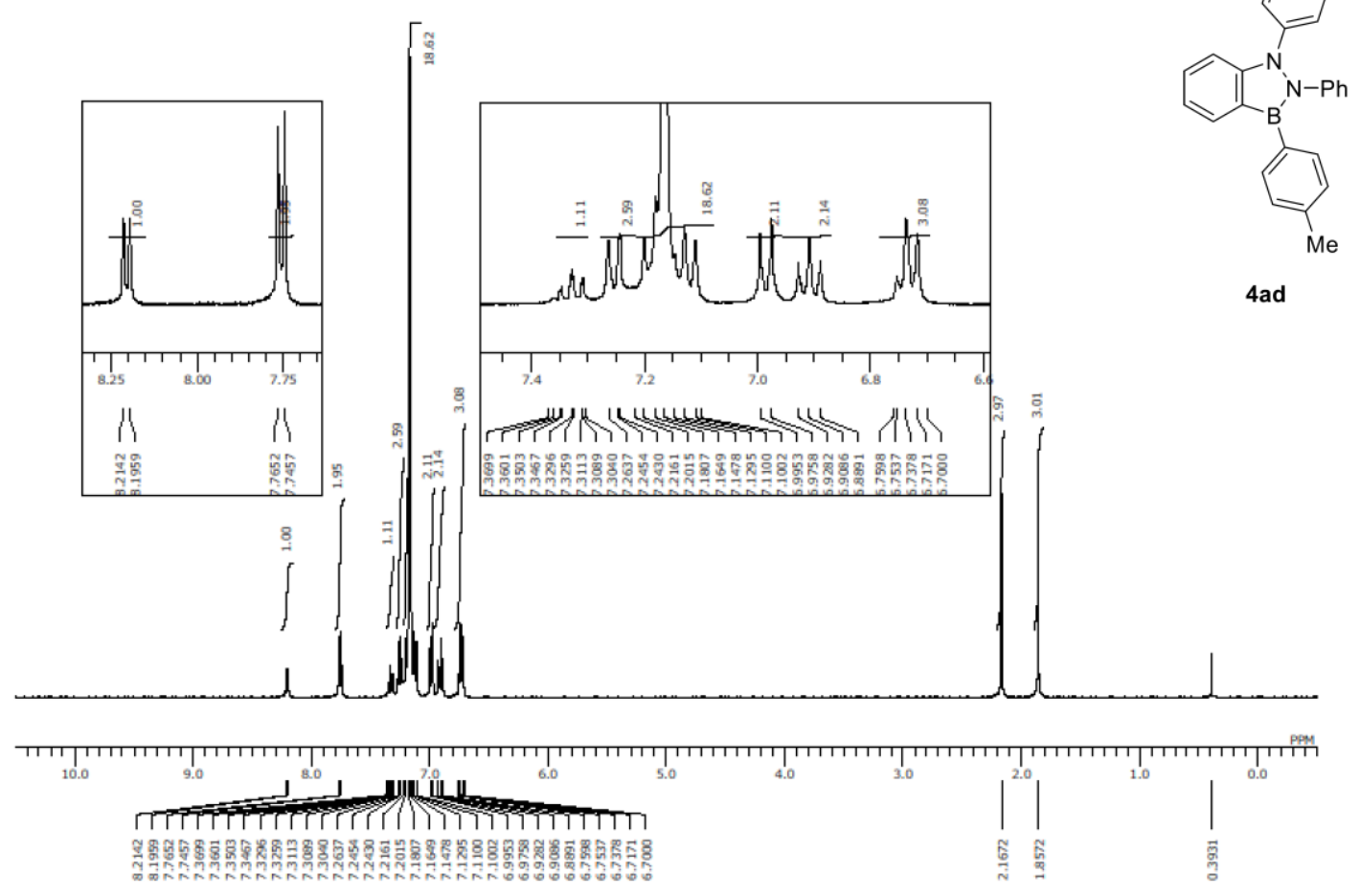

${ }^{13} \mathrm{C}$ NMR spectra of $\mathbf{4 a d}\left(\mathrm{C}_{6} \mathrm{D}_{6}, 150 \mathrm{MHz}\right)$
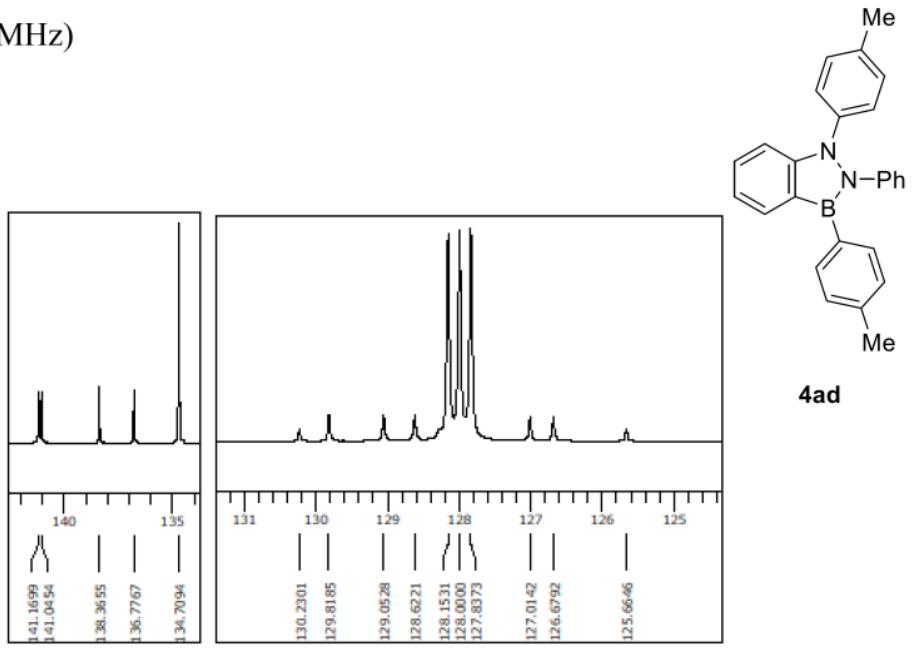

(

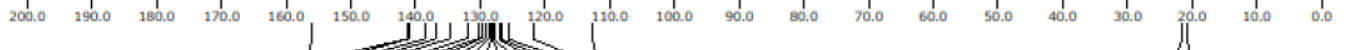
rip

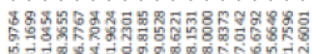


${ }^{11} \mathrm{~B}$ NMR spectra of $\mathbf{4 a b}\left(\mathrm{C}_{6} \mathrm{D}_{6}, 193 \mathrm{MHz}\right)$

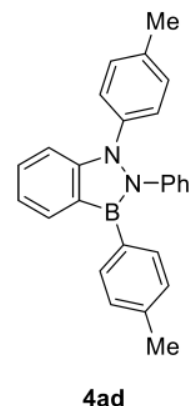

[ $\begin{array}{lllllllllllllllllllllllll}120.0 & 110.0 & 100.0 & 90.0 & 80.0 & 70.0 & 60.0 & 50.0 & 40.0 & 30.0 & 20.0 & 10.0 & 0.0 & -10.0 & -20.0 & -30.0 & -40.0 & -50.0 & -60.0 & -70.0 & -80.0 & -90.0 & -100.0 & -110.0 & -120.0\end{array}$

${ }^{1} \mathrm{H}$ NMR spectra of $\mathbf{4 a e}\left(\mathrm{C}_{6} \mathrm{D}_{6}, 400 \mathrm{MHz}\right)$
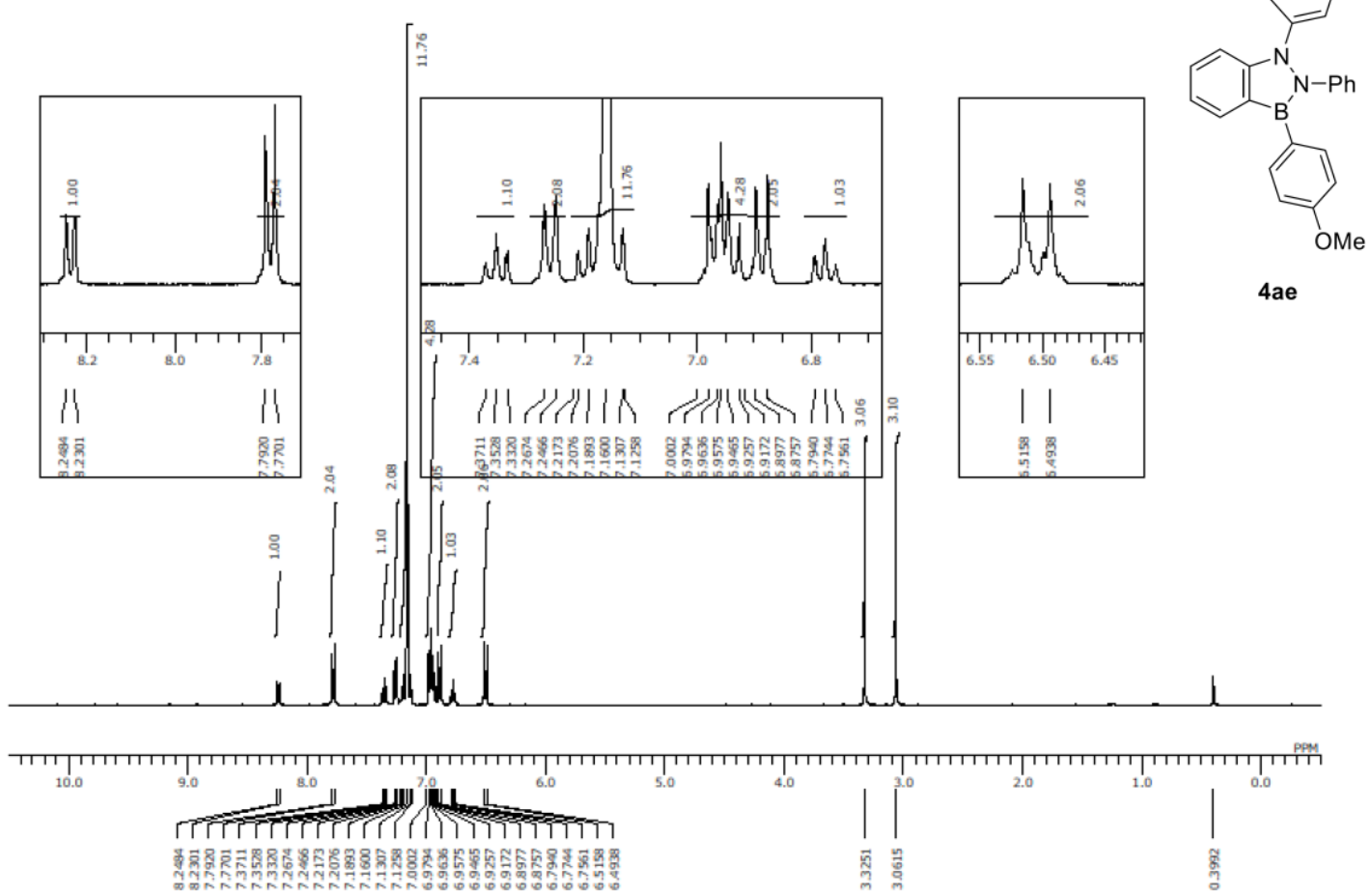
${ }^{13} \mathrm{C}$ NMR spectra of $4 \mathbf{a e}\left(\mathrm{C}_{6} \mathrm{D}_{6}, 150 \mathrm{MHz}\right)$

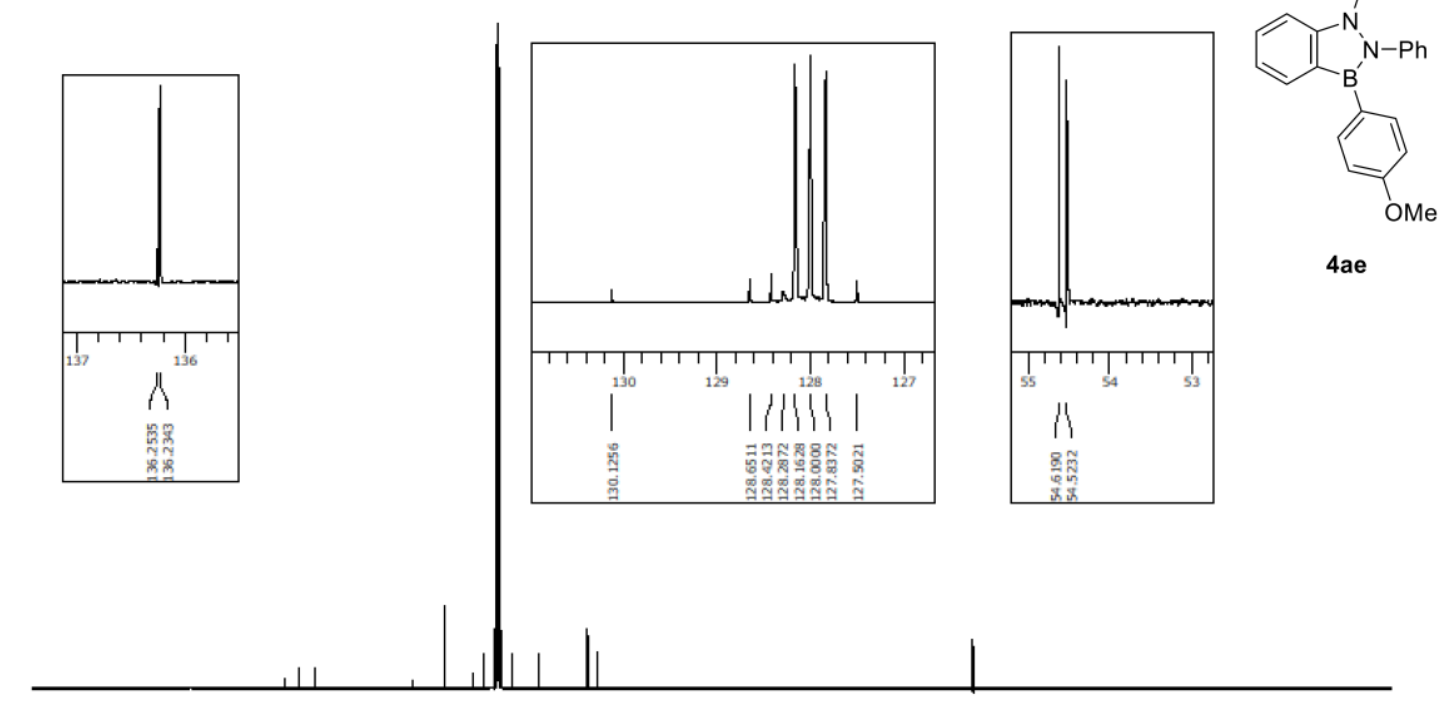

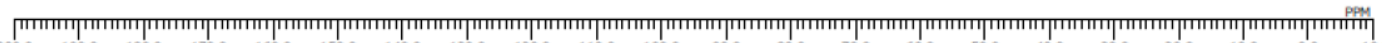
$\left.{ }_{200.0}^{200.0} 0_{180.0}\right|_{170.0} ^{160.0}$

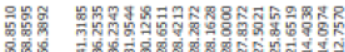

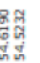

${ }^{11} \mathrm{~B}$ NMR spectra of $\mathbf{4 a e}\left(\mathrm{C}_{6} \mathrm{D}_{6}, 193 \mathrm{MHz}\right)$

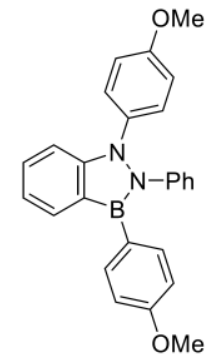

4ae

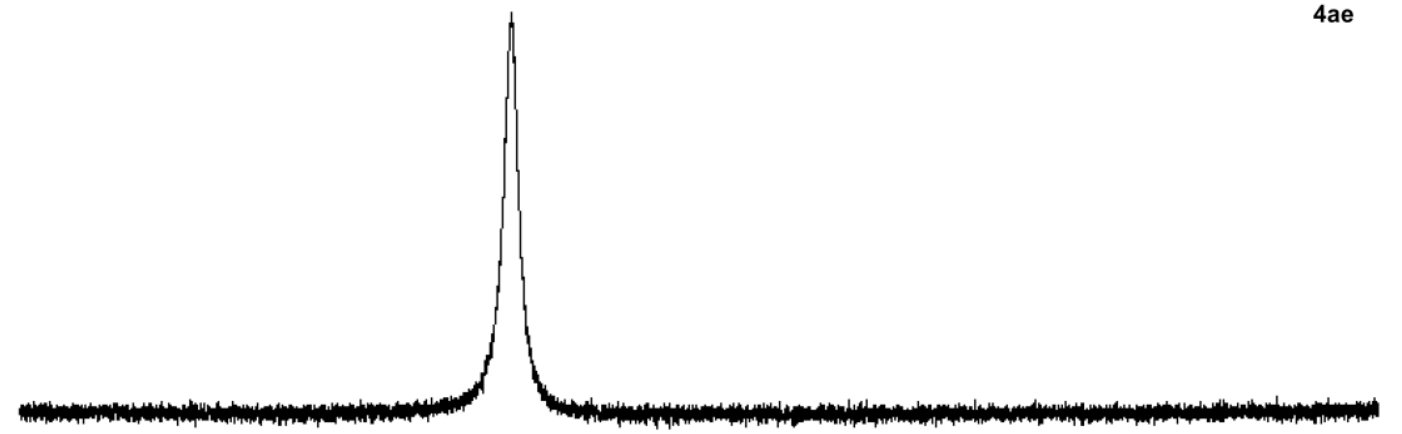

[ $\begin{array}{llllllllllllllllllllllllll}120.0 & 110.0 & 100.0 & 90.0 & 90.0 & 70.0 & 60.0 & 50.0 & 40.0 & 30.0 & 20.0 & 10.0 & 0.0 & -10.0 & -20.0 & -30.0 & -40.0 & -50.0 & -60.0 & -70.0 & -80.0 & -90.0 & -100.0 & -110.0 & -120.0\end{array}$ 
${ }^{1} \mathrm{H}$ NMR spectra of $\mathbf{4 a f}\left(\mathrm{C}_{6} \mathrm{D}_{6}, 400 \mathrm{MHz}\right)$
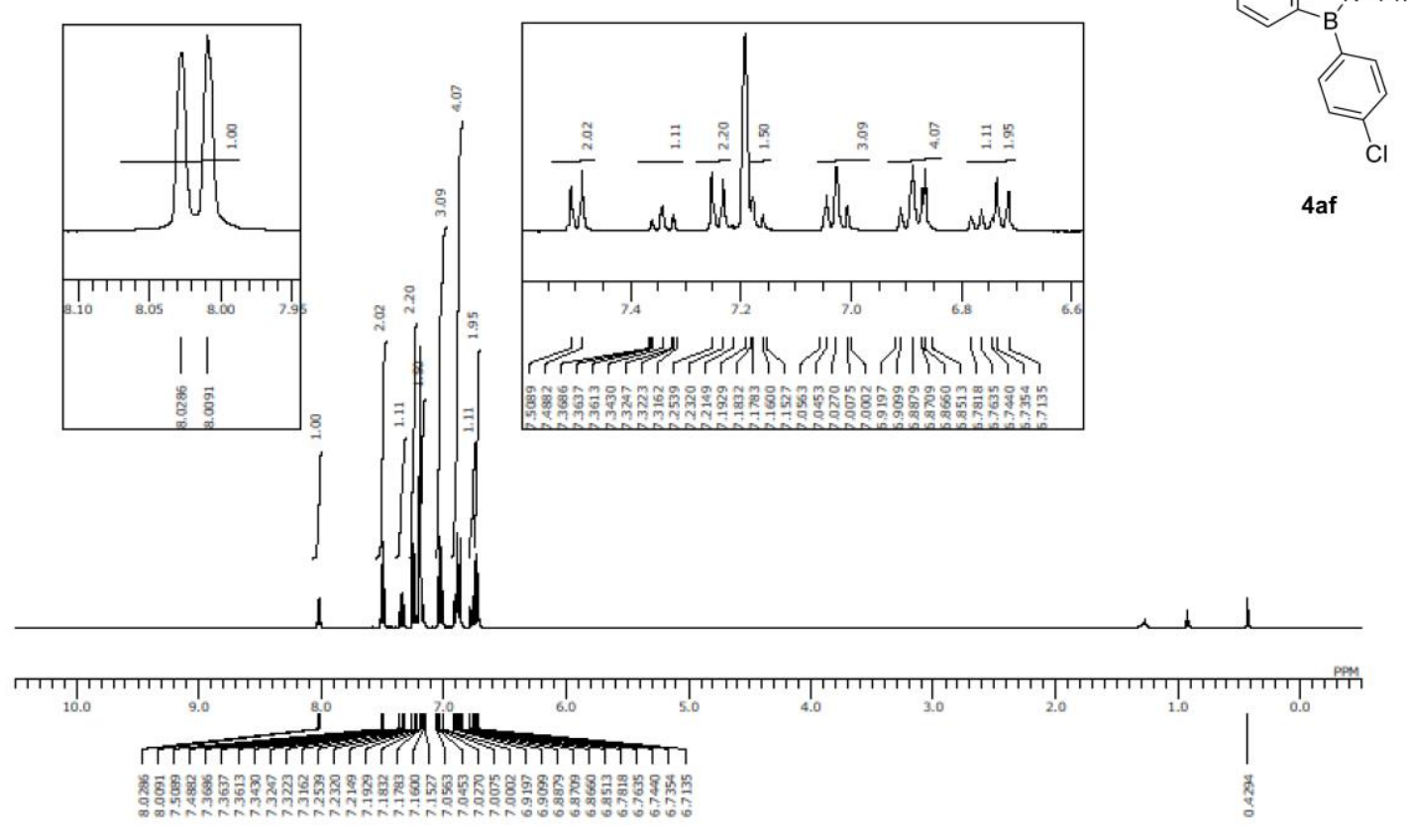

${ }^{13} \mathrm{C}$ NMR spectra of $\mathbf{4 a f}\left(\mathrm{C}_{6} \mathrm{D}_{6}, 150 \mathrm{MHz}\right)$
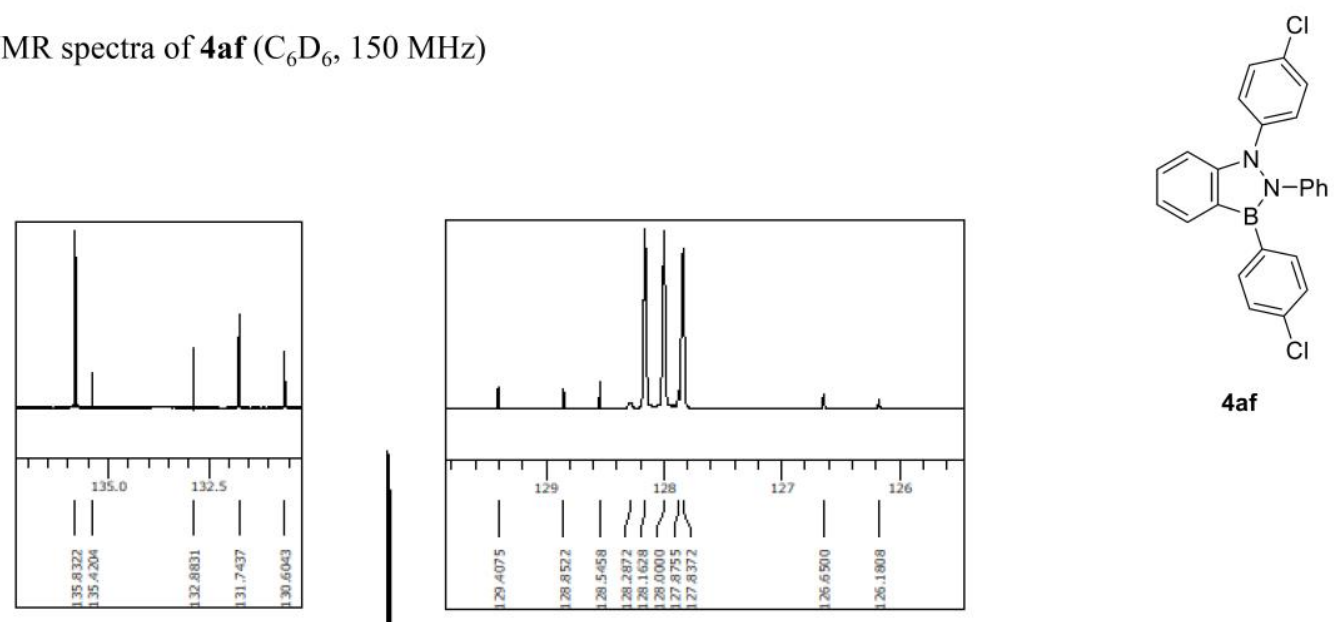

[

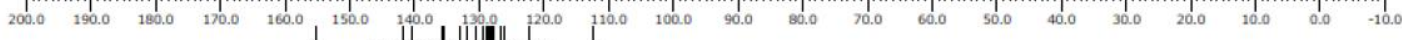

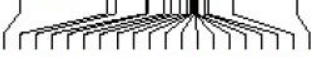

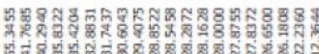

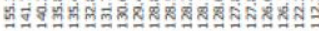


${ }^{11} \mathrm{~B}$ NMR spectra of $\mathbf{4 a f}\left(\mathrm{C}_{6} \mathrm{D}_{6}, 193 \mathrm{MHz}\right)$

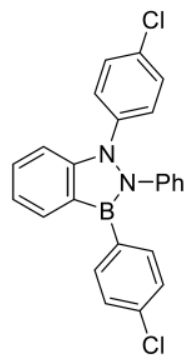

4 af

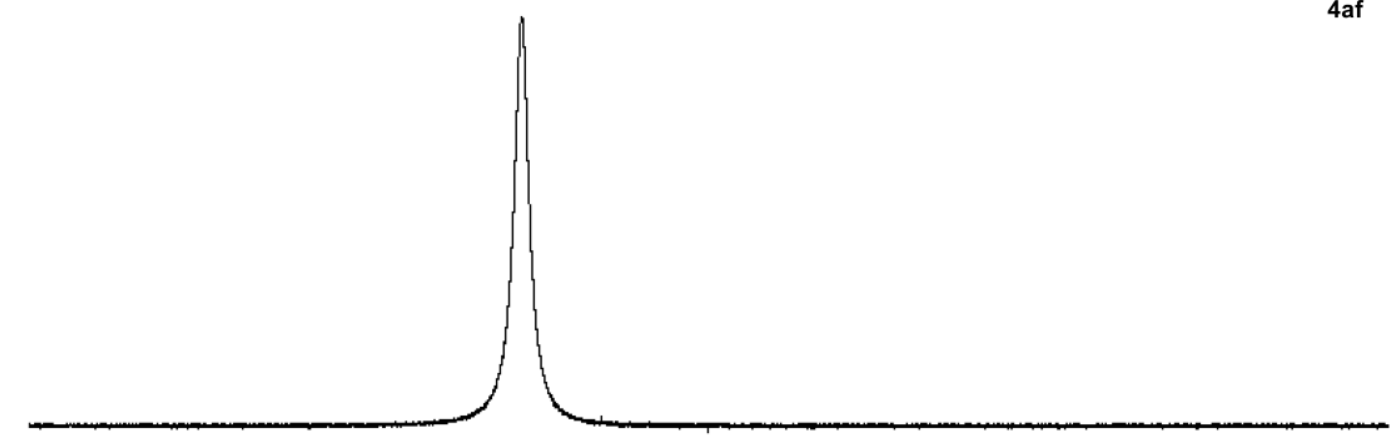

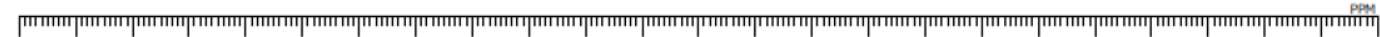
$\begin{array}{llllllllllllllllllllllllll}120.0 & 110.0 & 100.0 & 90.0 & 80.0 & 70.0 & 60.0 & 50.0 & 40.0 & 30.0 & 20.0 & 10.0 & 0.0 & -10.0 & -20.0 & -30.0 & -40.0 & -50.0 & -60.0 & -70.0 & -80.0 & -90.0 & -100.0 & -110.0 & -120.0\end{array}$

${ }^{1} \mathrm{H}$ NMR spectra of $\mathbf{4 a g}\left(\mathrm{C}_{6} \mathrm{D}_{6}, 400 \mathrm{MHz}\right)$

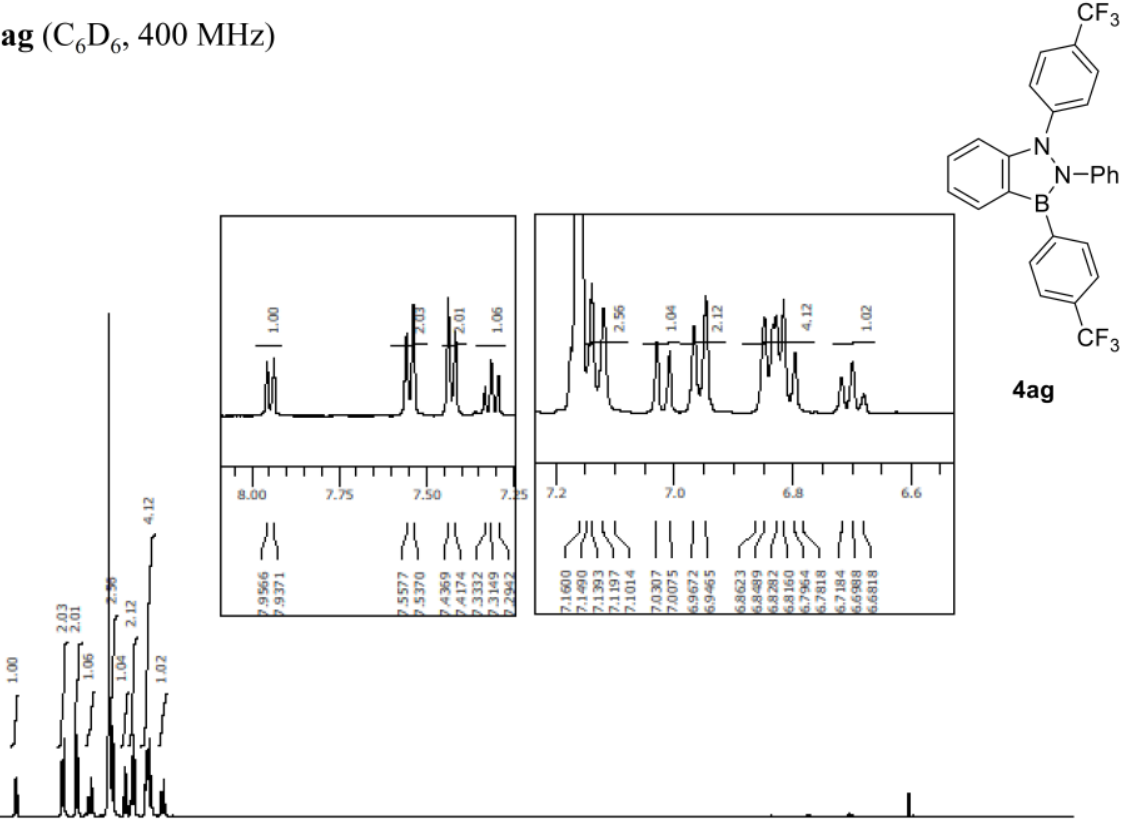

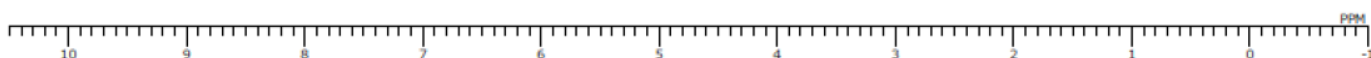

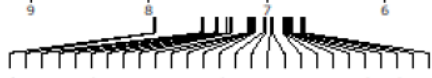

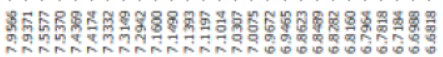


${ }^{13} \mathrm{C}$ NMR spectra of $\mathbf{4 a g}\left(\mathrm{CDCl}_{3}, 100 \mathrm{MHz}\right)$
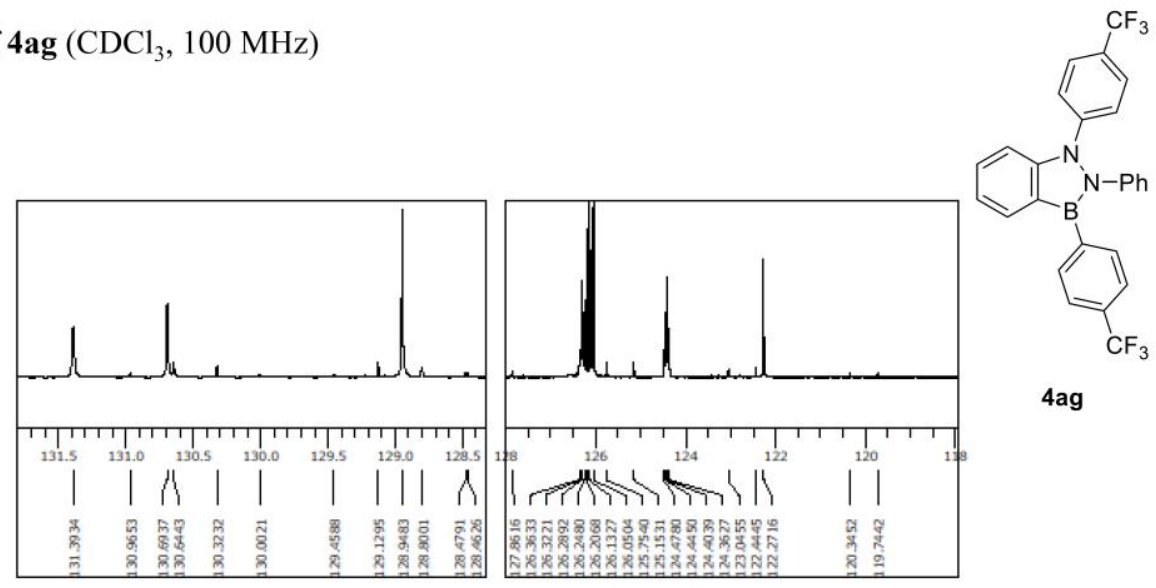

4ag
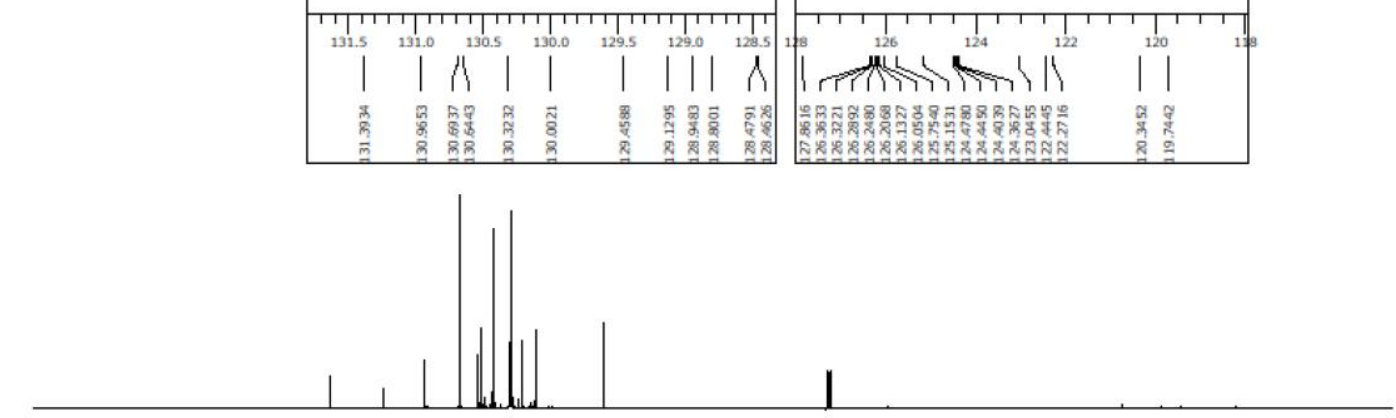

[

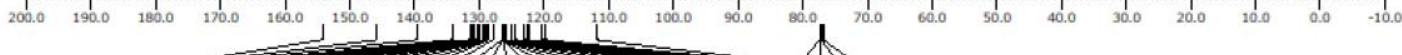

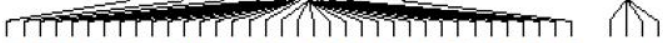

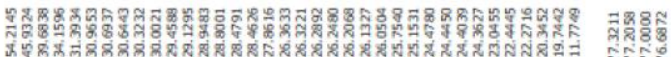

${ }^{11} \mathrm{~B}$ NMR spectra of $\mathbf{4 a g}\left(\mathrm{C}_{6} \mathrm{D}_{6}, 193 \mathrm{MHz}\right)$

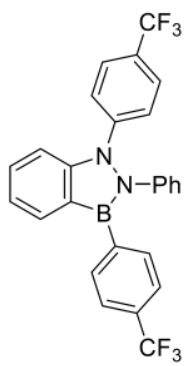

4ag

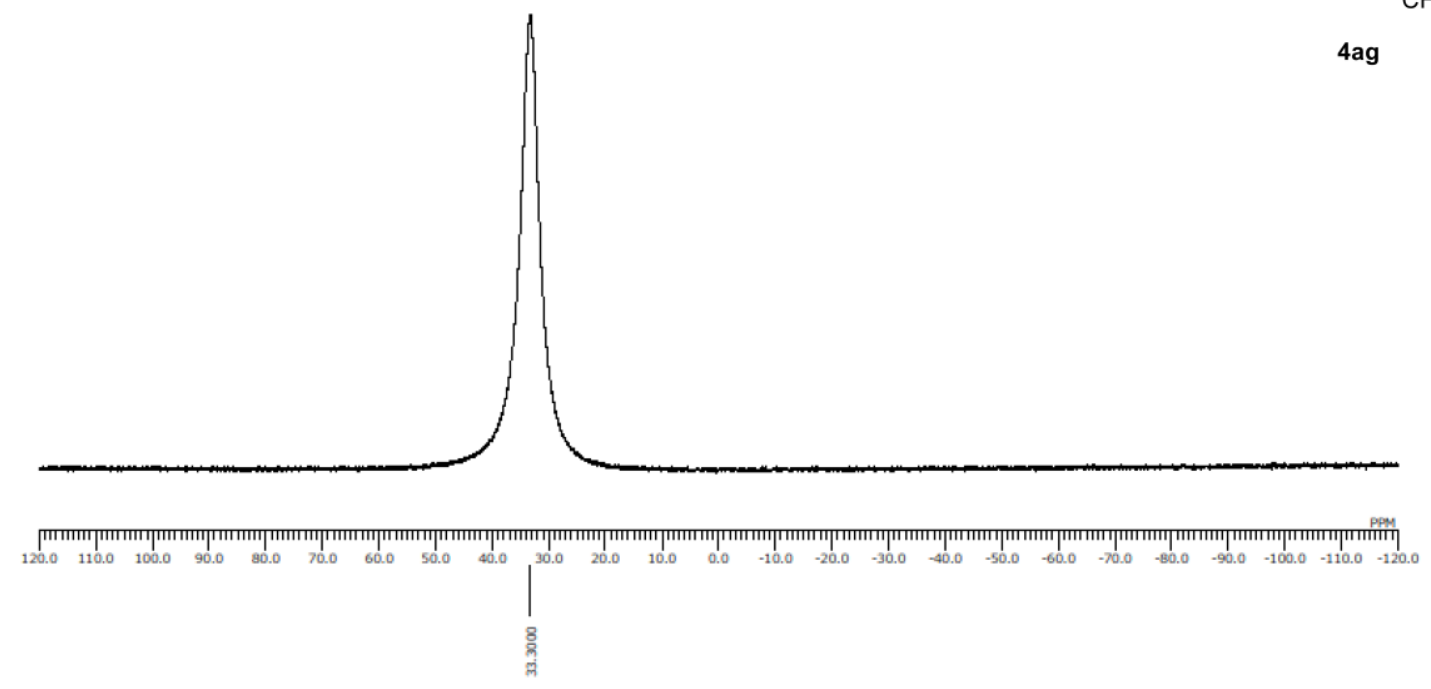


${ }^{1} \mathrm{H}$ NMR spectra of $\mathbf{4 b a}\left(\mathrm{CDCl}_{3}, 400 \mathrm{MHz}\right)$

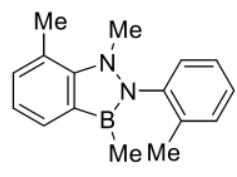

4ba

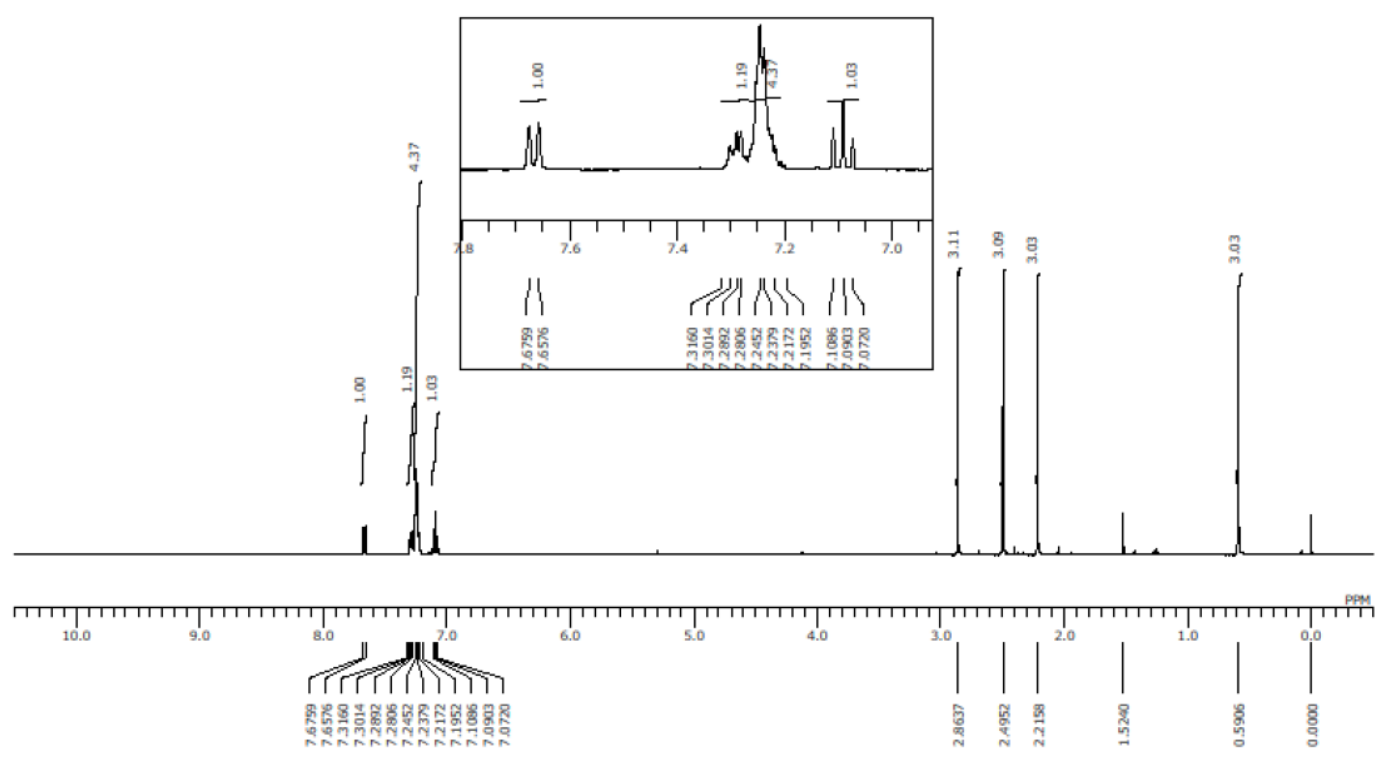

${ }^{13} \mathrm{C}$ NMR spectra of $\mathbf{4 b a}\left(\mathrm{C}_{6} \mathrm{D}_{6}, 100 \mathrm{MHz}\right)$
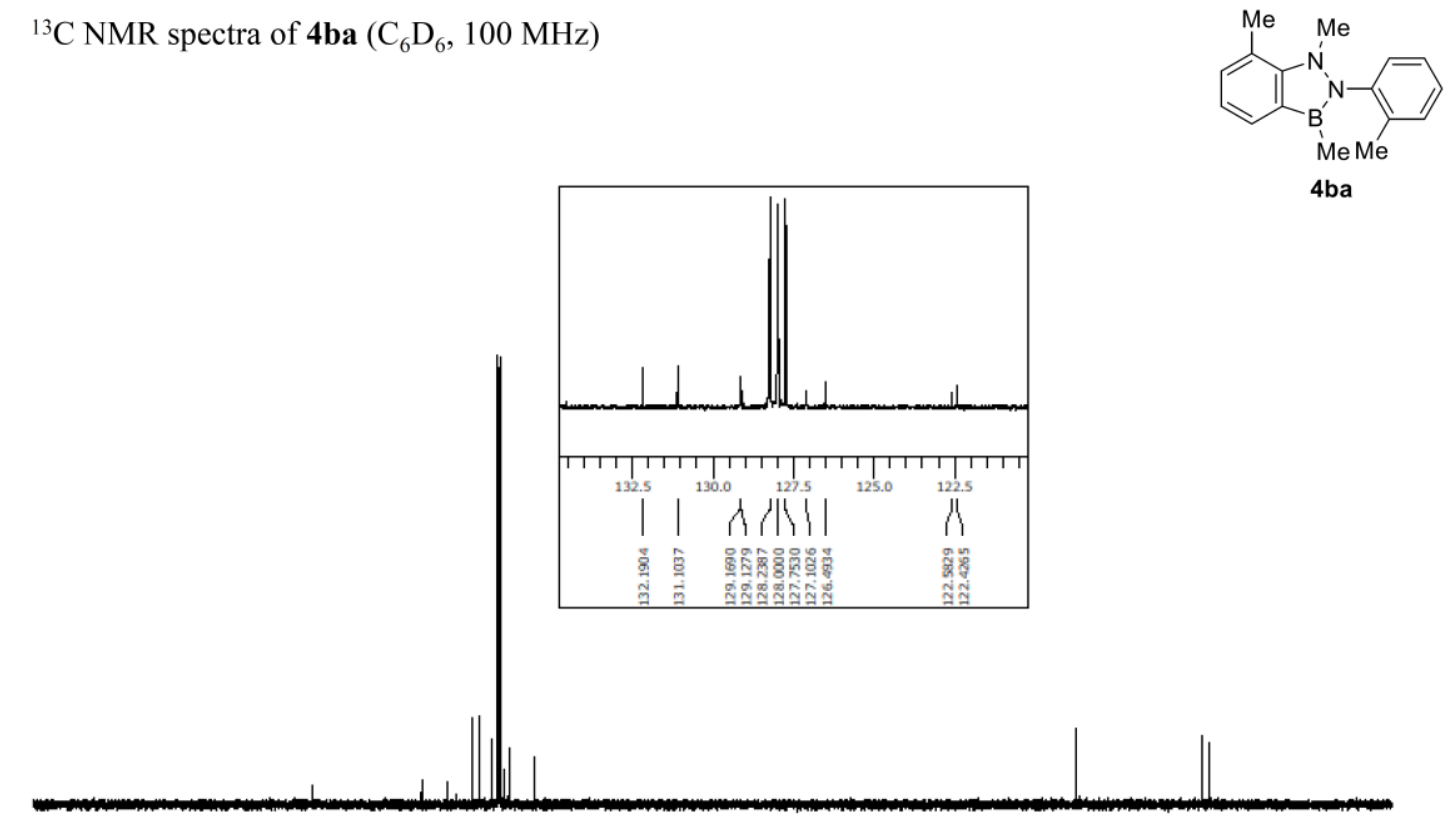

[

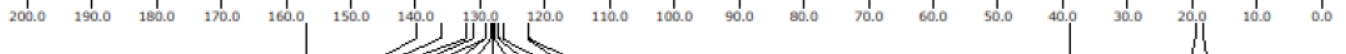

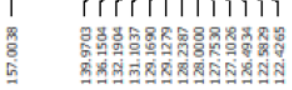

焉 
${ }^{11} \mathrm{~B}$ NMR spectra of $\mathbf{4 b a}\left(\mathrm{CDCl}_{3}, 193 \mathrm{MHz}\right)$

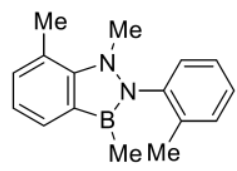

4ba

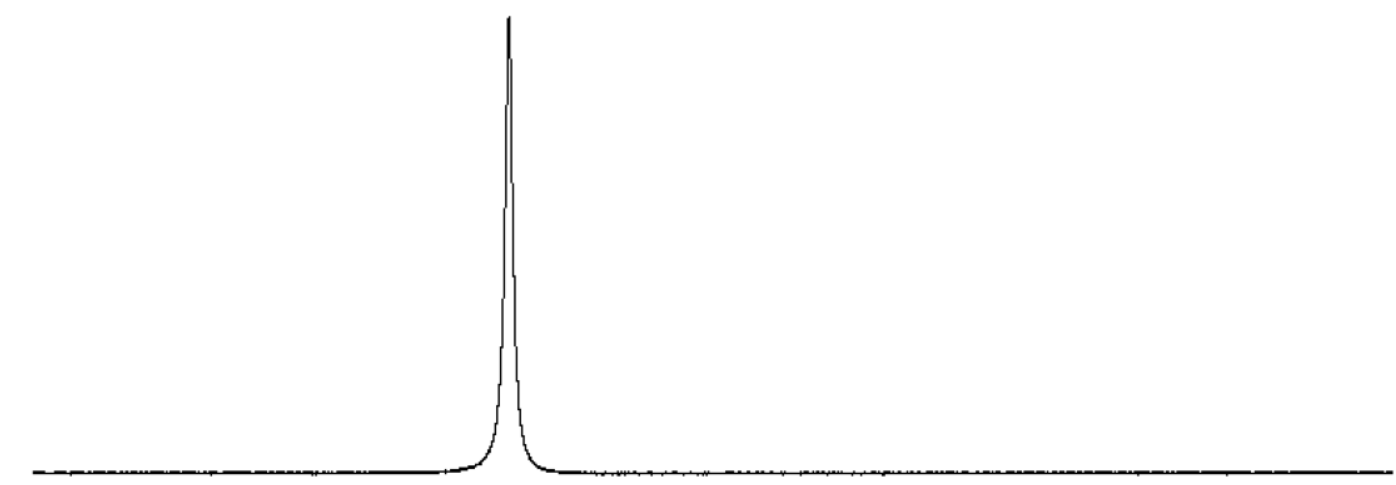

Г

${ }^{1} \mathrm{H}$ NMR spectra of $\mathbf{4} \mathbf{c a}\left(\mathrm{C}_{6} \mathrm{D}_{6}, 400 \mathrm{MHz}\right)$

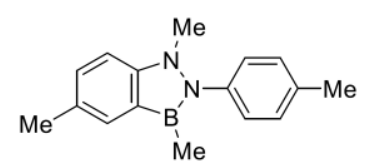

$4 \mathrm{ca}$

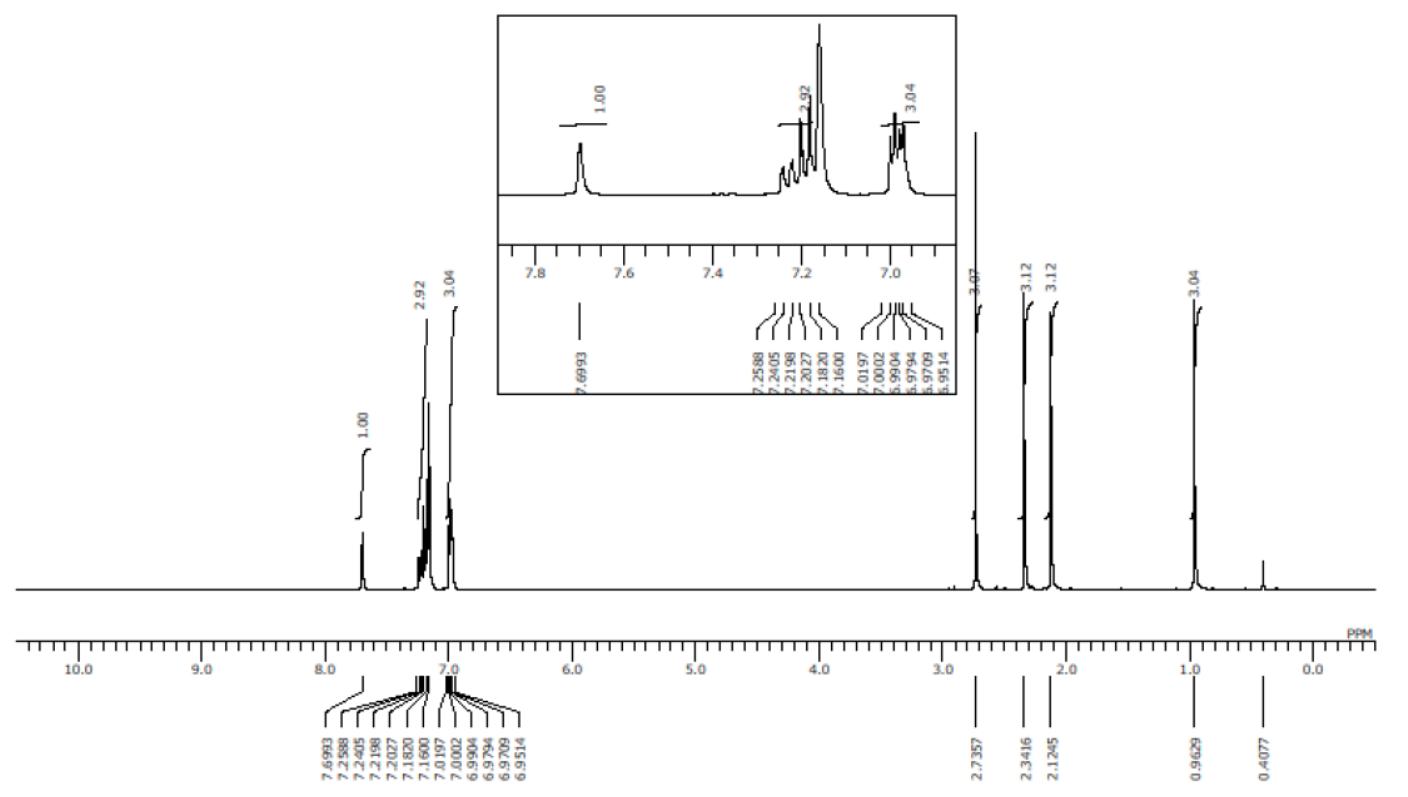


${ }^{13} \mathrm{C}$ NMR spectra of $\mathbf{4} \mathbf{c a}\left(\mathrm{CDCl}_{3}, 100 \mathrm{MHz}\right)$
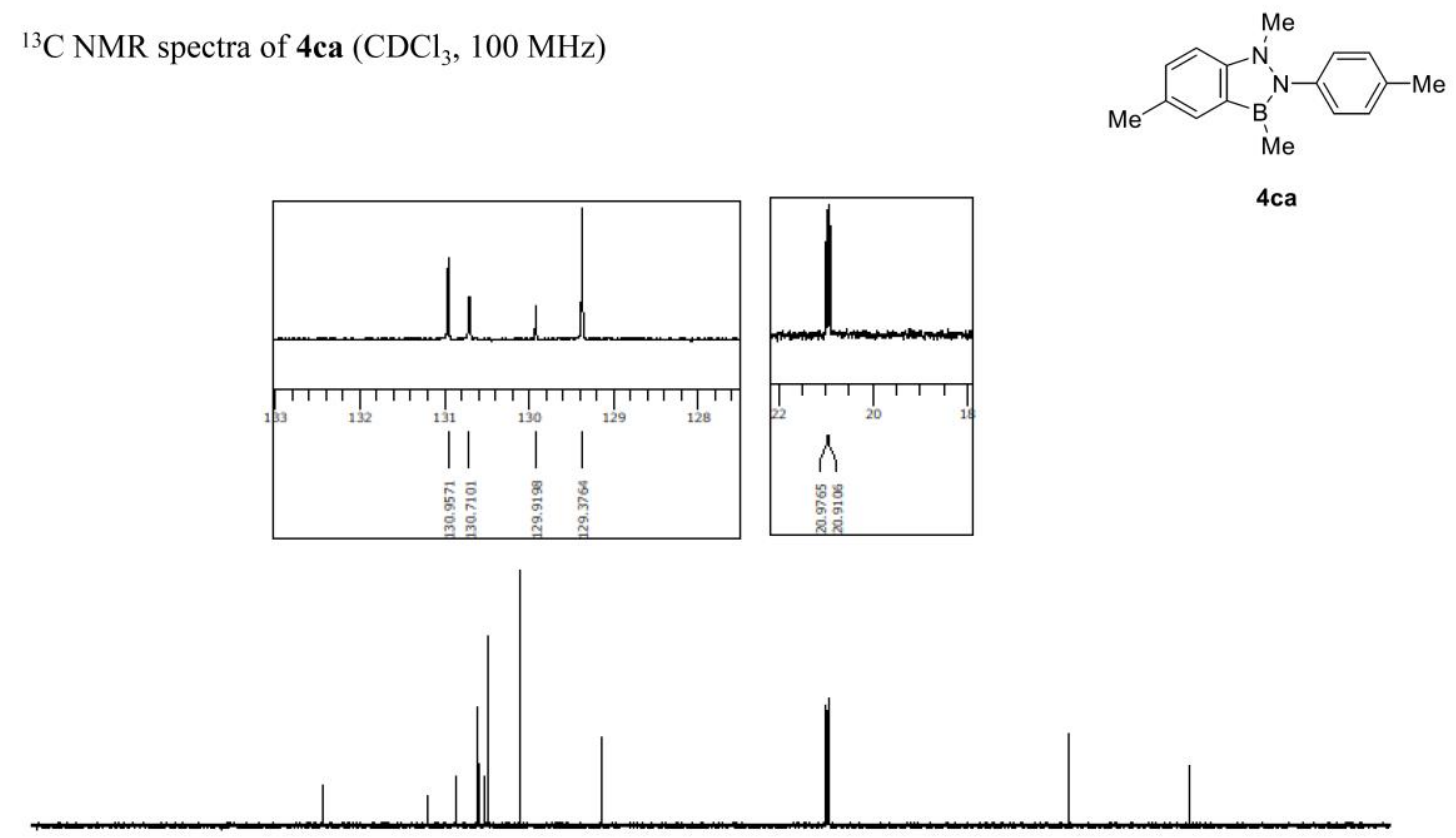

$4 \mathrm{ca}$

[

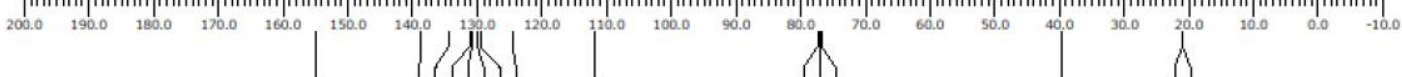

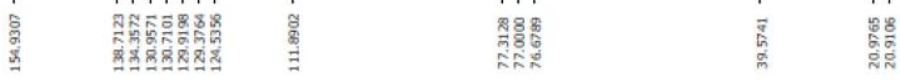

${ }^{11} \mathrm{~B}$ NMR spectra of $\mathbf{4 c a}\left(\mathrm{CDCl}_{3}, 193 \mathrm{MHz}\right)$

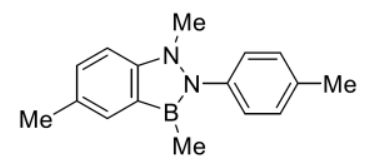

4ca

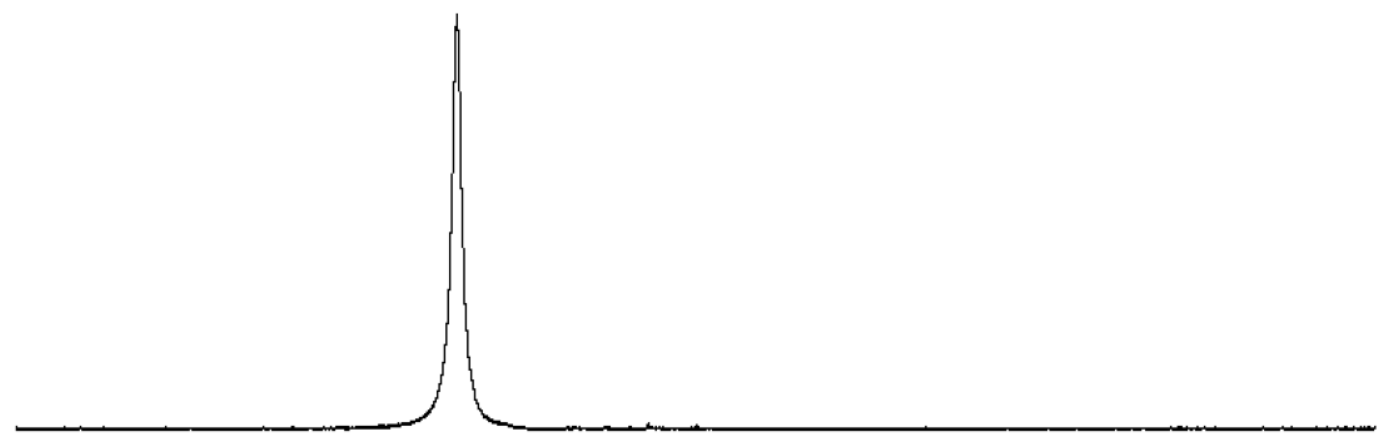

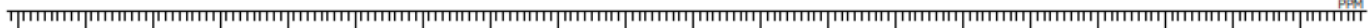

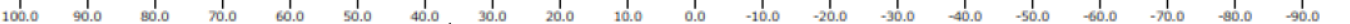
$\frac{1}{8}$ 
${ }^{1} \mathrm{H}$ NMR spectra of $4 \mathbf{c c}\left(\mathrm{CDCl}_{3}, 400 \mathrm{MHz}\right)$
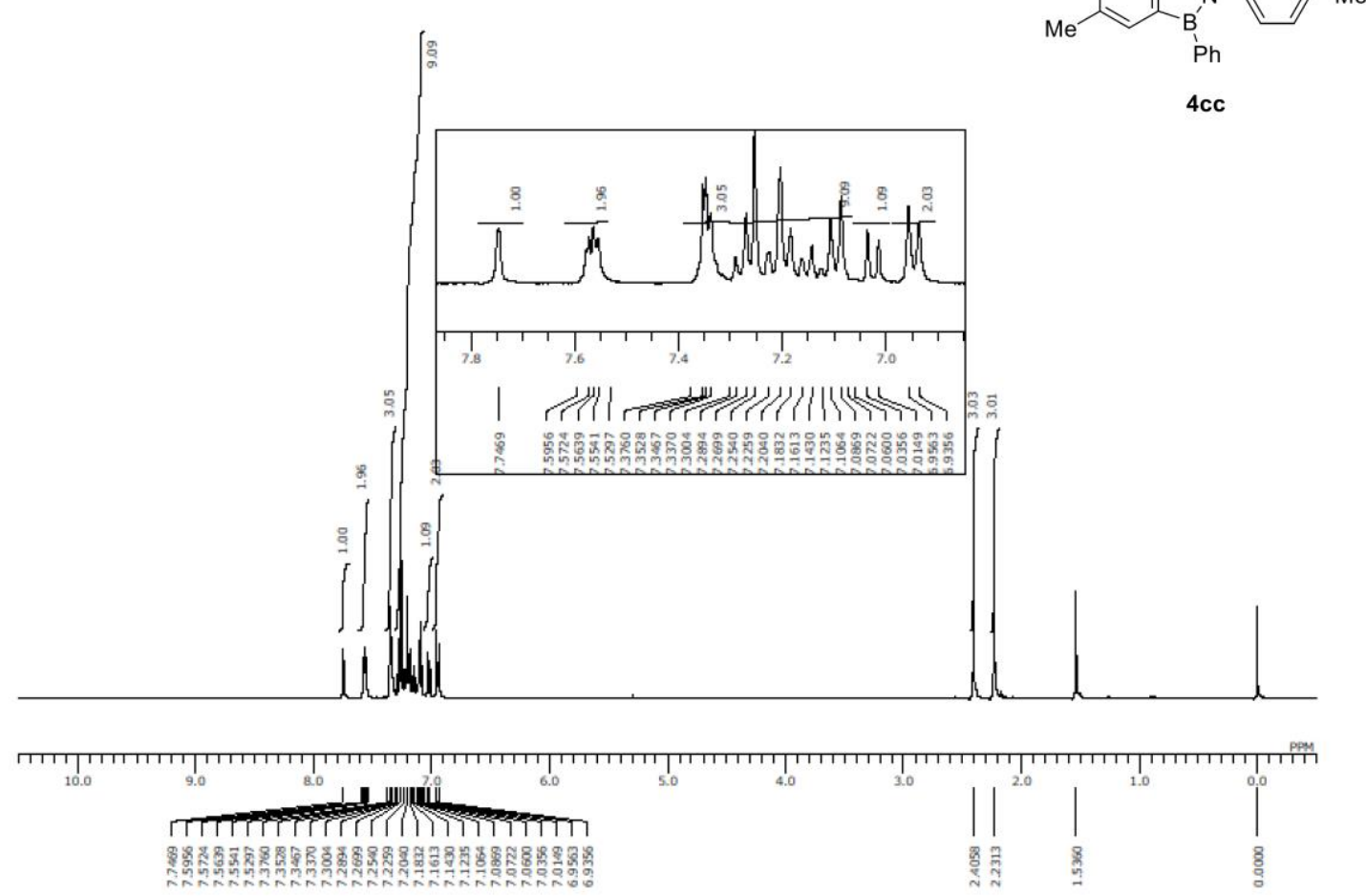

${ }^{13} \mathrm{C}$ NMR spectra of $\mathbf{4 c c}\left(\mathrm{CDCl}_{3}, 100 \mathrm{MHz}\right)$
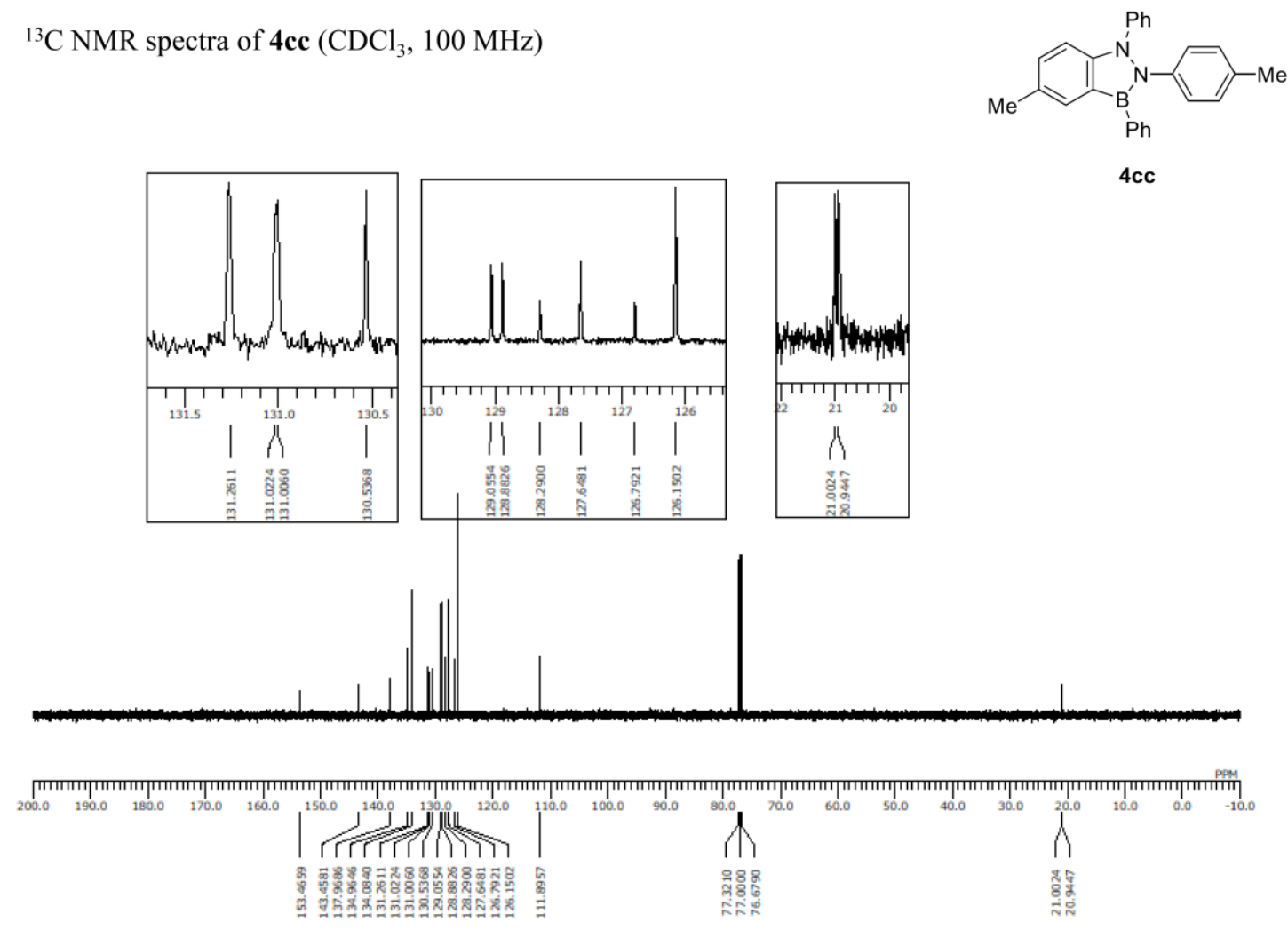

4cc 
${ }^{11} \mathrm{~B}$ NMR spectra of $\mathbf{4 c c}\left(\mathrm{CDCl}_{3}, 193 \mathrm{MHz}\right)$
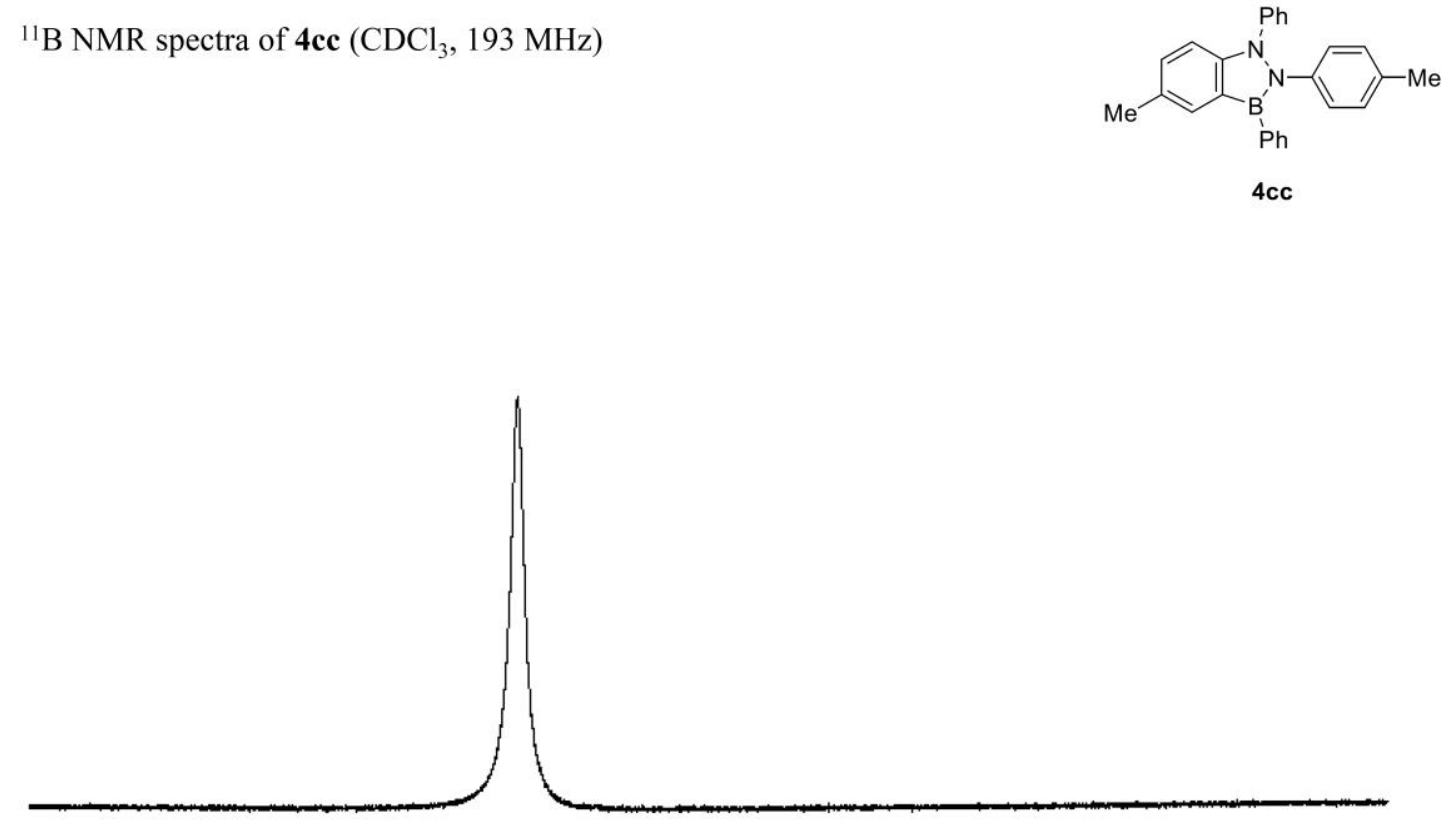

[ $\begin{array}{lllllllllllllllllllllllll}120.0 & 110.0 & 100.0 & 90.0 & 90.0 & 70.0 & 60.0 & 50.0 & 40.0 & 30.0 & 20.0 & 10.0 & 0.0 & -10.0 & -20.0 & -30.0 & -40.0 & -50.0 & -60.0 & -70.0 & -80.0 & -90.0 & -100.0 & -110.0 & -120.0\end{array}$

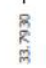

${ }^{1} \mathrm{H}$ NMR spectra of $\mathbf{4 d a}\left(\mathrm{CDCl}_{3}, 400 \mathrm{MHz}\right)$
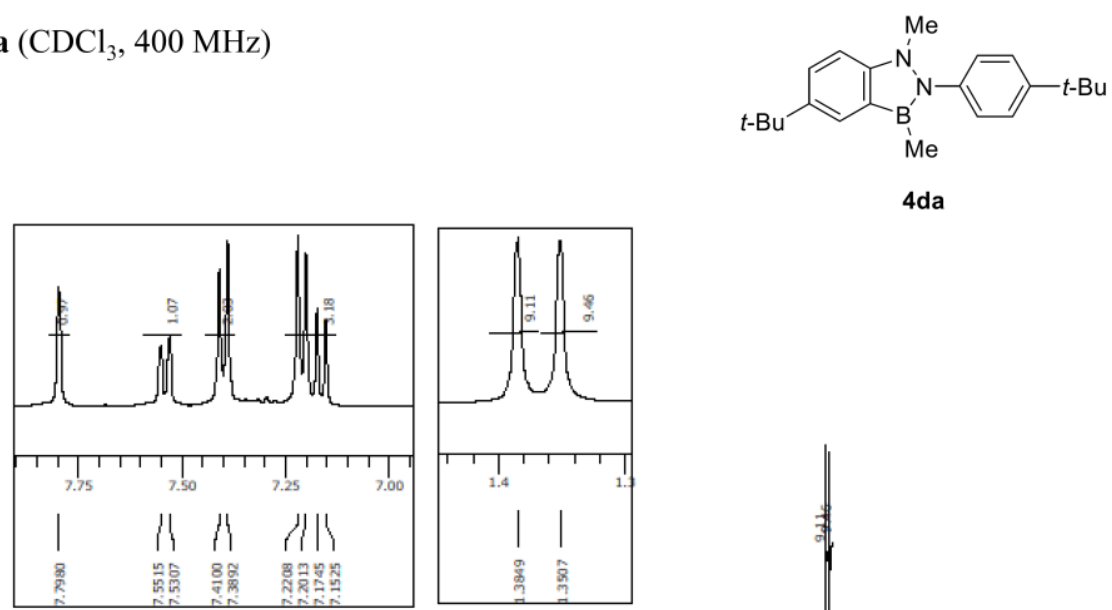

4da

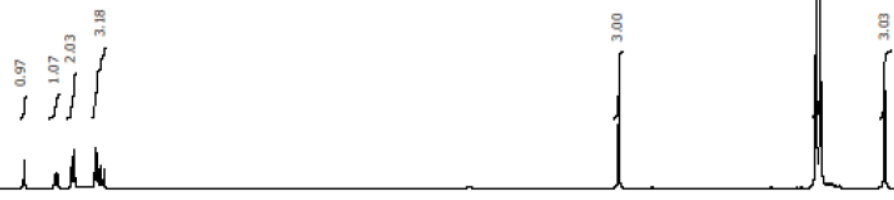

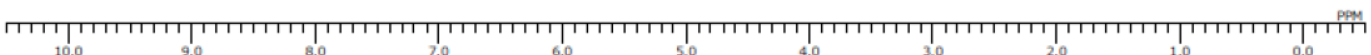

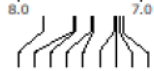

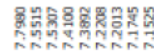

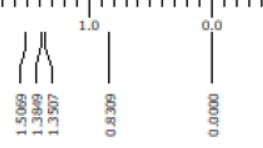


${ }^{13} \mathrm{C}$ NMR spectra of $\mathbf{4 d a}\left(\mathrm{C}_{6} \mathrm{D}_{6}, 100 \mathrm{MHz}\right)$
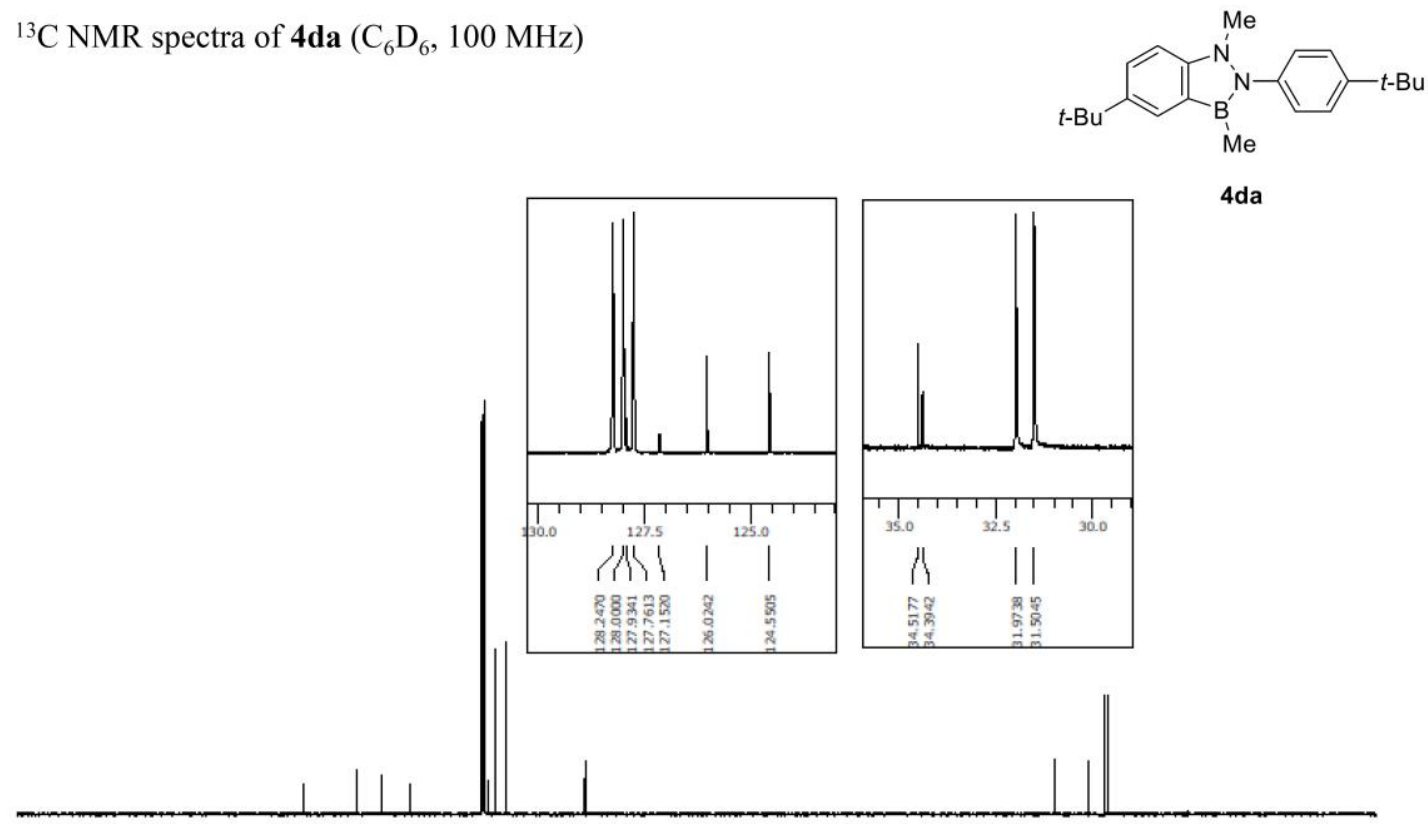

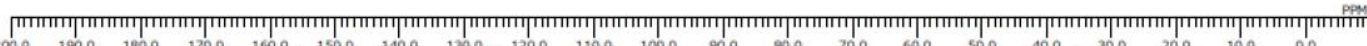

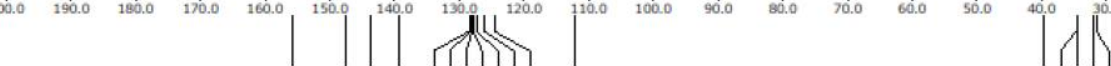

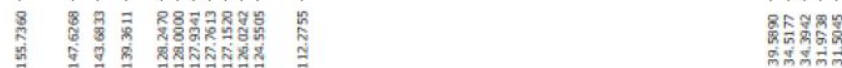

${ }^{11} \mathrm{~B}$ NMR spectra of $\mathbf{4 d a}\left(\mathrm{CDCl}_{3}, 193 \mathrm{MHz}\right)$

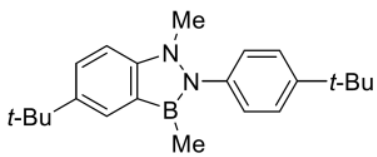

4da

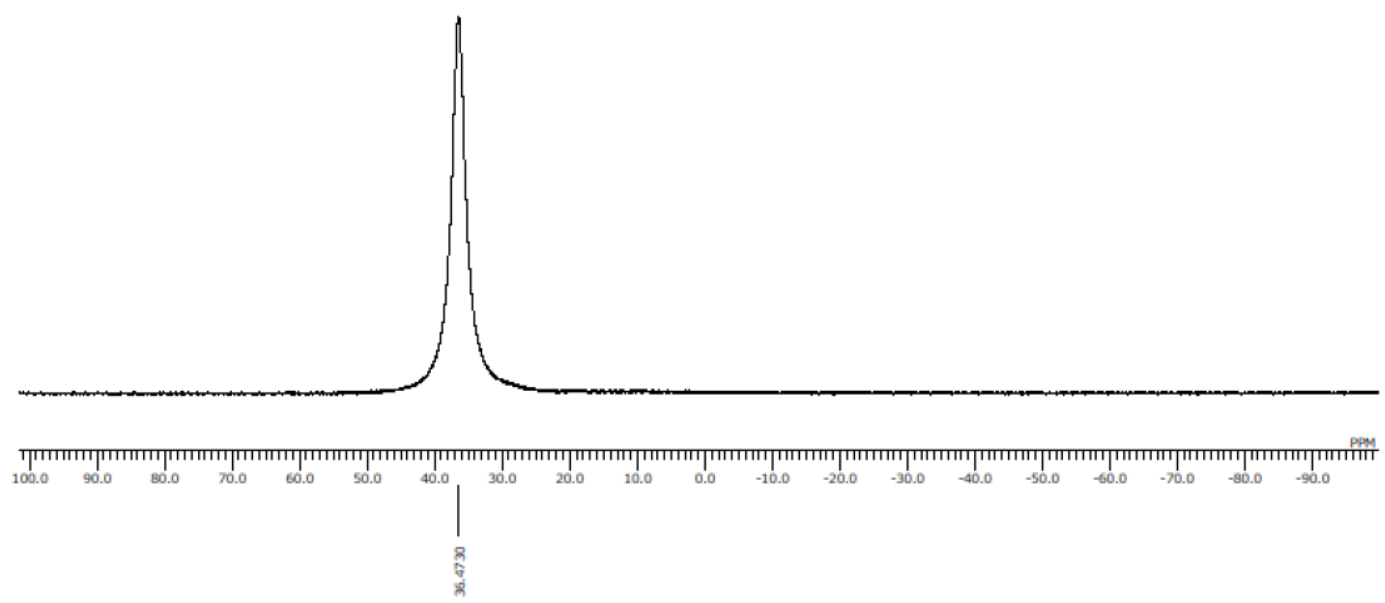


${ }^{1} \mathrm{H}$ NMR spectra of $\mathbf{4 d c}\left(\mathrm{CDCl}_{3}, 400 \mathrm{MHz}\right)$
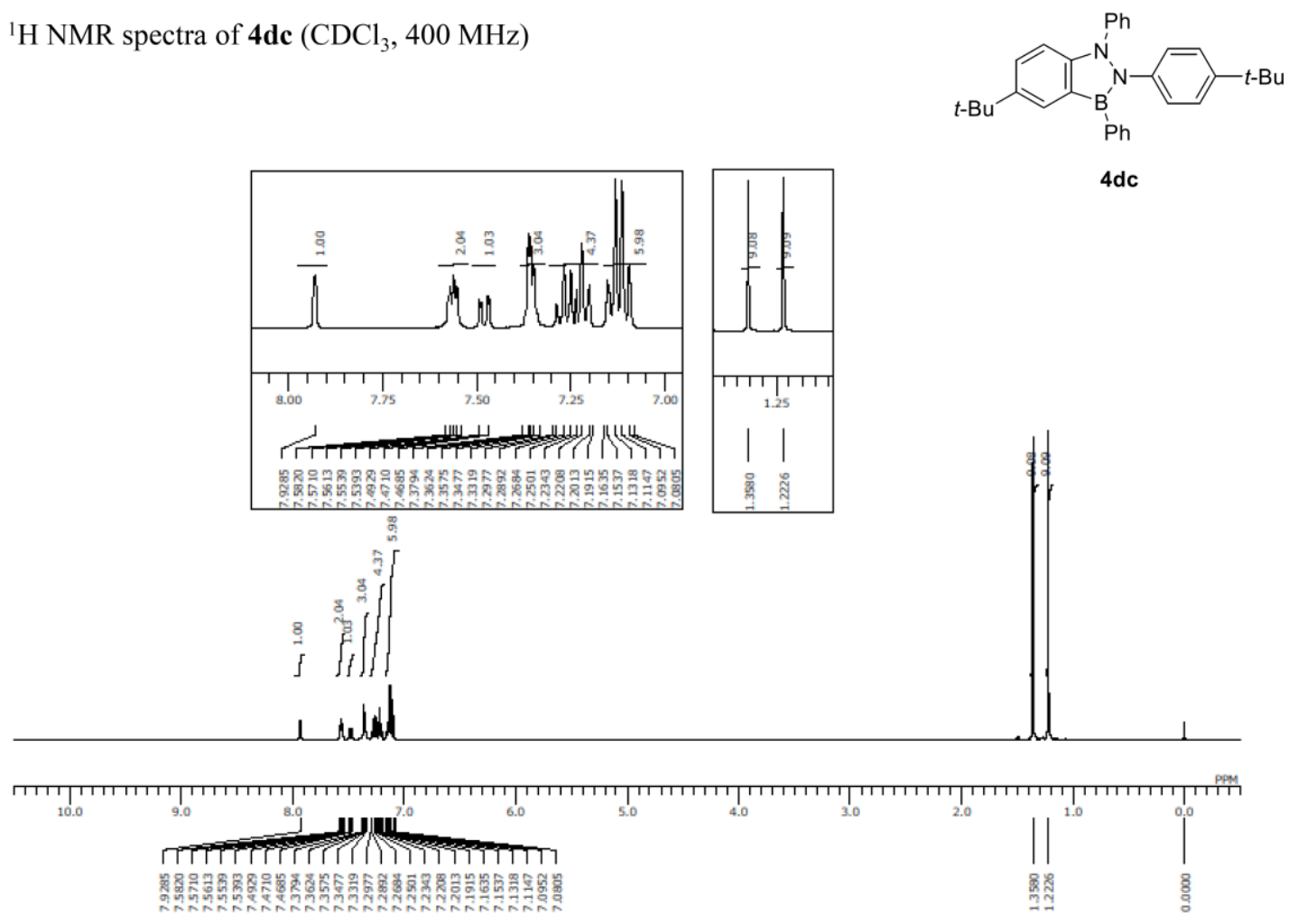

${ }^{13} \mathrm{C}$ NMR spectra of $\mathbf{4 d c}\left(\mathrm{CDCl}_{3}, 100 \mathrm{MHz}\right)$
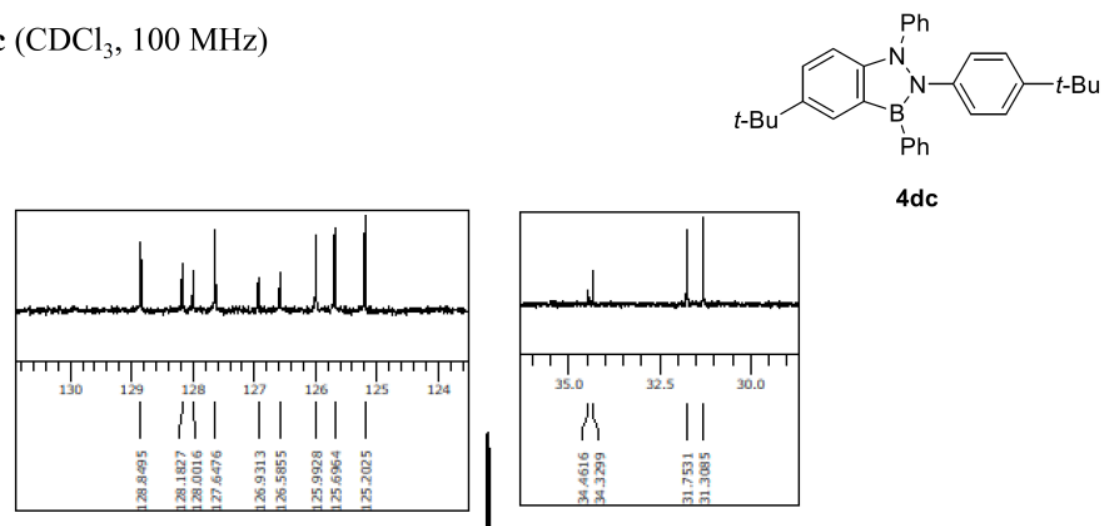

4dc

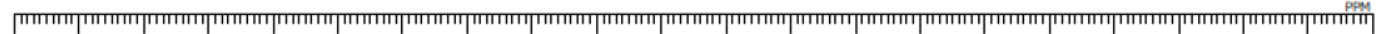

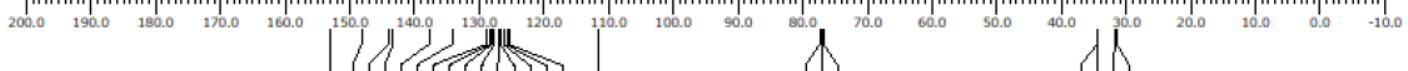

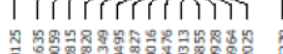

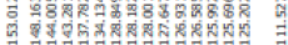


${ }^{11} \mathrm{~B}$ NMR spectra of $\mathbf{4 d c}\left(\mathrm{CDCl}_{3}, 193 \mathrm{MHz}\right)$

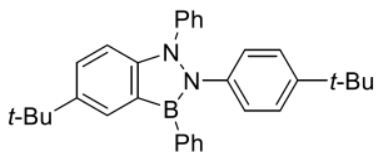

4dc

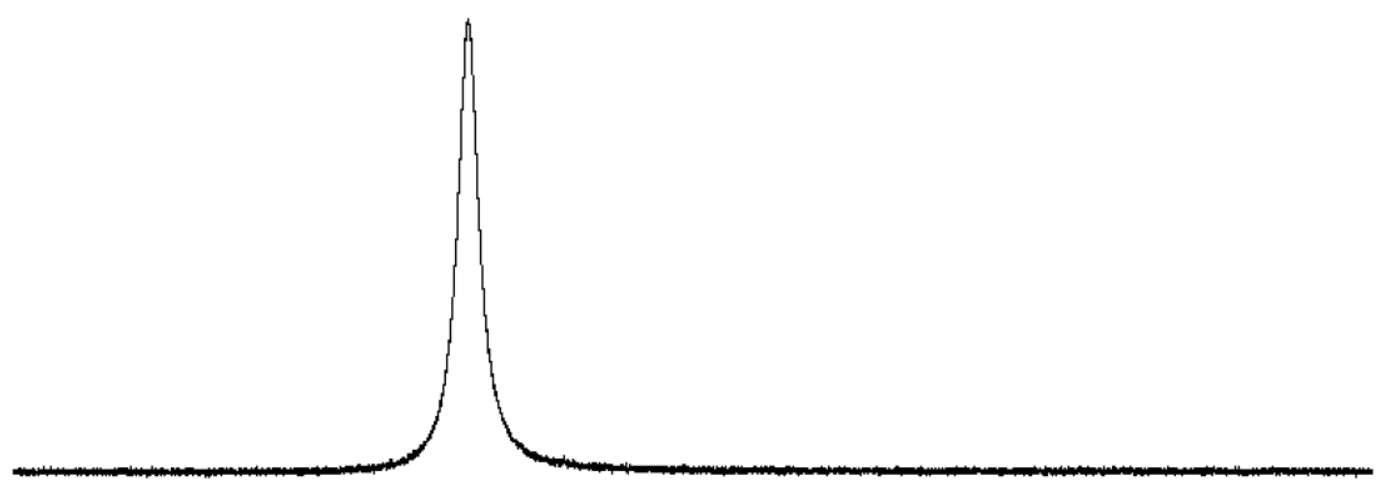

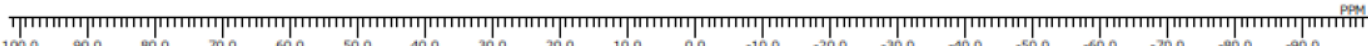

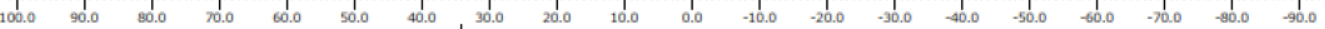

${ }^{1} \mathrm{H}$ NMR spectra of $\mathbf{4 e a}\left(\mathrm{CDCl}_{3}, 400 \mathrm{MHz}\right)$

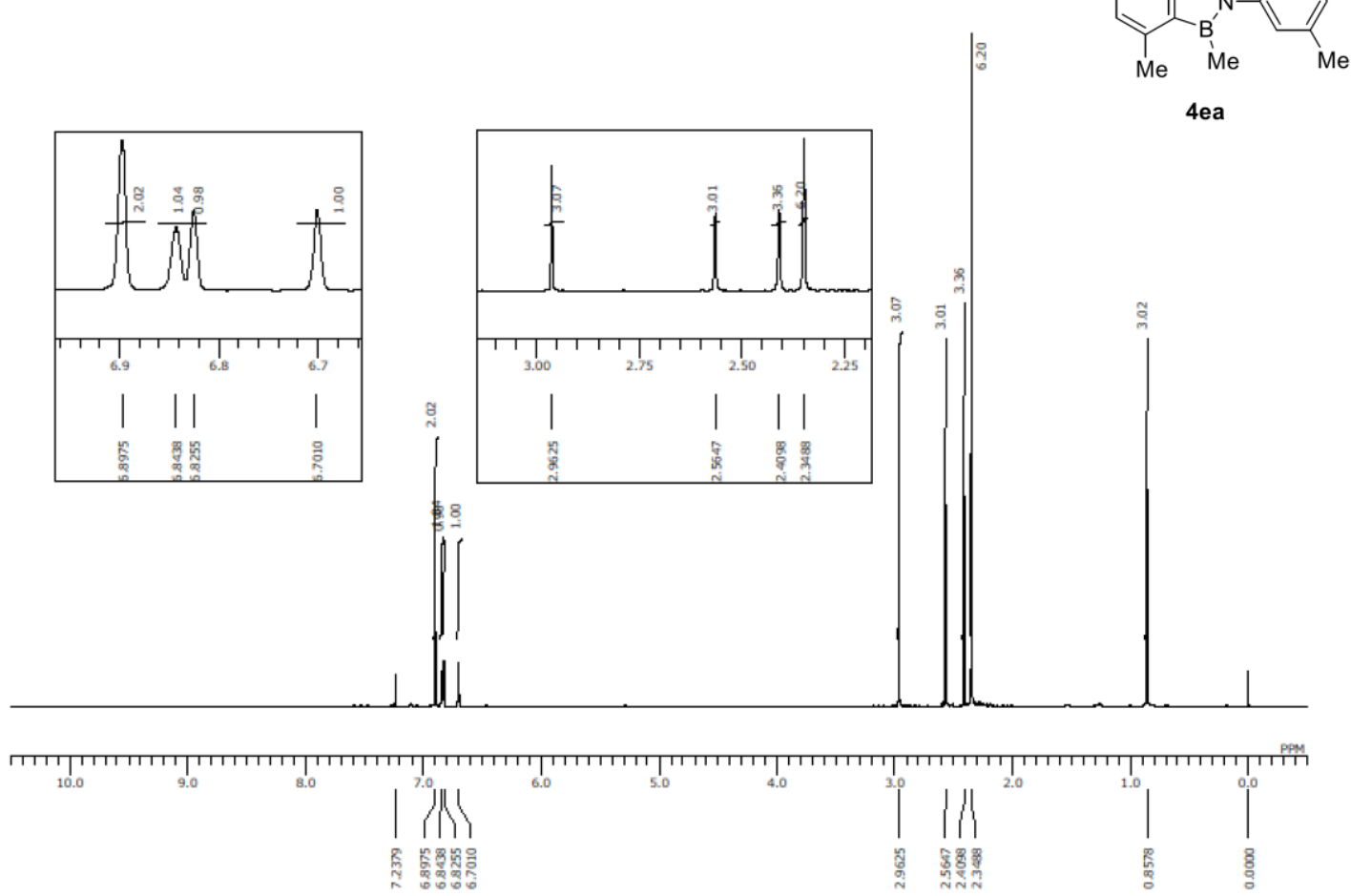


${ }^{13} \mathrm{C}$ NMR spectra of $4 \mathrm{ea}\left(\mathrm{C}_{6} \mathrm{D}_{6}, 100 \mathrm{MHz}\right)$
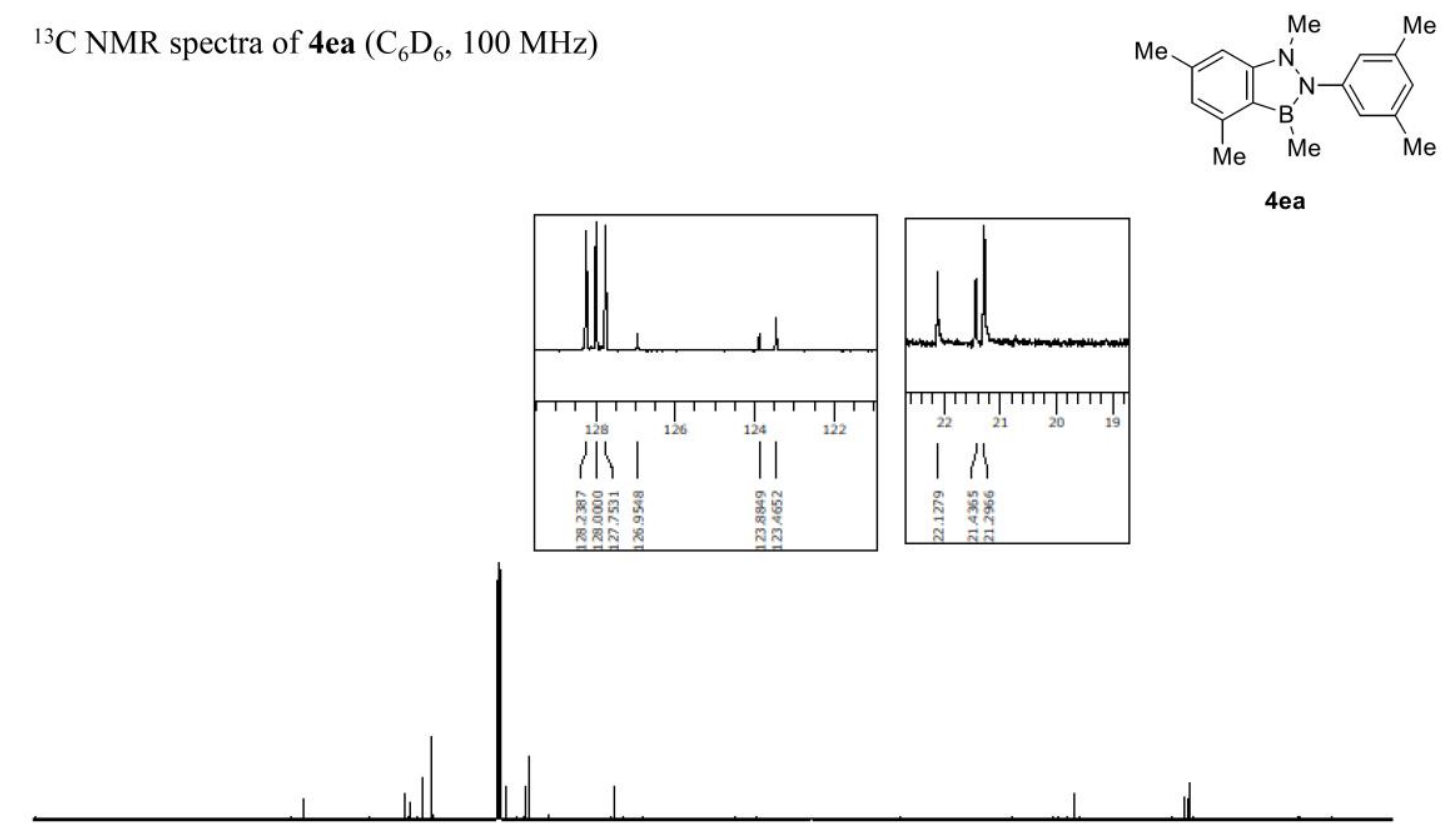

4ea

(

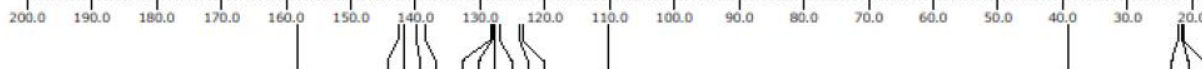

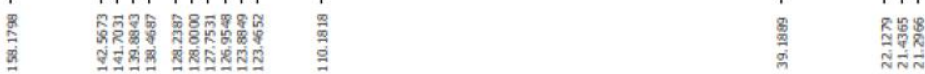

${ }^{11} \mathrm{~B}$ NMR spectra of $4 \mathrm{ea}\left(\mathrm{CDCl}_{3}, 193 \mathrm{MHz}\right)$

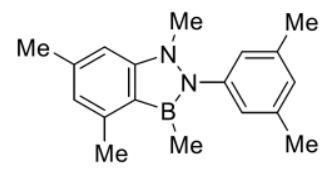

4 ea

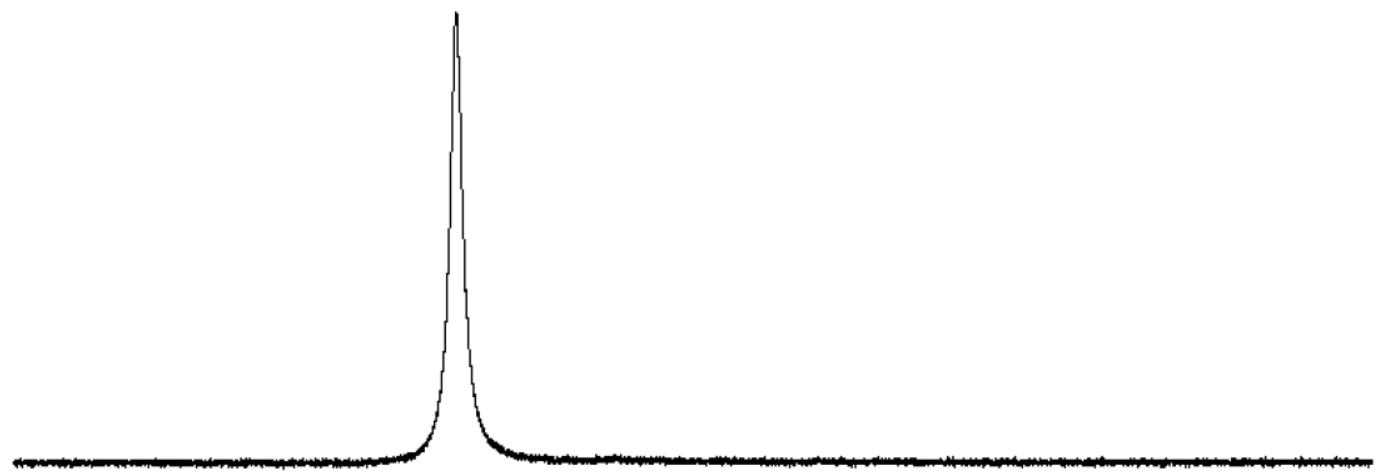

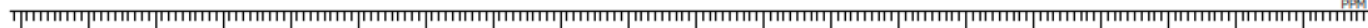
T100 
${ }^{1} \mathrm{H}$ NMR spectra of $\mathbf{4 f a}\left(\mathrm{C}_{6} \mathrm{D}_{6}, 400 \mathrm{MHz}\right)$
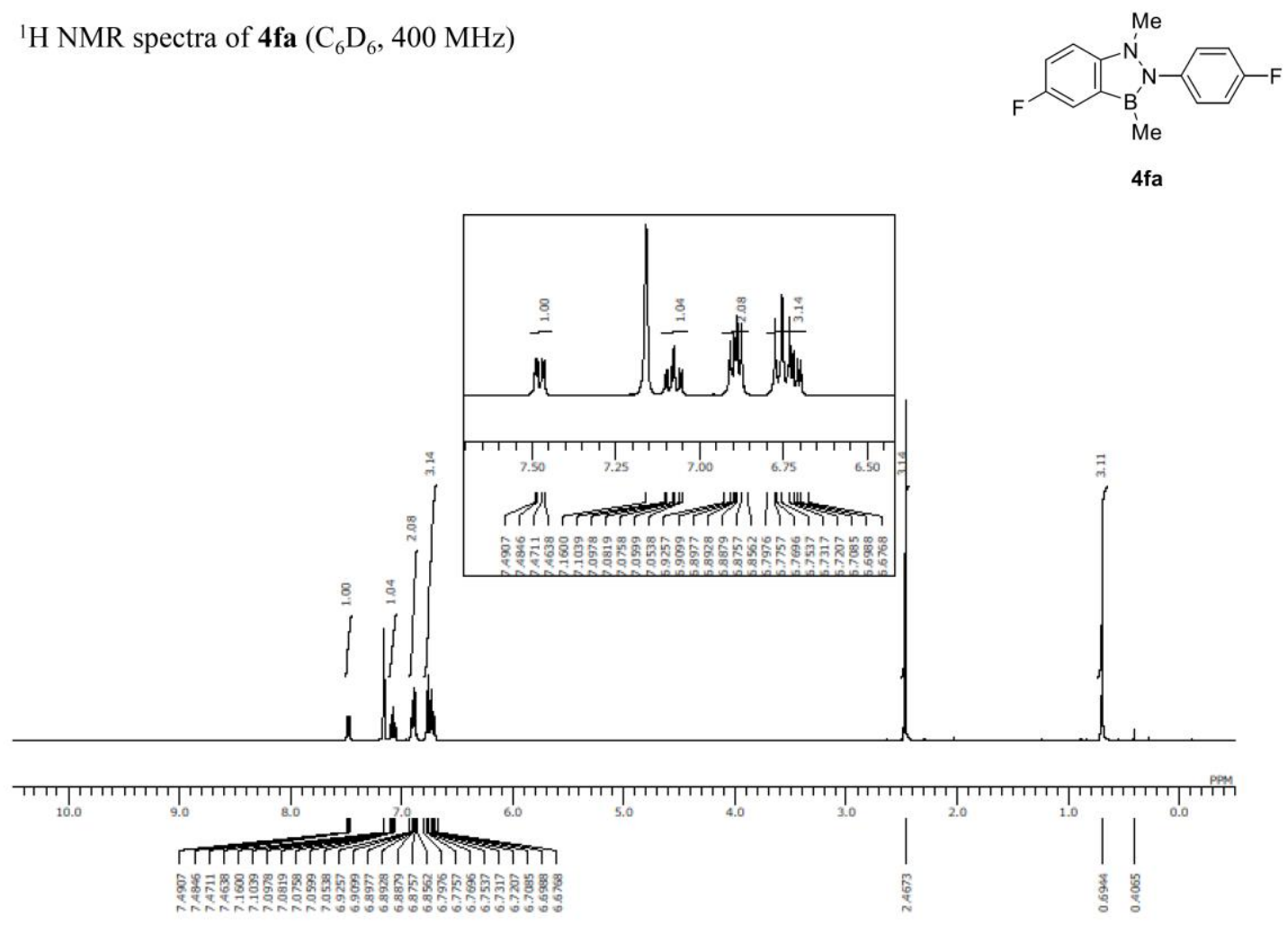

${ }^{13} \mathrm{C}$ NMR spectra of $\mathbf{4 f a}\left(\mathrm{CDCl}_{3}, 150 \mathrm{MHz}\right)$
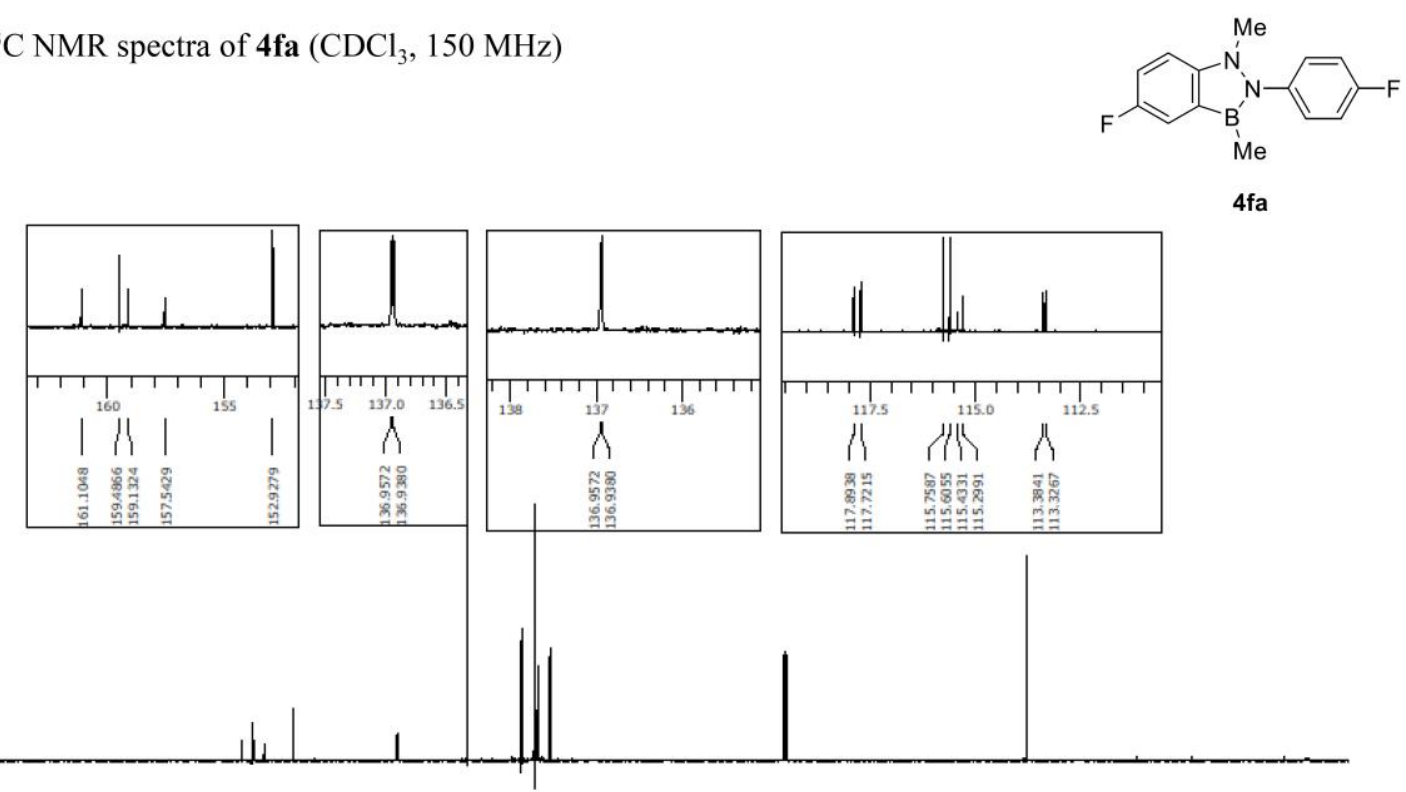

[ $\begin{array}{lllllllllllllll} & 1000 & 200 & 100 & 0\end{array}$

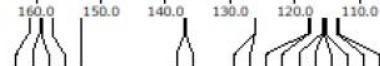

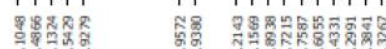
M

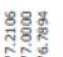


${ }^{11} \mathrm{~B}$ NMR spectra of $\mathbf{4 f a}\left(\mathrm{CDCl}_{3}, 193 \mathrm{MHz}\right)$
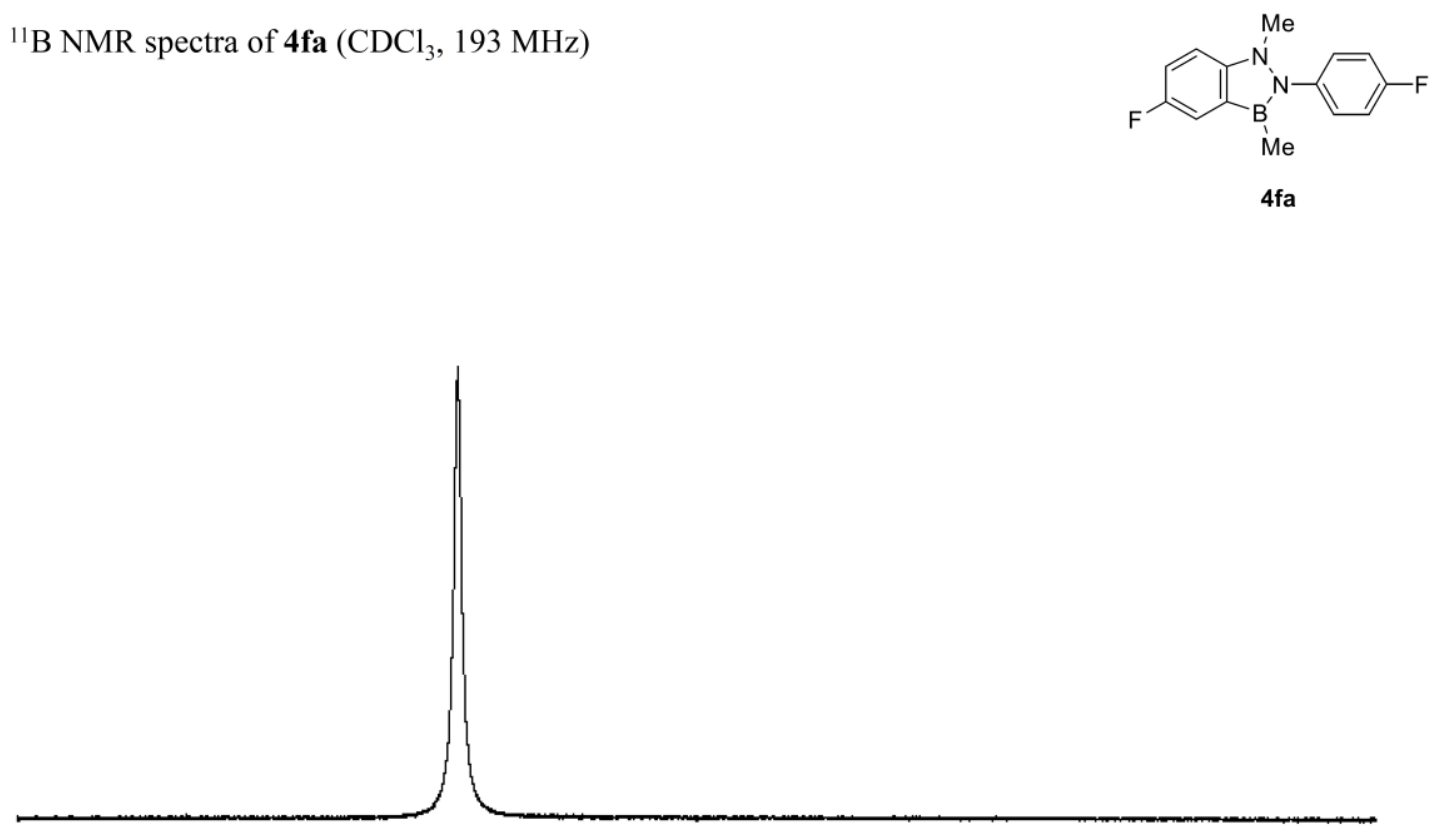

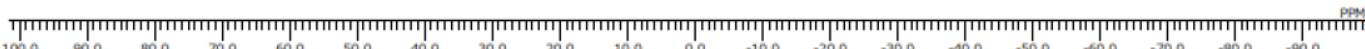
$\begin{array}{llllllllllllllllllll}100.0 & 90.0 & 80.0 & 70.0 & 60.0 & 50.0 & 40.0 & 30.0 & 20.0 & 10.0 & 0.0 & -10.0 & -20.0 & -30.0 & -40.0 & -50.0 & -60.0 & -70.0 & -80.0 & -90.0\end{array}$

${ }^{1} \mathrm{H}$ NMR spectra of $\mathbf{4 f c}\left(\mathrm{C}_{6} \mathrm{D}_{6}, 400 \mathrm{MHz}\right)$
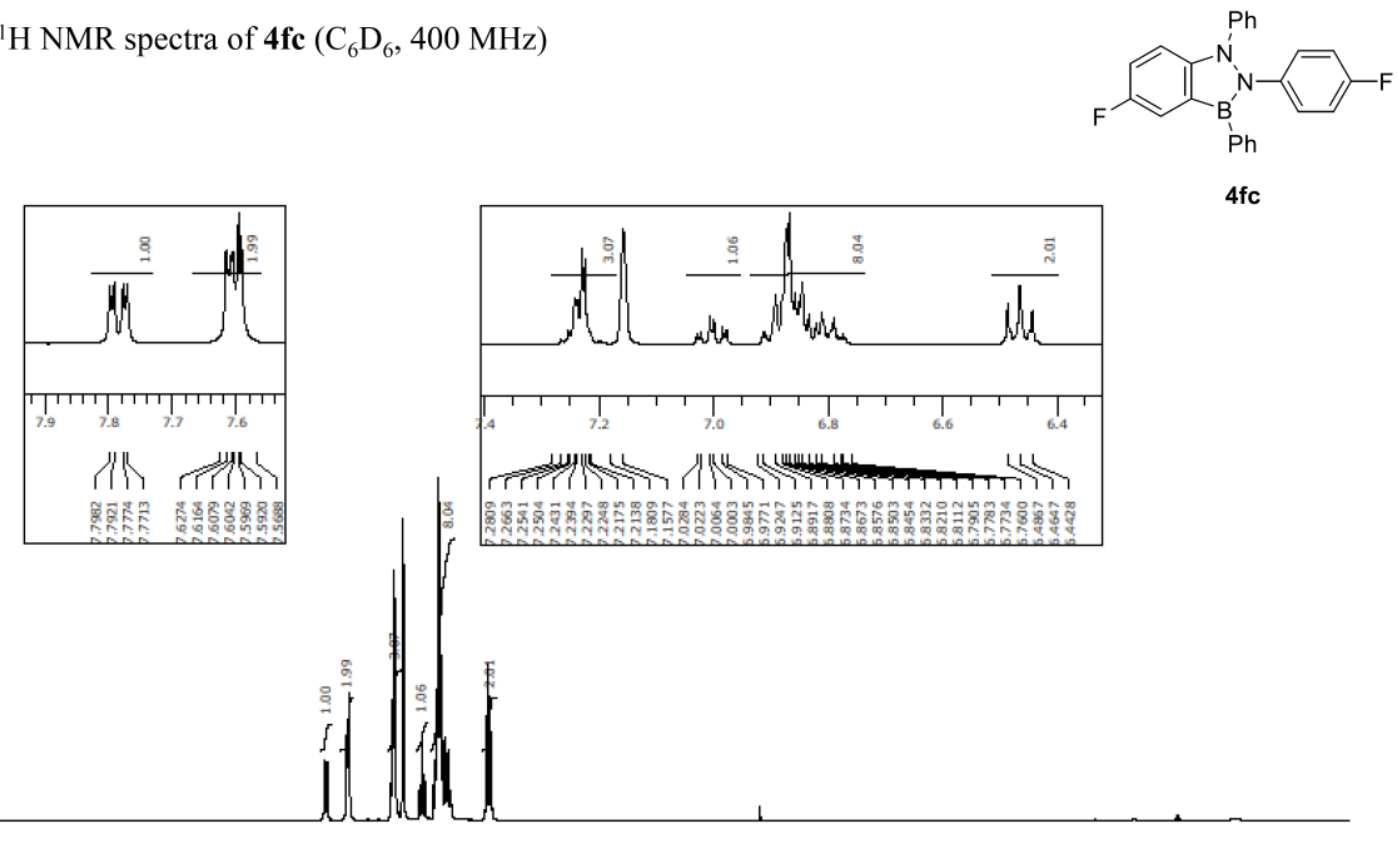

$4 \mathrm{fc}$

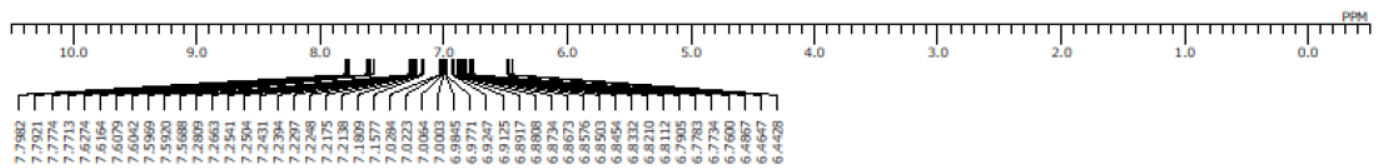


${ }^{13} \mathrm{C}$ NMR spectra of $\mathbf{4 f c}\left(\mathrm{CDCl}_{3}, 100 \mathrm{MHz}\right)$
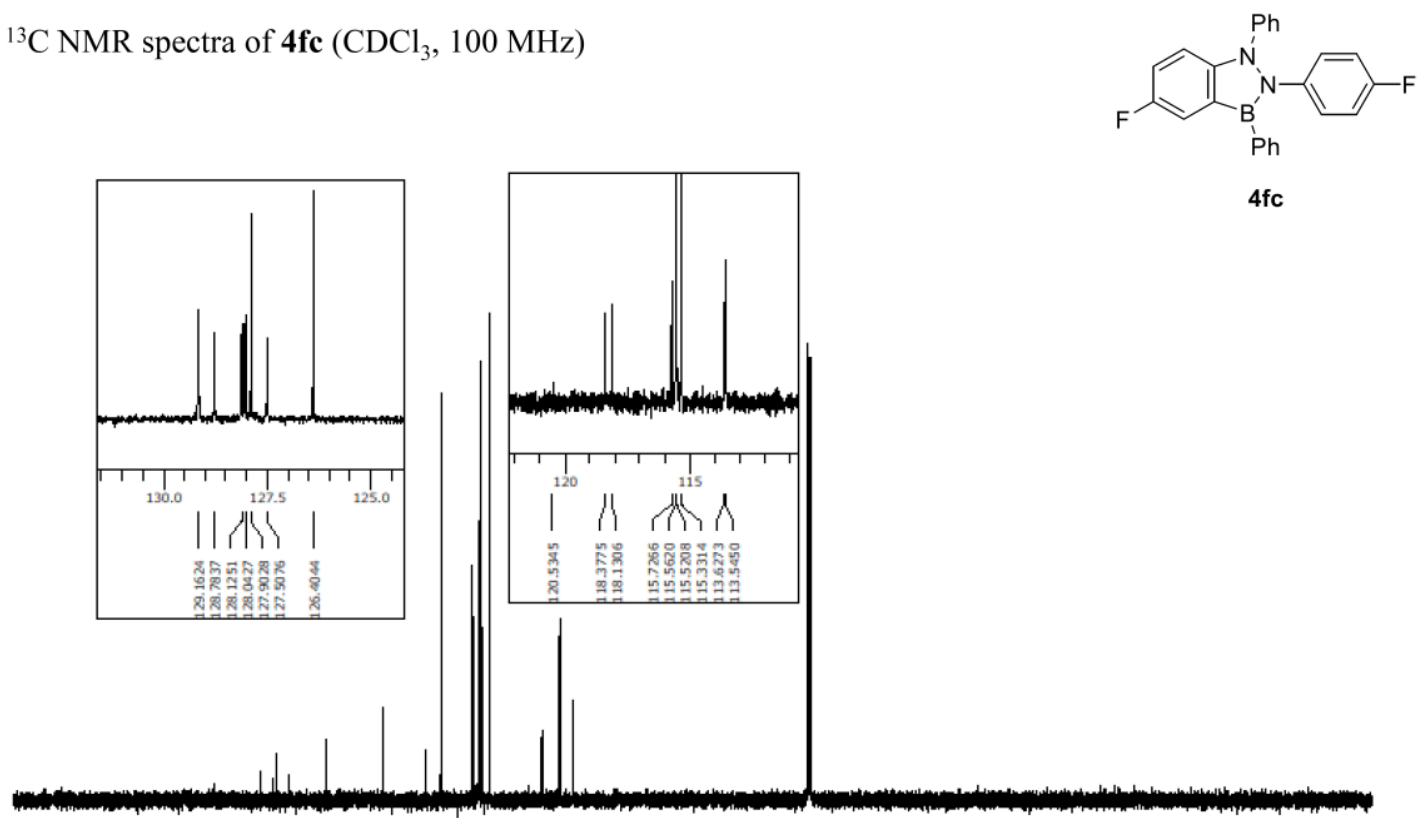

[

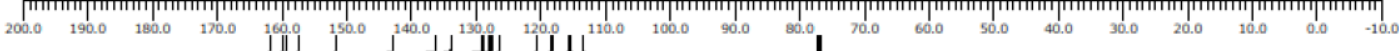

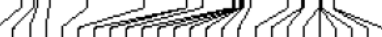

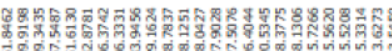

円)

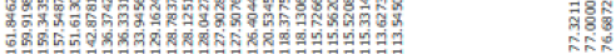

${ }^{11} \mathrm{~B}$ NMR spectra of $\mathbf{4 f c}\left(\mathrm{C}_{6} \mathrm{D}_{6}, 193 \mathrm{MHz}\right)$

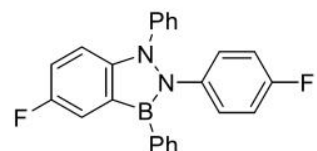

$4 \mathrm{fc}$

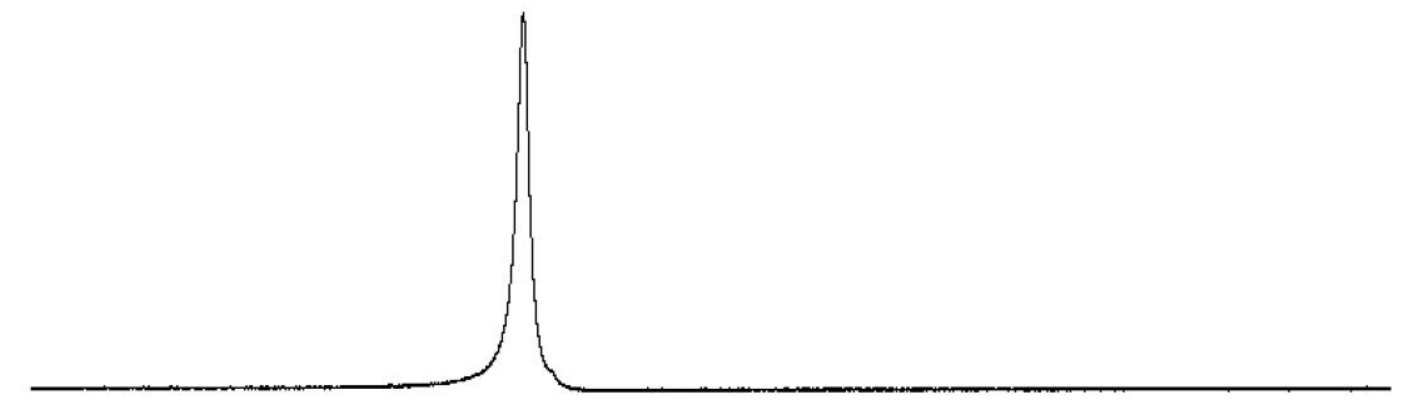

[ $\begin{array}{lllllllllllllllllllllllll}{ }_{120.0} & 110.0 & 100.0 & 90.0 & 80.0 & 70.0 & 60.0 & 50.0 & 40.0 & 30.0 & 20.0 & 10.0 & 0.0 & -10.0 & -20.0 & -30.0 & -40.0 & -50.0 & -60.0 & -70.0 & -80.0 & -90.0 & -100.0 & -110.0 & -120.0\end{array}$ 
${ }^{1} \mathrm{H}$ NMR spectra of $4 \mathrm{ga}\left(\mathrm{C}_{6} \mathrm{D}_{6}, 400 \mathrm{MHz}\right)$

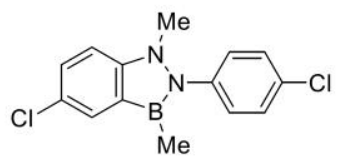

4ga
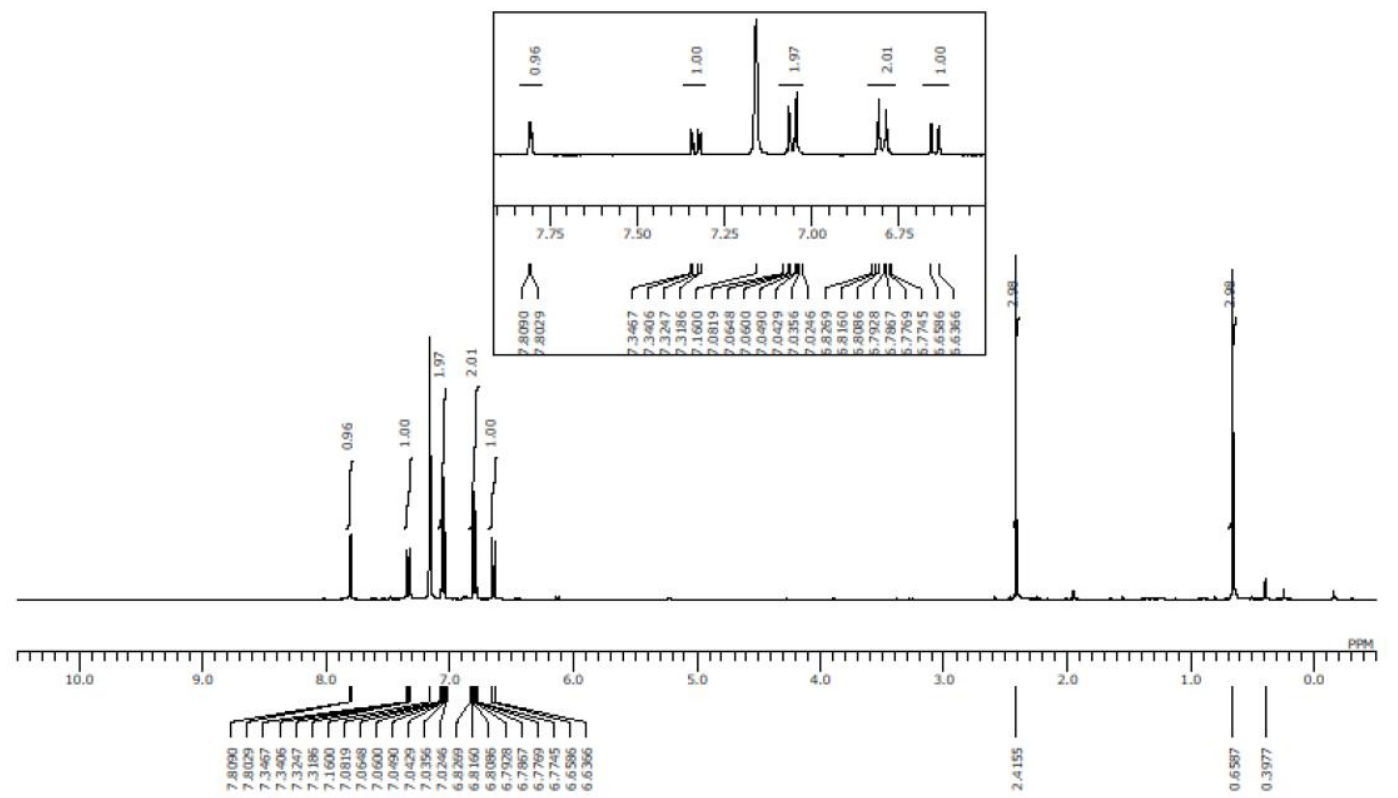

${ }^{13} \mathrm{C}$ NMR spectra of 4 ga $\left(\mathrm{CDCl}_{3}, 100 \mathrm{MHz}\right)$

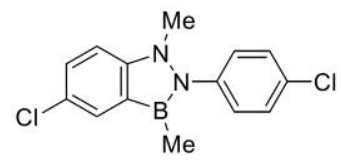

4ga
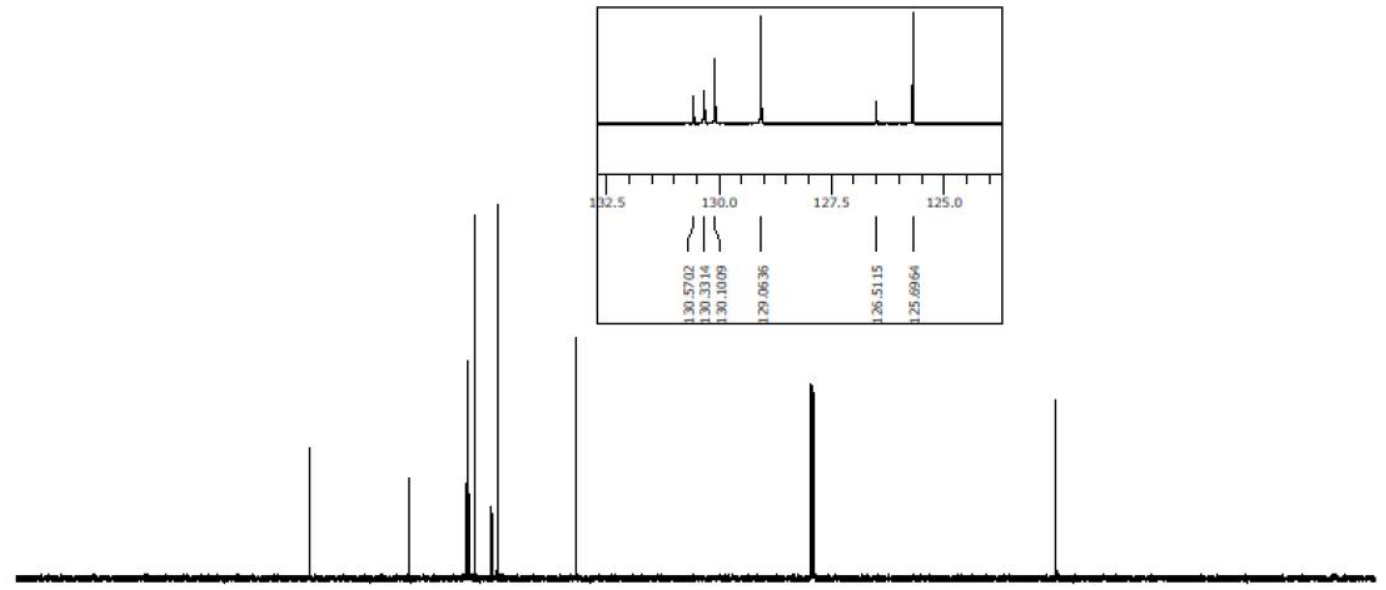

(

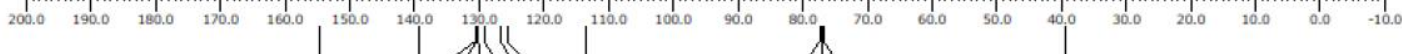

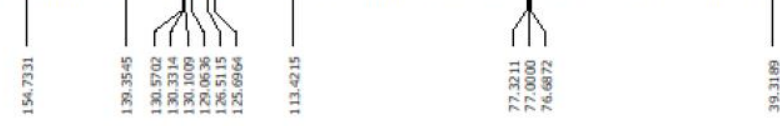


${ }^{11} \mathrm{~B}$ NMR spectra of $4 \mathrm{ga}\left(\mathrm{CDCl}_{3}, 193 \mathrm{MHz}\right)$

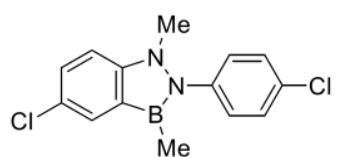

4ga

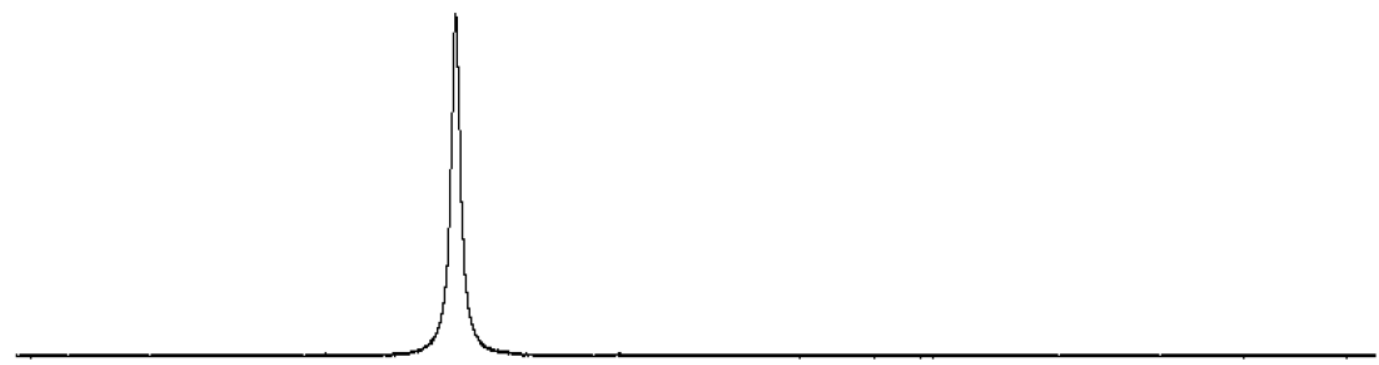

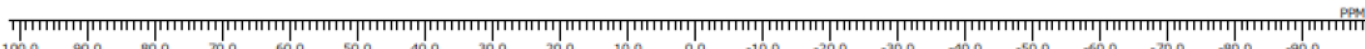

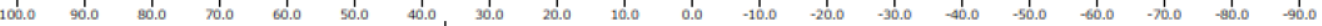
1 西

${ }^{1} \mathrm{H}$ NMR spectra of $\mathbf{4 g c}\left(\mathrm{C}_{6} \mathrm{D}_{6}, 400 \mathrm{MHz}\right)$
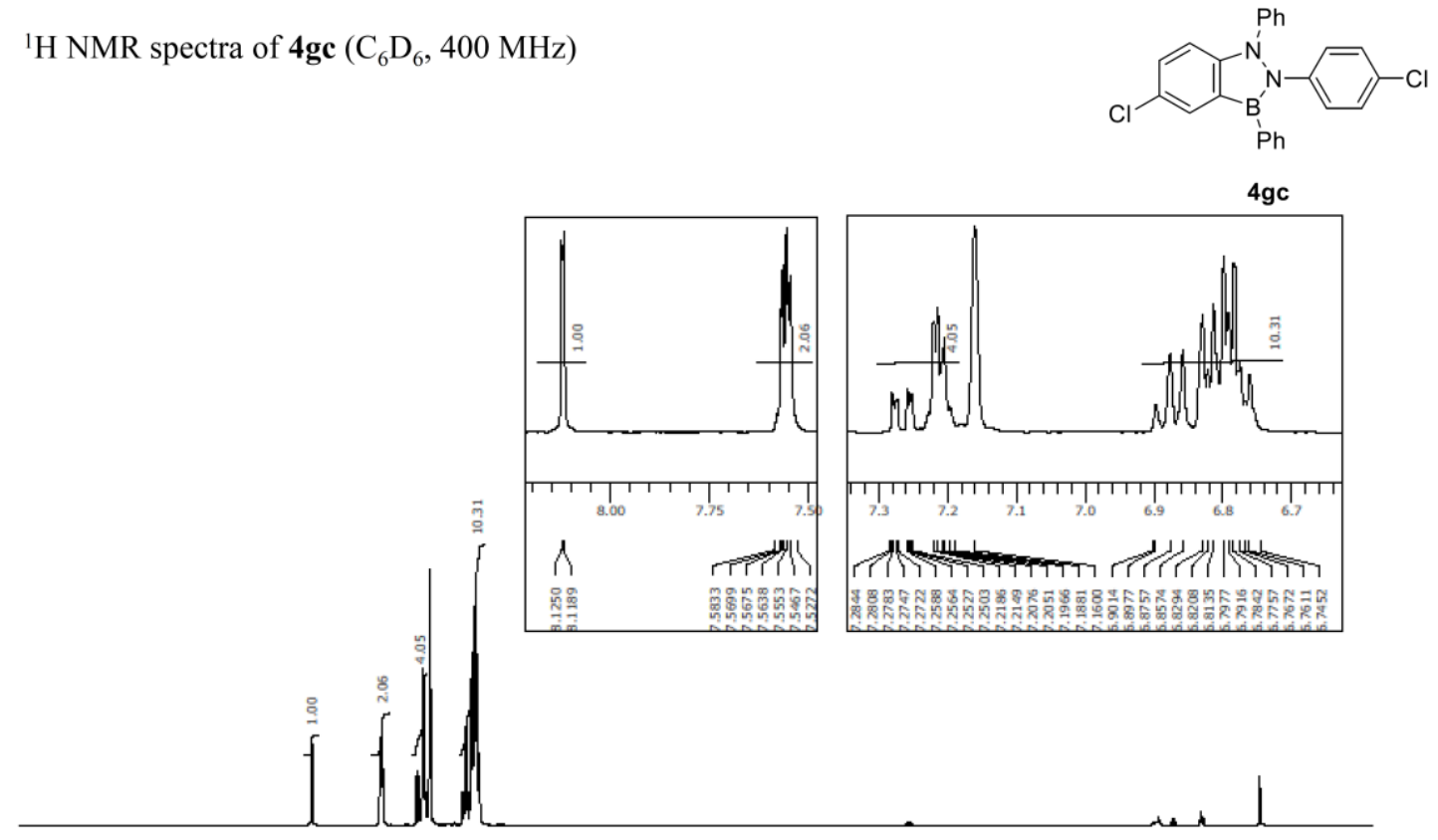

Г

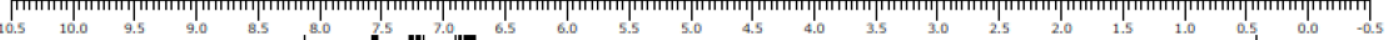

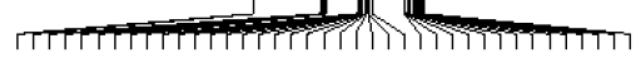

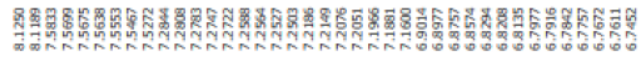


${ }^{13} \mathrm{C}$ NMR spectra of $\mathbf{4 g c}\left(\mathrm{CDCl}_{3}, 150 \mathrm{MHz}\right)$
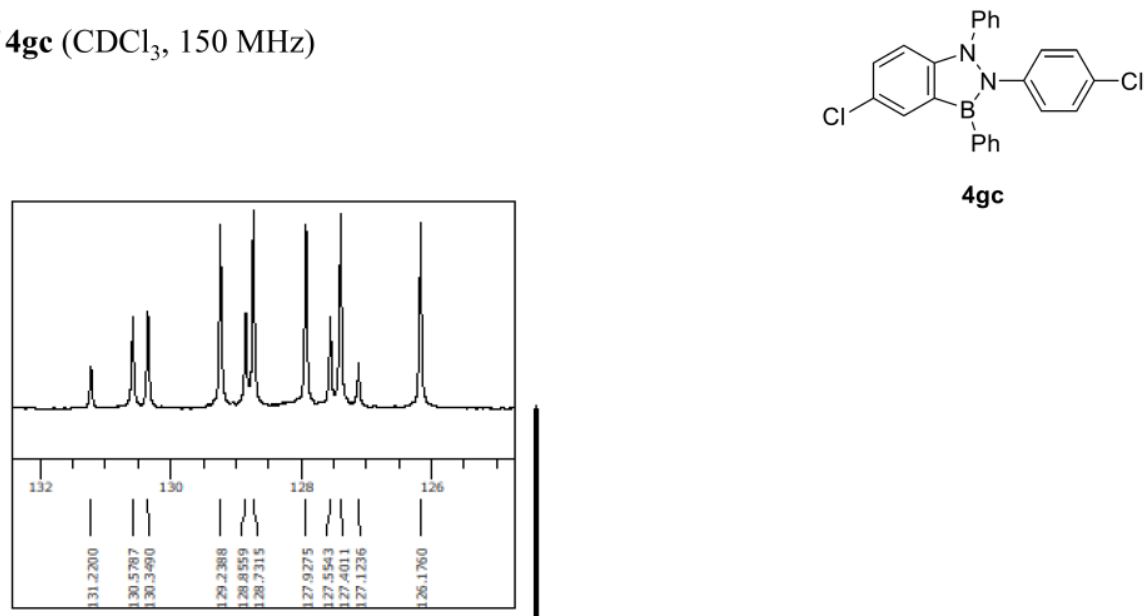

4gc

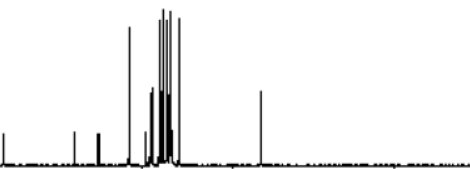

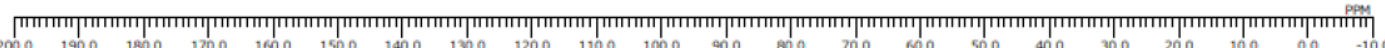

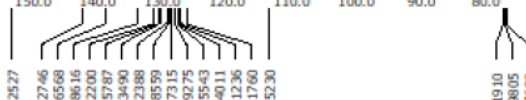

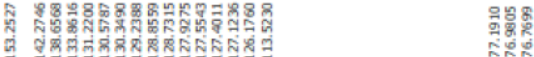

${ }^{11} \mathrm{~B}$ NMR spectra of $\mathbf{4 g c}\left(\mathrm{CDCl}_{3}, 193 \mathrm{MHz}\right)$

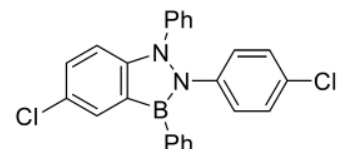

$4 \mathrm{gc}$

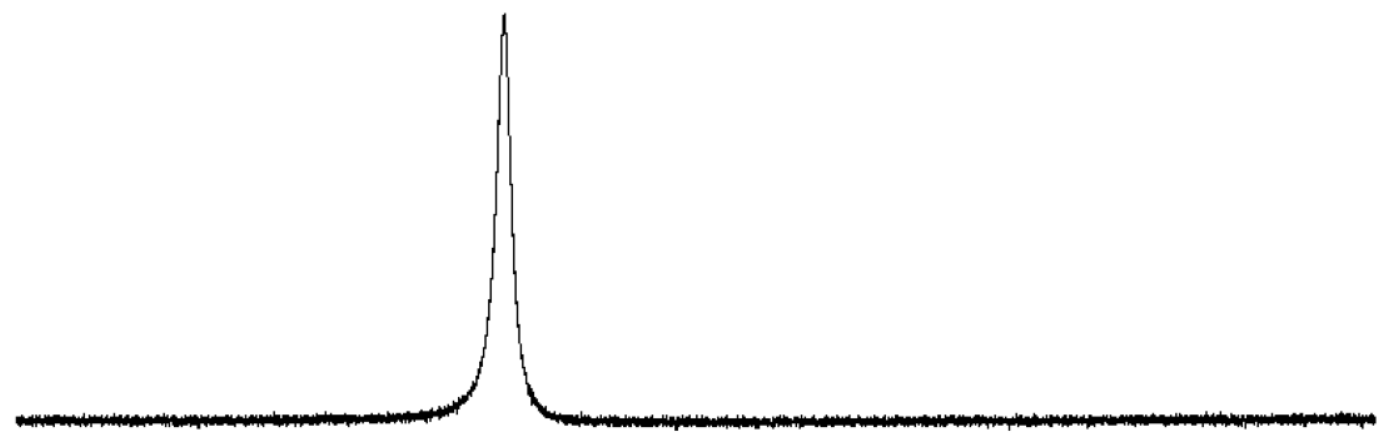

[

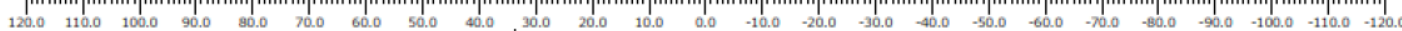


${ }^{1} \mathrm{H}$ NMR spectra of $\mathbf{4 h a}\left(\mathrm{CDCl}_{3}, 400 \mathrm{MHz}\right)$
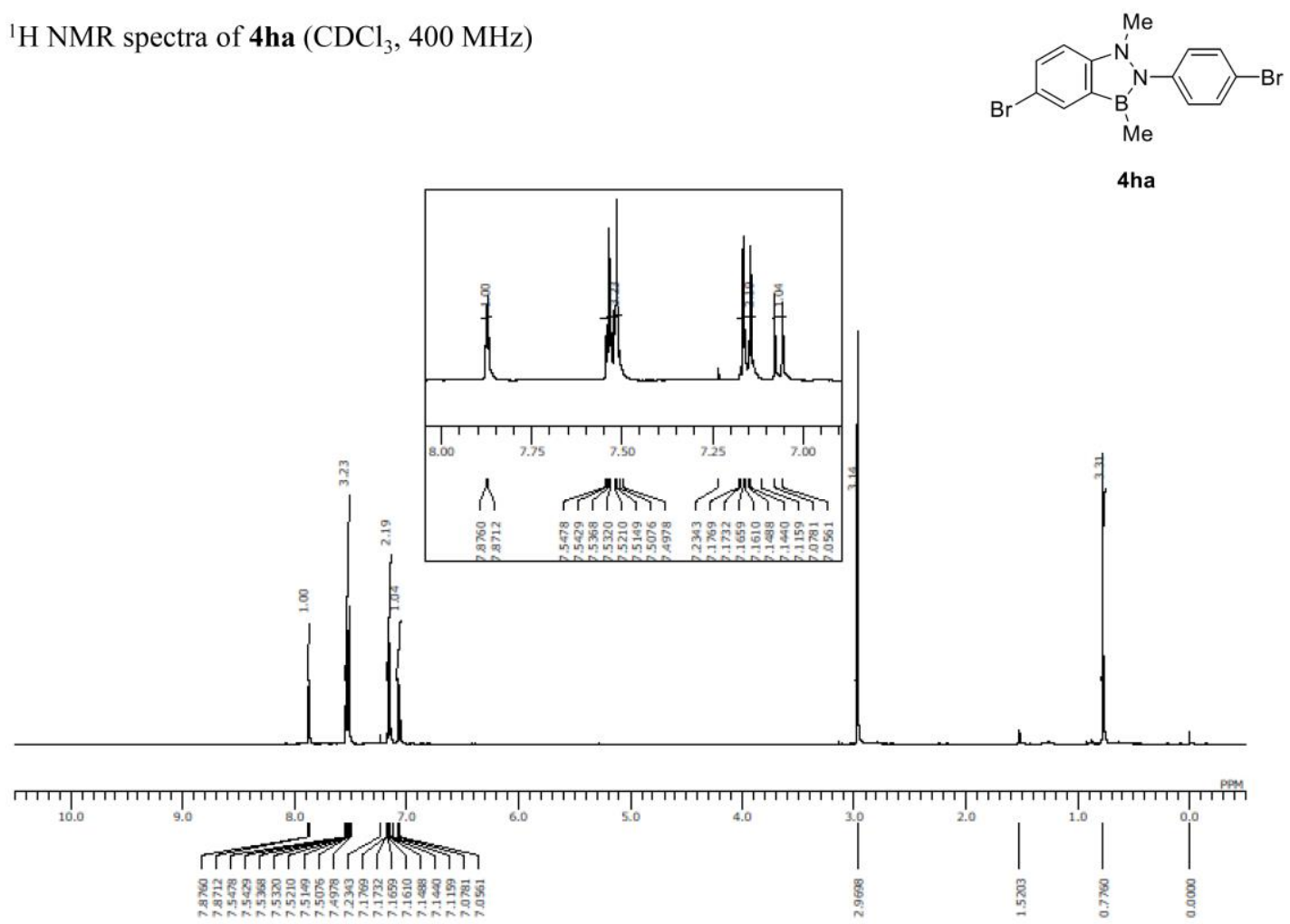

${ }^{13} \mathrm{C}$ NMR spectra of 4 ha $\left(\mathrm{CDCl}_{3}, 100 \mathrm{MHz}\right)$

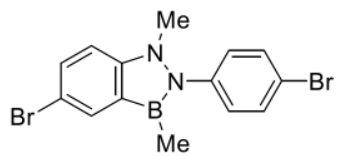

4ha

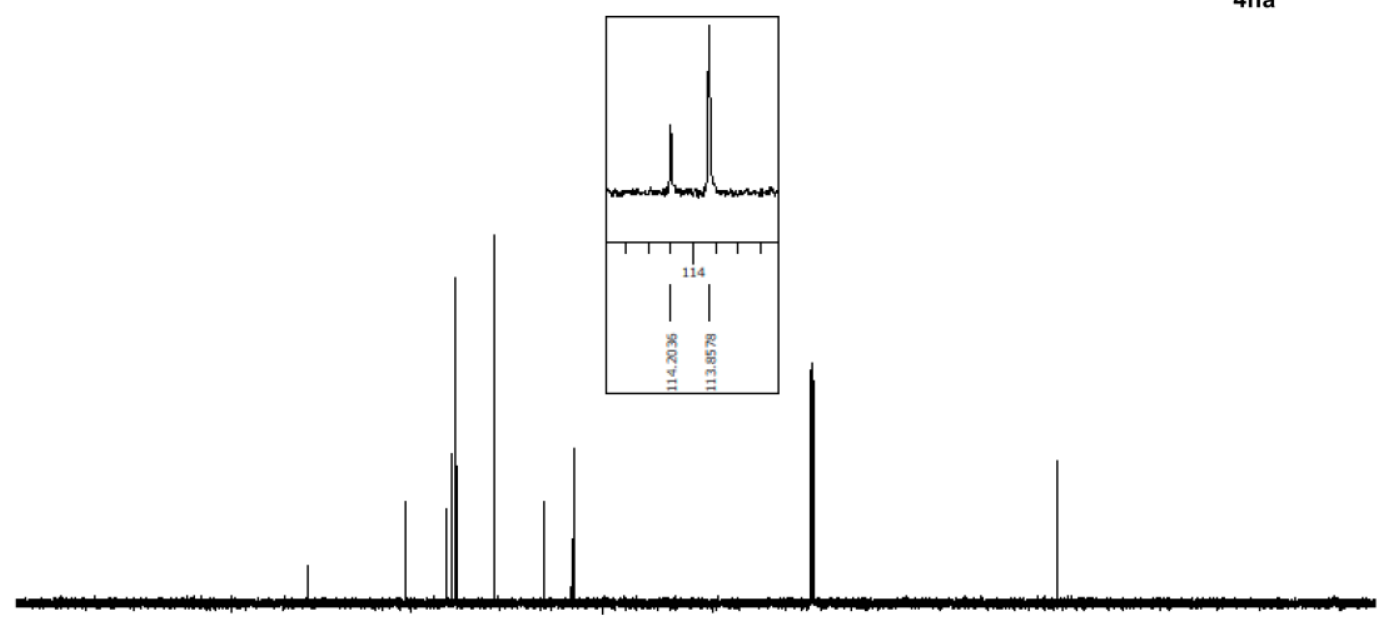

[

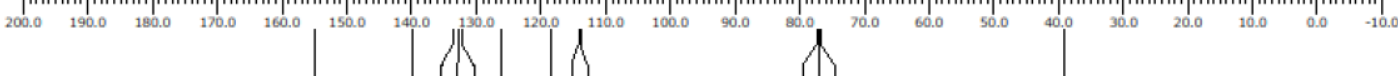

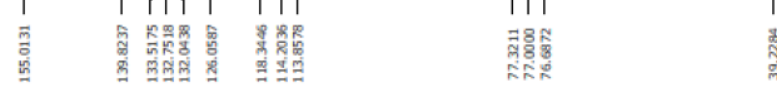


${ }^{11} \mathrm{~B}$ NMR spectra of $\mathbf{4 h a}\left(\mathrm{CDCl}_{3}, 193 \mathrm{MHz}\right)$

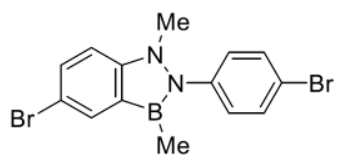

4ha

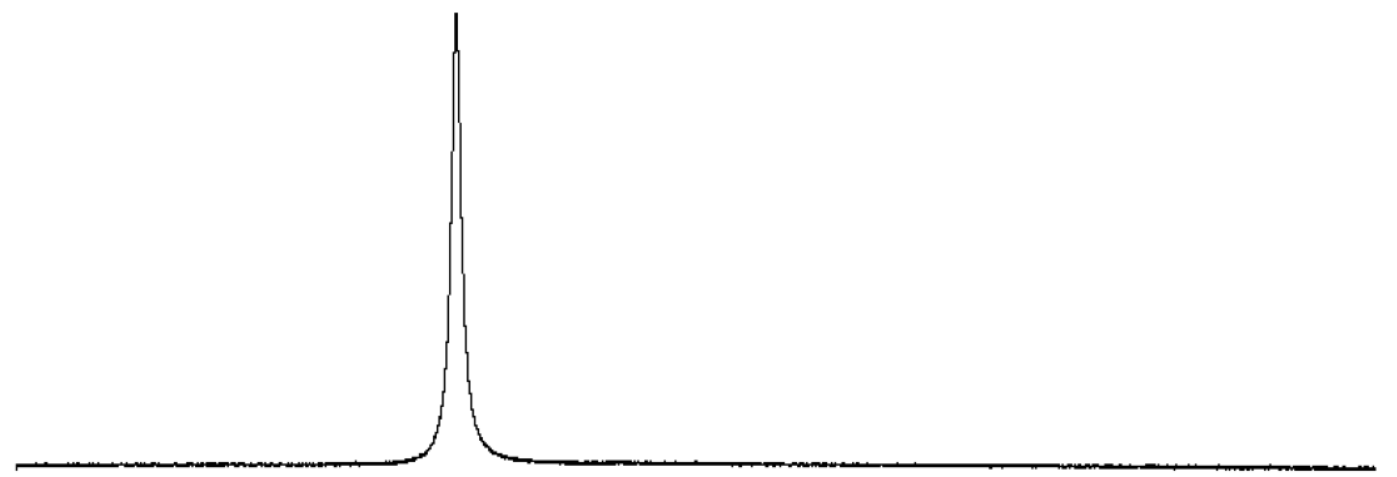

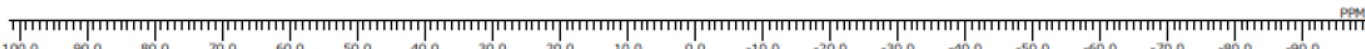

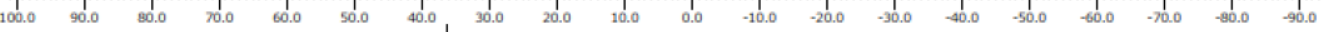

${ }^{1} \mathrm{H}$ NMR spectra of $\mathbf{4 h c}\left(\mathrm{CDCl}_{3}, 400 \mathrm{MHz}\right)$
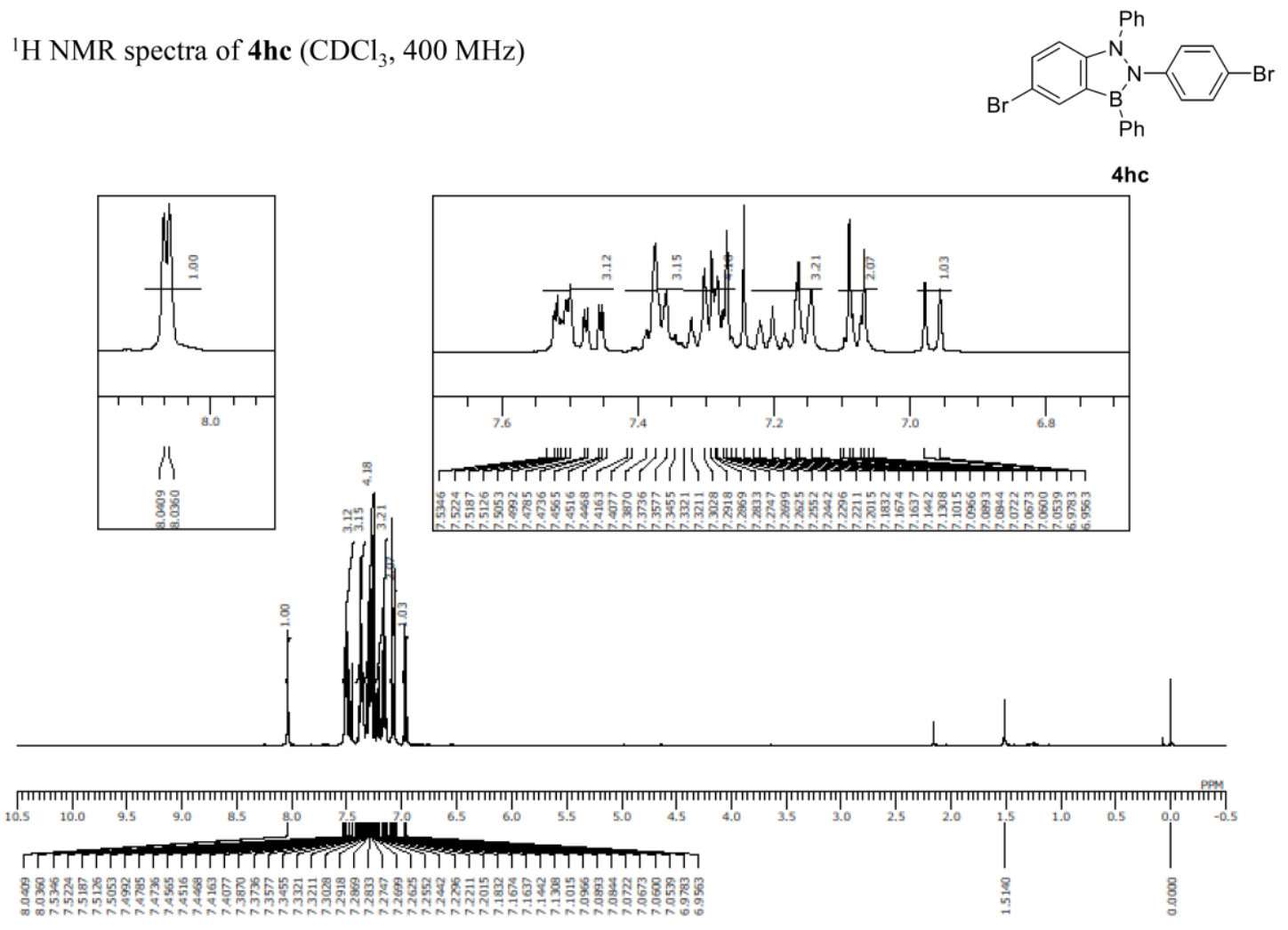
${ }^{13} \mathrm{C}$ NMR spectra of $\mathbf{4 h c}\left(\mathrm{CDCl}_{3}, 100 \mathrm{MHz}\right)$<smiles>Brc1ccc(-n2cc(-c3ccccc3)c3cc(Br)ccc32)cc1</smiles>

4hc
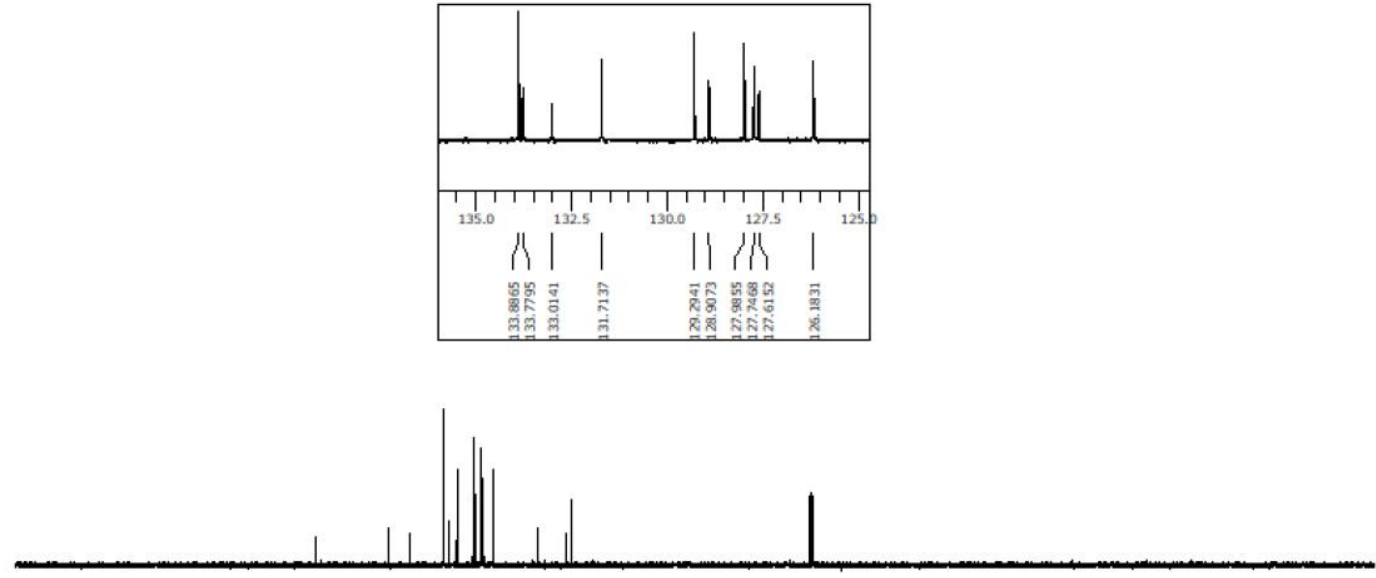

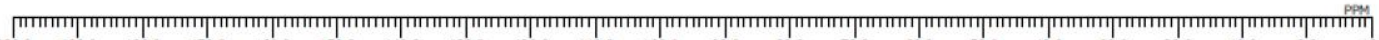

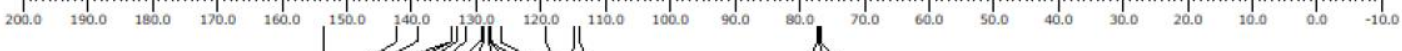
irßn

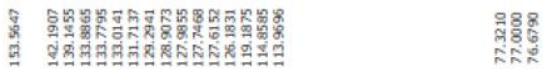

${ }^{11} \mathrm{~B}$ NMR spectra of $\mathbf{4 h c}\left(\mathrm{CDCl}_{3}, 193 \mathrm{MHz}\right)$

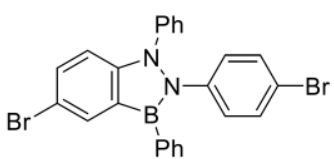

4hc

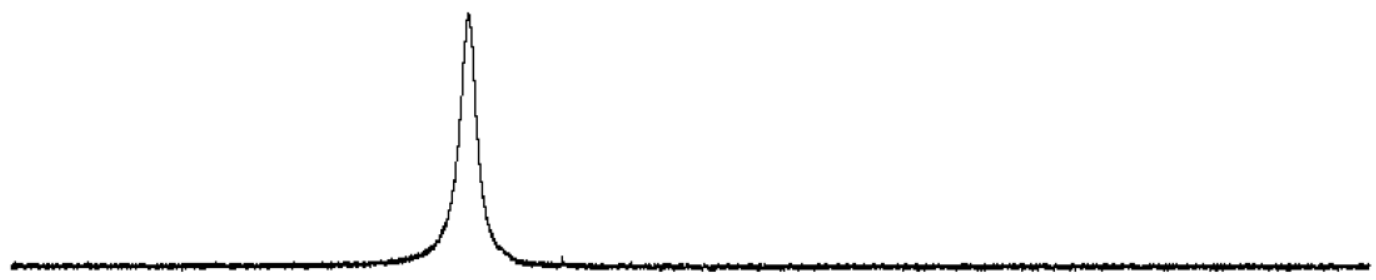

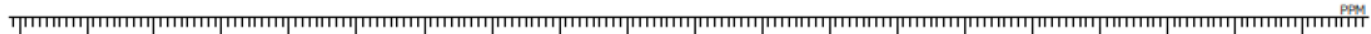

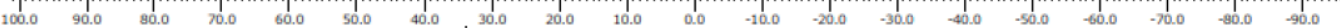


${ }^{1} \mathrm{H}$ NMR spectra of $4 \mathrm{ia}\left(\mathrm{C}_{6} \mathrm{D}_{6}, 400 \mathrm{MHz}\right)$
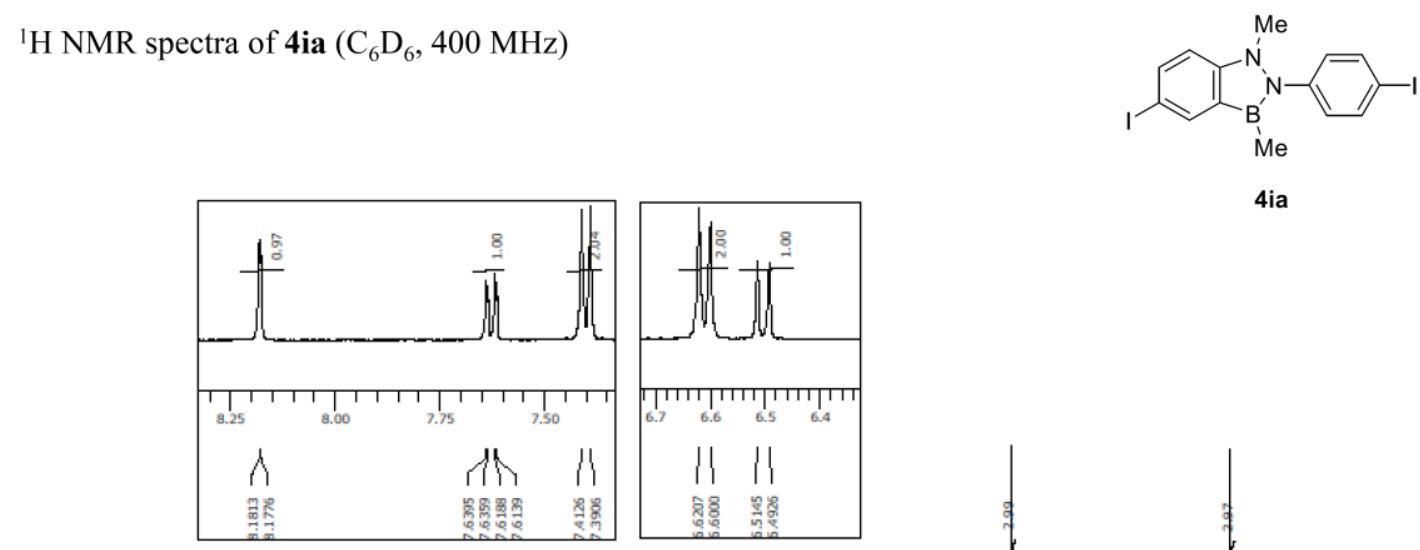

$4 i a$

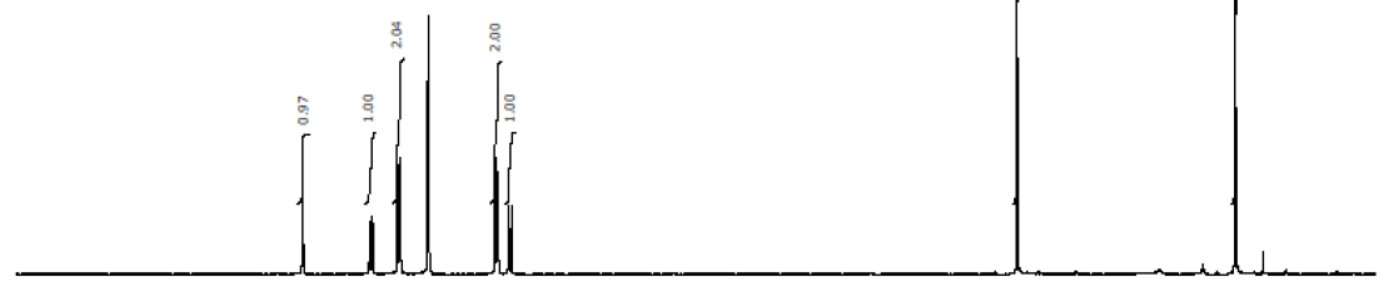

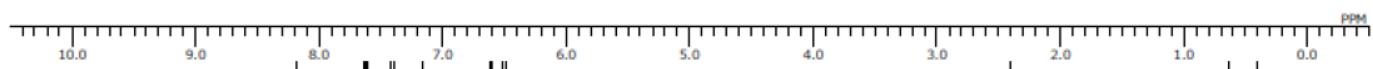
A

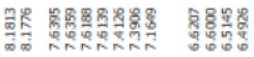

${ }^{13} \mathrm{C}$ NMR spectra of $4 \mathrm{ia}\left(\mathrm{CDCl}_{3}, 100 \mathrm{MHz}\right)$

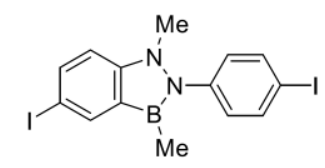

$4 i a$
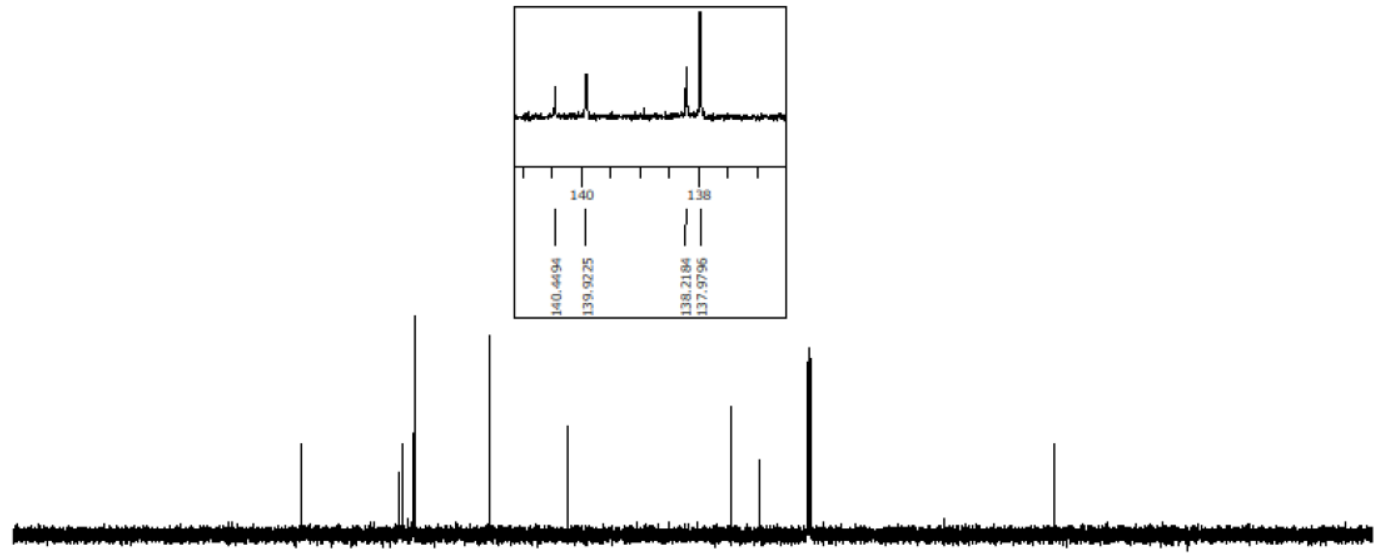

[

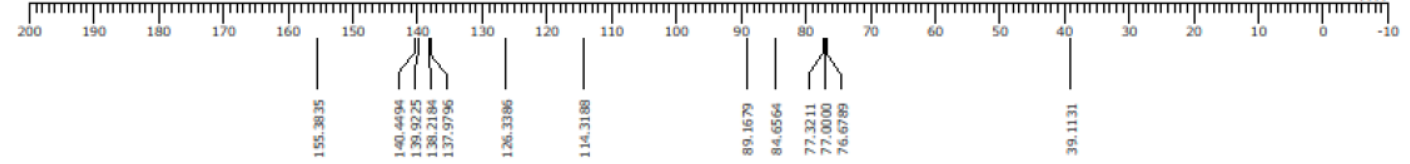


${ }^{11} \mathrm{~B}$ NMR spectra of $4 \mathrm{ia}\left(\mathrm{C}_{6} \mathrm{D}_{6}, 193 \mathrm{MHz}\right)$

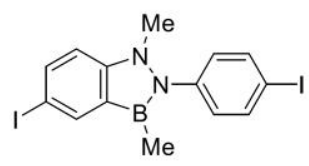

4 ia

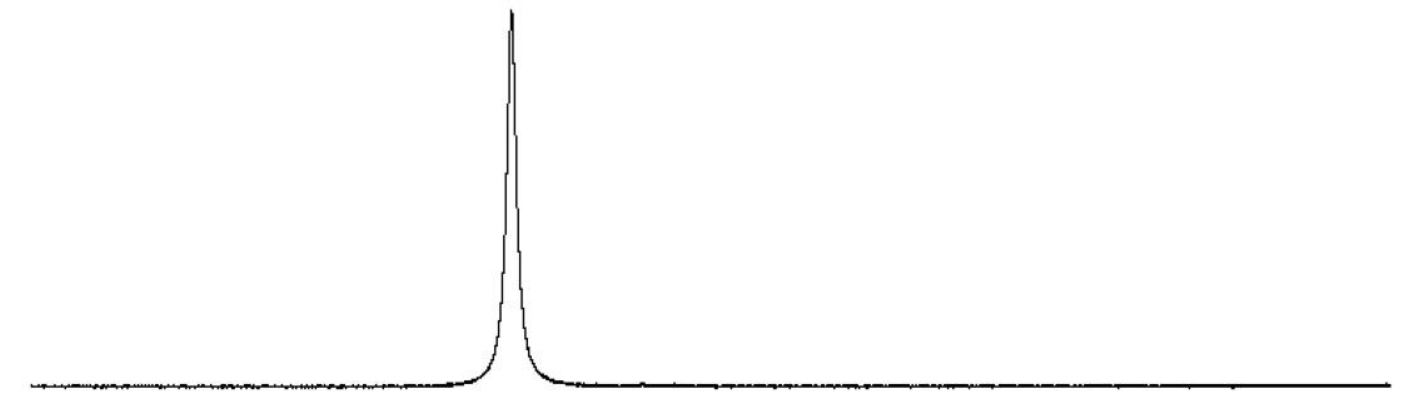

(

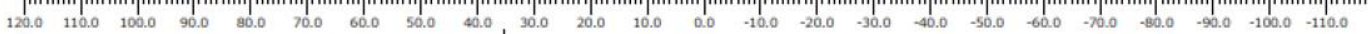

${ }^{1} \mathrm{H}$ NMR spectra of 4 ic $\left(\mathrm{C}_{6} \mathrm{D}_{6}, 400 \mathrm{MHz}\right)$
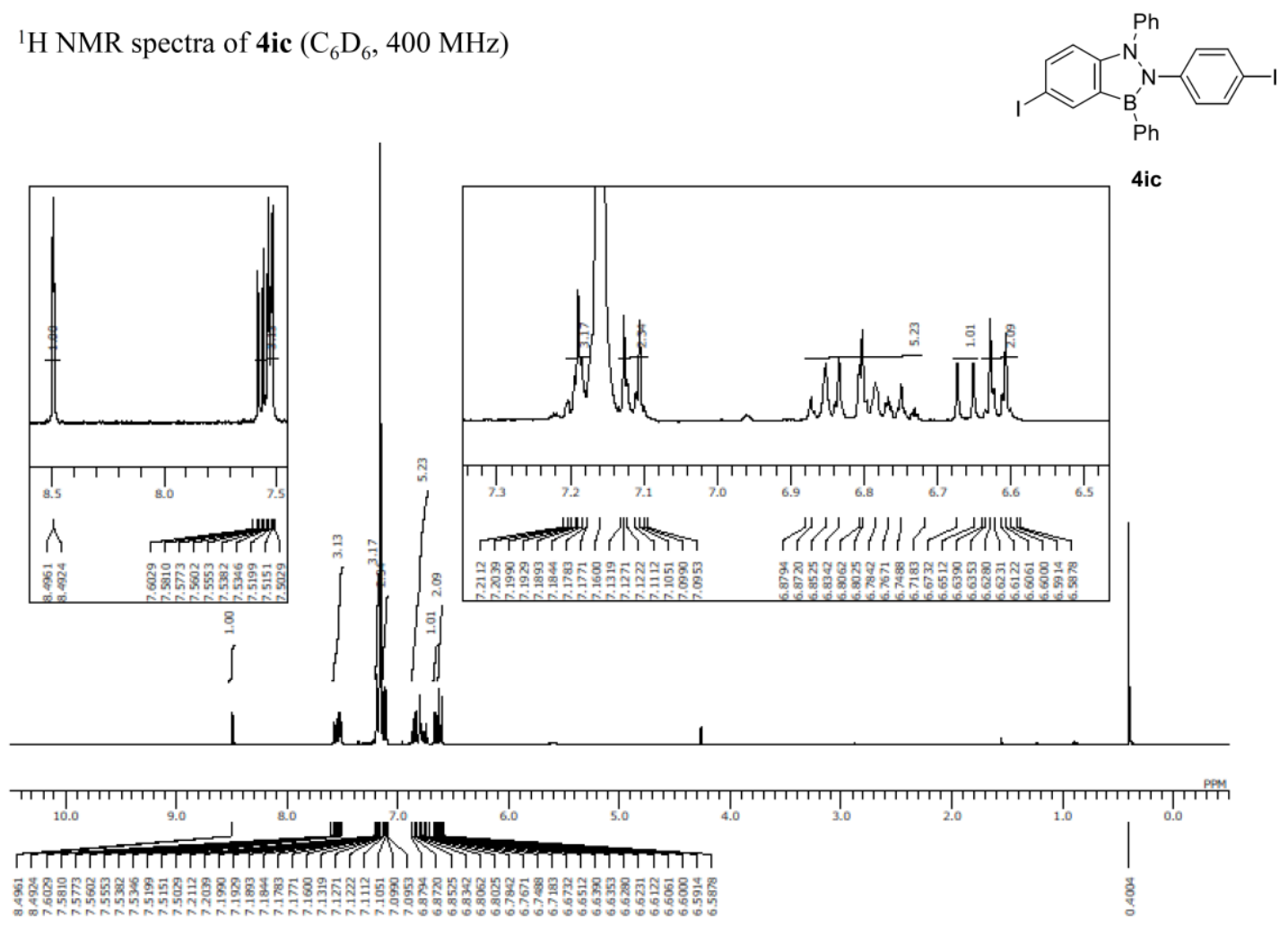
${ }^{13} \mathrm{C}$ NMR spectra of 4 ic $\left(\mathrm{CDCl}_{3}, 150 \mathrm{MHz}\right)$
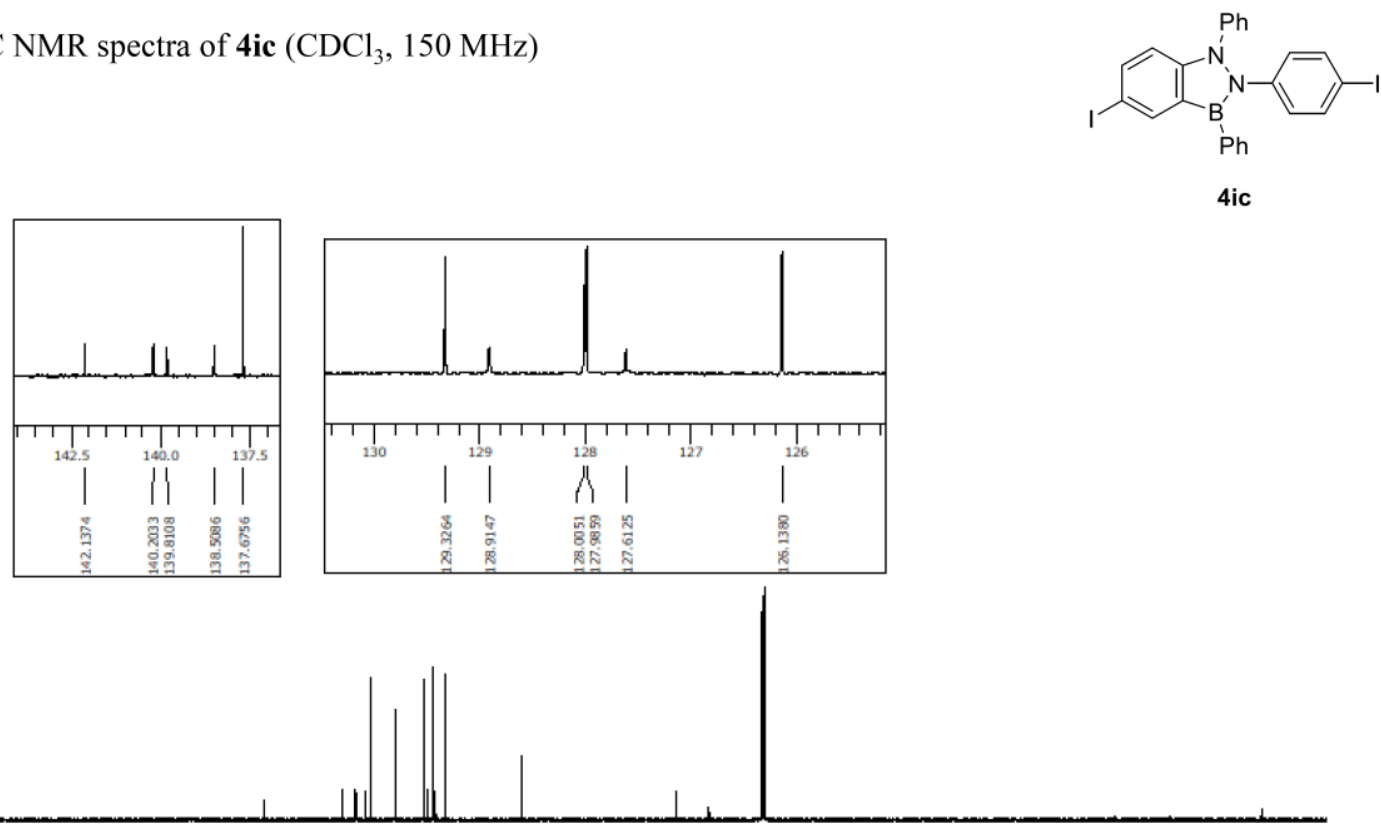

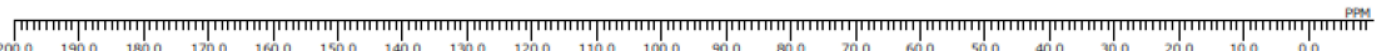

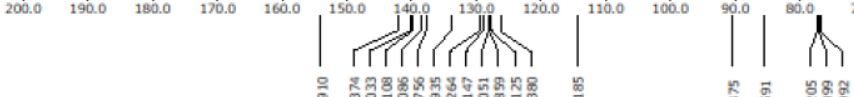

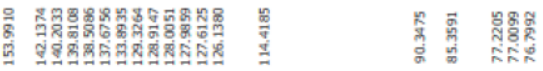

${ }^{11} \mathrm{~B}$ NMR spectra of $4 \mathrm{ic}\left(\mathrm{CDCl}_{3}, 193 \mathrm{MHz}\right)$

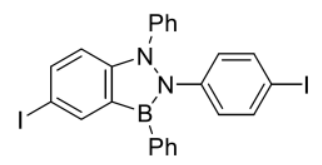

4ic

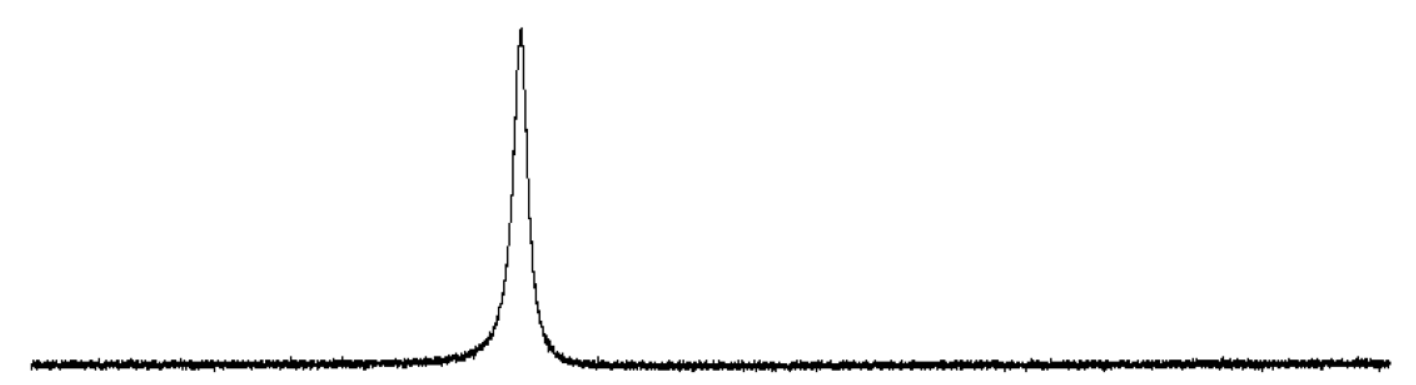

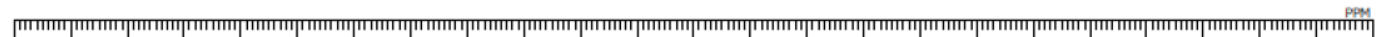

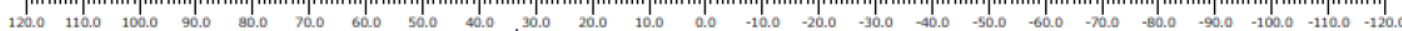


${ }^{1} \mathrm{H}$ NMR spectra of $\mathbf{4 j a}\left(\mathrm{CDCl}_{3}, 400 \mathrm{MHz}\right)$
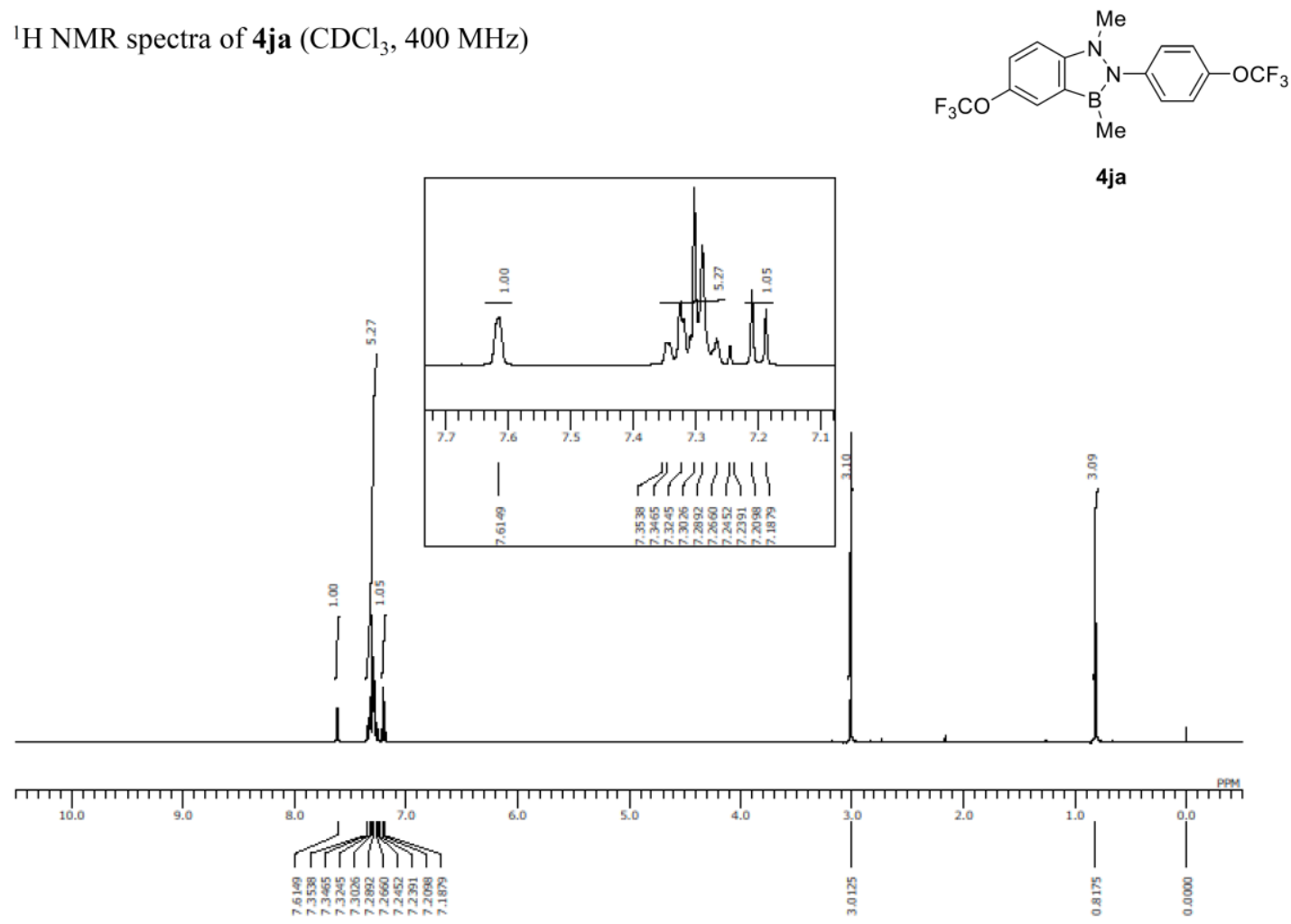

${ }^{13} \mathrm{C}$ NMR spectra of $\mathbf{4 j a}\left(\mathrm{CDCl}_{3}, 100 \mathrm{MHz}\right)$

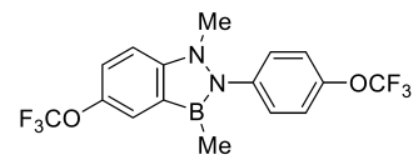

4ja
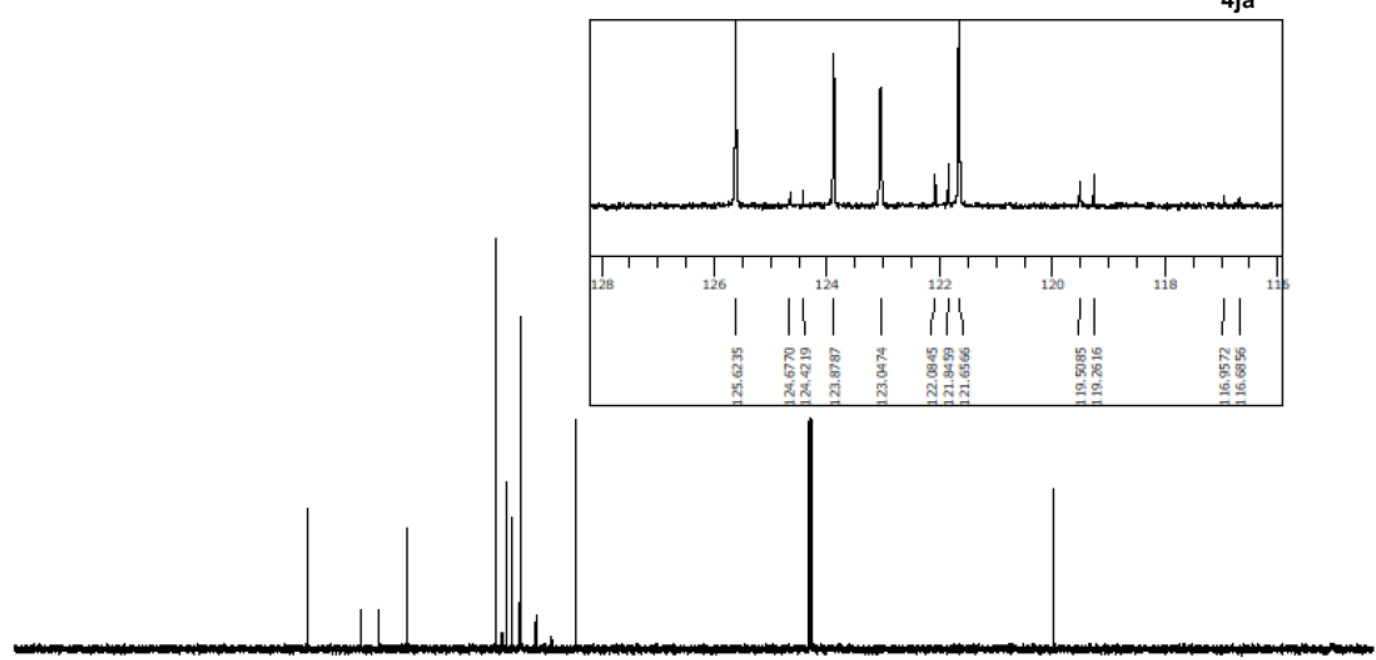

(

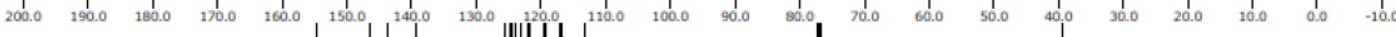

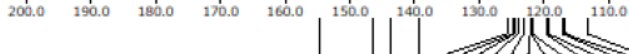

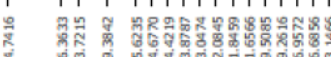

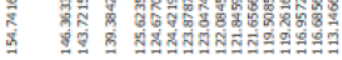


${ }^{11} \mathrm{~B}$ NMR spectra of $\mathbf{4 j a}\left(\mathrm{CDCl}_{3}, 193 \mathrm{MHz}\right)$
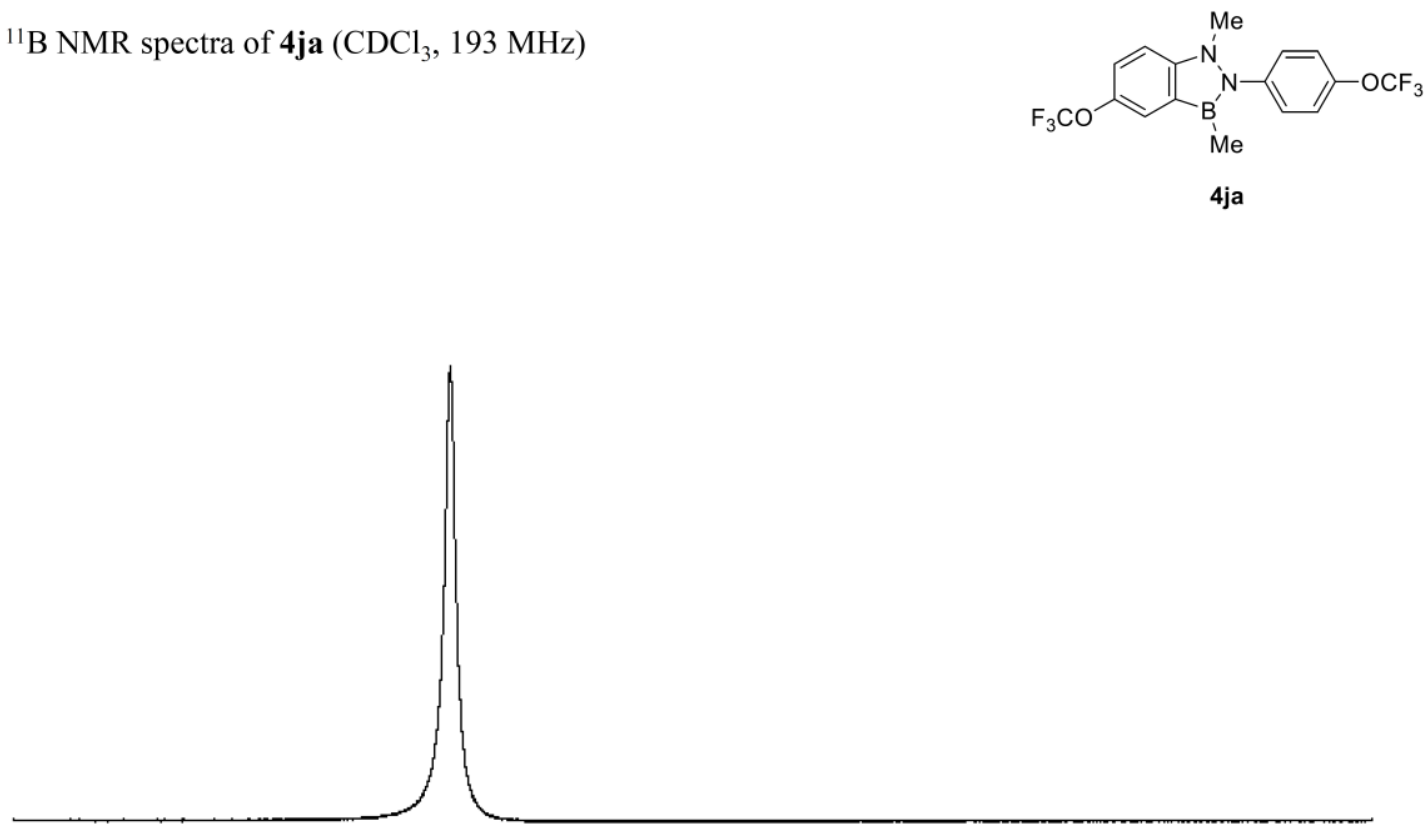

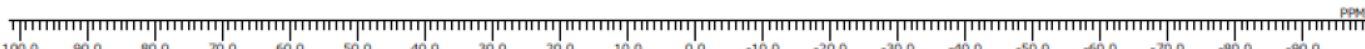

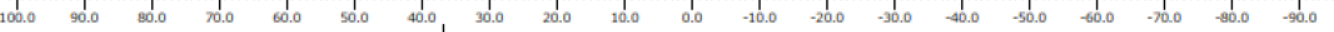

${ }^{1} \mathrm{H}$ NMR spectra of $\mathbf{4 j c}\left(\mathrm{C}_{6} \mathrm{D}_{6}, 400 \mathrm{MHz}\right)$

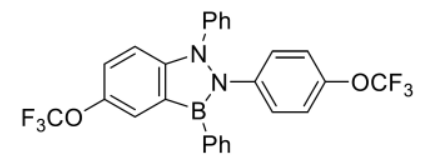

$4 \mathrm{jc}$

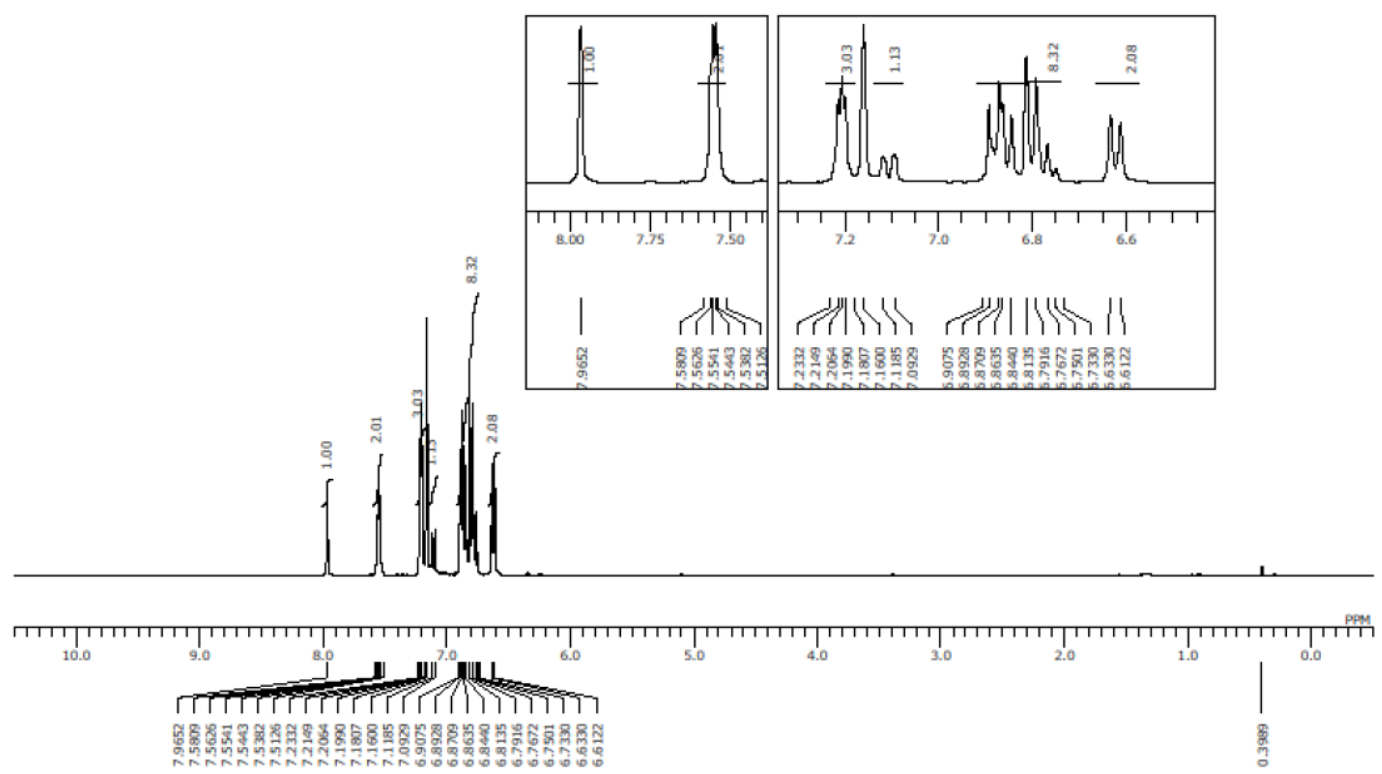


${ }^{13} \mathrm{C}$ NMR spectra of $\mathbf{4 j c}\left(\mathrm{CDCl}_{3}, 100 \mathrm{MHz}\right)$

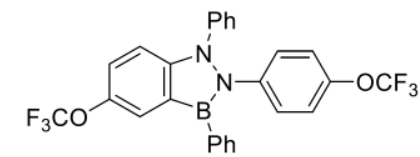

$4 \mathrm{jc}$
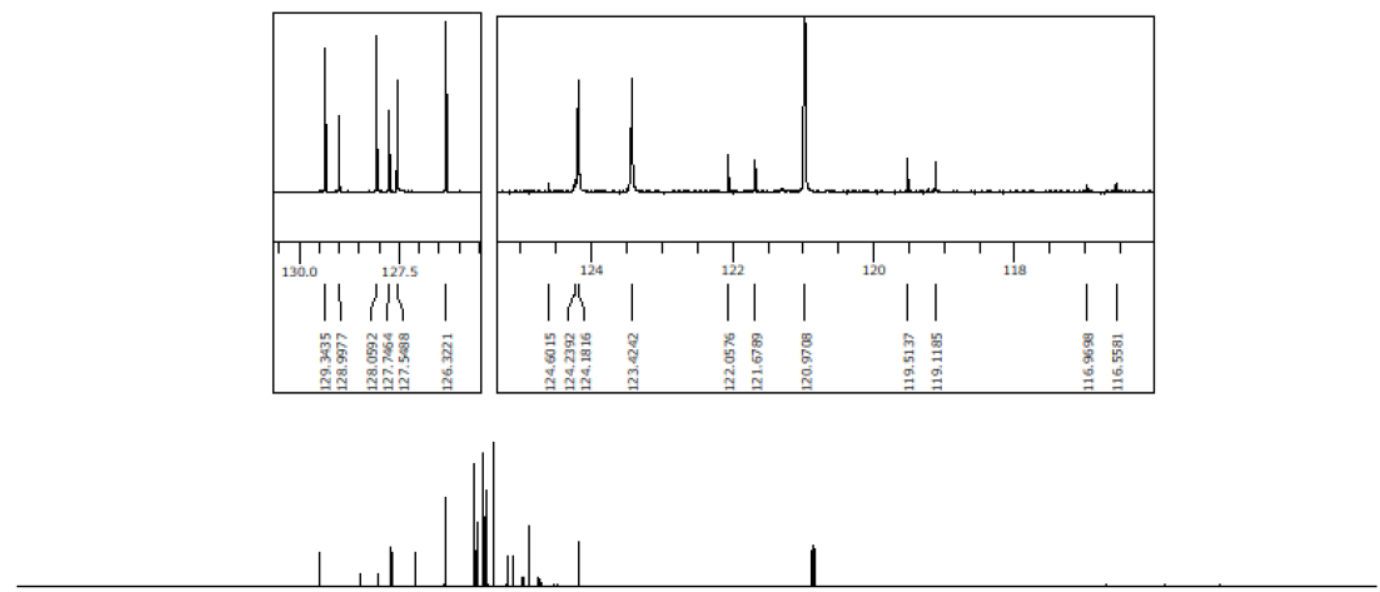

Г

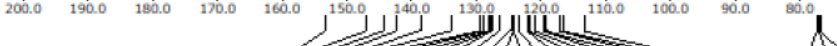

एाता

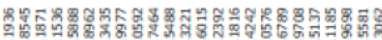

M)

츄용

${ }^{11} \mathrm{~B}$ NMR spectra of $\mathbf{4 j c}\left(\mathrm{C}_{6} \mathrm{D}_{6}, 193 \mathrm{MHz}\right)$

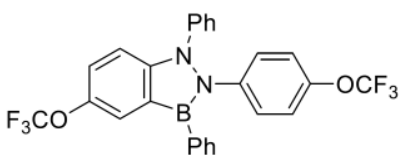

$4 \mathrm{jc}$

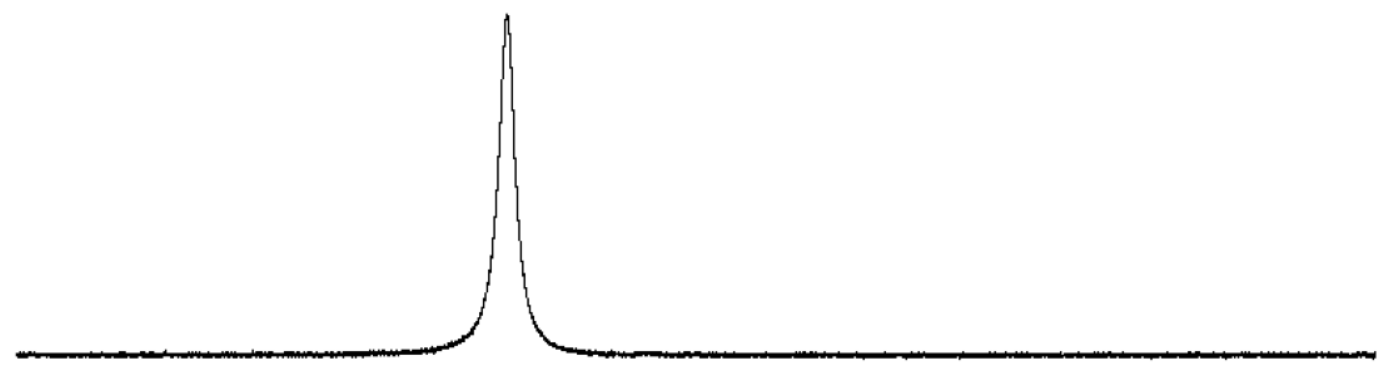

( $\begin{array}{llllllllllllllllllllllllll}120.0 & 110.0 & 100.0 & 90.0 & 90.0 & 70.0 & 60.0 & 50.0 & 40.0 & 30.0 & 20.0 & 10.0 & 0.0 & -10.0 & -20.0 & -30.0 & -40.0 & -50.0 & -60.0 & -70.0 & -80.0 & -90.0 & -100.0 & -110.0 & -120.0\end{array}$ 
${ }^{1} \mathrm{H}$ NMR spectra of $\mathbf{5}\left(\mathrm{C}_{6} \mathrm{D}_{6}, 400 \mathrm{MHz}\right)$

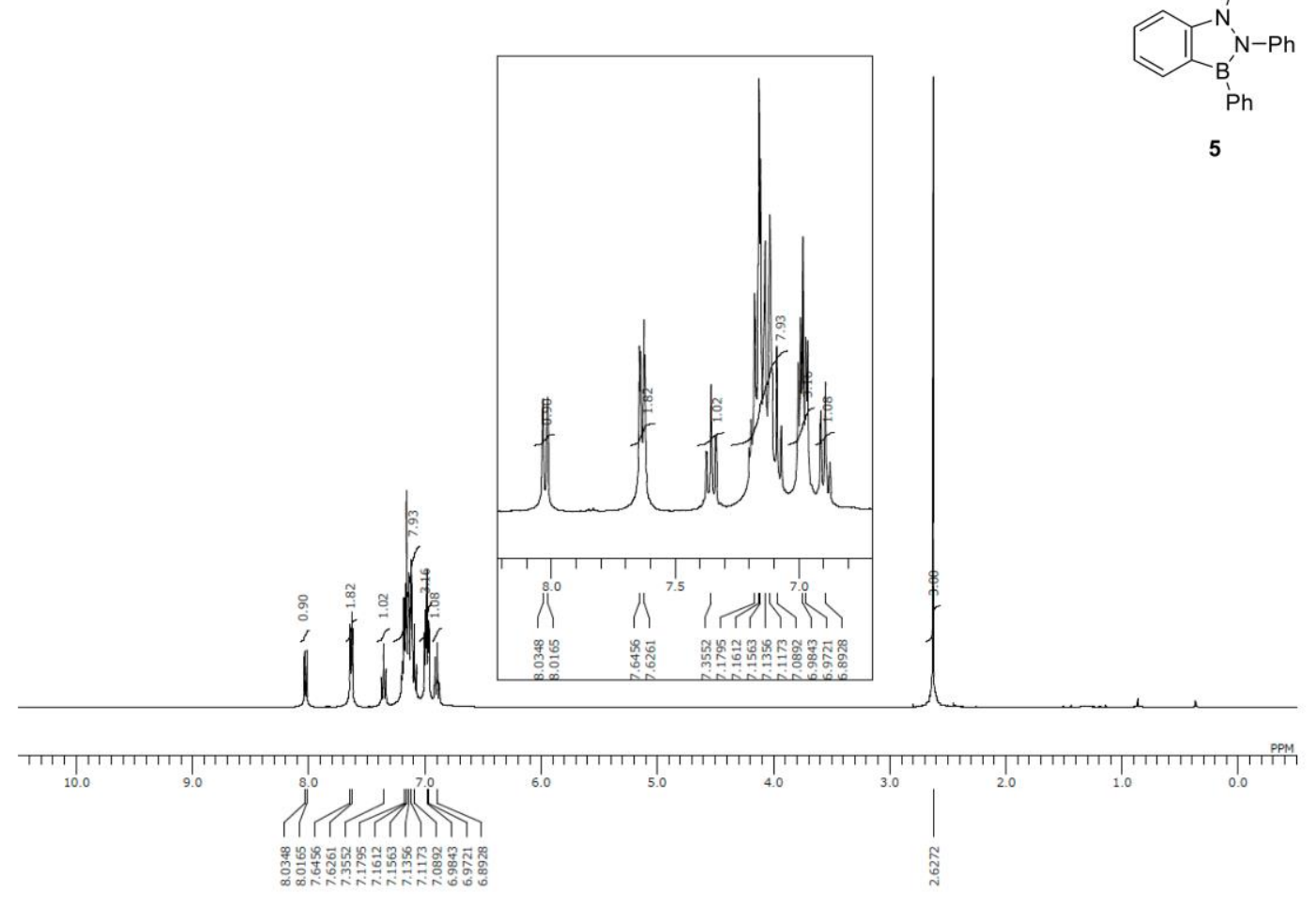

${ }^{13} \mathrm{C}$ NMR spectra of $5\left(\mathrm{C}_{6} \mathrm{D}_{6}, 150 \mathrm{MHz}\right)$

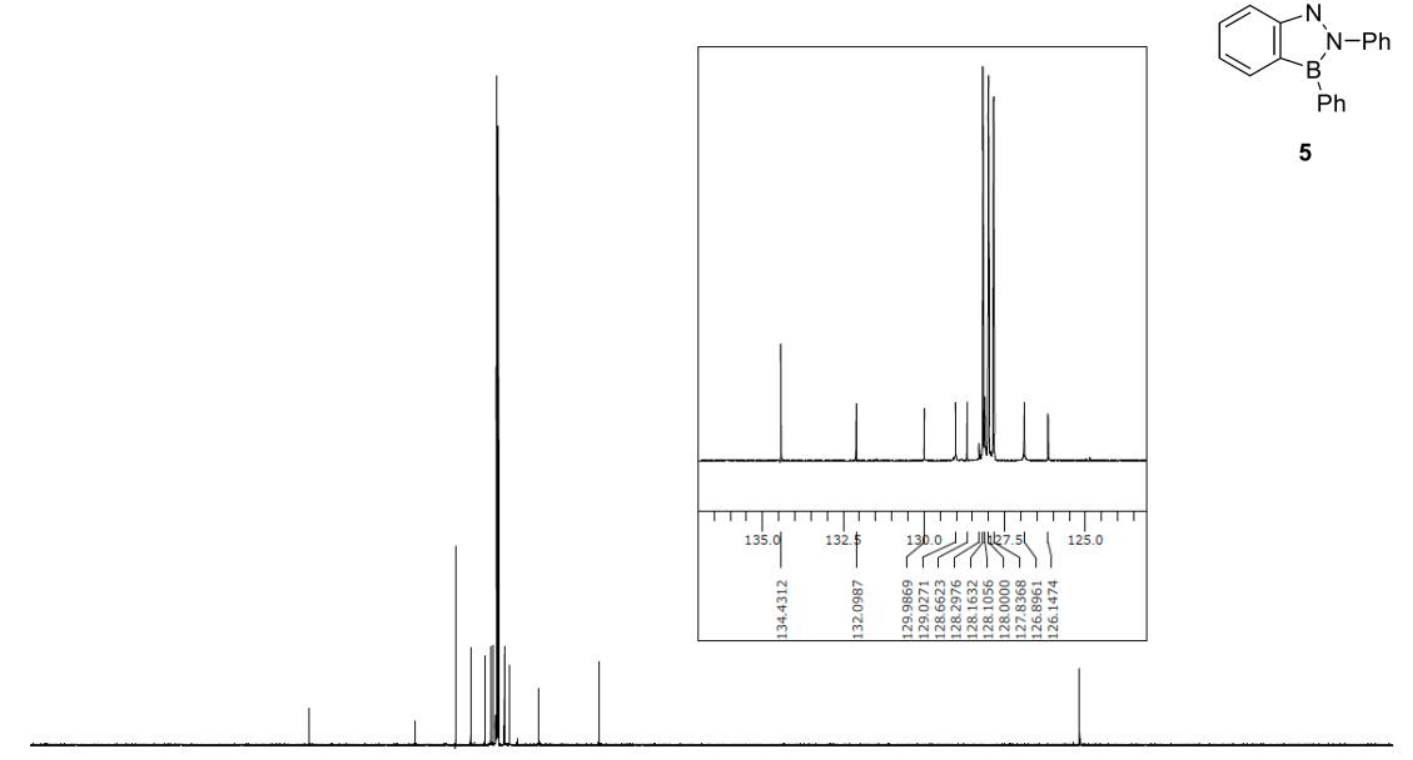

(1)

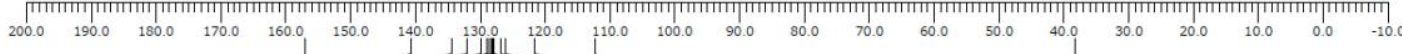

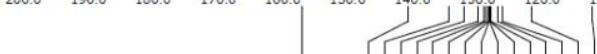

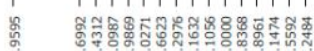

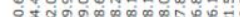

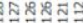


${ }^{11} \mathrm{~B}$ NMR spectra of $5\left(\mathrm{C}_{6} \mathrm{D}_{6}, 193 \mathrm{MHz}\right)$
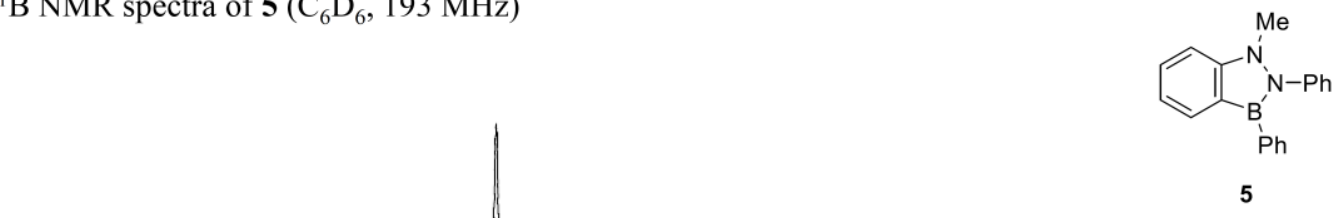

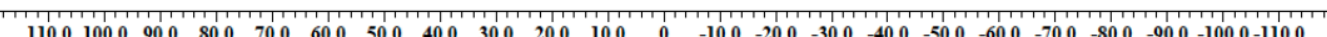

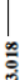

${ }^{1} \mathrm{H}$ NMR spectra of $6\left(\mathrm{C}_{6} \mathrm{D}_{6}, 400 \mathrm{MHz}\right)$
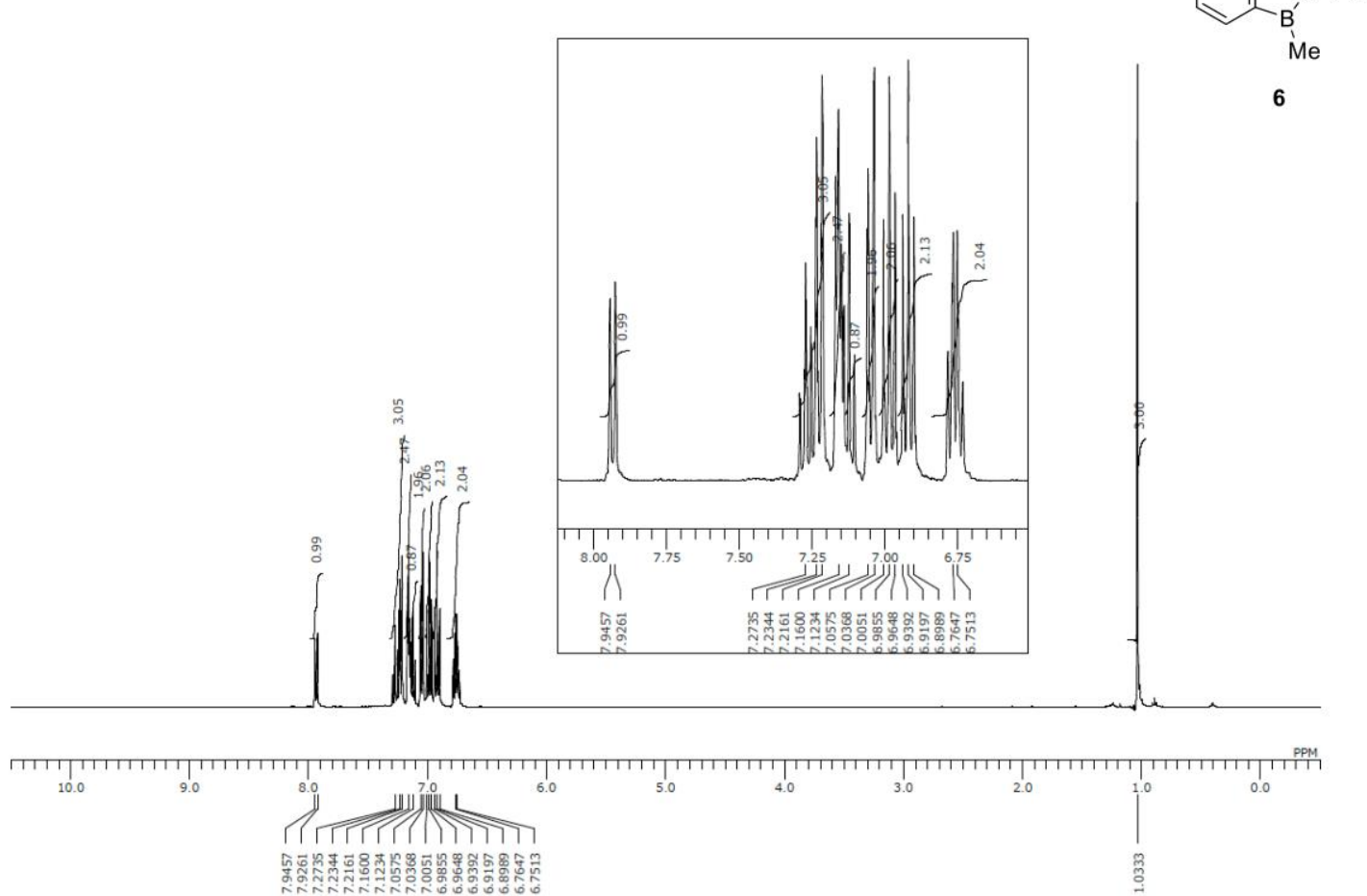
${ }^{13} \mathrm{C}$ NMR spectra of $6\left(\mathrm{C}_{6} \mathrm{D}_{6}, 150 \mathrm{MHz}\right)$
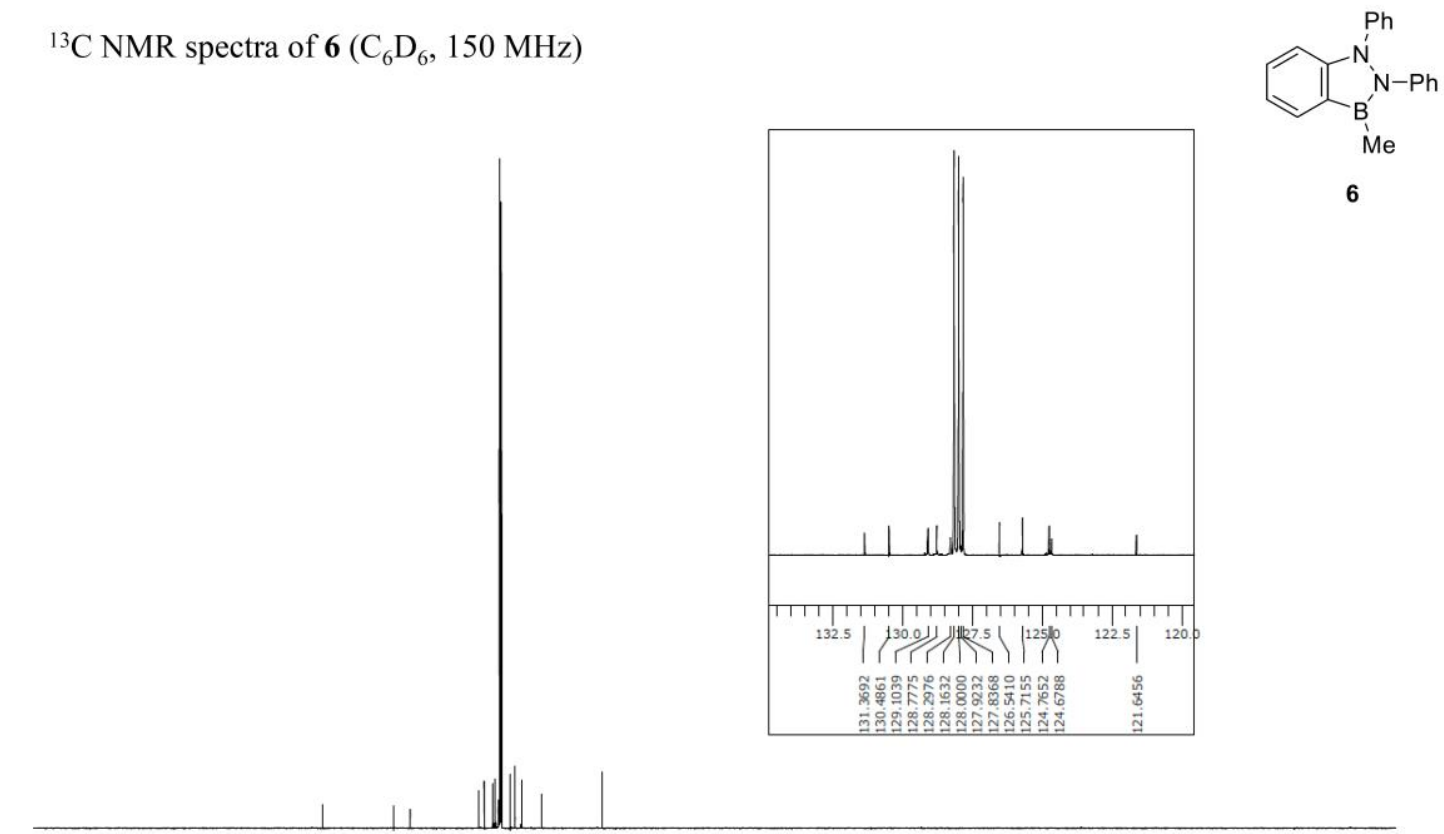

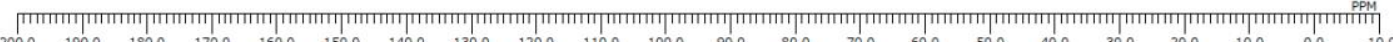
$\begin{array}{llllllllllllllllllllll}200.0 & 190.0 & 180.0 & 170.0 & 160.0 & 150.0 & 140.0 & 130.0 & 120.0 & 110.0 & 100.0 & 90.0 & 80.0 & 70.0 & 60.0 & 50.0 & 40.0 & 30.0 & 20.0 & 100 & 0.0 & -1\end{array}$ $1 / 1 / 117$

\&

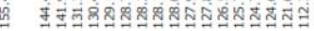

${ }^{11} \mathrm{~B}$ NMR spectra of $6\left(\mathrm{C}_{6} \mathrm{D}_{6}, 193 \mathrm{MHz}\right)$
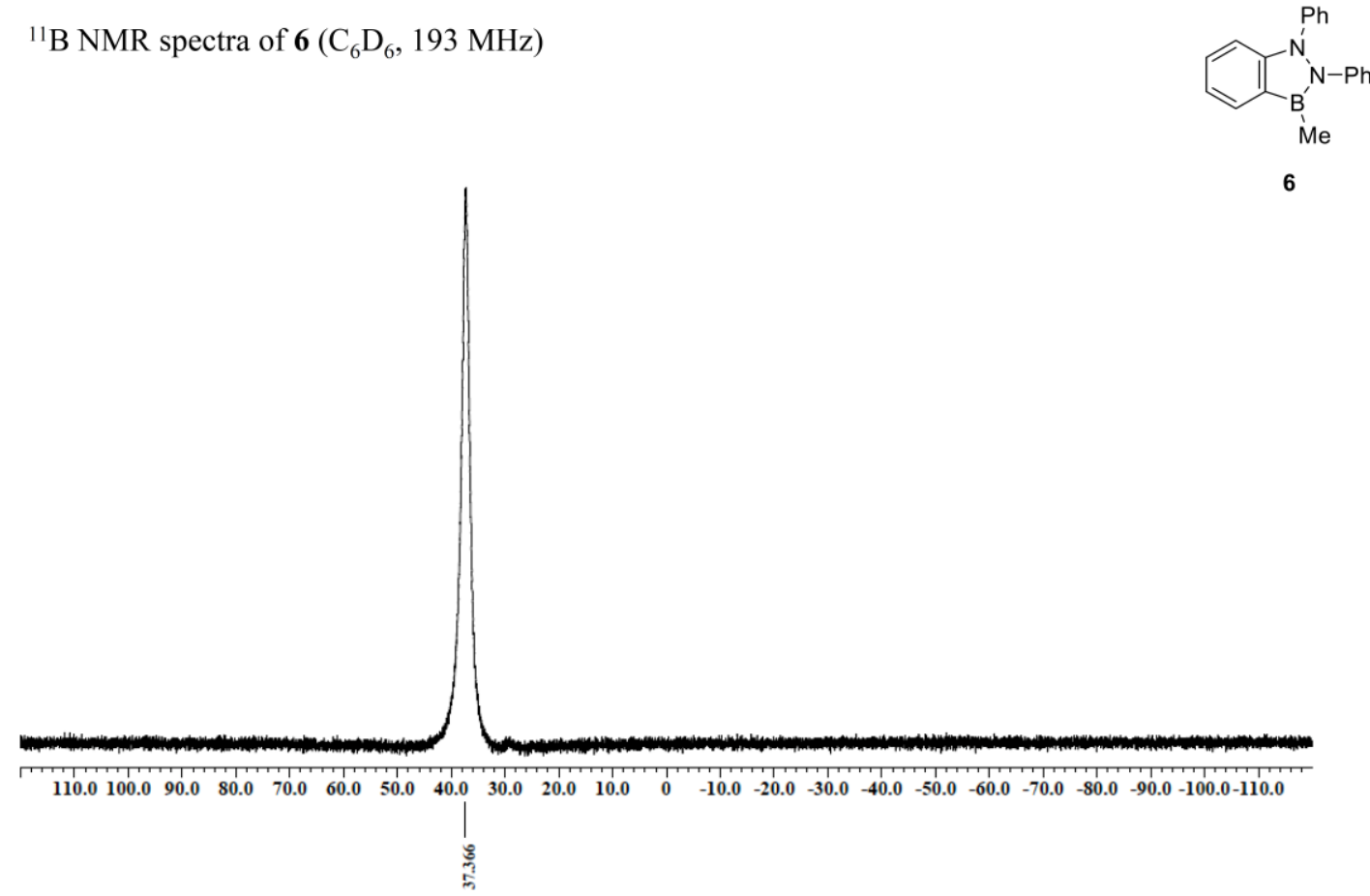